STRUCTURAL AND TRANSPORT PROPERTIES OF EPITAXIAL NIOBIUM-DOPED $\mathrm{BaTiO}_{3}$ FILMS 


\title{
STRUCTURAL AND TRANSPORT PROPERTIES OF EPITAXIAL NIOBIUM-DOPED $\mathrm{BaTiO}_{3}$ FILMS
}

\author{
By \\ YANG SHAO, M.S.
}

\begin{abstract}
A Thesis
Submitted to the School of Graduate Studies in Partial Fulfilment of the Requirements

for the Degree of

Doctor of Philosophy
\end{abstract}

McMaster University

(C) Copyright by Yang Shao, January 2009 
DOCTOR OF PHILOSOPHY (2009)

(Department of Materials Science and Engineering)
McMaster University

Hamilton, Ontario
TITLE:

AUTHOR:

SUPERVISORS:

NUMBER OF PAGES: $\quad$ xvi, 219
Structural and Transport Properties of Epitaxial

Nb-doped $\mathrm{BaTiO}_{3}$ Films

Yang Shao, M.S.

(Tsinghua University)

Gianluigi A. Botton and John S. Preston 


\section{Abstract}

Highly orientated $\mathrm{BaTi}_{1-x} \mathrm{Nb}_{x} \mathrm{O}_{3}$ thin films, spanning the entire range of $\mathrm{x}$, have been successfully deposited on (001) $\mathrm{MgAl}_{2} \mathrm{O}_{4}$ substrates by the pulsed laser deposition (PLD) method. The structure of the films is characterized with a range of techniques. It is found that increasing $x$ gives rise to a $\mathrm{Ti}^{4+}$ to $\mathrm{Ti}^{3+}$ transformation in the oxidation state accompanied by increased conductivity with a semiconductormetal transition near $x=0.2$. Temperature dependent magnetic measurements show an anomalous rise in the spin moment. In order to further reduce the lattice mismatch and keep the conductivity at the same time, a partial strontium-for-barium substitution, $\left(\mathrm{Ba}_{1-y} \mathrm{Sr}_{y}\right) \mathrm{Ti}_{0.5} \mathrm{Nb}_{0.5} \mathrm{O}_{3}$ with $y=0,0.4,0.5$ and 0.6 , were used. Such a substitution provides a means for independently tuning the lattice parameter and conductivity over a significant range of compositions. The $y=0.6$ composition show a sharp interface with flawless epitaxy and good quality films. We attribute the improvements in the film quality to a decrease in the lattice misfit strain made possible through the superior lattice match to the substrate obtained through strontium substitution. Electronic structure calculations were carried out by the $1^{\text {st }}$ principle method using the WIEN2k program in order to understand the electronic structure 
of these compounds. Based on the assumed ordered structures, the Fermi level of $\mathrm{BaTi}_{1-x} \mathrm{Nb}_{x} \mathrm{O}_{3}$ gradually moved to the lower energies as $x$ increase, while the valence bands were not significantly altered with the $\mathrm{Nb}$ ions substitutions. The fraction of each $\mathrm{Ti}^{4+}$ and $\mathrm{Ti}^{3+}$ component in $\mathrm{BaTi}_{1-x} \mathrm{Nb}_{x} \mathrm{O}_{3}$ samples was extracted by the linear profile fitting of the corresponding $\mathrm{Ti}-\mathrm{L}_{2,3}$ edge obtained by the electron energy loss spectra. The fitting results indicate a high fraction of $\mathrm{Ti}^{3+}$ is present than excepted as $\mathrm{Nb}$ content increase, which could arise from the loss of oxygen stoichiometry. The electron energy loss spectra of the O-K edge is analyzed by comparison to the partial density of states calculation. The evolutions of O-K edge features are explained in terms of the decrease of the Ti $3 d$ band contribution and the increase of the $\mathrm{Nb} 4 d$ band contribution as the $\mathrm{Nb}$ content increase. 


\section{Acknowledgements}

The path towards this thesis spans four and a half years of work and the contribution of many people. I would like to thank everyone who has helped and inspired me during my doctoral study.

The first person I would like to thank is my primary supervisor, Dr. Gianluigi Botton. Since I started to work on this project in September 2004, he continued encouragement, invaluable suggestions, and financial support helped me to complete this Ph.D. work.

I would also like to thank my co-supervisor, Dr. John Preston, for the helpful discussion and opportunity to complete this work.

I wish to thank Dr. Rob Hughes for his interest, passion, and help in thin film growth and resistivity measurements. His hard work ensured an excellent source of high quality films I could study in details. I also want to thank him for help in the editing process of submitted papers. 
I wish to thank Dr. Antoni Dabkowski for the useful discussion about the ceramic target synthesis. He also taught me how to use the Guinier camera for X-ray diffraction, and let me use his lab to prepare ceramic target samples.

I would like to thank Dr. Christian Maunders for the help with the acquisition of the EELS spectra and many discussion about the linear fitting method. His generous help, often late of night, helped me in the acquisition of EEL spectra.

Dr. Guillaume Radtke first introduced me to the WIEN2k and Multiplet program. He led me into the field of $1^{\text {st }}$ principle calculation. I want to thank him for the advice of EELS core-hole effect calculation, $\mathrm{LDA}+\mathrm{U}$ calculation and crystal field multiplet calculation.

I am also very grateful to Mr. Fred Pearson and Mr. Andy Duft for the help in TEM training and sample preparation; Mr. Wen He Gong and Dr. James Britten for the help of X-ray diffraction and useful discussion about pole figures; Lan Yun (Shery) Chang and Sorin Lazar for the helpful discussions regarding the HRTEM and HAADF images.

Finally, I would like to thank my beloved wife Hongxia Ma. Her understanding and support encourage me to pursue my doctoral degree. A special thought is also devoted to my parents for the never-ending support.

Hamilton, January 2009 Yang Shao 


\section{Contents}

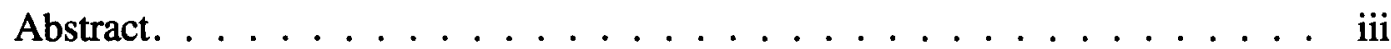

Acknowledgements . . . . . . . . . . . . . . . v

Table of Contents . . . . . . . . . . . . . . . . . . x

List of Figures $\ldots \ldots \ldots \ldots \ldots \ldots \ldots \ldots \ldots \ldots$ xiv

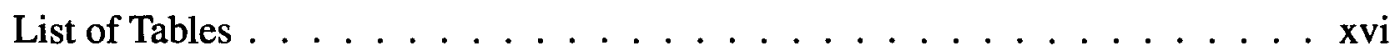

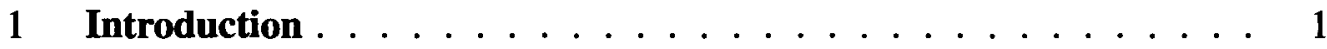

2 Literature Review . . . . . . . . . . . . . . . . . . . . 7

2.1 Oxide materials. . . . . . . . . . . . . . 7

2.2 Perovskite oxide and stability . . . . . . . . . . . . 9

2.3 B-site substitution . . . . . . . . . . . . 11

2.4 Oxygen vacancies and doping effect $\ldots \ldots \ldots \ldots$

2.5 Metal-insulator transition . . . . . . . . . . . . . . 16

2.5.1 Band gap insulator . . . . . . . . . . . . . . 16

2.5.2 Mott-Hubbard gap . . . . . . . . . . . . . . 17

2.5.3 Anderson localization . . . . . . . . . . . . . 20

2.5.4 Weak localization and conductance fluctuations. . . . . . 21

2.6 Conduction mechanism . . . . . . . . . . . . . . 22

2.6.1 Thermal excitation . . . . . . . . . . . . . 22

2.6 .2 Variable-range hopping. . . . . . . . . . . . . . 23

2.6 .3 Polaron conduction. . . . . . . . . . . . . . . 25 
2.7 Literature review of $\mathrm{Nb}$-doped barium titanate $\ldots \ldots \ldots$

2.7.1 Ceramic specimen . . . . . . . . . . . . . 31

2.7 .2 Thin films . . . . . . . . . . . . . 35

3 Characterization Techniques . . . . . . . . . . . 43

3.1 X-ray diffraction . . . . . . . . . . . . . . 44

3.1.1 Traditional powder X-ray diffraction . . . . . . . . . . 44

3.1.2 Two-dimensional X-ray diffraction . . . . . . . . . . 45

3.1.3 Asymmetric rocking curve X-ray diffraction . . . . . . 4 46

3.2 Electron microscopy . . . . . . . . . . . . . . . . 48

3.3 Electron energy loss spectroscopy. . . . . . . . . . . . . . . . 49

3.4 The van der Pauw method . . . . . . . . . . . . 52

4 Electronic Structure Calculation Techniques . . . . . . . . . . . 55

4.1 WIEN2k . . . . . . . . . . . . . . 56

4.2 Multiplet methods . . . . . . . . . . . . . 66

4.2.1 Basis aspects . . . . . . . . . . . . . . 67

4.2 .2 Atomic multiplets . . . . . . . . . . . . . . 69

4.2.3 Matrix elements . . . . . . . . . . . . . . . . 71

4.2.4 Spectra described with atomic multiplets . . . . . . . 72

4.2.5 The crystal field multiplet . . . . . . . . . . . . 74

5 Thin Films Growth Technique. . . . . . . . . . . . . . . 81

5.1 Preparation of ceramic targets. . . . . . . . . . . . 82

5.2 The pulse laser deposition technique $\ldots \ldots \ldots$. . . . . 83

6 Substrate $\mathrm{MgAl}_{2} \mathbf{O}_{4} \ldots \ldots \ldots \ldots \ldots \ldots$

6.1 The surface roughness $\ldots \ldots \ldots \ldots \ldots$

6.2 Crystal structure . . . . . . . . . . . . . . . 90 90

$7 \mathrm{BaTi}_{1-x} \mathrm{Nb}_{x} \mathrm{O}_{3}$ Thin Films on (001) $\mathrm{MgAl}_{2} \mathrm{O}_{4}$ Substrate $\ldots \ldots \ldots 91$

7.1 Phase purity . . . . . . . . . . . . . 92

7.2 Structure and lattice parameter . . . . . . . . . . . . 94

7.3 Grain orientation . . . . . . . . . . . . . . . . 97 
7.4 Twin Structure . . . . . . . . . . . . . . . . . . 102

7.5 TEM analysis. . . . . . . . . . . . . . . . . . . . 104

$7.6 \mathrm{Nb}$ ions distribution by diffraction pattern . . . . . . . . . . 105

7.7 Valence state analysis by EELS . . . . . . . . . . . . 108 7.7.1 Ti-L 2,3 edge . . . . . . . . . . . . . . . 108 7.7 .2 O-K edges . . . . . . . . . . . . . . . . 117

7.8 Resistivity measurement . . . . . . . . . . . . . 121

$7.9 \quad I-V$ curve . . . . . . . . . . . . . . . . . 123

7.10 Hall coefficient measurement . . . . . . . . . . . . . 123

7.11 A possible 2D Percolation phenomenon . . . . . . . . . . 128

7.12 Magnetic properties and possible superconducting phase . . . 131

7.13 Electron density of states calculation . . . . . . . . . . 138

7.14 Electron density calculation. . . . . . . . . . . . . . 140

7.15 Band structure and Fermi surface . . . . . . . . . . . 144

$8 \mathrm{BaTi}_{0.5} \mathrm{Nb}_{0.5} \mathrm{O}_{3}$ Thin Films on (001), (011) and (111) $\mathrm{MgAl}_{2} \mathrm{O}_{4}$ Substrate . . . . . . . . . . . . . . . . . . . . . 149

$8.1 \quad \mathrm{XRD}$ results $\ldots \ldots \ldots \ldots \ldots \ldots \ldots$

8.2 Thin film surface morphology. . . . . . . . . . . . 151

8.3 TEM analysis. . . . . . . . . . . . . . . 153

8.4 Resistivity . . . . . . . . . . . . . . . . 155

$9 \mathrm{Ba}_{1-y} \mathrm{Sr}_{y} \mathrm{Ti}_{0.5} \mathrm{Nb}_{0.5} \mathrm{O}_{3}$ Thin Films on $\mathrm{MgAl}_{2} \mathrm{O}_{4}$ Substrate $\ldots \ldots 157$

9.1 Phase purity and lattice parameters . . . . . . . . . . 158

9.2 Grain orientation . . . . . . . . . . . . . . . . 161

9.3 Surface roughness $\ldots \ldots \ldots \ldots$

9.4 Deposition temperature effect . . . . . . . . . . . . . 166

9.5 Microstructure and interface analysis . . . . . . . . . . 170

9.6 Hall coefficient measurement . . . . . . . . . . . . . 171

9.7 Resistivity measurement . . . . . . . . . . . . . . . 173

10 Quantification of the Ti oxidation state . . . . . . . . . . . . . 177 
11 Conclusion. . . . . . . . . . . . . . . . . . . . 189

Appendix A Grain Orientation of $\mathrm{BaTi}_{1-x} \mathbf{N b}_{x} \mathrm{O}_{3}$ Thin Films . . . . 193

Appendix B Pole Figures of $\left(\mathrm{Ba}_{1-y} \mathbf{S r}_{y}\right) \mathbf{T i}_{0.5} \mathbf{N b}_{0.5} \mathbf{O}_{3}$ Thin Films . . . . 203

Appendix C Lorentzian Broadening Program . . . . . . . . . . . 207

References . . . . . . . . . . . . . . . . . . . . . . 208 


\section{List of Figures}

2.1 Ideal cubic perovskite structure . . . . . . . . . . . . . . 9

2.2 Three B-site sublattice types for $1: 1$ ordered perovskites $\ldots \ldots \ldots \ldots 12$

2.3 Structural dependence on charge difference and ionic radius difference . . . . 14

2.4 Relative energies of oxygen and metal states . . . . . . . . . . . . 19

2.5 Defect concentrations dependence on $\mathrm{Nb}$ concentration . . . . . . . . 33

$2.6 \mathrm{BaTi}_{1-x} \mathrm{Nb}_{x} \mathrm{O}_{3}(00 l)$ thin films lattice parameters. . . . . . . . . . 38

2.7 Temperature dependence of resistivity of $\mathrm{BaTi}_{1-x} \mathrm{Nb}_{x} \mathrm{O}_{3} \ldots \ldots \ldots$

2.8 Resistivity and reciprocal of mobility of the thin films at $x=0.5 \ldots 41$

2.9 Conduction mechanism for different $\mathrm{Nb}$ concentration thin films. . . . . . . 41

3.1 3D X-ray diffaction patterns from a powder sample . . . . . . . . . 45

3.2 Five major units in a GADDS system . . . . . . . . . . . . . 46

3.3 Sample rotation and translation in the laboratory system. . . . . . . . . 46

3.4 Detector position in the laboratory system $\mathrm{X}_{L} \mathrm{Y}_{L} \mathrm{Z}_{L} \ldots \ldots \ldots \ldots \ldots$

3.5 Typical asymmetric rocking curve results $\ldots \ldots \ldots \ldots \ldots$

3.6 A schematic representation of a typical electron energy loss spectrum . . . . 50

3.7 Contact placements for Hall and Resistivity measurement . . . . . . . . 53

4.1 Flow chart of self-consistent procedure . . . . . . . . . . . 61

4.2 Division of a unit cell in muffin tin regions and the interstitial region . . . . 63

$4.3 \mathrm{~d}$ orbitals splitting in $\mathrm{O}_{h}$ crystal field. . . . . . . . . . 76 
4.4 Crystal field multiplets prediction comparing to the experimental result . . . . 78

5.1 The schematic representation of a pulsed laser deposition instrument . . . . . 84

6.1 Surface morphology of $(001) \mathrm{MgAl}_{2} \mathrm{O}_{4}$ substrate. . . . . . . . . . . . . 89

$6.2 \mathrm{MgAl}_{2} \mathrm{O}_{4}$ substrate CBED patterns . . . . . . . . . . . . . . 90

$7.1 \theta-2 \theta$ XRD spectra for the $2 \theta$ range near the film's (002) peak . . . . . . . 93

7.2 The large $2 \theta$ range XRD spectra for $\mathrm{BaTi}_{1-x} \mathrm{Nb}_{x} \mathrm{O}_{3}$ thin films . . . . . . . . 94

7.3 Lattice parameter $c$ as a function of $\mathrm{Nb}$ concentration $\mathrm{x}$. . . . . . . . . . 96

7.4 Films $\{001\}$ pole figures for $\mathrm{BaTi}_{1-x} \mathrm{Nb}_{x} \mathrm{O}_{3}$ thin film at $x=0.5 \ldots$. . . . 98

7.5 Films $\{001\}$ pole figures for $\mathrm{BaTi}_{1-x} \mathrm{Nb}_{x} \mathrm{O}_{3}$ thin film at $x=0.5$ and 1.0. . . 100

7.6 Nine possible grain orientations confirmed in $\{011\}$ pole figures. . . . . . 101

7.7 The orientation relationship between one minor and major grain orientation. . 102

7.8 Twin structure found by diffraction pattern along (110) zone axis . . . . . 103

7.9 TEM bright field image of $\mathrm{BaTi}_{1-x} \mathrm{Nb}_{x} \mathrm{O}_{3}$ thin film . . . . . . . . . . . 106

7.10 EDS analysis of $\mathrm{BaTi}_{1-x} \mathrm{Nb}_{x} \mathrm{O}_{3}$ thin film at $x=0.50$ composition . . . . . . 107

7.11 CBED patterns and simulated diffraction patterns. . . . . . . . . . . 109

7.12 Zero loss peak of a high-resolution energy-loss spectrometer . . . . . . . 110

$7.13 \mathrm{Ti}-L_{2,3}$ edge spectra for $\mathrm{BaTi}_{1-x} \mathrm{Nb}_{x} \mathrm{O}_{3}$ thin films . . . . . . . . . . . . . . 112

$7.14 \mathrm{Ti}-\mathrm{L}_{2,3}$ edge spectra for polycrystalline $\mathrm{BaTi}_{1-x} \mathrm{Nb}_{x} \mathrm{O}_{3} \ldots \ldots$. . . . . . . . 114

7.15 Multiplet calculation for $\mathrm{Ti}^{4+}$ and $\mathrm{Ti}^{3+}$ in $\mathrm{O}_{h}$ symmetry. . . . . . . . . 115

$7.16 \mathrm{Nb}-L_{3}$ edge XANES of polycrystalline $\mathrm{BaTi}_{1-x} \mathrm{Nb}_{x} \mathrm{O}_{3} \ldots \ldots \ldots$

7.17 O-K edge ELNES of $\mathrm{BaTi}_{1-x} \mathrm{Nb}_{x} \mathrm{O}_{3} \ldots \ldots \ldots$

7.18 Site projected PDOS of $\mathrm{BaTiO}_{3}$ and $\mathrm{BaNbO}_{3}$. . . . . . . . . . . 120

7.19 Resistivity of $\mathrm{BaTi}_{1-x} \mathrm{Nb}_{x} \mathrm{O}_{3}$ as a function of $\mathrm{Nb}$ concentration. . . . . . . . 122

$7.20 I-V$ curves for $\mathrm{BaTi}_{1-x} \mathrm{Nb}_{x} \mathrm{O}_{3}$ films . . . . . . . . . . . . . . . . . . . . 124

7.21 A schematically illustration of charge carries type and densities change. . . . 126

7.22 Charge density as $\mathrm{Nb}$ content increase. . . . . . . . . . . . . 127

7.23 Conductivity variation with different $\mathrm{Nb}$ concentrations . . . . . . . . . . . 129 
7.24 Temperature dependent spin moments . . . . . . . . . . . . . . . . 132

7.25 Magnetic properties of $\mathrm{x}=0.50$ thin film . . . . . . . . . . . . 135

$7.26 \mathrm{x}=0.50$ film under different magnetic fields . . . . . . . . . . . . . 136

7.27 The resisitivity dependence of temperature for $x=0.50$ film . . . . . . . 137

$7.282 \times 2 \times 2$ ordered structure for calculation . . . . . . . . . . . . . . 139

7.29 The calculated electron density of state (DOS) of $\mathrm{BaTi}_{1-x} \mathrm{Nb}_{x} \mathrm{O}_{3}$ thin film . . 140

7.30 DOS of $x=0.50$ conducting film around Fermi level . . . . . . . . . . . . 142

7.31 Conduction electron density on (001) plane for $x=0.5$. . . . . . . . . . . 142

7.32 Site projected PDOS of $\mathrm{BaTi}_{0.5} \mathrm{Nb}_{0.5} \mathrm{O}_{3} \ldots \ldots$. . . . . . . . . . . 143

7.33 Band structure of $\mathrm{BaTi}_{0.5} \mathrm{Nb}_{0.5} \mathrm{O}_{3} \ldots \ldots \ldots$

7.34 Fermi surface of $\mathrm{x}=0.5$ sample . . . . . . . . . . . . . . . 147

8.1 XRD results of $\mathrm{BaTi}_{0.5} \mathrm{Nb}_{0.5} \mathrm{O}_{3}$ thin films on (001), (011) and (111) $\mathrm{MgAl}_{2} \mathrm{O}_{4} \quad 150$

8.2 SEM images of $\mathrm{BaTi}_{0.5} \mathrm{Nb}_{0.5} \mathrm{O}_{3}$ deposited on (011) and (111) $\mathrm{MgAl}_{2} \mathrm{O}_{4} \ldots \ldots$

8.3 TEM results of $(001),(011)$ and (111) orientated $\mathrm{BaTi}_{0.5} \mathrm{Nb}_{0.5} \mathrm{O}_{3}$ films . . . . 154

8.4 Resistivity of $(001),(011)$ and (111) orientated $\mathrm{BaTi}_{0.5} \mathrm{Nb}_{0.5} \mathrm{O}_{3}$ films . . . . . 155

9.1 XRD results of $\left(\mathrm{Ba}_{1-y} \mathrm{Sr}_{y}\right) \mathrm{Ti}_{0.5} \mathrm{Nb}_{0.5} \mathrm{O}_{3}$ films $\ldots \ldots \ldots 0$

$9.2\{111\}$ pole figure of BSTN. . . . . . . . . . . 163

$9.3\{111\}$ pole figure of BSTN. . . . . . . . . . . . 164

9.4 SEM images of BSTN thin film. . . . . . . . . . . . . . 165

9.5 SEM images of $\left(\mathrm{Ba}_{0.4} \mathrm{Sr}_{0.6}\right) \mathrm{Ti}_{0.5} \mathrm{Nb}_{0.5} \mathrm{O}_{3}$ deposited at different temperatures 167

9.6 Structure zone diagram for evaporated metal films . . . . . . . . . . . . . 169

9.7 TEM results of $\left(\mathrm{Ba}_{1-y} \mathrm{Sr}_{y}\right) \mathrm{Ti}_{0.5} \mathrm{Nb}_{0.5} \mathrm{O}_{3}$ thin films . . . . . . . . . . 172

9.8 Resistivity of $\left(\mathrm{Ba}_{1-y} \mathrm{Sr}_{y}\right) \mathrm{Ti}_{0.5} \mathrm{Nb}_{0.5} \mathrm{O}_{3}$ thin films as a function of temperature . 174

9.9 Temperature dependences of resistivity of $\left(\mathrm{Ba}_{1-y} \mathrm{Sr}_{y}\right) \mathrm{Ti}_{0.5} \mathrm{Nb}_{0.5} \mathrm{O}_{3}$ thin films .175

10.1 The shape change of $\mathrm{Ti}_{2,3}$ edges . . . . . . . . . . . . . . 181

10.2 Background subtraction method for $\mathrm{Ti}_{-1,3}$ edge . . . . . . . . . . . . 183

10.3 Ti- $\mathrm{L}_{2,3}$ edge fitting results . . . . . . . . . . . . . . . . 185 
10.4 The average $3 d$ occupancy of $\mathrm{Ti}$ ions $\ldots \ldots \ldots \ldots$

A.1 The coordination relationship of the film and 2D XRD sample state . . . 194

A.2 Films $\{111\}$ pole figures for $\mathrm{BaTi}_{1-x} \mathrm{Nb}_{x} \mathrm{O}_{3}$ thin film at $\mathrm{x}=0.5$ and $1.0 \ldots \ldots 195$

A.3 Films $\{001\}$ pole figures for $\mathrm{BaTi}_{1-x} \mathrm{Nb}_{x} \mathrm{O}_{3}$ thin film at $\mathrm{x}=0.5$ and $1.0 \ldots \ldots 197$

A.4 Nine possible grain orientations found in $\{001\}$ pole figures. . . . . . . 200

A.5 Nine possible grain orientations confirmed in $\{011\}$ pole figures. . . . . 202

B.1 (001) pole figure of $\left(\mathrm{Ba}_{1-y} \mathrm{Sr}_{y}\right) \mathrm{Ti}_{0.5} \mathrm{Nb}_{0.5} \mathrm{O}_{3}$ thin films $\ldots \ldots \ldots \ldots 20 \ldots$

B.2 (011) pole figure of $\left(\mathrm{Ba}_{1-y} \mathrm{Sr}_{y}\right) \mathrm{Ti}_{0.5} \mathrm{Nb}_{0.5} \mathrm{O}_{3}$ thin films $\ldots \ldots \ldots \ldots$ 


\section{List of Tables}

2.1 Examples of 1:1 B-Site ordered perovskite. . . . . . . . . . . . . 29

2.2 Resistivity, carrier density, and Hall mobility of $\mathrm{BaTi}_{1-x} \mathrm{Nb}_{x} \mathrm{O}_{3}$ thin films. . . 39

4.118 term symbols for $3 d^{1} 4 d^{1}$ configuration . . . . . . . . . . . . . . 70

4.2 All allowed transitions for $3 d^{N}$ systems . . . . . . . . . . . . . . . 74

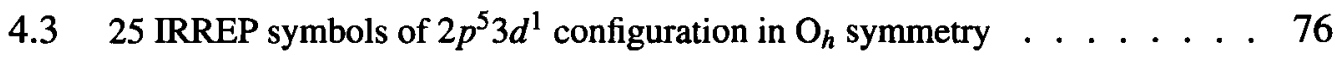

4.4 Multiplication table of IRREP for $\mathrm{O}_{h}$ symmetry . . . . . . . . . . . . . 77

7.1 The value of lattice parameter $c$ of $\mathrm{BaTi}_{1-x} \mathrm{Nb}_{x} \mathrm{O}_{3}$ thin films. . . . . . . 95

7.2 Integration range for each pole figure . . . . . . . . . . . . . 97

7.3 Unit cell and atomic parameters for $\mathrm{BaTiO}_{3}$ and $\mathrm{BaNbO}_{3}$. . . . . . . . . . 119

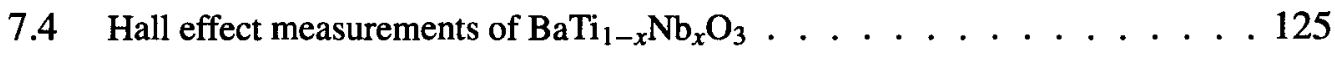

7.5 Fitting parameters for $2 \mathrm{D}$ percolation phenomena . . . . . . . . . 130

8.1 The value of lattice parameter $c$ of different orientated $\mathrm{BaTi}_{0.5} \mathrm{Nb}_{0.5} \mathrm{O}_{3}$ films on $\mathrm{MgAl}_{2} \mathrm{O}_{4}$ substrate deposition at $850^{\circ} \mathrm{C}$ by assuming a cubic structure.. . . 151

9.1 Lattice parameter $c$ of $\left(\mathrm{Ba}_{1-y} \mathrm{Sr}_{y}\right) \mathrm{Ti}_{0.5} \mathrm{Nb}_{0.5} \mathrm{O}_{3}$ thin films . . . . . . . . 160

9.2 Integration range for pole figures of BSTN. . . . . . . . . . . . 162

9.3 Hall effect measurements of $\left(\mathrm{Ba}_{1-y} \mathrm{Sr}_{y}\right) \mathrm{Ti}_{0.5} \mathrm{Nb}_{0.5} \mathrm{O}_{3} \ldots \ldots . . . \ldots 173$

10.1 The fractions of $\mathrm{Ti}^{3+}$ and $\mathrm{Ti}^{4+}$ components . . . . . . . . . . 186 
PhD Thesis—Yang Shao-McMaster University-Materials Science and Engineering 2009

A.1 Integration range for each pole figure . . . . . . . . . . . . . . 194 


\section{Chapter 1}

\section{Introduction}

Oxides exhibit a wide variety of electrical properties. They can be excellent insulators, semiconductors or metals and even superconductors. This broad range of transport properties, combined with their interesting magnetic behavior have given rise to exciting ideas in the field of industrial applications as it becomes possible to design new properties by engineering their structure and composition. Besides practical applications, the complex electron correlation effects exhibited by these materials have attracted the interest of thousands of physicists throughout the world for many decades. A large number experimental results and theoretical investigations have appeared in the literature and have challenged our understanding of solid state physics (Dagotto, 2005).

$\mathrm{ABO}_{3}$-type perovskite oxides are particular interesting framework materials that have promising electronic applications that can be tuned by variations of their 
compositions, and have demonstrated plethora of exceptional properties, such as giant magnetoresistivity, superconductivity and ferroelectricity. More recently, the interest in these materials has grown even further based on remarkable characteristics of their interface (Huijben et al., 2006). Within the family of perovskites , $\mathrm{Pb}\left(\mathrm{Zr}_{x} \mathrm{Ti}_{1-x}\right) \mathrm{O}_{3}$ (PZT) thin films are some of the most promising materials for nonvolatile memory devices, and a novel relaxor ferroelectric $\mathrm{Pb}\left(\mathrm{Zr}_{x} \mathrm{Ti}_{1-x}\right) \mathrm{O}_{3} / \mathrm{PbTiO}_{3}$ (PZT/PT) has been targeted for the next generation ferroelectric applications. As the demand for miniaturization of devices by the micro-electronic industry continues to grow, thin films making use of the functional properties of these materials are more and more important. High performance electronic device requires the fabrication of a high quality thin film device together with suitable electrode materials. As a consequence, for such functional applications, the bottom electron-conducting materials also become more and more critical. This is not only relevant because such materials act as the substrate of the devices and thus ultimately control the quality of the functional oxide thin films, but also because they can affect the properties of the devices by inducing strain to the functional oxide thin films themselves. Therefore a suitable conducting substrate for the epitaxial thin film growth of functional electronic devices is extremely important.

Generally, the ideal substrate for a device should be lattice matched, chemically compatible, thermal expansion matched, and should show no structural phase transitions between the film growth temperature and the device use temperature (Chakoumakos et al., 1998). For the bottom electrode materials, we have to consider another one major requirement: good conductivity. The most widely used electrode materials are metals, such as $\mathrm{Pt}, \mathrm{Au}$ and $\mathrm{Cu}$. However, metal electrodes induce se- 
vere fatigue problems in the ferroelectric devices. The switchable polarization will decrease during the switching cycles. This will significantly impair the working life of the ferroelectrics. Many models and mechanisms have been proposed to explain these effects. Most of these are related to the loss of polarization, the behavior of oxygen vacancies in the thin film or around the interface. Obviously, the metal electrodes themselves cannot compensate for the oxygen vacancies in the functional film. In order to overcome the fatigue problems, oxide-based electrodes have been proposed. For example, it is believed that oxygen vacancies in the PZT thin films can be compensated by the oxygen in the oxide electrodes (Ramesh et al., 1992; Lee et al., 1995; Paz de Araujo et al., 1996). This will reduce the fatigue problems and extend the device working life.

Although oxides materials are not commonly well known for their ability to conduct electrons, nevertheless, they have become indispensable electrical contacts in many devices, with indium tin oxide (ITO) being the most prominent example. The incorporation of oxide conductors into a device has always relied on the fact that they offer reasonable conductivity, while simultaneously providing a secondary property which is essential to the application: for the case of ITO the property is optical transparency. Another function often required of oxide conductors is the ability to facilitate a heteroepitaxial relationship with a material requiring a bottom electrode, as is often the case for oxides showing ferroelectric, piezoelectric or relaxor properties. Materials such as $\mathrm{BaTiO}_{3}, \mathrm{~Pb}\left(\mathrm{Zr}_{x} \mathrm{Ti}_{1-x}\right) \mathrm{O}_{3} / \mathrm{PbTiO}_{3}(\mathrm{PZT} / \mathrm{PT})$ and $\mathrm{Pb}\left(\mathrm{Mg}_{1 / 3} \mathrm{Nb}_{2 / 3}\right) \mathrm{O}_{3} / \mathrm{PbTiO}_{3}$ ( $\mathrm{PMN}-\mathrm{PT}$ ) all fall into this category. The electrode materials often used for these purposes, however, have lattice constants which are typically less than $4 \AA$, while the aforementioned dielectric materials all have lat- 
tice constants above this value. Examples of oxide electrode materials commonly used are $\mathrm{IrO}_{2}$ (tetragonal a=4.5 $\AA, \mathrm{c}=3.15 \AA$ (Liu et al., 2004), $\mathrm{SrRuO}_{3}$ (pseudocubic cubic a=3.9 $\AA$ (Eom et al., 1993), $\mathrm{LaNiO}_{3}$ (pseudocubic a=3.84 $\AA$ ) (Detalle \& Remiens, 2008) and $\mathrm{La}_{0.5} \mathrm{Sr}_{0.5} \mathrm{CoO}_{3}$ (cubic a=3.80 $\AA$ ) (Span et al., 1999; Chen et al., 1999). Most of the frequently used oxide substrates do not fair much better in this regard, as many of them are perovskite materials developed for the deposition of high temperature superconductors, where smaller lattice constants are required (Schlom et al., 2007). The only cubic oxide substrate with a lattice constant greater than $4 \AA$ which has been widely adopted is $\mathrm{MgO}$, but with $\mathrm{a}=4.22 \AA$ it presents a lattice constant that is considerably larger than both the contact layers and dielectrics being deposited on it.

In this thesis we demonstrated that $\mathrm{Nb}$-doped barium titanate is one promising candidate for the better lattice match with potential PZT/PT ferroelectric device. $\mathrm{Nb}$-doped barium titanate also exhibits rich electronic and magnetic properties that need to be better understood. In the literature, most work on Nb-doped barium titanate has focused on low $\mathrm{Nb}$ content bulk samples due to the difficulty of incorporating $\mathrm{Nb}$ into $\mathrm{BaTiO}_{3}$ lattice. There are also very few reports on the thin films of this material (Nagano et al., 1998; Khan et al., 1999; Gasparov et al., 2001; Lemée et al., 2002; Liu et al., 2005; Huang et al., 2006), but most of them have not focused on the high $\mathrm{Nb}$ content samples so far.

In this $\mathrm{Ph} . \mathrm{D}$. project, the aim is to cover the knowledge gap for this material for potential applications as conducting electrode by studying the growth and transport properties of $\mathrm{Nb}$-doped $\mathrm{BaTiO}_{3}$ thin films. We demonstrated that that low oxy- 
gen partial pressure was the key to the ability to insert more $\mathrm{Nb}$ into the $\mathrm{BaTiO}_{3}$ lattice and we synthesized, for the first time, a series of $\mathrm{BaTi}_{1-x} \mathrm{Nb}_{x} \mathrm{O}_{3}$ spanning the whole range of $x$, and demonstrated that no solubility limit exsit for this material. We also demonstrated that, with increased $\mathrm{Nb}$ content the material becomes increasingly conducting with a transition to metallic behavior near $\mathrm{x}=0.2$, a value consistent with other reports on bulk (Marucco et al., 1997) and thin film materials.(Liu et al., 2005) Over this same range the lattice constants varied from 4.014 to $4.142 \AA$. However, the values of $\mathrm{x}$ which match most closely to the substrate's unit cell are those not showing metallic behavior. Such a situation appears to reduce the effectiveness of $\mathrm{BaTi}_{1-x} \mathrm{Nb}_{x} \mathrm{O}_{3}$ as a bottom electrode material. This issue was later resolved in our work through a partial strontium-for-barium substitution in the films. Such a substitution is well known to decrease the lattice parameter in the $\left(\mathrm{Ba}_{1-y} \mathrm{Sr}_{y}\right) \mathrm{TiO}_{3}$ system (Arya et al., 2003; Liou \& Chiou, 1997). At the same time, the material is expected to maintain the perovskite's A-site valence state, and hence this substitution does not significantly alter the electronic transport properties (Inoue et al., 1995; Lee et al., 2002). Thus, a similar substitution in the thin films of $\mathrm{BaTi}_{0.5} \mathrm{Nb}_{0.5} \mathrm{O}_{3}$ system resulted in a conducting oxide, providing a superior epitaxial relationship with the $(001) \mathrm{MgAl}_{2} \mathrm{O}_{4}$ substrate. In a more general sense this thesis demonstrated that such substitution, together with the $\mathrm{Nb}$ doping provide a means for independently tuning the lattice parameter and conductivity over a significant range of values.

The structure characterization and physical properties of the $\mathrm{BaTi}_{1-x} \mathrm{Nb}_{x} \mathrm{O}_{3}$ and $\left(\mathrm{Ba}_{1-y} \mathrm{Sr}_{y}\right) \mathrm{Ti}_{0.5} \mathrm{Nb}_{0.5} \mathrm{O}_{3}$ thin films were carried out with a range of techniques: $\mathrm{X}$ ray $\theta-2 \theta$ scan, 2 dimensional $X$-ray diffraction, atomic force microscopy (AFM), 
scanning electron microscopy (SEM), energy dispersive X-ray spectrometry (EDS), transmission electron microscopy (TEM) (bright field imaging (BF), selected area electron diffraction (SAD), high-resolution imaging and electron energy loss spectroscopy (EELS), van der Pauw resistivity measurement, $I-V$, hall coefficient and magnetic properties measurements.

In addition to the experiments work, a useful experimental method was also developed. From the analysis of electron energy loss spectra, the transition metal valence fractions of ions for the mixed-valence compound were retrieved. In order to support our experimental data with a better insight of the electronic structure of this material, we made extensive use of first-principle calculations using density functional theory through the use of the full-potential linearized augmented plane-wave program WIEN2k. With these calculations, we interpreted not only the details of EELS O-K edges spectra but we also gained a better understanding of the electronic properties of $\mathrm{BaTi}_{1-x} \mathrm{Nb}_{x} \mathrm{O}_{3}$.

In this thesis, I will initially give the necessary background to understand the properties of conducting oxide, the metal-insulator transition and conduction mechanisms (Chapter 2 section 2.1-2.6), and subsequently present a full-scale literature review of Nb-doped barium titanate (Chapter 2 section 2.7); then describe the film characterization techniques (Chapter 3), electronic structure calculation methods (Chapter 4), and our films deposition process (Chapter 5); finally discuss the results of structural and transport properties of this interesting material $\mathrm{BaTi}_{1-x} \mathrm{Nb}_{x} \mathrm{O}_{3}$ (Chapter 7) with the extension to the $\left(\mathrm{Ba}_{1-y} \mathrm{Sr}_{y}\right) \mathrm{Ti}_{0.5} \mathrm{Nb}_{0.5} \mathrm{O}_{3}$ (Chapter 9). The conclusions and further work will be covered in the final chapter of this thesis. 


\section{Chapter 2}

\section{Literature Review}

Oxides, especially perovskite oxides, have been a hot topic for decades. Attentions are attracting from electronic industry to fundamental scientific research. In this chapter, a brief introduction of oxide materials is reviewed first and followed by a detail description of perovskite oxide and B-site substitution. Then metal-insulator transition and conduction mechanism are discussed. We end with current literature review of $\mathrm{BaTiO}_{3}: \mathrm{Nb}$.

\subsection{Oxide materials}

In our modern industry, oxides play a central role from the oxidation and reduction of oxides in iron and aluminum industry, to the manufacture of paints, catalysts, and digital recording media (Spence, 2003). Most alkali and alkaline-earth metal oxides 
are insulating ionic compounds with simple electronic structures due to the highly ionized nature of the metal ions; however, the bonding nature between the transition metal and oxygen ions is not that straightforward, resulting the complex electronic structures and rich optical and electrical properties. Transition metal oxides together with their defects have been one of the central topics in condensed matter physics for decades, from the high temperature superconductor $\left(\mathrm{YBa}_{2} \mathrm{Cu}_{3} \mathrm{O}_{7}\right)$ and colossal magnetoresistance materials $\left(\mathrm{La}_{1-x} \mathrm{Ca}_{x} \mathrm{MnO}_{3}\right)$ to the new promising relaxor (PZT/PT). Many researchers believe that the next generation of electronic devices will be focused on oxides.

There are three-transition series forming the short groups of elements in the periodic table that form oxides with extraordinarily varied chemical and physical properties of their compound. A few of them are volatile compounds, for example $\mathrm{OsO}_{4}$; while the majorities are solid under normal condition of temperature and pressure. The trait of transition metal oxides is the enormous range of electronic properties due to the progressive filling of shells of d-orbital across each series.(Cox, 1992) They can be good insulators (such as $\left.\mathrm{TiO}_{2}\right)$, metals $\left(\mathrm{ReO}_{3}\right)$, and even superconductors $\left(\mathrm{YBa}_{2} \mathrm{Cu}_{3} \mathrm{O}_{7}\right)$. Many of them show transitions from a metallic to an insulating state as a function of temperature $\left(\mathrm{VO}_{2}\right)$, pressure $\left(\mathrm{V}_{2} \mathrm{O}_{3}\right)$ or composition $\left(\mathrm{Na}_{x} \mathrm{WO}_{3}\right)$. With these variations in electrical conductivity, other physical properties related to the electronic structure become quite different, such as the optical and magnetic behaviors that form the basis of many important applications. On the other hand, these diverse electronic structures also left plenty mysteries for scientists to discover and brought many challenges to our understanding. 


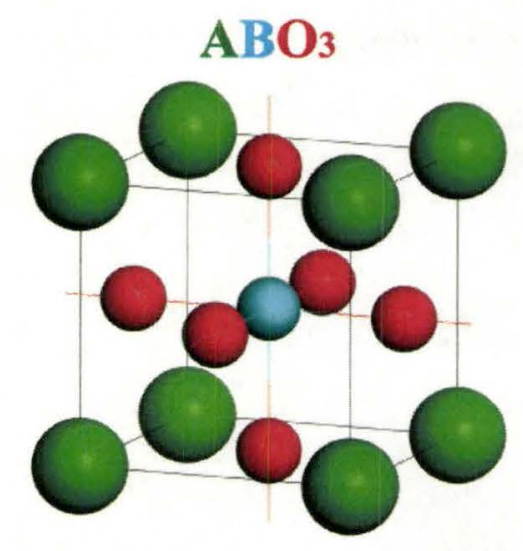

Figure 2.1: A model of an ideal cubic perovskite $\mathrm{ABO}_{3}$ unit cell

\subsection{Perovskite oxide and stability}

For the ternary oxides with the formula $\mathrm{ABO}_{3}$ where the $\mathrm{A}$-site cations are generally larger than the B-site cations and similar in size to the oxygen ions, their common structures are perovskite (Figure 2.1). In the ideal cubic perovskite unit cell, the A-site ion sits at the corner and the B-site ion sits at the body center surrounded by six face-centered oxygen ions. There are two cation sites and one anion site. With different ions' radius of A-site and B-site cations, different space groups can be achieved, resulting in a deviation from ideal cubic perovskite structure and even an amount of distortion. As a consequence the higher symmetry of crystal field is broken and the electronic structures relating to the local structure are modulated, generating all kinds of complex conducting behaviors.

The stability of the cubic perovskite structure is well estimated by the tolerance 
factor (Equation 2.1) introduced by Goldschmidt for the perovskite arrangement.

$$
t=\frac{R_{A}+R_{B}}{\sqrt{2}\left(R_{B}+R_{O}\right)}
$$

where the $R_{A}, R_{B}$ and $R_{O}$ are the average ionic radii for each lattice site. It predicts how far from ideal packing can the ionic sizes move and still be "tolerated" by the perovskite structure. A factor close to 1 foretells a cubic symmetry, and a factor away from 1 is less likely to be cubic. Ions on A or B-site can be partially substituted for different valent ions, hence enhancing or diminishing the density of charge carriers. The oxygen stoichiometry can also be affected by the extent of doping or the nature of the transition metal of B-sites.

By substituting cations on A or B-site, the structure distortion and electron doping can be modulated to some extent to control physical properties. Generally the larger cations go to the A-site and smaller cations go to the B-site. The ions substituted on A-sites commonly are main group elements, such as $\mathrm{Ca}, \mathrm{Sr}, \mathrm{Ba}$, and $\mathrm{Pb}$. For those ions that substitutes with the same valence of A-site ions, the substitutions only adjust the geometry of bonds and modify distortions; for those that substituted with the different valence of A-site ions, the substitutions function as releasing or retrieving electrons and their valence/conduction states usually are far away from Fermi level. The change in the electronic structure around Fermi level seems not to affect much the basis electron transportation network. For most A-site doping perovskite structure materials, the A-site cation ions don't contribute much to form the conducting network, and the disorder effect is not large. Therefore the distribution of substituted A-site ions in the lattice is generally not that crucial. For example, 
the physical properties of $(\mathrm{Ca}, \mathrm{Sr}) \mathrm{VO}_{3}$ (Inoue et al., 1995) and $(\mathrm{Ca}, \mathrm{Sr})_{2} \mathrm{RuO}_{4}(\mathrm{Lee}$ et al., 2002) are not affected by the A-site disorder.

However, an ion on B-site together with six-surrounded oxygen ions forming an octahedron plays a central role in the electronic structure by constituting the basis electron transportation network. The B-site substitution, especially for different valence state transitional elements, will disturb the band structure around Fermi level due to the non-negligible interaction between d-shell electrons. The basic transport network will change somehow due to the possible large disorder effect. The distribution of B-site ions will significantly affect the electronic transport properties. For example, the physical properties of $\mathrm{La}(\mathrm{Ni}, \mathrm{Mn}) \mathrm{O}_{3}$ and $\mathrm{La}(\mathrm{Ni}, \mathrm{Fe}) \mathrm{O}_{3}$ (Sarma et al., 1998) were explained in terms of electronic structural changes due to disorder.

\subsection{B-site substitution}

Since the B-site substitutions can significantly modulate the electronic structure therefore the physical properties of perovskites such as dielectric properties and resistivity, they are industrially important and must be elucidated. In fact the majority of the ordered perovskites studies are oxide perovskites on the B-site ordering. The most common B-site ordered perovskites have a formulae with $\mathrm{A}_{2} \mathrm{BB}^{\prime} \mathrm{O}_{6}$ or $\mathrm{A}_{3} \mathrm{BB}_{2}^{\prime} \mathrm{O}_{9}$ where $\mathrm{B}$ and $\mathrm{B}^{\prime}$ are different cations situating on distinct $\mathrm{B}$-sites: they are termed 1:1 or 1:2 ordering, respectively, and 1:3 ordering of B-site cations is very rare. When A-site ion is divalent, four combinations of $B$-site cations are possible for 1:1 B-site ordering: $\mathrm{A}^{2+}{ }_{2} \mathrm{~B}^{3+} \mathrm{B}^{5+} \mathrm{O}_{6} ; \mathrm{A}^{2+}{ }_{2} \mathrm{~B}^{4+} \mathrm{B}^{4+} \mathrm{O}_{6} ; \mathrm{A}^{2+}{ }_{2} \mathrm{~B}^{2+} \mathrm{B}^{6+} \mathrm{O}_{6}$; 
$\mathrm{A}^{2+}{ }_{2} \mathrm{~B}^{1+} \mathrm{B}^{7+} \mathrm{O}_{6}$. The ideal 1:1 ordered perovskites are ordered along (111) planes exhibiting complete long range order and no tilting of the $\mathrm{BO}_{6}$ or $\mathrm{B}^{\prime} \mathrm{O}_{6}$ polyhedral, which are usually called as "rock salt" structure. Besides "rock salt" structure,"layered" and "random" structures are also common to the 1:1 perovskites

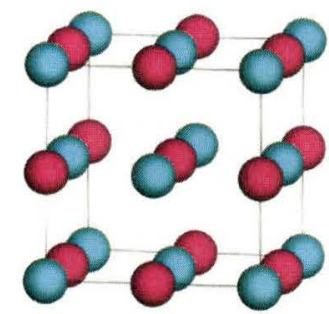

(a) Rock salt structure

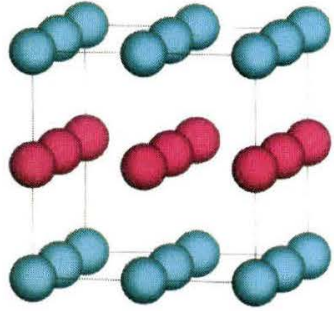

(b) Layered structure

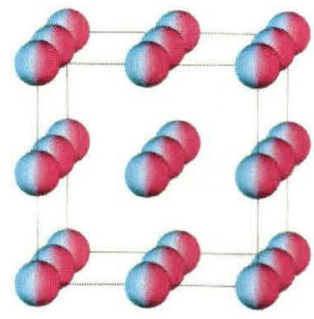

(c) Random structure

Figure 2.2: Three B-site sublattice types for 1:1 ordered perovskites

For the 1:1 B-site ordered perovskites with the rock salt or the layered structures, a superimposed Madelung energy (Equation 2.2) is introduced, which is the static Coulomb energy depending on the geometric arrangement of the constituent ions in the crystal structure. A charge or ion ordered structure may be induced by such a Madelung energy if it can significantly decrease the total energy.

$$
U_{M}=-\frac{z^{2} e^{2} M}{4 \pi \varepsilon_{0} r_{0}}
$$

where $z=$ charge of ions, $e=1.6022 \times 10^{-19} C, 4 \pi \varepsilon_{0}=1.112 \times 10^{-10} C^{2} /(\mathrm{Jm})$, and $M=$ Madelung contant. The Madelung constant is ions arrangement dependent (Equation 2.3)

$$
M=\sum_{i}( \pm)_{i} \frac{r_{0}}{r_{i}}
$$

where $r_{0}=$ distance to the closet ion. For rock salt structure perovskites, the super- 
imposed Madelung energy is basically the same as that for the ionic crystal $\mathrm{NaCl}$ (Equation 2.4)

$$
M=\sum_{i, j, k=-\infty}^{\infty} \frac{(-1)^{i+j+k}}{\left(i^{2}+j^{2}+k^{2}\right)^{1 / 2}}=1.74756 \ldots
$$

Hence the superimposed Madelung energy for the 1:1 B-site ordered perovskite is

$$
U_{M}=-1.74756 \times 331.984 \times 0.5 n^{2} / L \quad(k c a l / m o l e ~)
$$

where $n$ is the net charge away from the $4+$ average charge of B-site, $L$ is the lattice constant for ordered perovskite with unit of angstrom. From equation 2.5, one can see that the larger value of $\mathbf{n}$ the lower Madelung energy for a given lattice constant therefore the total lattice energy. The ordered arrangement is more favored for the larger charge difference on B-site. On the other hand the ordered arrangement will decrease the entropy against the total lattice energy. The two factors, Madelung energy and enthroy, will compete each other for an ordered arrangement even the dynamic effect not included. It is not surprising that order-disorder effects are most often encountered when the charge difference between $B$ and $B^{\prime}$ is two or less.

The discussion above is only focused on the ideal cubic 1:1 B-site ordered perovskite. However, the structure distortion will also affect the total lattice energy. Besides the most important factor, i.e. charge difference, three more factors such as ion radius difference for $\mathbf{B}$ and $\mathbf{B}^{\prime}$, cation coordination geometry and A-cation/Bcation size ratio also need to be considered, especially when the charge difference is two and B-site ion radius difference is less than 0.2. Figure 2.3 (Anderson et al., 1993) shows the three different B-site arrangements as charge difference and ionic 


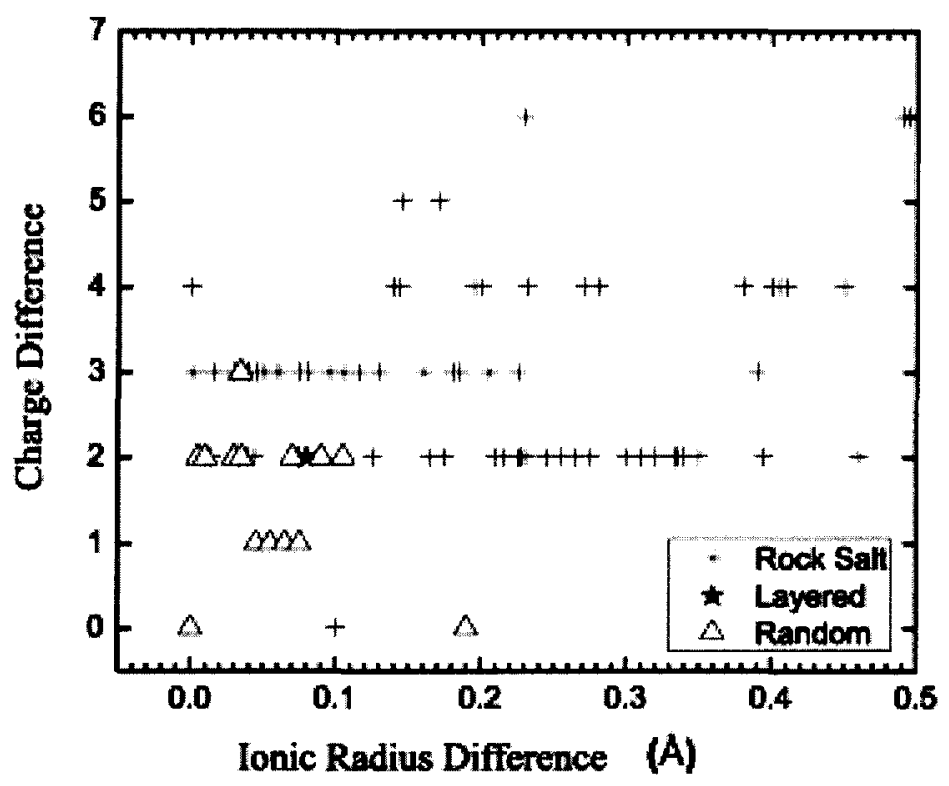

Figure 2.3: Structural dependence on charge Difference versus ionic radius difference (reproduced from Anderson et al. (1993))

radius change. It is clear that small charge and ionic radius difference lead to random arrangement while rock salt arrangement prevails at large charge and ionic radius difference. Table 2.1 is a summary of part 1:1 B-site ordered perovskites when charge difference is two (Anderson et al., 1993).

Structure distortion in perovskites results in deviation from ideal cubic structure, and the tilting of the $\mathrm{BO}_{6}$ octahedral structure usually results in different space group. A detailed space group determination can be achieved by X-ray and electron diffraction. A comprehensive structure description and determination of perovskites can be found in the book-Perovskites: modern and ancient (Mitchell, 2002). 


\subsection{Oxygen vacancies and doping effect}

The existance of oxygen vacancies during the growth condition usually cannot be neglected. In some semiconducting perovskites, such as $\mathrm{SrTiO}_{3}$ and $\mathrm{BaTiO}_{3}$, conductivity can change significantly due to existing oxygen vacancies. Even in some high temperature superconductors, oxygen vacancies are believed to play a crucial role. Generally oxygen vacancies result in n-type semiconducting perovskites and distort the local structure, causing a change of local electron density of states. Their ordering and segregation may cause an enhanced electron-phonon interaction, become defect recombination centers, or form a new electron transport network, resulting in a significant change in optical (Kan et al., 2005) and conducting (Poulsen et al., 1991) properties. Though oxygen vacancies are important to the electronic structure, it is difficult to control their content and distribution during experiments. Moreover, if a single crystal sample is required, it is not possible to control conductivity by oxygen vacancies.

Impurity doping is another way to control the conductivity. Impurity doping can increase the conductivity by several orders of magnitude. For the donor doping, additional electrons will prevent the formation of oxygen vacancies as described by the equation 2.6 using the Kröger-Vink notation.

$$
O_{o} \rightleftharpoons \frac{1}{2} O_{2}(g)+V_{o}+2 e^{-}
$$

The concentration of oxygen vacancies will significantly decrease and can be neglected to some extent at high donor content (Levis \& Catlow, 1986). Controlling 
the conductivity through doping is necessary in order to make highly conductive single crystal films for that are needed various electronic applications (Tomio et al., 1994).

\subsection{Metal-insulator transition}

The solids can be divided sharply into metals and insulator by defining a metal whose conductivity extrapolates to a finite value at zero temperature, and an insulator whose conductivity vanishes when extrapolated to zero temperature. Most undoped oxides are insulating, some show metallic behavior, and few are superconducting (Torrance et al., 1991). A few metal insulator transitions can be triggered by the change of temperature or the application of pressure, whereas more generally it is triggered by the change of stoichiometry or doping, which may dramatically change the conductivity. The phenomenon of Metal-insulator transition (MIT) is one of the fundamental problems in condensed matter physics and the obvious potential applications are as switches. Some explanations, such as band crossing, Mott transition and Anderson transition, have been developing in different systems for over 50 years.

\subsubsection{Band gap insulator}

It was quite unclear why electrons were free in metal and not free in insulator until the advent of quantum mechanics. Bloch energy bands theory, one of the earli- 
est successes of the quantum mechanics in solid, assumed periodic wave functions (Bloch wave functions) in periodic potential wells. The Bloch wave functions describe an electron with an infinite mean free path where scattering arises only when the lattice is not perfect due to impurity or thermal vibrations without considering electron-electron interaction. Bloch bands form by different quantum states with the similar energy, where electrons can occupy by obeying Fermi-Dirac statistic. The energy gaps between bands arise when there is no solution of the Schrödinger equation with the form of Bloch wave functions. If the Bloch energy bands are exactly filled, a finite energy gap will be generated between the ground state and all excited states, resulting in an insulting behavior; however, if the Bloch energy bands are partially filled, there is no energy gap resulting in a metallic behavior, which can be achieved when there is an odd number of electrons per unit cell or an even number of electrons per unit cell but Bloch bands are overlapping at the Fermi energy level. If two overlapping bands could be separated, for instance by pressure, temperature, or impurity doping through the change of ion spacing, a metal-insulator transition would occur. This explanation is valid for most main groups oxide, but it fails for many transition metal oxide where electron correlation effects are prominent.

\subsubsection{Mott-Hubbard gap}

Bloch energy band theory works well for most oxides, but encounters problems when oxides should be metals based on the Bloch energy bands theory but are insulators instead. From Hund's rule, a paired electrons would like to occupy an empty orbit with opposite spin configuration. However, the opposite spin configuration 
(usually called as low spin configuration) increases the possibility of interaction between the two electrons and gives rise to high Coulomb energy; while the parallel spin configuration (called as high spin configuration) has a lower Coulomb energy but would like to separate a pair of electrons into two orbits. By considering the electron-electron interaction, Mott proposed an explanation for those materials. In the simplest view, there is a competition between bandwidth $W$ and the intra-atomic Coulomb interaction $U$ that would like to keep a pair of electrons away from each other. The bandwidth $W$ is sensitive to pressure and represent the kinetic energy that can be gained by allowing charged excitons to propagate through the crystal; the intra-atomic Coulomb interaction $U$ opposes charge fluctuation and is not sensitive to pressure. When $U \leq W$, the material is a metal usually; when $U \geq W$, the repulsion will separate the Bloch band into two so-called "upper and lower" Hubbard bands, and the material shows insulating behavior. If the relationship between $U$ and $W$ can be reversed by pressure, temperature, impurity doping, a metal insulator transition would occur.

According to another helpful framework which also includes correlation effects has been introduced and developed by Zaanen, Sawatzky and Allen (ZSA) (Zaanen et al., 1985a; Zaanen \& Sawatzky, 1990), oxides can be described in terms of the relative energies of three electronic energy states near the Fermi level (Torrance et al., 1991) in figure 2.4. In each picture, the oxygen $2 p$-states are shown at the left and metal's states at the right. The metal's minimum unoccupied states lies at an energy $\Delta$ about the maximum occupied oxygen $2 p$-states. An energy $U^{\prime}$ separates the minimum unoccupied and maximum occupied states of metal. Here the prime on $U^{\prime}$ indicate the energy is not necessary to be Hubbard $U$. The bandwidth is 


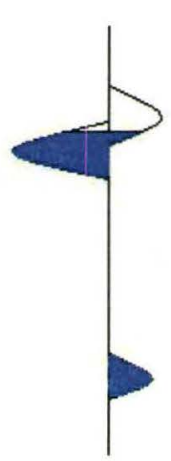

(a)

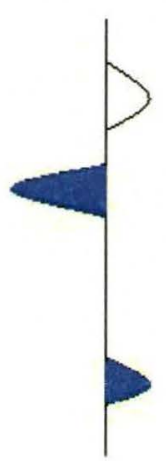

(b)

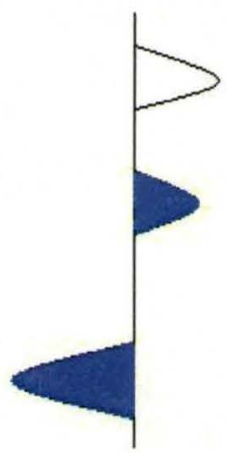

(c)

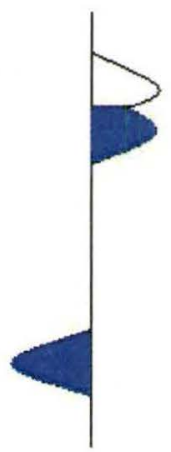

(d)

Figure 2.4: Schematic diagram of the relative energies of the oxygen and metal states in the ZSA framework (reproduced from Torrance et al. (1991)) with oxygen $2 p$-state is shown at the left and metal's states at the right. (a)Semi-metal (b) Charge transfer insulator (c) Mott-Hubbard insulator (d) Low $U$ metal. The energy values of $\Delta, U^{\prime}$ and $W$ are in the order of a few eV.

represented by $W$. Two different types of insulators and two different types of metals can be distinguished.

1. When $\Delta<W$ as figure $2.4(\mathrm{a})$, it is a metal and the lowest metal conduction band overlaps the oxygen $2 \mathrm{p}$-states.

2. When $W<\Delta<U^{\prime}$ as figure 2.4(b), it is a charge transfer insulator and the gap is dominated by $\Delta$.

3. When $W<U^{\prime}<\Delta$ as figure 2.4(c), it is a Mott-Hubbard insulator and the gap is dominated by $U^{\prime}$.

4. When $U^{\prime}<W$ as figure 2.4(d), it is a metal and the two metal bands overlaps to form a partially filled band. 


\subsubsection{Anderson localization}

In contrast to ordered systems, another explanation of metal insulator transition has been proposed for a system with random potentials (Anderson, 1958; Economou \& Cohen, 1970) that is usually treated as disorder effect by assuming a random spatial configuration of impurities. Although the impurities are located on regular lattice sites, the electron level on each site is different. That is considering a system of periodically arranged potential wells of varying depth. For ideal band structure without level disorder, the wave function will extend over the entire system and the intensity of a wave function initiated on one site will be zero after infinite time. However, if the strong disorder is present in the system, the true wave function will be localized and no spreading occurs at all. The neighboring sites can only feel some "tails" of the wave function with exponentially small amplitude, so that the intensity of the wave function remains finite at all time, resulting an insulating behavior (Shklovskii \& Efros, 1984).

According to Anderson's work, the conduction band in this kind of system has a range of so-called "localized states", which could trap electrons and would have a continuous range of energies. Even if their wave function will overlap, an electron cannot move from one of these local states to another without exchanging energy with lattice vibration. An energy called the "mobility edge $\left(E_{c}\right)$ " is used to separate these localized states from delocalized states. When the Fermi level lies above $E_{c}$, the material is a metal; when the Fermi level lies below $E_{c}$, the material is an insulator and charge transport occurs by hopping mechanism. If the sign of $E_{F}-E_{c}$ can be reversed by changing the number of electrons in the conduction band or the 
degree of disorder, a metal insulator transition would occur.

\subsubsection{Weak localization and conductance fluctuations}

Weak localization demonstrates as a positive correction to the resistivity of a metal or semiconductor which is not from the experimental error but a physical effect at low temperature in disordered electronic systems.

In disordered electronic systems, the electron motion does not occur along a straight line, but experiences a series of random scatterings off impurities resulting in a random "walk". The resistivity of a system is related to the probability of an electron to propagate between two given points in space. In classical physics, the total probability is the sum of all probabilities of the paths connecting the two points (equation 2.7), while in quantum mechanics the total probability is the probability of the sum of the quantum mechanical amplitudes of the paths (equation 2.8). Therefore the revised formula for the resistivity from a point A to point B includes the classical part plus a number of interference terms. The usual formula of the resistivity for a metal, the Drude model, corresponds to the former classical terms, and the weak localization correction corresponds to the latter quantum interference terms. The weak localization effect also called as quantum interference effect.

$$
\begin{gathered}
P=P_{1}+P_{2}=\left|A_{1}\right|^{2}+\left|A_{2}\right|^{2} \\
P=\left|A_{1}+A_{2}\right|^{2}=\left|A_{1}\right|^{2}+\left|A_{2}\right|^{2}+2\left|A_{1} A_{2}\right| \cos \theta
\end{gathered}
$$


In low-dimensional systems (films and wires), the trajectory of electrons scattering is confined. If the trajectory of two electrons is along a loop, the quantum phases cancel each other exactly and only left the quantum interference terms (Kramer \& Mackinnon, 1993), which make the weak localization effect more significant.

\subsection{Conduction mechanism}

There are many conduction mechanisms proposed for oxides due to the nature of their electronic structure, ranging from thermal excitation, hopping conduction to polaron conduction. In this section we will review these mechanisms briefly.

\subsubsection{Thermal excitation}

The most simple conduction mechanism could be thermal excitation. It happens in most band insulators and disordered insulators. For band insulators when the electron obtains sufficient thermal energy, it could overcome the band gap becoming a free electron (equation 2.9); For disordered insulators at high temperature, the resistivity is controlled by the process of electrons being excited to the mobility edge (Eqation 2.10) .

$$
\rho \propto \exp \left(\frac{E_{g}}{k T}\right)
$$


where $E_{g}$ is the energy of band gap.

$$
\rho \propto \exp \left(\frac{E_{c}-E_{F}}{k T}\right)
$$

where $\left(E_{c}-E_{F}\right)$ is the reduced band gap.

\subsubsection{Variable-range hopping}

Mott variable-range hopping process is one of hopping conduction mechanisms and usually applied to the disorder system. As to the the behavior of resistivity dependence on temperature for the case that $E_{F}$ lies below $E_{c}$, at low temperature the electron need to absorb as little energy as possible from phonons to jump to another state even if it is far away. The hopping probability at a given temperature depends on two parameters, $R$ the spatial separation of the sites, and $W$ their energy separation. This process could be regarded as hopping in a four-dimensional random array (three spatial coordinates and one energy coordinate) for 3-D hopping case, resulting a term of $T^{1 / 4}$. In general, for $d$-dimensions, this term becomes $T^{1 /(1+d)}$ where $d$ denotes the hopping dimension. This is observed in doped semiconductors at low temperature. The resistivity obeys the following relationship (Equation 2.11) (Mott, 1974).

$$
\rho \propto \exp \left(\frac{T_{M}}{T^{1+d}}\right)
$$

where $T_{M}$ is characteristic Mott temperature.

Another hopping mechanism is Efros-Shklovskii law, sometimes called E-S 
variable range hopping mechanism (E-S VRH), when electron correlation is prominent for disorder system at extremely low temperature (Shklovskii \& Efros, 1984). Due to the long-range Coulomb interactions, the soft Coulomb gap in the singleparticle density of states (DOS) of a system with interacting localized electrons vanishes at the Fermi level at low temperature when thermal excitations can not remove the gap. Applying the analytical expression of the single-particle DOS in the Mott derivation, a universal equation 2.12 would be obtained for any dimension.

$$
\rho \propto \exp \left(\frac{T_{E S}}{T^{1 / 2}}\right)
$$

where $T_{E S}$ is characteristic Efros-Shklovskii temperature.

For the aim of obtaining more insight into the nature of the conduction process at low as well as high temperature, a general form of a resistivity was assumed by Orgzall et al. (1996)

$$
\rho \propto T^{-b} \exp \left\{\left(\frac{T_{0}}{T}\right)^{p}\right\}
$$

where $b, T_{0}$, and $p$ are unknown but determinable parameters. The activation energy is

$$
\Delta E=\frac{d(\ln \rho)}{d(1 / k T)}=b k T+T_{0}^{p} p k T^{1-p} .
$$

The most important component $p$ is a characteristic quantity which describes the nature of hoping conduction mechanism. In the high temperature limit, the nearest neighbor hopping with $p=1$ is expected to be the dominant conduction process (Orgzall et al., 1996). With decreasing temperature the hopping changes to Mott variable range hopping by an exponent $p=1 /(d+1)$. At very low temperature the 
Coulomb interactions of the localized charge carriers have to be taken into account resulting in a change of the exponent $p=1 /(d+1)$ to $p=1 / 2$. However, the exponent $p=1 / 2$ might also come from a one-dimensional Mott variable range hoping process without a Coulomb gap in the density of states.

The determination of $p$ from experimental data is reliable if the temperature dependence of the resistivity is mainly determined by the exponential term in equation 2.13. This is guaranteed for strongly localized charge carriers, where the weak temperature dependence of the prefactor $T^{-b}$ can be neglected. If the charge carriers are weekly localized giving a small value of $T_{0}$ in equation 2.13 , the prefactor $T^{-b}$ cannot be neglected in the moderate- and high-temperature range. Only at very low temperatures is the exponential dependence in equation 2.13 expected to become the leading term.

\subsubsection{Polaron conduction}

Over the last 50 years, a variety of different theoretical models have been proposed to treat itinerant carriers interacting with a polarizable lattice. The concept of polarons is introduced to deal with electron-lattice interaction. A polaron is a quasiparticle consisted of an electron with surrounding polarization field. The polarization field will cause a local lattice distortion and the splitting of band structure near the Fermi level. As the polaron moves, the electron will carry the lattice distortion with it. Based on the degree of electron-lattice interaction, these models can be divided into two groups. The first (Pekar, 1946; Frohlich, 1954) treats the electron 
as a Bloch-wave-like delocalized particle called a Fröhlich or large polaron. The second (Holstein, 1959) assumes an extreme localization of the itinerant electron within one or several lattice sites called a small polaron accompanied by a strong local lattice deformation.

Besides single polaron, a bipolaron also can form when two like-signed charge carriers are bound within a common potential well produced by displacing atoms. If the two carriers are confined to a single basic structure unit, such as molecule, bond, or ion, the bipolaron is called small. Singlet small bipolarons are identified by the absence of spin. When dealing with semiclassical singlet small-bipolaron, Emin (1996) found that the most rapid hopping processes are a small-polaron hopping to a vacant site, and one of two carriers of a small bipolaron jumping onto the site of an adjacent small polaron. Consequentially, small polarons' hopping always dominates the DC resistivity even if most carriers form small bipolarons. The energy to break a small bipolaron into two small polarons contributes to the conductivity's activation energy.

The resistivity dominated by small polarons hopping process obeys the following law (Mott \& Davis, 1979) (equation 2.15) in the insulating range.

$$
\rho \propto T^{3 / 2} \exp \left(\frac{E}{k_{B} T}\right)
$$

However, in the metallic region, according to a theory of small-polaron conduction at low temperature (Lang \& Firsov, 1963; Bogomolov et al., 1968) (when 
$k_{B} T<2 t_{p}$ ), the resistivity can be expressed as equation 2.16 .

$$
\rho=\left(\hbar / n e^{2} a^{2} t_{p}\right)(1 / \tau)
$$

where $n$ is the charge carrier density, a is the lattice constant, $t_{p}$ is the hopping integral of polarons and $1 / \tau$ is the relaxation rate. As we know, the polaron is coming from the electron-phonon interaction, and phonon vibration modes usually contain two low frequency acoustic modes and one high frequency optical modes. However, only the optical mode will displace the charge center, cause a polarized electric field and then result a strong electron-phonon interaction. Therefore we only need to consider the interaction between the optical mode and the charge carries. Then the relaxation rate can be expressed as equation 2.17.

$$
1 / \tau=\sum_{a} A_{a} \omega_{a} / \sinh ^{2}\left(\hbar \omega_{a} / 2 k_{B} T\right)
$$

where $\omega_{a}$ is the average frequency of one optical phonon mode, $A_{a}$ is a constant depending on the bare conduction bandwidth and the electron-phonon coupling strength. As a consequence of the equation 2.17, only the low-lying optical modes with a strong electron-phonon coupling contribute to the resistivity at low temperature. Among the low-lying optical modes, only the softest optical phonon branch that is related to the tilting of the oxygen octahedral is strongly coupled to the carriers. However, the high frequency phonon modes such as the Jahn-Teller modes contribute little to the resistivity. Including the impurity scattering, the total resistivity can be expressed as equation 2.18 (Zhao et al., 2000).

$$
\rho=\rho_{0}+E W_{s} / \sinh ^{2}\left(\hbar \omega_{s} / 2 k_{B} T\right)
$$


where $E$ is a constant, being proportional to the effective mass of polarons, and $W_{s}$ is the average frequency of the softest optical mode.

Liu et al. (2005) provided evidence of the small polaron conduction mechanism in their single phase $\mathrm{BaTi}_{1-x} \mathrm{Nb}_{x} \mathrm{O}_{3}(0 \leq \mathrm{x} \leq 0.5)$ thin films. Kolodiazhnyi \& Wimbush (2006) found that dopant electrons formed immobilized small spin-singlet bipolarons in their $\mathrm{BaTi}_{1-x} \mathrm{Nb}_{x} \mathrm{O}_{3}$ ceramic samples $(0 \leq \mathrm{x} \leq 0.2)$ at low temperature, and the bipolarons gradually dissolved into two small polarons as the temperature increases. Since the small polarons' hopping always dominates the DC resistivity, the resisvity will decrease as more bipolarons dissolve into samll poarons at high temperature. 
Table 2.1: Examples of 1:1 B-Site ordered perovskites when charge difference is two (Anderson et al., 1993)

\begin{tabular}{clcl}
\hline \multicolumn{3}{c}{ Rock Salt } \\
\hline No & compound & $\Delta$ ion radius $(\AA)$ & comments \\
\hline 1. & $\mathrm{A}_{2} \mathrm{AlNbO}_{6}$ & 0.115 & $\mathrm{~A}=\mathrm{Ca}, \mathrm{Sr}$ \\
2. & $\mathrm{A}_{2} \mathrm{DyNbO}_{6}$ & 0.272 & $\mathrm{~A}=\mathrm{Ca}, \mathrm{Sr}, \mathrm{Ba}$ \\
3. & $\mathrm{A}_{2} \mathrm{ErNbO}_{6}$ & 0.250 & $\mathrm{~A}=\mathrm{Ca}, \mathrm{Sr}, \mathrm{Ba}$ \\
4. & $\mathrm{A}_{2} \mathrm{GdNbO}_{6}$ & 0.298 & $\mathrm{~A}=\mathrm{Ca}, \mathrm{Sr}$ \\
5. & $\mathrm{A}_{2} \mathrm{HoNbO}_{6}$ & 0.261 & $\mathrm{~A}=\mathrm{Ca}, \mathrm{Sr}$ \\
6. & $\mathrm{A}_{2} \mathrm{InNbO}_{6}$ & 0.160 & $\mathrm{~A}=\mathrm{Ca}, \mathrm{Sr}, \mathrm{Ba}$ \\
7. & $\mathrm{A}_{2} \mathrm{LuNbO}_{6}$ & 0.221 & $\mathrm{~A}=\mathrm{Sr}, \mathrm{Ba}$ \\
8. & $\mathrm{A}_{2} \mathrm{NdNbO}_{6}$ & 0.343 & $\mathrm{~A}=\mathrm{Ca}, \mathrm{Ba}$ \\
9. & $\mathrm{A}_{2} \mathrm{PrNbO}_{6}$ & 0.350 & $\mathrm{~A}=\mathrm{Ca}, \mathrm{Ba}$ \\
10. & $\mathrm{A}_{2} \mathrm{SmNbO}_{6}$ & 0.318 & $\mathrm{~A}=\mathrm{Ca}, \mathrm{Sr}, \mathrm{Ba}$ \\
11. & $\mathrm{A}_{2} \mathrm{TbNbO}_{6}$ & 0.283 & $\mathrm{~A}=\mathrm{Ca}, \mathrm{Sr}$ \\
12. & $\mathrm{A}_{2} \mathrm{YNbO}_{6}$ & 0.260 & $\mathrm{~A}=\mathrm{Ca}, \mathrm{Ba}$ \\
13. & $\mathrm{A}_{2} \mathrm{YbNbO}_{6}$ & 0.228 & $\mathrm{~A}=\mathrm{Ca}, \mathrm{Ba}$ \\
14. & $\mathrm{Ba}_{2} \mathrm{ScNbO}_{6}$ & 0.105 & \\
\hline
\end{tabular}

Layered

\begin{tabular}{clcl}
\hline No & compound & $\Delta$ ion radius $(\AA)$ & comments \\
\hline 1. & $\mathrm{La}_{2} \mathrm{CuSnO}_{6}$ & 0.080 & \\
\hline & & & \\
\multicolumn{4}{c}{ Random } \\
\hline No & compound & $\Delta$ ion radius $(\AA)$ & comments \\
\hline 1. & $\mathrm{Ba}_{2} \mathrm{CrBiO}_{6}$ & 0.155 & \\
2. & $\mathrm{Ba}_{2} \mathrm{FeTaO}_{6}$ & 0.005 & \\
3. & $\mathrm{Ba}_{2} \mathrm{InSbO}_{6}$ & 0.200 & \\
4. & $\mathrm{Pb}_{2} \mathrm{FeNbO}_{6}$ & 0.005 & \\
5. & $\mathrm{Pb}_{2} \mathrm{InNbO}_{6}$ & 0.160 & \\
6. & $\mathrm{Pb}_{2} \mathrm{SrNbO}_{6}$ & 0.105 & \\
7. & $\mathrm{Sr}_{2} \mathrm{CrMnO}_{6}$ & 0.005 & \\
8. & $\mathrm{Sr}_{2} \mathrm{FeVO}_{6}$ & 0.105 & \\
9. & $\mathrm{Sr}_{2} \mathrm{MnSbO}_{6}$ & 0.045 & \\
10. & $\mathrm{Sr}_{2} \mathrm{TaCrO}_{6}$ & 0.025 & \\
11. & $\mathrm{Sr}_{2} \mathrm{TaFeO}_{6}$ & 0.090 & \\
\hline
\end{tabular}




\subsection{Literature review of Nb-doped barium titanate}

$\mathrm{BaTiO}_{3}$ is one of the most well studied perovskite materials with applications and potential applications based on its wide ranging properties that include ferroelectricity, piezoelectricity and photorefractivity (Sayer \& Sreenivas, 1990; Cohen, 1992). In its stoichiometric form it is an insulator with a band gap of $3.2 \mathrm{eV}$. Significant effort, however, has gone into the production of conductive analogues to this material through the replacement of either trivalent ions on the $\mathrm{Ba}^{2+}$ site or pentavalent ions on the $\mathrm{Ti}^{4+}$ site (Chan et al., 1986; Gilbert et al., 1996). Among the potential candidates, the $\mathrm{Nb}^{5+}$ doped compound is particularly intriguing as it has the potential to emerge as an electrode material able to facilitate an epitaxial relationship with the technologically significant $\mathrm{BaTiO}_{3}$ and PZT/PT materials. Considerable effort has gone into the production of this conductive oxide in both the bulk polycrystalline (Wu \& Lin, 1994; Yoon et al., 2000; Yoon \& Kim, 2002; Kolodiazhnyi \& Wimbush, 2006) and thin film forms (Nagano et al., 1998; Gasparov et al., 2001; Lemée et al., 2002; Liu et al., 2005; Huang et al., 2006; Khan et al., 1999). The results obtained show wide discrepancies with regard to the transport properties as well as to the degree of niobium solubility within the $\mathrm{BaTiO}_{3}$ structure. Further interest in this material stems from reports of superconductivity (Gasparov et al., 2001) as well as magnetic anomalies attributed to spin-singlet small bipolarons(Kolodiazhnyi \& Wimbush, 2006). 


\subsubsection{Ceramic specimen}

Ceramic $\mathrm{BaTi}_{1-x} \mathrm{Nb}_{x} \mathrm{O}_{3}$ samples can been obtained by all kinds of methods, such as the top-seeded solution growth method (Chang et al., 1999), sol-gel methods (Maso et al., 2006), and most commonly used solid state reaction (Wu \& Lin, 1994; Yoon \& Kim, 2002; Kolodiazhnyi \& Wimbush, 2006). Second phase impurities were found in $\mathrm{BaTi}_{1-x} \mathrm{Nb}_{x} \mathrm{O}_{3}$ as $\mathrm{Ba}$ rich and Ti rich region and cation vacancies $\left(V_{B a}^{\prime \prime}\right.$ and $V_{T i}^{\prime \prime \prime \prime}$ ) were accumulated at grain boundary (Kutty et al., 1985; Koschek \& Kubalek, 1985; Chan et al., 1986; Jonker \& Havinga, 1982). A weak positive temperature coefficient of resistivity (PTCR) effect was also reported (Wu \& Lin, 1994; Miki et al., 1998; Yoon et al., 2000; Brzozowski \& Castro, 2004) which is a well-known characteristic of donor-doped polycrystalline $\mathrm{BaTiO}_{3}$ ceramic due to the difference of resistivity at grain boundary and in the bulk.

A convex inflexion in the resistivity curve was observed for most ceramic samples in the resistivity curve as $\mathrm{Nb}$ content increases. The insulating stoichiometric $\mathrm{BaTiO}_{3}$ became semiconductive up to a certain $\mathrm{Nb}$ content $(0.4-0.5 \mathrm{~mol} \%$ reported by Yoon \& Kim (2002) and $2.4 \mathrm{~mol} \%$ reported by Lemée et al. (2002)) and after that point became insulating again. One explanation by Wernicke (1978) related the critical point to the cation vacancy kinetics and the grain size. Generally, the grain sizes become less than $1 \mu \mathrm{m}$ beyond $0.4-0.5 \mathrm{~mol} \%$ donor content when the samples are sintered in air. The cation vacancy diffusion layer formed during the cooling process is not less than the grain size (Wernicke, 1978) in this case. As a consequence, the grain boundary contributions become dominant over the bulk properties, resulting in insulating behavior. Desu \& Payne (1990a,b) also explained 
this transition phenomenon by considering the grain boundary but in a different way. According to their study, an insulating behavior with higher doping concentration was caused by dopant segregation in the grain boundary region forming highly resistive layers. Another way to explain this behavior is the defect compensation mechanisms (Yoon \& Kim, 2002). As the donor concentration increases, the compensation mechanisms change from the electrons compensation mechanism which results in a semiconducting behavior, to the cation vacancies compensation mechanism which results in an insulating behaviors. To eliminate the contribution from grain boundaries, a series of coarse-grained specimens (grain sizes are about several tens of $\mu \mathrm{m}$ ) with different concentration was prepared (Yoon \& Kim, 2002) by adjusting the oxygen partial pressure during the sinter process. Based on charge neutrality condition and the defect reaction constants, several equations (Yoon \& Kim, 2002) were proposed to describe the effects of temperature, oxygen partial pressure, electron density, oxygen density and cation densities as a function of donor concentration. It was found that the critical donor concentration and oxygen vacancy concentration decreased with a decrease in temperature; the oxygen vacancy also decreases as the $\mathrm{Nb}$ concentration increases and can be neglect for high $\mathrm{Nb}$ concentration even with oxygen partial pressure as low as $P_{O_{2}}=10^{-8} \mathrm{~atm}$. A trend of the variations of each defect concentration with donor concentration was shown in figure 2.5. It was also noticed that when acceptors were added to donordoped $\mathrm{BaTiO}_{3}$ insulating sample in which the concentration of the donor is more than the critical composition, the sample could recover its semiconducting property (Peng \& Lu, 1988; Ting et al., 1990). This was due to the co-doping compensation effect, and the net donor concentration could be still below the critical concentra- 

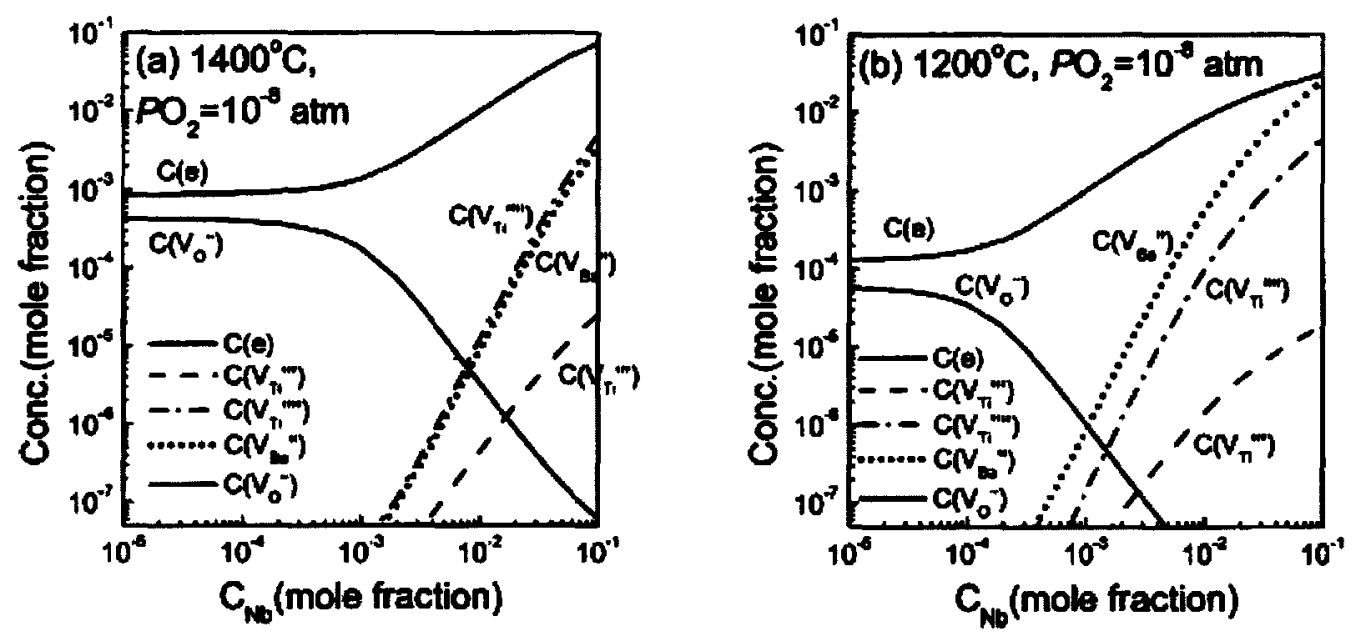

Figure 2.5: Calculated electron and defect concentrations at 1400 and $1200^{\circ} \mathrm{C}$ in a reducing condition v.s. the donor concentration (Yoon \& Kim, 2002)

tion. Although ceramic samples were synthesized by all different methods, the Nbdoping concentration was no more than 10 mol\% until Kolodiazhnyi \& Wimbush (2006) prepared a series of polycrystalline $\mathrm{BaTi}_{1-x} \mathrm{Nb}_{x} \mathrm{O}_{3}$ samples $(0 \leq \mathrm{x} \leq 0.2)$ by solid state reaction sintering in $3 \% \mathrm{H}_{2}+97 \% \mathrm{~N}_{2}$ gas flow to ensure electronic rather than cation vacancy compensation of the $\mathrm{Nb}$ dopant. In Kolodiazhnyi's study, no second phase was found and a bipolaron singlet forming at low temperature and gradually dissolving into two isolated small polarons was also proposed to explain an abnormally increased magnetic susceptibility around room temperature.

A electron paramagnetic resonance (EPR) study performed below $20 \mathrm{~K}$ for 100 ppm $\mathrm{Nb}$ concentration revealed that extrinsic ions $\mathrm{Nb}^{5+}$ was compensated by $\mathrm{Ti}^{3+}$ $\left(3 d^{1}\right)$ ions (Lenjer et al., 2002). The small $\mathrm{Ti}^{3+}$ polarons were stabilized by a tetragonal Jahn-Teller distortion. They claimed that although one would expect that a conduction electron had a lower energy at a $\mathrm{Nb}^{5+}$ site, one had to accept 
the experimental finding that the electron mainly resides at the Ti sites by the notation $\mathrm{Ti}^{3+}-\mathrm{Nb}^{5+}$. It was also noticed that many of $\mathrm{Ti}^{3+}$ ions were isolated and there was indirect evidence for the presence of bipolarons in the crystal ground state with a rather small dissociation energy $(0.01 \mathrm{eV})$ (Lenjer et al., 2002), which supported the conclusion of Kolodiazhnyi \& Wimbush (2006). Electron spin resonance (ESR) spectroscopy was also applied to detect impurity and intrinsic defects from 4.2-295 $\mathrm{K}$ in $\mathrm{BaTi}_{1-x} \mathrm{Nb}_{x} \mathrm{O}_{3}(0.1 \% \leq \mathrm{x} \leq 0.4 \%)$ single crystal as well as ceramics (Laguta2005). In this case, $\mathrm{Ti}^{3+}$ ions were also resolved but not with $\mathrm{Nb}^{5+/ 4+}$ impurities. Other minor intrinsic impurities also changed their valence state $\left(\mathrm{Cr}^{5+} \rightarrow \mathrm{Cr}^{3+}, \mathrm{Mn}^{4+} \rightarrow \mathrm{Mn}^{2+}\right)$ with $\mathrm{Nb}$ content increase due to the compensation of the excess charge of $\mathrm{Nb}^{5+}$ ions. The electrical conductivity below 300 $K$ was controlled by the electons localized on $\mathrm{Ti}^{4+}$ ions, forming polarons. This conclusion was valid for both single crystal and bulk in ceramics. A report on electron-phonon coupling in $0.9 \% \mathrm{Nb}$-doped $\mathrm{SrTiO}_{3}$ provided evidence for the existence of small polaron effect (Bi et al., 2006), but whether it came form local lattice distortion induced by $\mathrm{Nb}$ impurity size effect or the oxygen vacancy was not clear. The oxidation states of titanium and niobium were investigated in a relatively high $\mathrm{Nb}$ concentration compound $-\mathrm{Sn}_{x} \mathrm{NbTiP}_{3} \mathrm{O}_{12}(0 \leq \mathrm{x} \leq 0.50, \mathrm{Nb}: \mathrm{Ti}=1: 1)$ by X-ray photoelectron spectra (XPS) (Marco et al., 1997). The results showed a co-existence of $\mathrm{Ti}^{4+/ 3+}$ and $\mathrm{Nb}^{5+/ 4+}$ but no more than $75-80 \%$ of $\mathrm{Ti}^{4+}$ to $\mathrm{Ti}^{3+}$ or more than $20-25 \%$ of $\mathrm{Nb}^{5+}$ to $\mathrm{Nb}^{4+}$ could be achieved by fitting the data.

A superconducting behavior was found in a similar system ceramic $\mathrm{Nb}$-doped $\mathrm{SrTiO}_{3}$ with $T_{C}=370 m K$ (Pfeiffer \& Schooley, 1969). One end member, the ceramic non-stoichiometry $\mathrm{BaNbO}_{3-x}(0.6 \leq \mathrm{x} \leq 1)$ also exhibited superconductivity 
with $T_{C}$ as high as $22 K$ (Gasparov et al., 1994; Strukova et al., 1997) although the stoichiometric $\mathrm{BaNbO}_{3}$ was metallic but not superconducting (Casais et al., 1995). Single crystal $\mathrm{BaTi}_{1-x} \mathrm{Nb}_{x} \mathrm{O}_{3}(0.005 \leq x \leq 0.1)$ samples were also investigated and found to be semiconducting (Feltz \& Langbein, 1977). Optical and photorefractive properties of ceramic $\mathrm{BaTi}_{1-x} \mathrm{Nb}_{x} \mathrm{O}_{3}(x \leq 0.0005)$ (Chang et al., 1999) revealed that $\mathrm{Nb}$ firstly compensated for the residual p-type defects and gradually modified the photorefractive properties by changing charge carrier from holes $(x \leq 0.025 \%)$ to electrons $(x \geq 0.05 \%)$. The electronic and optical properties of the similar system $\mathrm{SrTi}_{1-x} \mathrm{Nb}_{x} \mathrm{O}_{3}(x=0.125,0.25,0.5)$ studied by ad initio method on assumed ordered structures showed that the Fermi level moved into the conduction bands with $x \geq 0.125$ and the valence band edge was distorted by $\mathrm{Nb}$ impurities (Guo et al., 2003).

\subsubsection{Thin films}

The ideal bulk single crystals growth of Nb-doped barium titanate was a difficult task and the heteroepitaxial growth of thin films had been viewed as an alternative method by which crystalline material in this system could be obtained.

Khan et al. (1999) successfully synthesized $\mathrm{BaTi}_{1-x} \mathrm{Nb}_{x} \mathrm{O}_{3}(0.01 \leq \mathrm{x} \leq 0.15)$ on $(00 \overline{0} 1)$ sapphire and $(001) \mathrm{SrTiO}_{3}$ substrate by laser ablation method at a substrate temperature of 700 and $650{ }^{\circ} \mathrm{C}$ respectively, under $200 \mathrm{mTorr}$ of oxygen and $\mathrm{N}_{2}$ atmosphere for the growth of and cubic phases. Rutherford backscattering spectrometry (RBS) results indicated a sharp interface between films and substrate. No 
$\mathrm{Nb}$ and $\mathrm{Ba}$ diffusion between films and substrate was detected within the resolution of RBS, and $\mathrm{Nb}$ really occupied $\mathrm{Ti}$ site. A variable-range hopping conduction mechanism was pointed out for $\mathrm{x}=0.02$ thin film sample by fitting resistance curve with $T^{-1 / 4}$ in the range of 150 to $300{ }^{\circ} \mathrm{C}$.

Semiconductive $\mathrm{BaTi}_{1-x} \mathrm{Nb}_{x} \mathrm{O}_{3}(0 \leq \mathrm{x} \leq 7 \%)$ were also grown by pulsed injection metal organic chemical vapor deposition method on $(012)_{h e x} \mathrm{LaAlO}_{3}$ substrates (Lemée et al., 2002). From room temperature up to $470 \mathrm{~K}$, no positive temperature coefficient behavior was observed while this effect had been reported in bulk samples. As the $\mathrm{Nb}$ concentration increases, an increase of the full width at half maximum (FWHM) of the (002) reflection of films indicates a loss of crystalline orientation perpendicular to the substrate's normal and films become polycrystalline. The decrease of the resistivity for $\mathrm{Nb}$ concentration less than $3 \mathrm{~mol} \%$ is due to the $\mathrm{Nb}$ ions being incorporated into lattice. Above that concentration, resistivity increases with the $\mathrm{Nb}$ concentration increases due to the formation of $\mathrm{Nb}_{2} \mathrm{O}_{5}$ phase.

In order to clarify whether the superconductivity found in $\mathrm{BaNbO}_{3-x}$ ceramics (Gasparov et al., 1994; Strukova et al., 1997) is an intrinsic property or due to other superconducting phase formed between grain boundary, thin films samples were studied. Thin films samples were grown on different substrates $\left(\mathrm{Al}_{2} \mathrm{O}_{3}\right.$, $\mathrm{NdGaO}_{3}, \mathrm{SrTiO}_{3}$ and $\mathrm{YSZ}$ ) by laser ablation methods instead of single crystals due to their high melting temperature $\left(T_{m}>1800^{\circ} \mathrm{C}\right)$. The $\mathrm{BaNbO}_{3-x}$ ceramic targets were grayish black in color rather than red as for stoichimometric $\mathrm{BaNbO}_{3}$, and the structures were cubic with lattice parameter of 4.32-4.35 $\AA$ which is somehow larger than that of stoichimometric $\mathrm{BaNbO}_{3}$ ceramics (4.09 $\AA$ ). Superconductiv- 
ity was found in $\mathrm{Ba}_{2} \mathrm{Nb}_{5} \mathrm{O}_{x}$ films with $T_{c}=14 \mathrm{~K}$ on $\mathrm{Al}_{2} \mathrm{O}_{3}$ but not in $\mathrm{BaNbO}_{3-x}$ films, confirmed by sheet resistance and AC susceptibility measurements. Possible explanation was relate to the very small variations in the oxygen composition.

Thin films of $\mathrm{BaTi}_{1-x} \mathrm{Nb}_{x} \mathrm{O}_{3}(0.01 \leq \mathrm{x} \leq 0.5)$ were also deposited on $\mathrm{MgO}(100)$ single crystal substrates by laser molecular beam epitaxy (L-MBE) under the oxygen pressure of $3.0 \times 10^{-4} \mathrm{~Pa}$ (Guo et al., 2004). In this work, the growth of $300 \mathrm{~nm}$ thick films was in situ controlled by reflection high energy electron diffraction (RHEED). From $\theta-2 \theta$ XRD scan, it was shown that no reflections from impurity phases or randomly orientated grains except $\mathrm{MgO}(l 00)$ and $\mathrm{BaTi}_{1-x} \mathrm{Nb}_{x} \mathrm{O}_{3}$ $(00 l)$. However, when $\mathrm{x}=0.5$, the (101) and (111) peaks were observed indicating the presence of a few other orientations in the thin films. Asymmetric 103 rocking curve XRD was applied to check the in-plane lattice parameters and the results were shown in figure 2.6. The lattice parameters $c, a$, and $V^{1 / 3}$ increased as $\mathrm{Nb}$ concentration increased, while $c / a$ decreased. Obviously, the thin films changed gradually from tetragonal to cubic structure with an increase of $\mathrm{Nb}$ concentration, and when $x \geq 0.2, c / a \approx 1$, the tetragonality almost vanished leading to a cubic structure. This structure change was also confirmed from Raman spectra with a characteristic tetragonal mode $A_{1}\left(\mathrm{LO}_{3}\right)$ around $727 \mathrm{~cm}^{-1}$ fading out when $\mathrm{Nb}$ concentration increased.

The same samples were used for resistivity measurement by Liu et al. (2005) from 77-600 $K$ using van der Pauw method. As Nb concentration increased, the resistivity dropped monotonously (Figure 2.7) in contrast to from others results (Yoon \& Kim, 2002; Nagano et al., 1998; Lemée et al., 2002). The temperature 


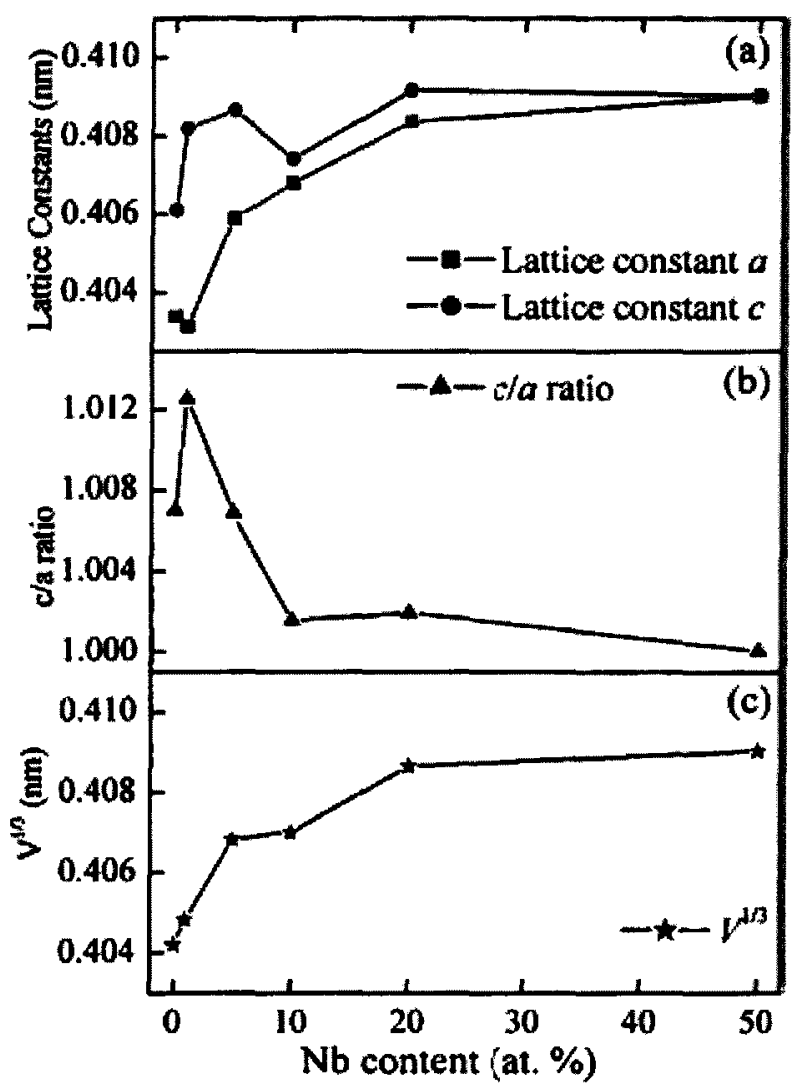

Figure 2.6: $\mathrm{BaTi}_{1-x} \mathrm{Nb}_{x} \mathrm{O}_{3}(00 l)$ thin films lattice parameters. (a) lattice parameter $c$ and $a$, (b) $c / a$ ratio, (c) unit cell volume $V^{1 / 3}$ (Guo et al., 2004)

dependence of the resistivity shown in figure 2.7 suggested a metal-insulator transition boundary around $x=0.2$. The Hall coefficients of all samples were negative, which indicated that major charge carries were electrons, and corresponding Hall mobility values were shown in table 2.2 . As one can see from table 2.2 , the carrier densities increased with an increase in $\mathrm{Nb}$ concentration, while the mobilities were not significantly different. The apparent different resistivity at room temperature were attributed to the the difference in the carrier density. Liu et al. (2005) also plotted the temperature dependence of the sesistivity and reciprocal of mobility for 
Table 2.2: Resistivity, carrier density, and Hall mobility of $\mathrm{BaTi}_{1-x} \mathrm{Nb}_{x} \mathrm{O}_{3}$ thin films at room temperature(Liu et al., 2005)

\begin{tabular}{llll}
\hline Samples & $\rho_{R T}(\Omega \cdot \mathrm{cm})$ & $\mathrm{n}\left(\mathrm{cm}^{-3}\right)$ & $\mu_{H}\left(\mathrm{~cm}^{2} / \mathrm{Vs}\right)$ \\
\hline $\mathrm{x}=0.01$ & 13.78 & $1.2 \times 10^{17}$ & 3.5 \\
$\mathrm{x}=0.05$ & 5.48 & $3.0 \times 10^{17}$ & 3.3 \\
$\mathrm{x}=0.1$ & 1.82 & $6.3 \times 10^{17}$ & 5.3 \\
$\mathrm{x}=0.2$ & $1.08 \times 10^{-2}$ & $6.4 \times 10^{19}$ & 9 \\
$\mathrm{x}=0.5$ & $2.70 \times 10^{-4}$ & $4.6 \times 10^{21}$ & 5.2 \\
\hline
\end{tabular}

$\mathrm{x}=0.5 \mathrm{BaTi}_{1-x} \mathrm{Nb}_{x} \mathrm{O}_{3}$ thin film (Figure 2.8), and claimed that the behavior of the temperature dependence of the resistivity highly depended on the mobility but not on the carries density. To explain conduction mechanism, Liu et al. (2005) used several models to fit their data, and supported the small polaron conduction scheme although the question of the nature of charge carriers in $\mathrm{BaTiO}_{3}$ is still open. The fitting results were shown in figure 2.9 .

- $\mathrm{x}<0.2$ semicondcuting thin films: polaron hopping mode

- $\mathrm{x}=0.2: 78-245 \mathrm{~K}$, small polaron metallic conduction mode; $>245 \mathrm{~K}$, thermal activation mode

- $\mathrm{x}=0.5$ : $<300 \mathrm{~K}$, small polaron metallic conduction mode; 300-500 K, conduction band electrons; $>522 \mathrm{~K}$ phonons scattering mode

Since the report of good conductivity in $\mathrm{BaTi}_{1-x} \mathrm{Nb}_{x} \mathrm{O}_{3}$ thin films (Liu et al., 2005), a $\mathrm{BaNb}_{0.3} \mathrm{Ti}_{0.7} \mathrm{O} 3 / \mathrm{Si}$ p-n junction was also fabricated by laser molecularbeam epitaxy (Huang et al., 2006), and demonstrated multifunctional properties of 


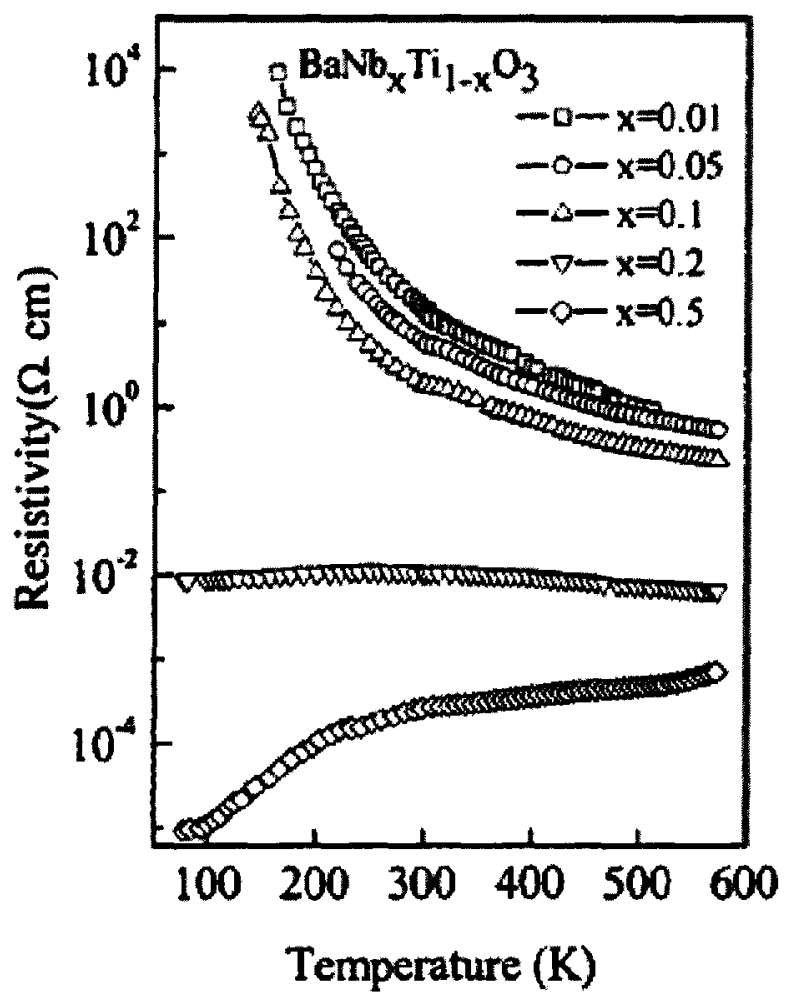

Figure 2.7: Temperature dependence of resistivity for $\mathrm{BaTi}_{1-x} \mathrm{Nb}_{x} \mathrm{O}_{3}(0.01 \leq \mathrm{x} \leq 0.05)$ thin films (Liu et al., 2005)

good rectifying $I-V$ characteristics, ultrafast photoelectric effects, and ferroelectric property.

In summary, there is no a full series study of $\mathrm{BaTi}_{1-x} \mathrm{Nb}_{x} \mathrm{O}_{3}$ with $x$ from 0 to 1. The early reports on bulk and thin film samples under higher oxygen partial pressure, had a limit $\mathrm{Nb}$ solubility $(<10 \%)$ in $\mathrm{BaTiO}_{3}$ lattice. The recent reports under lower oxygen partial pressure, had improved the $\mathrm{Nb}$ solubility up to $x=0.2$ for bulk sample (Kolodiazhnyi \& Wimbush, 2006) and up to $x=0.5$ for thin film sample (Liu et al., 2005). The different resistivity behaviors with an increase of $\mathrm{Nb}$ 


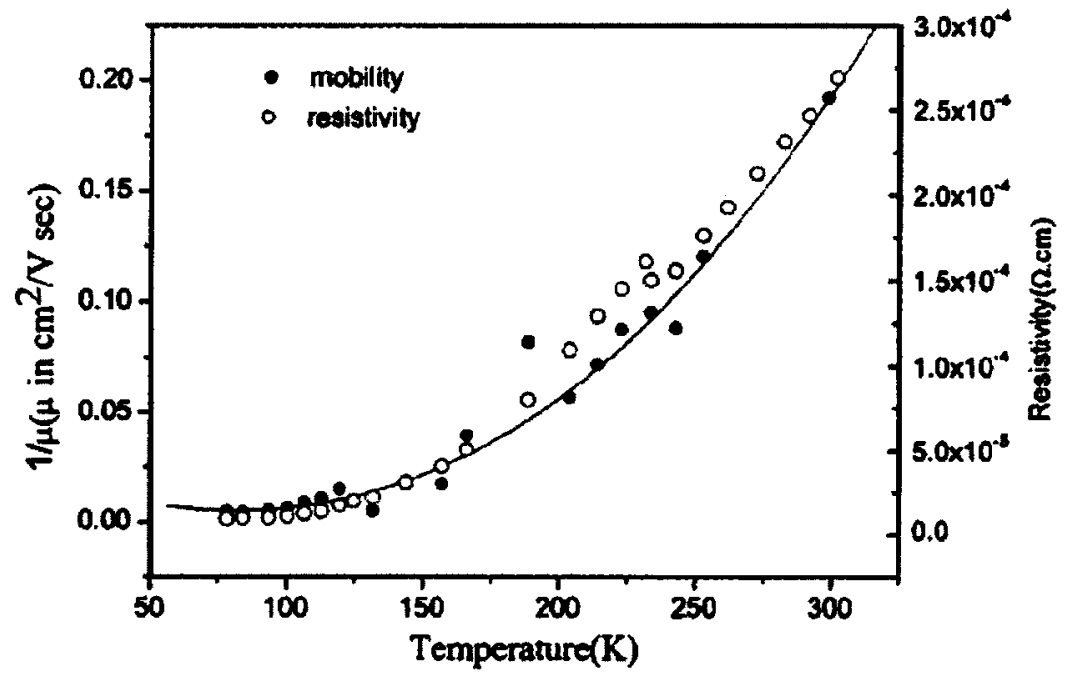

Figure 2.8: Temperature dependence of the resistivity and reciprocal of mobility of the thin films with $\mathrm{x}=0.5$ (Liu et al., 2005)
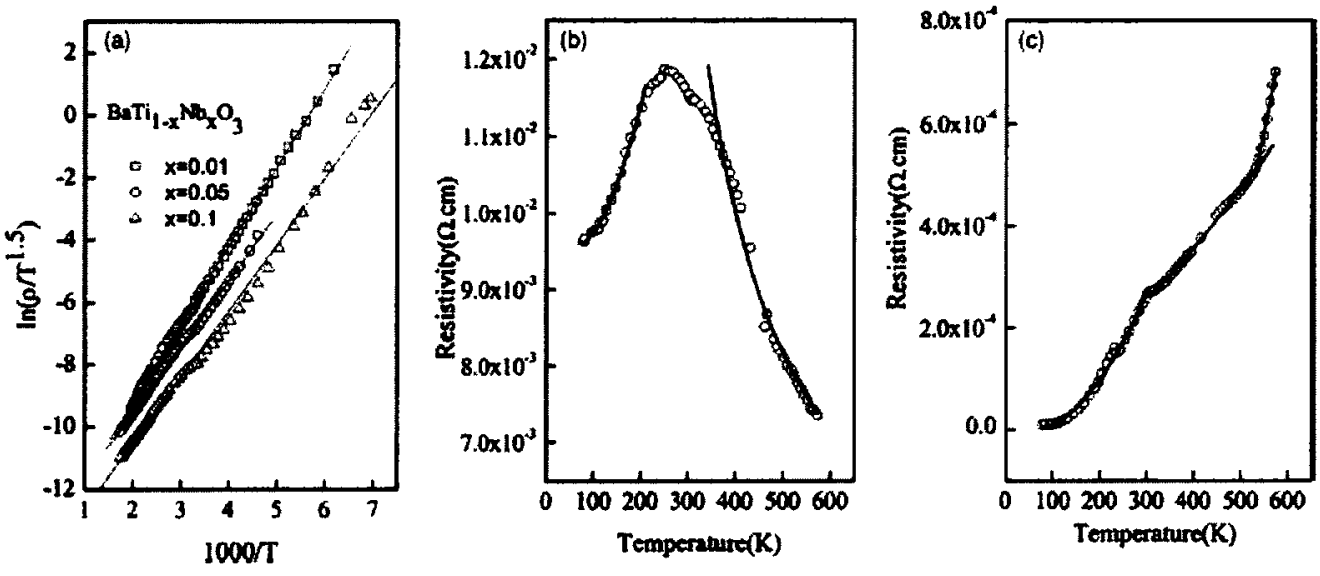

Figure 2.9: Conduction mechanism for different $\mathrm{Nb}$ concentration thin films. For all pictures, open dots are experimental data and solid lines are fitted curve (a) Arrhenius relations between $\ln \left(\rho / T^{1.5}\right)$ and $1 / T$ of thin films with $\mathrm{x}=0.01,0.05$ and 0.1 (b) Temperature dependence of resistivity of $\mathrm{x}=0.2$. The resistivity fitted to small polaron metallic conduction mode $\rho(T)=\rho_{0}+E \omega_{s} / 2 k_{B} T$ in the temperature interval $78-200 \mathrm{~K}$, and thermal activation mode $\rho=\rho_{0} e^{\Delta / k_{B} T}$ for the temperature higher than $300 \mathrm{~K}$ (c) For $\mathrm{x}=0.5$, the resistivity fitted to small polaron metallic conduction mode below $300 \mathrm{~K}, T^{1} .5$ in the interval 300-500 $\mathrm{K}$ and $T^{5}$ above $522 \mathrm{~K}$ conduction mode (Liu et al., 2005) 
concentration by different synthesis method ranging from ceramics to thin films, involved the use of different oxygen partial pressure. The better conductivity of samples seems to need to control or optimize the oxygen partial pressure, and changes the compensation mechanism from one that involves cation vacancies to the one that mainly involves electrons. To keep the samples in an electron conduction scenario, the low oxygen partial pressure is necessary. 


\section{Chapter 3}

\section{Characterization Techniques}

In order to obtain structure information of thin films, several characterization techniques are used. Powder X-ray diffraction (XRD) is used to identify the phase purity and lattice parameter normal to the substrate surface. 2D-XRD is used to generate pole figures illustrating the grain orientations. Scanning electron microscopy (SEM) can provide the rough surface morphology of the films, and atomic force microscopy (AFM) can provide more detail surface roughness of the substrate. Transmission electron microscopy (TEM) together with X-ray energy dispersive spectrometer (XEDS or EDS) and energy loss spectroscopy (EELS) provide a wealth of information about grain sizes, superlattice structure, heterogeneous interface, element composition and also the valence state. Four probe van der Pauw method was used for resistivity measurement. The Hall coefficients were measured by the ACCENT, HL5500 PC Hall Effect Measurement System, and magnetic properties were measured by physical properties measurement system (PPMS) 


\subsection{X-ray diffraction}

$\mathrm{X}$-ray diffraction (XRD) is one of non-destructive analytical techniques that is used to measure the atomic arrangement of materials. As a monochromatic X-ray beam hits a sample, in addition to absorption and other phenomena, the X-ray scattering with the same wavelength as the incident beam can be observed, called coherent $\mathrm{X}$-rays scattering. The coherent scattering of X-ray from a sample is a function of the electron distribution in the sample. There are many theories and equations to describe the relationship between the diffraction pattern and the material structure. Among them, Bragg's law is a simple relationship linking the diffraction angle to the spacing between crystalline planes (equation 3.1).

$$
\lambda=2 d \sin \theta
$$

where $\lambda$ is the wavelength, $d$ is the distance between two adjacent crystal plane ( $d$-spacing), and $\theta$ is the Bragg angle at which one observes a diffraction peak.

\subsubsection{Traditional powder X-ray diffraction}

Figure 3.1 show a typical diffraction pattern in 3D space with a point or positionsensitive detectors which have to be fixed on the detection circle. This is the traditional X-ray powder diffraction configuration. With such configuration, only the diffraction peaks on the detection circle can be recorded. 


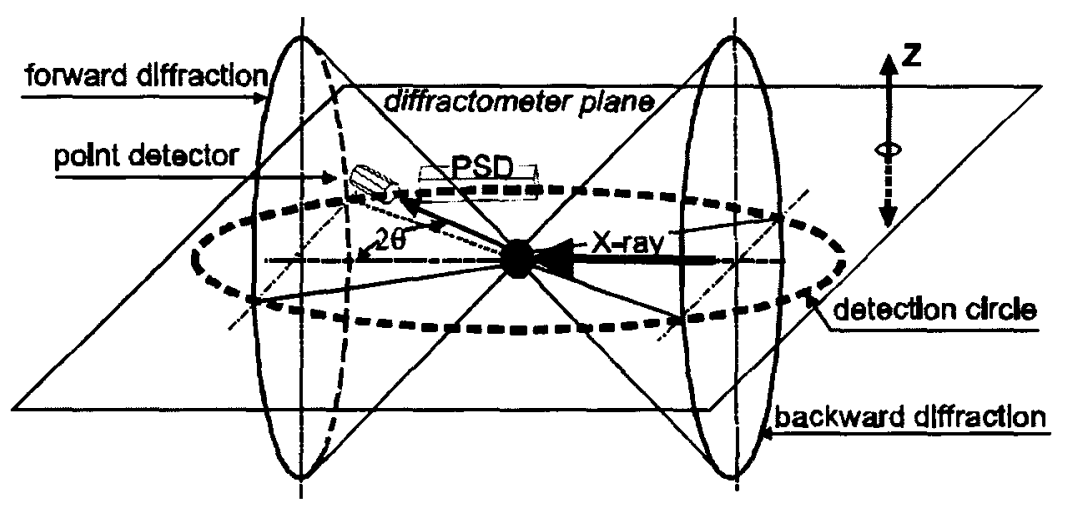

Figure 3.1: Diffraction patterns in 3D space from a powder sample and the diffractometer plane. (GADDS4.0, 1999)

\subsubsection{Two-dimensional X-ray diffraction}

Two-dimensional X-ray diffraction (2D XRD or $\mathrm{XRD}^{2}$ ) is a new technique in the field of X-ray diffraction, which is not simply equipped with a two-dimensional detector, but also involves 2D image processing and 2D diffraction pattern manipulation and interpretation. Figure 3.2 shows the system configuration. Two coordination systems, i.e. the laboratory system and the sample system, were used in different circumstances to simplify the expression. Their relationship is illustrated in figure 3.3. In figure 3.4, it shows the detector position in the laboratory system and how the diffraction cones are recorded. All diffraction rings are collected by an appropriate scan scheme of $\phi, \omega$ or their combination.

With this technique, it is possible to retrieve a standard "powder pattern" $(\theta-2 \theta$ scan) if integrating an area detector frame in the $\chi$ direction; and a pole figure if 


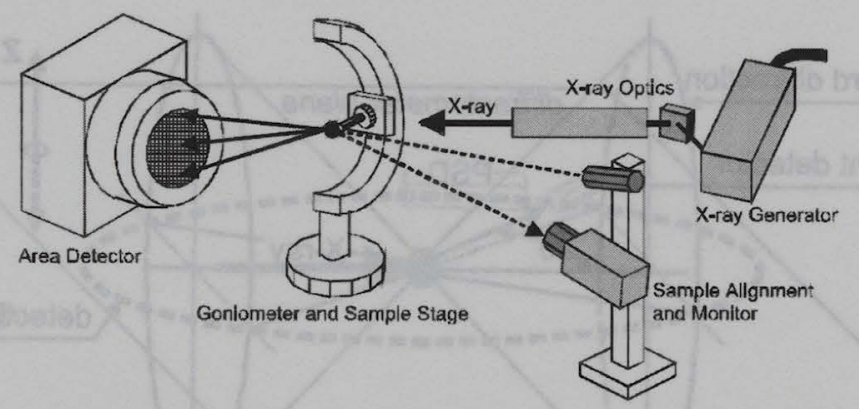

Figure 3.2: Five major units in a GADDS system: X-ray generator (sealed tube); X-ray optics (monochromator and collimator); goniometer and sample stage; sample alignment and monitor (laser-video); and area detector. (GADDS4.0, 1999)

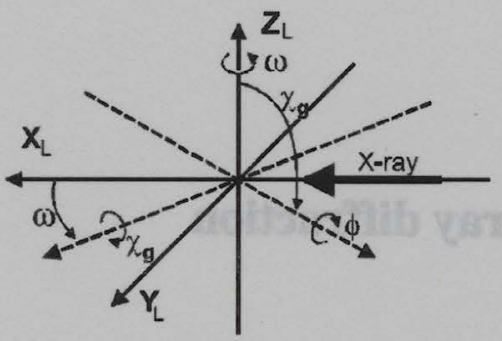

(a)

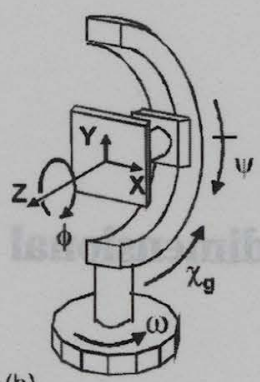

(b)

Figure 3.3: Sample rotation and translation in the laboratory system. (a) Relationship between rotation axes and $\mathrm{X}_{L} \mathrm{Y}_{L} \mathrm{Z}_{L}$ coordinates; (b) Relationship among rotation axes $\left(\omega, \chi_{g}, \psi, \phi\right)$ and translation axes XYZ. (GADDS4.0, 1999)

an appropriate $2 \theta$ range is integrated with the help of the GADDS (General Area Detector Diffraction System) software. Hence phase ID, texture, residual stress, and percent crystallinity can be obtained.

\subsubsection{Asymmetric rocking curve X-ray diffraction}

Usually the rocking curve X-ray diffraction is used to check the quality of films by measuring the peak's FWHM of the out-of-plane reflections, while the asymmetric 


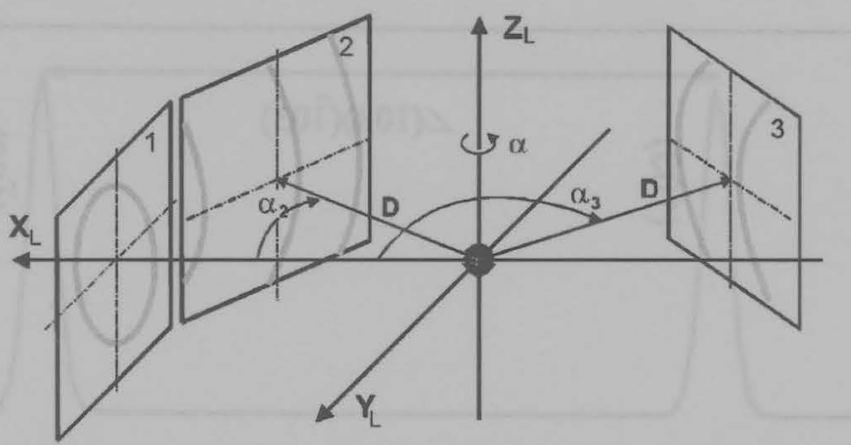

Figure 3.4: Detector position in the laboratory system $X_{L} Y_{L} Z_{L}$. D is the sample to detector distance; $\alpha$ is the swing angle of the detector. (GADDS4.0, 1999)

rocking curve X-ray diffraction is used to resolve in-plane lattice parameters by measuring the angle between two non-parallel planes having the same $d$-spacing. The angle of X-ray source and detector is fixed at the exact Bragg angle of the planes and move together on the $\omega$ track as $\omega-\theta$ scan. Typically the result contains two sharp peaks (figure 3.5), and the angle between such two planes can be read out directly. Thus the ratio of $a / c$ (or $b / c$ ) can be calculated from equation 3.3. By combining these results with powder X-ray diffraction result which giving the outof-plane lattice parameter $c$, the in-plane lattice parameters can be resolved. 


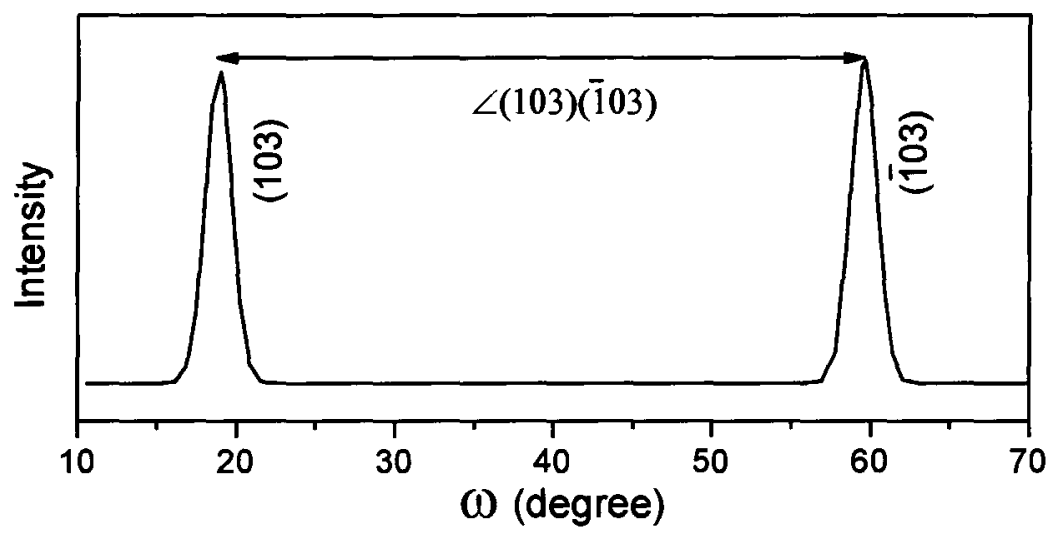

Figure 3.5: Typical asymmetric rocking curve results (reproduced from Guo et al. (2004)).

$$
\begin{aligned}
(k 0 l) \cdot(\bar{k} 0 l) & =|k 0 l| \cdot|\bar{k} 0 l| \cdot \cos \theta \\
a^{2} l^{2}-c^{2} k^{2} & =\left(a^{2} l^{2}+c^{2} k^{2}\right) \cdot \cos \theta \\
\frac{c}{a} & =\frac{l}{k} \sqrt{\frac{1-\cos \theta}{1+\cos \theta}}=\frac{l}{k} \tan \left(\frac{\theta}{2}\right)
\end{aligned}
$$

\subsection{Electron microscopy}

Conventional transmission electron microscopy (TEM), Philips CM12, was used to check our films' quality in imaging mode, $\mathrm{Nb}$ ions distribution in diffraction mode, and possible local phase segregations by X-ray energy dispersive spectrometer (XEDS or EDS). FEI Titan 80-300 Cubed equipped with a CEOS-designed hexapole-based aberration corrector for the image-forming lens and one for the probe-forming lens was used to obtain lattice images to characterize the heteroge- 
neous interface between films and substrate, and possible defects. Electron energy loss spectroscopy (EELS) with a monochromator integrated in the FEI Titan 80-300 was used to understand material's local electronic structure.

\subsection{Electron energy loss spectroscopy}

The Electron energy loss spectroscopy (EELS) in the TEM involves the measurement of the energy injected to a thin specimen $(<100 \mathrm{~nm})$ by fast incident electron $(>100 \mathrm{KeV})$. A typical EELS spectrum (figure 3.6) can be separated into three parts: (1) an sharp and intense zero loss peak (ZLP), where electrons pass through the specimen without any energy loss, representing an experimental energy resolution by its full width at half maximum (FWHM); (2) a series of low energy loss peaks $(<50 \mathrm{eV})$, so-called low loss peaks, involve the energy transition between intra-band and inter-band; (3) several low intensity but distinct high energy loss peaks, so-called core loss peaks, identify the presence of specific elements. The modulations over the core loss are known as the energy loss near edge structure (ELNES). The ELNES is due to a particular energy loss of incident electrons by exciting core electrons to the unoccupied states. The unoccupied states are related to the details of the local atomic environment, such as valence, type of bonding and coordination. Therefore the ELNES can be used to measure the local electronic structure in materials with sub-nanometer spatial resolution.

To extract and also understand the information behind ELNES, physical mod- 


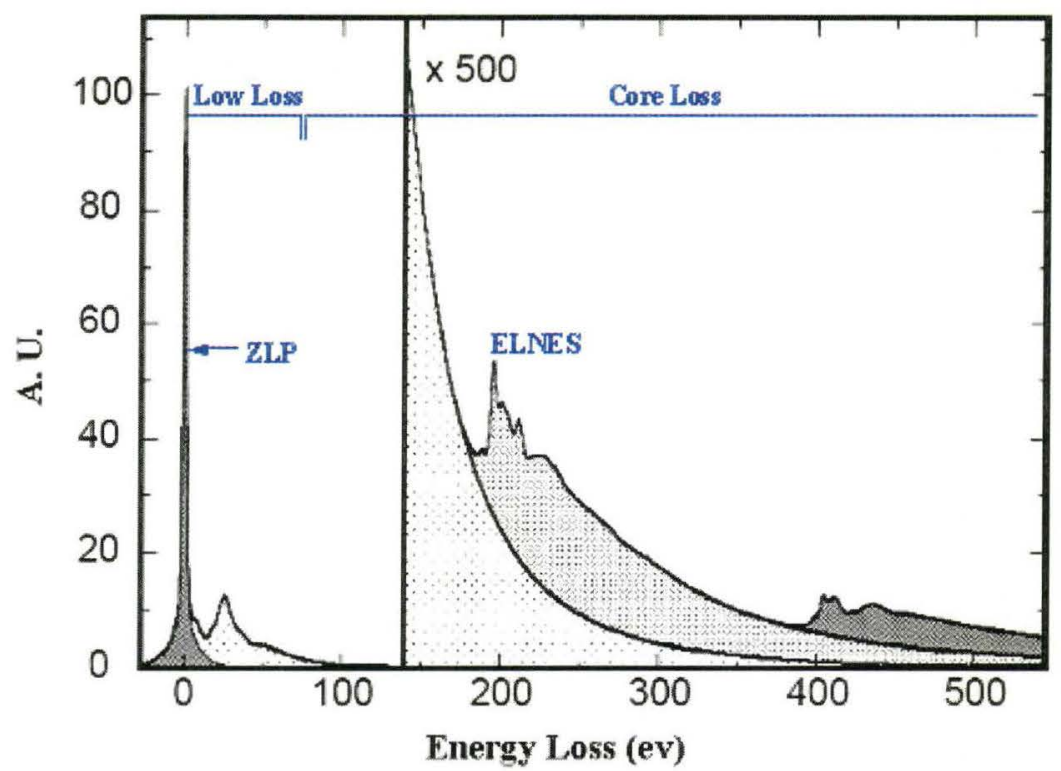

Figure 3.6: A schematic representation of a typical electron energy loss spectrum, (EELS Imaging and Analysis School, Gatan (2000))

els and mathematic descriptions were constructed. First of all, two simplifications known as the Born-Oppenheimer approximation and Franck-Condon principle can be made due to the relatively fast excitation timescale $\left(10^{-19}-10^{-20} \mathrm{~s}\right.$ for $\mathrm{O}-\mathrm{K}$ shell at $200 \mathrm{KeV})$ to the atom vibrational timescale $\left(\sim 10^{-13} \mathrm{~s}\right)$. As the consequence, the available electronic energy levels for the excited electron are simply those of the fixed atomic geometry with a constant distance. These approximations make the interpretation and the simulation of ELNES simple. The electron states in materials can be described by a set of wave functions, $\psi(r)$, which are obtained by solving the Schrödinger equation for the potential in the solid. The ejected electron makes a transition between two states. Assuming that the other electrons in the atom are not affected by the excitation process (this is known as the one-electron approximation), the available final states for the excited electron are the unoccupied 
states. Thus the effect of the core-hole is explicitly neglected in this particular case, but the core-hole effect can be considered with other approximations. The probability of the transition, and hence the intensity of the transition, from an initial state $\psi_{i}(r)$ (core states) to a final state $\psi_{f}(r)$ (originally, unoccupied states) at a given energy loss and scattering angle $I(E, \theta)$ obeys the Fermi Golden Rule (equation 3.3).

$$
I(E, \theta) \propto \frac{4 \gamma^{2}}{a_{0}^{2} q^{4}}\left|\int \psi_{i} \exp (i q \cdot r) \psi_{f}^{*} d^{3} r\right|^{2} \cdot \rho(E)
$$

where $a_{0}$ is the Bohr radius $(0.053 \mathrm{~nm}), \rho(E)$ is a quantity known as the density of states (DOS), and $q$ is the momentum transfer to the specimen. For a small angle scattering, $q$ can be described as equation 3.4

$$
q^{2}=k_{0}^{2}\left(\theta^{2}+\theta_{E}^{2}\right)
$$

where $k_{0}$ is the wave vector of incident beam, $\theta$ is the collecting angle, and $\theta(E)$ is the most probable scattering semiangle for an energy loss $E$.

In TEM, the energy loss is always much smaller than the energy of the incident beam, leading to a small momentum transfer $q$ relative to the electrons total momentum. Moreover, a collection aperture usually is used in front of the energy loss spectrometer to limit the maximum collecting angle and as a result the momentum transfer $q$. The dipole approximation is valid under most experimental condition by assuming a small $q$, and the $\exp (i q \cdot r)$ term in equation 3.3 only needs to be expanded to first order. The intensity of a transition then is rewritten as equation 3.5.

$$
I(E, \theta) \propto \frac{4 \gamma^{2}}{a_{0}^{2} q^{4}}\left|\int \psi_{i}(q \cdot r) \psi_{f}^{*} d^{3} r\right|^{2} \cdot \rho(E)
$$


There are two terms in equation 3.5. The integral part, or transition matrix term, represents the radial overlap of the initial and final state wave function, giving the basic edge shape, such as sawtooth shape for $\mathrm{K}$ edges and a more rounded shape for L edges. The density of states (DOS) term, $\rho(E)$, dominates the fluctuation superimposed on the basic edge shape. Therefore the ELNES simply reflects the energy distribution of the unoccupied electronic states.

Generally the transition matrix term is non-zero under some restrictions. According to the dipole selection rule, only when the change in the angular momentum $\Delta l= \pm 1$, can a transition occur, which means that the ELNES measures only a subset of the total DOS with an appropriate angular momentum symmetry. Secondly, the initial state is a core state, localized on a particular atom, and therefore the final state must have a component localized on the same atomic site. For these reasons, the ELNES is said to measure a site- and symmetry-projected DOS. More detail work about EELS process and application can be found in Egerton's book (Egerton, 1996) and a review paper (Keast et al., 2001).

\subsection{The van der Pauw method}

The Van der Pauw method is one of the most commonly used methods for doing four-probe resistivity measurement regardless of the shape of the sample. It provides an easy way to calculate sheet resistance, if the thickness of the sample is known, and therefore the resistivity. 


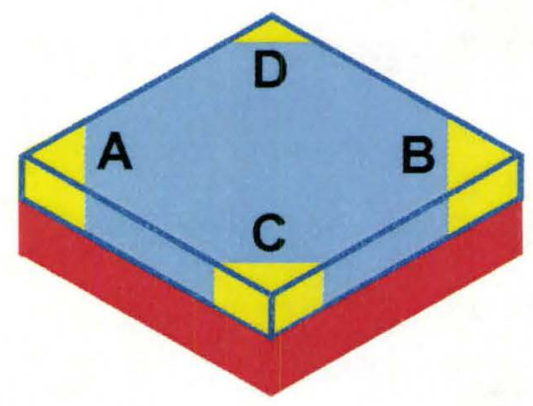

Figure 3.7: Contact placements for Hall and resistivity measurement

In order to use this method, the thickness of the sample must less than the width and length of the sample, and the sample must be homogeneous. The ohmic contacts must be small and placed on the boundary of the sample as possible as it can. One of the possible contact placements is shown in figure 3.7 The positive DC current $I_{A B}$ is injected into contact $A$ and taken out of contact $B$, and the DC voltage $V_{C D}$ is measured between contacts $C$ and $D$ with no externally applied magnetic field. Then a resistance can be calculated by Ohm's Law (Equation 3.6)

$$
R_{A B, C D}=\frac{V_{C D}}{I_{A B}}
$$

Based on the reciprocity theorem, $R_{A B, C D}=R_{C D, A B}=R_{B A, D C}=R_{D C, B A}$, and $R_{B C, D A}=R_{D A, B C}=R_{B A, D C}=R_{D C, B A}$. To do a more accurate measurement, one can define a horizontal and vertical resistance as equation 3.7 and3.8.

$$
R_{\text {horizontal }}=\left(R_{A B, C D}+R_{C D, A B}+R_{B A, D C}+R_{D C, B A}\right) / 4
$$




$$
R_{\text {vertical }}=\left(R_{B C, D A}+R_{D A, B C}+R_{C B, A D}+R_{A D, C B}\right) / 4
$$

The sheet resistivity $R_{s}$ can be obtained by solving the relationship (Equation 3.9), which was proposed by (van der Pauw, 1958).

$$
e^{-\pi R_{\text {horizontal }} / R_{s}}+e^{-\pi R_{\text {vertical }} / R_{s}}=1
$$

Each of our $\mathrm{BaTi}_{1-x} \mathrm{Nb}_{x} \mathrm{O}_{3}$ thin films for resistivity measurement was made by first placing four gold contacts on top of the corner of the bare $\mathrm{MgAl}_{2} \mathrm{O}_{4}$ substrate and then depositing the $\mathrm{BaTi}_{1-x} \mathrm{Nb}_{x} \mathrm{O}_{3}$ layer. $\mathrm{A}$ thin layer of $\mathrm{MgAl}_{2} \mathrm{O}_{4}$ about few $\mathrm{nm}$ thick was put on top of each sample as a capping layer. The gold contacts allowed for resistivity measurements as they protruded through both layers. The dimension of each thin film was $7 \times 7 \mathrm{~mm}$.

The Hall measurement in the van der Pauw technique can be used to determine the carrier density $n$ with known thickness $(d)$ of the sample by measuring the Hall voltage $V_{H}$. The Hall voltage is measured with a constant current $I$ and a constant magnetic field $B$ which is perpendicular to the surface of the sample. The current $I$ is go through a pair of contacts A and B as illustrated in figure 3.7, and the Hall voltage $V_{H}$ is measured across the remaining pair of contacts $\mathrm{C}$ and $\mathrm{D}$. Therefore the sheet Hall coefficient $R_{H}$ and carrier density $n$ can be calculated by equation 3.10 with known values of $I, V_{H}, B$ and $d$.

$$
R_{H}=\frac{V_{H}}{I B}=-\frac{1}{n d e}
$$




\section{Chapter 4}

\section{Electronic Structure Calculation}

\section{Techniques}

In order to properly understand the transport properties, electronic structure calculations were conducted. The calculations are not only use to reveal the materials' physical properties but also to potentially help in designing materials with improved properties. There are electronic structure calculation methods suited for different length scale, from single atoms including crystal fields (so called multiplet calculations), small clusters (molecular orbital calculations) and large clusters (with multiple scattering methods) to periodic structures (using band structure approaches). Each method has its advantages and disadvantages. For our study purpose, we choose band structure approach (WIEN2k program) because more comprehensive electronic structure information, such as partial density of states, electron density distribution and easy calculations of EELS spectra (include the O- $K$ edge), can be 
obtained within one program. We also used the multiplet method to obtain the more accurate $L$ edge information of transitional metals since these edge cannot be calculated with the band structure techniques. .

\subsection{WIEN2k}

WIEN2k is one of the most frequently used first-principles programs based on the band structure approach. The "first principle" means all calculations only depend on the nature and position of atoms without input of any experimental parameters. Based on the full-potential (linearized) augmented plane wave (LAPW) + local orbitals (lo) methods, under the frame of density functional theory (DFT) with local density approximation (LDA) or the improved version of generalized gradient approximation (GGA), electronic structure calculations are carried out by the program package WIEN2k . It is an all-electron scheme including relativistic effects. Band structure, density of states (DOS) and many other properties can be readily reproduced. The shortcomings are that the band gap is always underestimated, and as to the ELNES calculation, they are successful for $K$-edges but not for $L$ - and $M$-edge. The basic ideas behind WIEN2k program will be sketched out as following.

A solid consists of heavy positively charged nuclei, and lighter, negatively electrons. If one sample have $N$ nuclei, we are facing a many-body problem of $N+Z N$ electromagnetically interacting particles. The exact many-body hamiltonian for a 
system is:

$$
\begin{aligned}
\widehat{H}= & \widehat{T}_{n}+\widehat{T}_{e}+\widehat{V}_{e-e}+\widehat{V}_{n-e}+\widehat{V}_{n-n} \\
= & -\frac{\hbar^{2}}{2} \sum_{i} \frac{\nabla_{\vec{R}_{i}}^{2}}{M_{i}}-\frac{\hbar^{2}}{2} \sum_{i} \frac{\nabla_{\vec{r}_{i}}^{2}}{m_{e}} \\
& -\frac{1}{4 \pi \varepsilon_{0}} \sum_{i, j} \frac{e^{2} Z_{i}}{\left|\vec{R}_{i}-\vec{r}_{i}\right|}+\frac{1}{8 \pi \varepsilon_{0}} \sum_{i \neq j} \frac{e^{2}}{\left|\vec{r}_{i}-\vec{r}_{j}\right|}+\frac{1}{8 \pi \varepsilon_{0}} \sum_{i \neq j} \frac{e^{2} Z_{i} Z_{j}}{\left|\vec{R}_{i}-\vec{R}_{j}\right|}
\end{aligned}
$$

The mass of the nucleus at the position $\vec{R}_{i}$ is $M_{i}$, and the mass of the electron at the position $\vec{r}_{i}$ is $m_{e}$. The first two terms are the kinetic energy operators for the nuclei and electrons, respectively; and the last three terms describe the Coulomb interaction between electrons and nuclei, electrons and other electrons, and nuclei and other nuclei. The exact solution of this many-body problem is extremely difficult. Instead, by making some reasonable assumptions it is possible to find acceptable approximate eigenstates.

The first assumption is the Born-Oppenheimer approximation. The nuclei are treated as frozen at their positions $\vec{R}_{i}$ and electrons will be in instantaneous equilibrium with them, because the nuclei are much heavier and slower that the electrons. Therefore in the original many-body problem, the nuclei will treat as an external poential field with fast moving electrons in it. As an consequence, the first term of equation 4.1, the kinetic energy of nuclei will disappear; and the last term, the Coulomb interaction between nuclei and nuclei, will be reduced to a constant. The equation 4.1 can be rewritten as only have three parts (equation 4.2 ), the kinetic energy of electrons $(\widehat{T})$, electron-electron interaction potential energy $(\widehat{V})$, and the 
potential energy of the electrons in the external $\left(\widehat{V}_{\text {ext }}\right)$ potential of the nuclei. For the first two terms of equation 4.2, there is no the effect of nuclei and they are belong to the many-electron system. Therefore the two terms are universal parts and independent of the particular kind of many-electron system. However, the last term of equation 4.2 is system-specific dependent.

$$
\widehat{H}=\underbrace{\widehat{T}+\widehat{V}}_{\text {universal part }}+\underbrace{\widehat{V}_{\text {ext }}}_{\text {system dependent }}
$$

Although Born-Oppenheimer approximation simplifies the original problem, it is still too difficult to solve. Historically, the Hartree-Fock method (HF) works well for atoms and molecules, and frequently used in quantum chemistry. However, when electron correlations effect become prominent, especially in solids, it is less accurate. In order to solve these problems, a new powerful method, know as Density Functional Theory (DFT) was developed. DFT is based on two theorems by Hohenberg and Kohn. The first theorem states that there is a one to one correspondence between the ground state density $\rho(\vec{r})$ of a many electron system and the external potential $V_{\text {ext }}$. Any observable $\widehat{O}$ is a unique functional of the exact ground state electron density (equation 4.3 ):

$$
\langle\Psi|\widehat{O}| \Psi\rangle=O[\rho]
$$

The second theorem states that when $\widehat{O}$ is the hamiltonian $\widehat{H}$, the ground state total 
energy functional $H[\rho] \equiv E_{V_{e x}}[\rho]$ has the form of equation 4.4

$$
\begin{aligned}
E_{V_{\text {ext }}[\rho]} & =\langle\Psi|\widehat{T}+\widehat{V}| \Psi\rangle+\left\langle\Psi\left|\widehat{V}_{\text {ext }}\right| \Psi\right\rangle \\
& =F_{H K}[\rho]+\int \rho(\vec{r}) V_{\text {ext }}(\vec{r}) d \vec{r}
\end{aligned}
$$

The first term in equation $4.4 \mathrm{~b}$ is called Hohenberg-Kohn density functional, which is universal for any many electron system; When the second term $E_{V_{\text {ext }}}[\rho]$ reaches its minimal, it is corresponding to the ground state external potential $V_{\text {ext }}$. Based on this theory, the electron density contains as much information as the wave function does. The electron density can replace the role of wave functions in determining all observable quantities. All observable quantities can be retrieved from the electron density only, so they can be written as the functionals of electron density. The name of the theory comes because all problems now turn to finding the ground state electron density.

Kohn and Sham pushed DFT into a practical tool by a procedure to obtain the ground state density without any approximation. Simply separating the electron exchange and correlation part from Hohenberg-Kohn density functional, the energy functional of equation 4.4 can be rewritten as equation 4.5

$$
E_{V_{e x t}}[\rho]=T_{0}[\rho]+V_{H}[\rho]+V_{x c}[\rho]+V_{e x t}[\rho]
$$

The first term $T_{0}[\rho]$ is the functional for the kinetic energy of a non-interacting electron gas that doesn't include the electron correlation contribution; the second term $V_{H}[\rho]$ is the Hartree contribution that doesn't include the electron exchange contribution; the third term $V_{x c}[\rho]$ is the contribution from electron correlation and 
exchange; and the last term $V_{\text {ext }}[\rho]$ is still the external potential contribution from nuclei. So the corresponding Hamiltonian, the Kohn-Sham Hamiltonian, turns out to be (equation 4.6)

$$
\begin{aligned}
\widehat{H}_{K S} & =\widehat{T}_{0}+\widehat{V}_{H}+\widehat{V}_{x c}+\widehat{V}_{e x t} \\
& =-\frac{\hbar^{2}}{2 m_{e}} \vec{\nabla}_{i}^{2}+\frac{e^{2}}{4 \pi \varepsilon_{0}} \int \frac{\rho(\vec{r} \prime)}{12}|\vec{r}-\vec{r} \prime| d \vec{r}^{2}+V_{x c}+V_{e x t}
\end{aligned}
$$

With such transformation, the problem of many-body related exchange and correlation contributions change to the familiar Schrödinger-like non-interacting single particle question. The exchange-correlation potential has the form of the functional derivative

$$
\widehat{V}_{x c}=\frac{\delta V_{x c}[\rho]}{\delta \rho}
$$

The exact ground state density of $\mathrm{N}$-electron system can be constructed from singeparticle wave functions

$$
\rho(\vec{r})=\sum_{i=1}^{N} \phi_{i}(\vec{r})^{\star} \phi_{i}(\vec{r})
$$

and the single-particle wave functions can be obtained by solving Kohn-Sham equation

$$
\widehat{H}_{K S} \phi_{i}=\varepsilon_{i} \phi_{i}
$$

Since both Hartree operator $V_{H}$ and the exchange-correlation operator $V_{x c}$ depend on the density $\rho(\vec{r})$ and therefore the wave function being searched, we are faced with a self-consistent problem. The self-consistent procedure to obtain the ground state density is illustrate in figure 4.1 


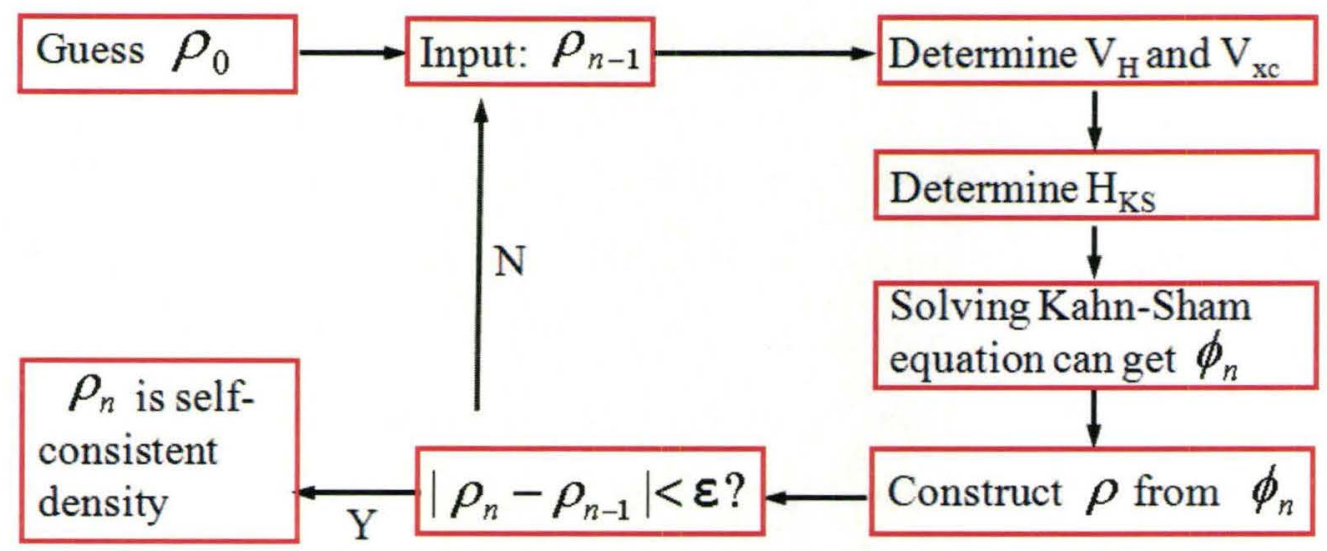

Figure 4.1: Flow chart of self-consistent procedure to solve Kohn-Sham equation

Until this stage, there is no other approximations have been made except the Born-Oppenheimer approximation. Everything looks perfect except the exact form of exchange-correlation functional. However, we have to appeal to the approximations again to find a suitable expression for exchange-correlation functional.

One widely used approximation is the Local Density Approximation (LDA) which assumes the exchange-correlation functional has the following form:

$$
E_{x c}^{L D A}=\int \rho(\vec{r}) \varepsilon_{x c}(\rho(\vec{r})) d \vec{r}
$$

where the function $\varepsilon_{x c}(\rho(\vec{r}))$ is the homogeneous electron gas and can be obtained numerically. The idea of the LDA is that the total exchange correlation energy can be summed over the exchange correlation energies of a homogeneous electron gas in every infinitesimally small volumes with a constant density. Although the LDA is only a reasonable guess and no law of nature guarantees that it is right, it works well for systems with a slowly varying electron density and also very accurate in 
many other cases since it reproduces many fundamental properties of solids.

Another improved approximation from the LDA is the Generalized Gradient Approximation (GGA), which assumes that the exchange correlation energy of every infinitesimal volume is not only dependent on the local density of that volume, but also on the density of the adjacent volumes. Strictly speaking, such a LDA/GGA-calculation is not a first principle calculation any longer but it still parameter free. Now the description of the DFT is complete with some approximations (Born-Oppenheimer approximation and LDA/GGA approximation). The many body problem is converted to many one-electron problems which are easier to be solved effectively with simpler equations.

To solve such Schrödinger-like equations, one has to determine a suitable form of solutions-wave functions, and how many basic sets (wave vectors) you want to use to construct the wave functions. Usually used pseudo-potential form for wave functions describe appropriately outer electrons but not inner electrons. In order to describe both outer and inner electron wave functions, WIEN2k program use Augmented Plane Wave (APW) or Linearized Augmented Plane Wave (LAPW) plus local orbital (lo) method, which we will explain as follows.

For a periodic lattice, according to the Bloch's theorem, any eigenfunction can be written as

$$
\psi_{\vec{k}}^{n}(\vec{r})=\sum_{\vec{K}} c_{\vec{K}}^{n, \vec{k}} f(\vec{K}, \vec{k})
$$

where $c_{\vec{K}}^{n, \vec{k}}$ are coefficients and $f(\vec{K}, \vec{k})$ is a periodic function which usually is the plane wave function. The $\vec{k}$ indicates any vector in the first Brillouin zone, and the $\vec{K}$ 


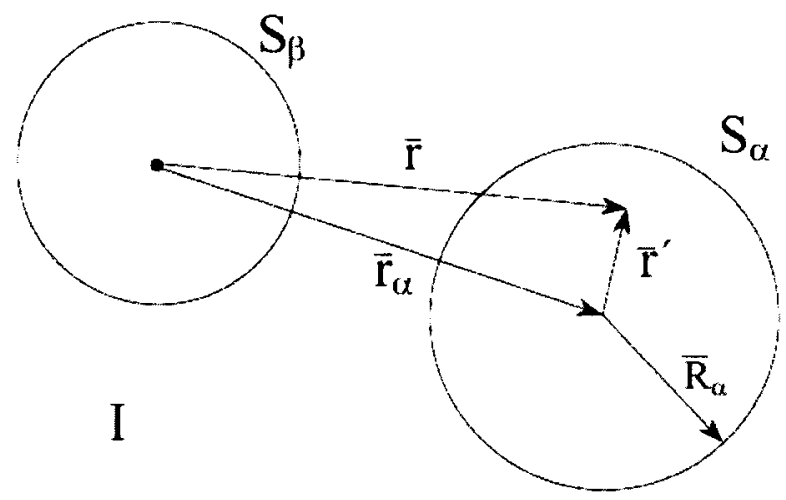

Figure 4.2: Division of a unit cell in muffin tin regions and the interstitial region for a case with two atoms

indicates any vector in reciprocal space. The APW is one method to expand $\psi_{\vec{k}}^{n}(\vec{r})$. The idea is that the space is separated into two regions: in the muffin tin sphere $\left(S_{\alpha}\right)$ where electrons behave as they are in a free atom and can use atomic-like functions to describe them; and the interstitial region (I) (figure 4.2) where electrons can be described by plane wave functions. A typical wave function of single electron in a free atom has the form of $\phi=u_{n, l}(\vec{r}) Y_{l, m}(\theta, \varphi)$, where $u_{n, l}(\vec{r})$ are the radial parts and $Y_{l, m}(\theta, \varphi)$ are spherical harmonics. Therefore the APW used in the expansion of $\psi_{\vec{k}}^{n}(\vec{r})$ has the follow form:

$$
\phi_{\vec{K}}^{\vec{k}}(\vec{r}, E)= \begin{cases}\frac{1}{\sqrt{V}} e^{i(\vec{k}+\vec{K})} \cdot \vec{r} & \vec{r} \in I \\ \sum_{l m} A_{l m}^{\alpha, \vec{k}+\vec{K}} u_{l}^{\alpha}(r \prime, E) Y_{m}^{l}(\widehat{r} \prime) & \vec{r} \in S_{\alpha}\end{cases}
$$

where $A_{l m}^{\alpha, \vec{k}+\vec{K}}$ is an undetermined parameter as is $E$, and $(\vec{k}+\vec{K})$ is any vector in reciprocal space. The position inside the spheres is given with respect to the center of each sphere by $\vec{r} \prime=\vec{r}-\vec{r}_{\alpha}$. The length of $\vec{r} \prime$ is $r \prime$, and the angles $\theta \prime$ and $\phi \prime$ 
representing the direction of $\vec{r} /$ in spherical coordinates, are indicated as $\hat{r}$. As one can see, the APW basis set is $\vec{k}$ dependent. Since the single electron wave function is the sum of $\psi_{\vec{k}}^{n}(\vec{r})$ over all possible $\vec{k}$, the more $\vec{k}$ are chosen, the more accurate the solution can be. However there is a price one has to pay is in term of total calculation time.

To make the calculation more feasible, the Linearized Augmented Plane Wave (LAPW) is introduced, which replace the radial part $u_{l}^{\alpha}$ by expanding it to two terms. So we have the LAPW method form:

$$
\phi_{\vec{K}}^{\vec{k}}(\vec{r})= \begin{cases}\frac{1}{\sqrt{V}} e^{i(\vec{k}+\vec{K})} \cdot \vec{r} & \vec{r} \in I \\ \sum_{l m}\left[A_{l m}^{\alpha, \vec{k}+\vec{K}^{\prime}} u_{l}^{\alpha}\left(r l, E_{1, l}^{\alpha}\right)+B_{l m}^{\alpha, \vec{k}+\vec{K}_{i}} \dot{u}_{l}^{\alpha}\left(r l, E_{1, l}^{\alpha}\right)\right] Y_{m}^{l}\left(\hat{r}^{\prime}\right) & \vec{r} \in S_{\alpha}\end{cases}
$$

where $A_{l m}^{\alpha, \vec{k}+\vec{K}}$ and $B_{l m}^{\alpha, \vec{k}+\vec{K}}$ are undetermined parameters. Since the wave functions have different forms inside and outside muffin tin sphere, the continuity of the wave functions requires that the value and the first order derivative of the two forms are equal at the muffin tin boundary. Now the core state electron wave functions have been well separated from outside the plane wave functions. However, the electron wave functions close to the Fermi level are not strictly pure core states, especially for low-lying valence states, and hence can not be well described by the equation 4.13. A method, called local orbital (lo), is developed by inserting additional information into the radial part of equation 4.13 which is currently best 
approach to treat those semi-core states. The local orbital is defined as:

$$
\phi_{\alpha, L O}^{l m}(\vec{r})= \begin{cases}0 & \vec{r} \in I \\ {\left[A_{l m}^{\alpha, L O} u_{l}^{\alpha}\left(r \prime, E_{1, l}^{\alpha}\right)+B_{l m}^{\alpha, L O} \dot{u}_{l}^{\alpha}\left(r l, E_{1, l}^{\alpha}\right)+\right.} & \\ \left.\left.C_{l m}^{\alpha, L O} u_{l}^{\alpha}\left(r \prime, E_{2, l}^{\alpha}\right)\right] Y_{m}^{l}(\widehat{r} \prime)\right) & \vec{r} \in S_{\alpha}\end{cases}
$$

where $A_{l m}^{\alpha, L O}, B_{l m}^{\alpha, L O}$, and $C_{l m}^{\alpha, L O}$ are undetermined parameters. Of course, the local orbital wave function has to be zero outside muffin tin sphere $\left(S_{\alpha}\right)$. The local orbital is also not $\vec{k}$ dependent. All undetermined parameters in equation $4.12,4.13,4.14$ can be retrieved from the normalization of wave functions and muffin tin sphere boundary conditions.

In summary, the Born-Oppenheimer approximation simply reduces the manybody problem to many-electron problem by treating nuclei's effect as an external field; the DFT change the wave function searches to electron density searches; the Kohn-Sham equation further transforms the many-electron problem to singleelectron problem without any approximation by treating electron exchange and correlation to another external field; LDA or GGA give a good starting performed guess for the electron exchange and the correlation terms; LAPW plus lo methods provide a suitable formal solution for free electron states, core states and semi-core states; the classical treatment for eigenfunction problem, solving secular equations, leads to the solution of Schrödinger equation; finally, the self-consistent scheme helps to find the right electron density. With the right electron density, the quantities of interest can be calculated. 
Under the frame of density functional theory (DFT) with local density approximation (LDA) or improved version of the generalized gradient approximation (GGA), using the full-potential (linearized) augmented plane wave (LAPW) plus local orbitals (lo) methods, the WIEN2k program can carry out the electronic structure calculations. Care should be taken with WIEN2k since it uses different schemes to treat occupied and unoccupied states. The relative energy between two occupied states or two unoccupied states is reasonable if the calculation have been done carefully, but the relative energy between the occupied state and the unoccupied state are known not to be reliable. Band gap energy values from the Wien2k (as in all LDA methods) are underestimated. In spite of these shortcomings, the WIEN2k program is still an extremely powerful tool to explore the material's properties in the scope of electronic structure.

\subsection{Multiplet methods}

Multiplet effects are original atomic effects and are important in X-ray and electron spectroscopies except when $1 s$ core hole presents in the initial of final state. In this chapter, the Fermi Golden rule is introduced first in order to explain the basic aspects about the transition between initial and final states; and then we describes the atomic multiplet with term symbols to determine the possible final states and relative energies between them. These are the most elementary part to be understood. Finally we extend multiplet effects from isolated atom to solid state compounds, with the crystal field model applied as a perturbation to mimic the environment surrounding the atom. 


\subsubsection{Basis aspects}

The probability of transition $W$ between two energy level-initial state $\phi_{i}$ and final state $\phi_{f}$ is determined by the Fermi golden rule (equation 4.15)

$$
W_{f i}=\frac{2 \pi}{\hbar}\left|\left\langle\phi_{f}\left|T_{1}\right| \phi_{i}\right\rangle\right|^{2} \delta\left(E_{f}-E_{i}-\hbar \omega\right)
$$

The delta function represents the energy conservation where a transition takes place only when the energy of the final state is equal to the sum of the energy of the initial state and photon energy. The matrix element gives the transition rate. $T_{1}$ is the transition operator containing the exponential $e^{i k \cdot r}$. Under Taylor expansition, $T_{1}$ can be approximated as $1+i k r+($ higher order terms). In the case of the $K$ edges from carbon $(\mathrm{Z}=6)$ to $\mathrm{zinc}(\mathrm{Z}=30)$, the value of $k \cdot r$ is about 0.04 . Hence the transition probability of electric quadrupole is $2 \times 10^{-3}$ smaller than the dipole transition can be neglected. Then the Fermi golden rule with dipole approximation can be rewritten as equation 4.16

$$
W_{f i} \propto \sum_{q}\left|\left\langle\phi_{f}|q \cdot r| \phi_{i}\right\rangle\right|^{2} \delta\left(E_{f}-E_{i}-\hbar \omega\right)
$$

The Fermi golden rule is a very general expression using the initial state and final state wave functions. The problem is that these wave functions are not exactly known. In practical calculations, approximations are again introduced. An widely used approximation is the single electron approximation, which treats the initial state wave function as a core wave function and the final state wave function as a free electron wave function, simply assuming that all other electrons are not 
involved. It works well only when the interaction between a core hole in the initial state and an electron in the final state is relatively weak. That's the case for $K$ edges where, due to the strongly electronic screening effects, the hole in the initial state can hardly to "feel" the electron in the final state. Therefore the single electron approximation works well for all $K$ edge spectra and excellent agreement for the metal and oxygen $K$ edges are observed. We can use WIEN2k program (see section 4.1) to simulate the $\mathrm{O}-\mathrm{K}$ edge in our experiments.

For other edges, especially the metal $L_{2,3}$ edges, the agreement with WIEN2k is poor. The reason is not because the calculated density of state (DOS) is wrong, but because the DOS changes during the excitation process and the calculated DOS does not account for the change. The reason for the DOS changes in the transition process is due to the strong overlap of the core wave function with the valence wave function. For example, the $2 p$-hole and the $3 d$-hole have radial wave functions that overlap significantly. This wave function overlap is only an atomic effect and can be large. This effect, so-called multiplet effect, is well known in atomic physics and plays a crucial role in the calculation of atomic spectra. The atomic multiplet effects are of the same order in solids as in atoms. A successful method to treat those transitions is based on a ligand-field multiplet model which starts from an atomic model and treat solid state effects as a perturbation. 


\subsubsection{Atomic multiplets}

The Schrödinger equation (equation 4.17) for a free atom contains the kinetic energy of the electrons $\left(p^{2} / 2 m\right)$, the electrostatic interaction of the electrons with the nucleus $\left(Z e^{2} / r\right)$, the electron-electron repulsion $\left(e^{2} / r\right)$ and the spin-orbit coupling of each electron $(l \cdot s)$.

$$
H=\sum_{N} \frac{p_{i}^{2}}{2 m}+\sum_{N} \frac{-Z e^{2}}{r_{i}}+\sum_{p a i r s} \frac{e^{2}}{r_{i j}}+\sum_{N} \zeta\left(r_{i}\right) l_{i} \cdot s_{i}
$$

The first two terms define the average energy $\left(H_{a v}\right)$ for a given atomic configuration, and the last two terms define the relative energy of the different term within a configuration. In addition, the third term-electron-electron repulsion can be separated into spherical average part and non-spherical part. The spherical average part is then added to $H_{a v}$ and the rest electron-electron Hamilton $H_{e e}^{\prime}$ plus $H_{l s}$ determine the relative energies of different terms within the atomic configuration. The final Hamiltonian is as equation 4.18 .

$$
\begin{aligned}
H & =H_{\text {kinetic }}+H_{e n}+H_{e e}+H_{l s} \\
& =\underbrace{H_{k \text { inetic }}+H_{e n}+H_{\text {spherical }}}_{\text {average energy }}+\underbrace{H_{e e}^{\prime}+H_{l s}}_{\text {relative energy }}
\end{aligned}
$$

A so-called term symbol ${ }^{2 S+1} X_{J}$ is used to describe the terms of a configuration, where $X$ equal to $S, P, D$ and $F$ corresponding to $L$ equal to $0,1,2$ and 3 respectively. The $\mathrm{S}$ is the total spin moment and $S=s_{1}+s_{2}+\cdots$; the $\mathrm{L}$ is the total orbital moment and $L=l_{1}+l_{2}+\cdots$; and $\mathrm{J}$ is total moment with $|L-S| \leq J \leq L+S$. With such a 
description, a single $s$ electron is given as ${ }^{2} S_{1 / 2}$ and a single $p$ electron as ${ }^{2} P_{1 / 2}$ or ${ }^{2} P_{3 / 2}$. As to a two-electron configuration, for example $3 d^{1} 4 d^{1}$, due to the nature of the vector, we have $\left|s_{1}-s_{2}\right| \leq S \leq s_{1}+s_{2}$ and $\left|l_{1}-l_{2}\right| \leq L \leq l_{1}+l_{2}$. Since each $d$ electron has $s=1 / 2$ and $l=2$, for $3 d^{1} 4 d^{1}$ configuration, the $\mathrm{S}$ can be 0 or 1 and $\mathrm{L}$ can be $0,1,2,3$ and 4 . The resulting $10 \mathrm{LS}$ term symbols are ${ }^{1} S,{ }^{1} P,{ }^{1} D,{ }^{1} F,{ }^{1} G$, ${ }^{3} S,{ }^{3} P,{ }^{3} D,{ }^{3} F,{ }^{3} G$. Considering spin-orbit coupling, a total of 18 term symbols are found (table 4.1):

Table 4.1: 18 term symbols for $3 d^{1} 4 d^{1}$ configuration

\begin{tabular}{c|ccccc}
\hline & $\mathrm{L}=0$ & $\mathrm{~L}=1$ & $\mathrm{~L}=2$ & $\mathrm{~L}=3$ & $\mathrm{~L}=4$ \\
\hline $\mathrm{S}=0$ & ${ }^{1} S_{0}$ & ${ }^{1} P_{1}$ & ${ }^{1} D_{2}$ & ${ }^{1} F_{3}$ & ${ }^{1} G_{4}$ \\
$\mathrm{~S}=1$ & ${ }^{3} S_{1}$ & ${ }^{3} P_{0}$ & ${ }^{3} D_{1}$ & ${ }^{3} F_{2}$ & ${ }^{3} G_{3}$ \\
& & ${ }^{3} P_{1}$ & ${ }^{3} D_{2}$ & ${ }^{3} F_{3}$ & ${ }^{3} G_{4}$ \\
& & ${ }^{3} P_{2}$ & ${ }^{3} D_{3}$ & ${ }^{3} F_{4}$ & ${ }^{3} G_{5}$ \\
\hline
\end{tabular}

For transition metal ions, the configuration of initial state is $3 d^{N}$; while the configuration of final state, for instance, with a $2 s$ or a $3 p$ core hole, is $2 s^{1} 3 d^{N+1}$ or $3 p^{5} 3 d^{N+1}$. In the case of $3 d^{2}$ configuration, there are total 45 combinations, i.e. $10 \times 9 / 2$, due to the Pauli exclusion principle forbidding two electrons to have the same quantum numbers. The general formula to calculate the degeneracy of a $3 d^{N}$ configuration is

$$
C_{10}^{N}=\frac{10 !}{(10-N) ! N !}
$$

In the most interesting case of the $L$-edge structure ( $2 p \rightarrow 3 d$ transition), it is crucial to understand the configuration of the $2 p^{5} 3 d^{N}$ final state. The term symbols of the $2 p^{5} 3 d^{N}$ states can be found by multiplying the configuration of $3 d^{N}$ with a ${ }^{2} P$ 
term symbol. The total degeneracy of $2 p^{5} 3 d^{N}$ is $C_{6}^{1} \cdot C_{10}^{N}$, for example, when $N=5$, $C_{6}^{1} \cdot C_{10}^{5}=1512$ possible states can be found. Then all these 1512 states are divided into 205 term symbols, implying in principle there are 205 final states. However, not all those final states will show up on the $L$-edge structure. Only the ones obey the selection rule can have finite intensity.

\subsubsection{Matrix elements}

The relative energies of the different terms are determined by calculating the matrix elements of these states with the effective electron-electron interaction $H_{e e}^{\prime}$ and spinorbit coupling $H_{l s}$. The general formulation of the matrix elements of the electronelectron interaction is (equation 4.19)

$$
\left\langle{ }^{2 S+1} X_{J}\left|\frac{e^{2}}{r_{12}}\right|^{2 S+1} X_{J}\right\rangle=\sum_{k} f_{k}\left(l_{i} l_{j}\right) F^{k}\left(l_{i} l_{j}\right)+\sum_{k} g_{k}\left(l_{i} l_{j}\right) G^{k}\left(l_{i} l_{j}\right)
$$

where $F^{k}\left(l_{i} l_{j}\right)$ and $G^{k}\left(l_{i} l_{j}\right)$ are the radial parts of the direct Coulomb repulsion and the Coulomb exchange interaction, respectively. The $f_{k}$ and $g_{k}$ are the angular parts. The direct Coulomb terms are always present whether electrons are in the same or in a different shells; the Coulomb exchange interaction terms are non-zero only for electrons in different shells. The expression of the angular parts can be described by the $3 \mathrm{j}$ and $6 \mathrm{j}$ Wigner symbols:

$$
f_{k}\left(l_{i} l_{j}\right)=\left(2 l_{i}+1\right)\left(2 l_{j}+1\right)(-1)^{L}\left(\begin{array}{ccc}
l_{i} & k & l_{i} \\
0 & 0 & 0
\end{array}\right)\left(\begin{array}{ccc}
l_{j} & k & l_{j} \\
0 & 0 & 0
\end{array}\right)\left\{\begin{array}{lll}
l_{i} & l_{j} & L \\
l_{j} & l_{i} & k
\end{array}\right\}
$$




$$
g_{k}\left(l_{i} l_{j}\right)=\left(2 l_{i}+1\right)\left(2 l_{j}+1\right)(-1)^{S}\left(\begin{array}{ccc}
l_{i} & k & l_{i} \\
0 & 0 & 0
\end{array}\right)\left(\begin{array}{ccc}
l_{j} & k & l_{j} \\
0 & 0 & 0
\end{array}\right)\left\{\begin{array}{lll}
l_{i} & l_{j} & L \\
l_{j} & l_{i} & k
\end{array}\right\}
$$

The matrix elements of the spin-orbit coupling $H_{l s}$ are described as equation 4.20

$$
\left\langle\operatorname{lsjm}\left|\sum_{i=1}^{N} \zeta_{i}\left(r_{i}\right) \vec{l}_{i} \cdot \vec{s}_{i}\right| l \text { sjm }\right\rangle=\sum_{i=1}^{N} \zeta_{i} d_{i}
$$

where $\zeta_{i}$ is radial integral and $d_{j}$ is angular integral. There will be no spin-orbit coupling for a closed-shell. For example, if we neglect $3 d$ shell, the $L_{2}-L_{3}$ splitting from ${ }^{2} P_{1 / 2}$ and ${ }^{2} P_{2 / 3}$ will be

$$
\begin{aligned}
\left\langle l \operatorname{sjm}\left|\zeta_{n l}(\vec{l} \cdot \vec{s})\right| l s j m\right\rangle & =\left\langle l s j m\left|\frac{\zeta_{n l}}{2}\left(\vec{j}^{2}-\vec{l}^{2}-\vec{s}^{2}\right)\right| l s j m\right\rangle \\
& =\frac{\zeta_{n l}}{2}[j(j+1)-l(l+1)-s(s+1)] \\
& = \begin{cases}-\zeta_{2 p} & \text { for } j=1 / 2(l=1 \text { and } s=-1 / 2) \\
\zeta_{2 p} / 2 & \text { for } j=3 / 2(l=1 \text { and } s=1 / 2)\end{cases}
\end{aligned}
$$

The energy difference is $3 \zeta_{2 p} / 2$. The value of $\zeta_{2 p}$ is $3.78 \mathrm{eV}$ for $\mathrm{Ti}^{4+}$ from HartreeFock calculation. Therefore the spin-orbital coupling for $\mathrm{Ti}^{4+}$ is $1.5 \times 3.78=5.67$ $\mathrm{eV}$, which is close to the experimental $L_{2}-L_{3}$ splitting.

\subsubsection{Spectra described with atomic multiplets}

To describe a spectrum with atomic multiplets, we start from a closed shell system $\left(2 p^{6} 3 d^{0}\right)$. The transition of a $2 p$ core electron jumping into the empty $3 d$ shell 
can be described as $2 p^{6} 3 d^{0} \rightarrow 2 p^{5} 3 d^{1}$. According to the description of the term symbols, the ground state $2 p^{6} 3 d^{0}$ has ${ }^{1} S_{0}$ symmetry while the final states $2 p^{5} 3 d^{1}$ can be ${ }^{1} P_{1},{ }^{1} D_{2},{ }^{1} F_{3},{ }^{3} P_{012},{ }^{3} D_{123}$ and ${ }^{3} F_{234}$. The energies of the final states are controlled by the $2 p 3 d$ Slater-Condon parameters, the $2 p$ spin-orbit coupling and the $3 d$ spin-orbit coupling. Thus the transition matrix elements to be calculated are:

$$
I_{(X A S, E E L S)} \propto\left\langle 2 p^{6} 3 d_{\left[{ }^{1} S_{0}\right]}^{0}\left|\vec{r}_{\left[{ }^{1} P_{1}\right]}\right| 2 P^{5} 3 d_{\left[{ }^{1} P_{1},{ }^{1} D_{2},{ }^{1} F_{3},{ }^{1} P_{012},{ }^{3} D_{123},{ }^{3} F_{234}\right]}\right\rangle^{2}
$$

where the symmetry of the dipole transition is given as ${ }^{1} P_{1}$. According to the dipole selection rules that $\Delta J= \pm 1$ or 0 with the exception of $J^{\prime}=J=0$, only the final state with $J=1$ can have finite intensities due to the fact that $J=0$ for the initial state. Therefore only the three terms ${ }^{1} P_{1},{ }^{3} P_{1}$ and ${ }^{3} D_{1}$ are left. The problem is reduced to solving the $3 \times 3$ energy matrix of the final states with $J=1$.

The ground state term symbols of all $3 d^{N}$ systems are given in the table 4.2. As one can see, with the dipole selection rules, the number of final states that can be reached is significantly reduced.

Atomic multiplet theory can accurately describe the $3 d$ and $4 d \mathrm{X}$-ray absorption spectra or EELS spectra of the rare earths. In case of the $3 d$ metal ions, atomic multiplet theory are not accurate any more due to the large effects from the neighbors. The neighbors' configuration-interaction effects therefore must be included. Ligand field multiplet theory thus is therefore used to consider the symmetry effects. 
Table 4.2: The $2 p$ photon or electron transition from ground state to all allowed final state under the dipole selection rule: $\Delta J= \pm 1$ or 0 with the exception of $J^{\prime}=J=0$ (de Groot, 2005a)

\begin{tabular}{llrr}
\hline Transition & Ground State & Allowed Transitions & Possible term symbols \\
\hline $2 p^{6} 3 d^{0} \rightarrow 2 p^{5} 3 d^{1}$ & ${ }^{1} S_{0}$ & 3 & 12 \\
$2 p^{6} 3 d^{1} \rightarrow 2 p^{5} 3 d^{2}$ & ${ }^{2} D_{3 / 2}$ & 29 & 45 \\
$2 p^{6} 3 d^{2} \rightarrow 2 p^{5} 3 d^{3}$ & ${ }^{3} F_{2}$ & 68 & 110 \\
$2 p^{6} 3 d^{3} \rightarrow 2 p^{5} 3 d^{4}$ & ${ }^{4} F_{3 / 2}$ & 95 & 180 \\
$2 p^{6} 3 d^{4} \rightarrow 2 p^{5} 3 d^{5}$ & ${ }^{5} D_{0}$ & 32 & 205 \\
$2 p^{6} 3 d^{5} \rightarrow 2 p^{5} 3 d^{6}$ & ${ }^{6} S_{5 / 2}$ & 110 & 180 \\
$2 p^{6} 3 d^{6} \rightarrow 2 p^{5} 3 d^{7}$ & ${ }^{5} D_{2}$ & 68 & 110 \\
$2 p^{6} 3 d^{7} \rightarrow 2 p^{5} 3 d^{8}$ & ${ }^{4} F_{9 / 2}$ & 16 & 45 \\
$2 p^{6} 3 d^{8} \rightarrow 2 p^{5} 3 d^{9}$ & ${ }^{3} F_{4}$ & 4 & 12 \\
$2 p^{6} 3 d^{9} \rightarrow 2 p^{5} 3 d^{1} 0$ & ${ }^{2} D_{5 / 2}$ & 1 & 2 \\
\hline
\end{tabular}

\subsubsection{The crystal field multiplet}

Crystal field theory is a well-known model to explain the electronic properties of transition metals. It treats the transition metal as an isolated atom surrounded by a distribution of charges that mimic the system, molecule, or solid. This is a very simplistic model but it turns out to be extremely successful in explaining a large range of experiments, such as optical spectra, electron paramagnetic resonance (EPR) spectra and magnetic moments. The most important reason for the success of the crystal field model is that the properties explained are strongly determined by symmetry considerations. Therefore the crystal field model could make full use of the results of groups theory, and group theory also could make a close link to atomic multiplet theory. From the group theory point, the only thing that crystal field theory does is translate (or branch), the results obtained in atomic symmetry to cubic symmetry and further to any other lower point groups. The crystal field is regarded as a perturbation to the atomic multiplet results. Using the group theoretical results, 
the spectral shapes of the ground states can be studied for a solid environment. The crystal field multiplet Hamiltonian extends the atomic Hamiltonian with an electrostatic field term, which is the product of electronic charges and a potential describing the surroundings.

The most encountered case is a transition metal ion surrounded by six equidistant neighboring atoms which are positioned on the three Cartesian axes. They form a so-called octahedral structure, which belongs to the $\mathrm{O}_{h}$ point group. To calculate the multiplet spectra in a cubic crystal, all atomic multiplet matrix elements must be branched to cubic symmetry. The symmetry change from spherical symmetry $\left(\mathrm{SO}_{3}\right)$ to octahedral symmetry $\left(\mathrm{O}_{h}\right)$ causes the $\mathrm{S}$ and $\mathrm{P}$ symmetry states to branch to an $\mathrm{A}_{1 g}$ and $\mathrm{T}_{1 u}$ symmetry state, respectively. The $\mathrm{D}$ symmetry state branches to $\mathrm{E}_{g}+\mathrm{T}_{2 g}$ symmetry state (Figure 4.3 ) in octahedral symmetry and the F symmetry state branches to $\mathrm{A}_{2 u}+\mathrm{T}_{1 u}+\mathrm{T}_{2 u}$. The dipole transition operator has $p$-symmetry and therefore branches to $\mathrm{T}_{1 u}$ symmetry in octahedral symmetry. Based on the interest and our materials, we only focused on the octahedral symmetry $\left(\mathrm{O}_{h}\right)$. For the $\mathrm{O}_{h}$ symmetry, there is only one experimental parameter, $10 \mathrm{Dq}$, which accounts for the crystal field splitting energy. Group theory can predict the splitting, but not the energies of the levels which is related to the type of ions.

We will now replace the symbol term notation by the point group notation, adapted to the new symmetry of the system. The new symbols (symmetry) for transition, initial and final states are those of irreducible representations (IRREP) of the point group. The transformation from atomic multiplets to crystal field multiplets are corresponding to the branching from $\mathrm{SO}_{3}$ symmetry to lower symmetry. For the 


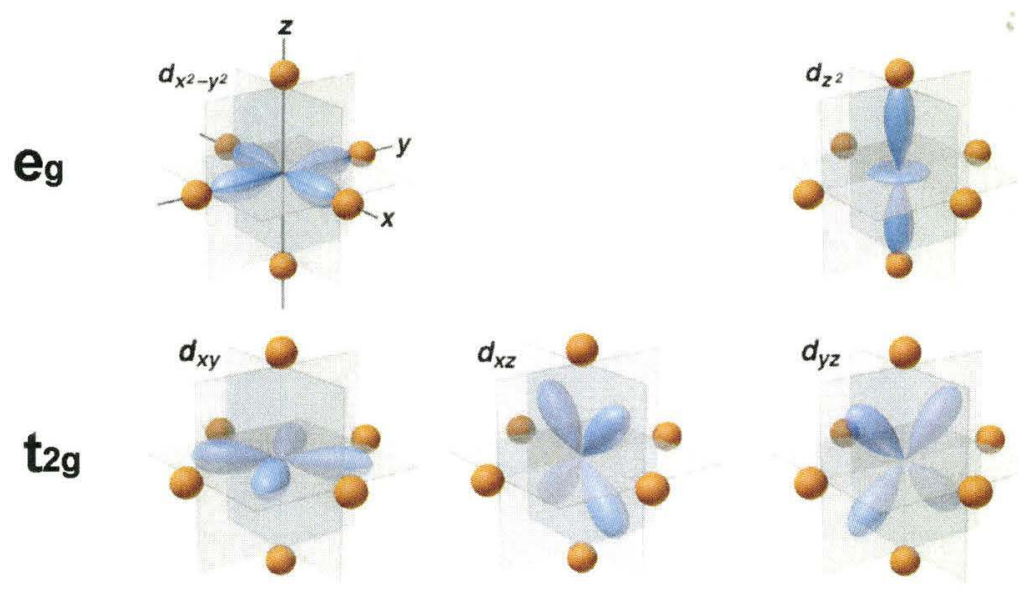

Figure 4.3: $\mathrm{d}$ orbitals splitting in $\mathrm{O}_{h}$ crystal field.

case of $2 p^{6} 3 d^{0} \rightarrow 2 p^{5} 3 d^{1}$ transition in $\mathrm{O}_{h}$ symmetry, the initial state (ground state for $3 d^{0}$ configuration) ${ }^{1} \mathrm{~S}_{0}$ transforms to $\mathrm{A}_{1 g}$, and the dipole operator ${ }^{1} \mathrm{P}_{1}$ transforms as $\mathrm{T}_{1 u}$. As to final state $2 p^{5} 3 d^{1}$, there are 12 terms $\left({ }^{3} \mathrm{~F}_{4},{ }^{3} \mathrm{~F}_{3},{ }^{3} \mathrm{~F}_{2},{ }^{1} \mathrm{~F}_{3},{ }^{3} \mathrm{D}_{3},{ }^{3} \mathrm{D}_{2}\right.$, $\left.{ }^{3} \mathrm{D}_{1},{ }^{1} \mathrm{D}_{2},{ }^{3} \mathrm{P}_{2},{ }^{3} \mathrm{P}_{1},{ }^{3} \mathrm{P}_{0},{ }^{1} \mathrm{P}_{1}\right)$ in atomic multiplets $\left(\mathrm{SO}_{3}\right)$ symmetry before, but when branching to $\mathrm{O}_{h}$ symmetry there are 25 IRREP symbols (table 4.3 ) according to the $J$ value. The lower the symmetry, the lower the degeneracy and therefore more IRREP symbols. The matrix element has the form of $\left\langle\Gamma_{I}\left|\Gamma_{T}\right| \Gamma_{F}\right\rangle$, where $\Gamma_{I}, \Gamma_{T}$,

Table 4.3: 25 IRREP symbols of $2 p^{5} 3 d^{1}$ configuration in $\mathrm{O}_{h}$ symmetry

\begin{tabular}{llc}
\hline$J$ value & IRREP symbol & Times \\
\hline 0 & $\mathrm{~A}_{1 u}$ & 1 \\
1 & $\mathrm{~T}_{1 u}$ & 3 \\
2 & $\mathrm{E}_{u}+\mathrm{T}_{2 u}$ & 4 \\
3 & $\mathrm{~T}_{1 u}+\mathrm{T}_{2 u}+\mathrm{A}_{2 u}$ & 3 \\
4 & $\mathrm{~A}_{1 u}+\mathrm{T}_{1 u}+\mathrm{T}_{2 u}+\mathrm{E}_{u}$ & 1 \\
\hline
\end{tabular}

and $\Gamma_{F}$ are the IRREP of initial state, dipole operator and final state, respectively. The possible transitions from initial state via the dipole operator to the final states 
change from the selection rule to an important group theory result which states that only if the product of the 3 IRREPs, $\Gamma_{I} \otimes \Gamma_{T} \otimes \Gamma_{F}$, contains $A_{1 g}$, the matrix element is non-zero and a transition occur.

Still using the transition $2 p^{6} 3 d^{0} \rightarrow 2 p^{5} 3 d^{1}$ as an example, we can consider the effect of the crystal field. In this case, the product of the 3 IRREPs are $A_{1 g} \otimes T_{1 u} \otimes$ $\Gamma_{F}$. The multiplication table for $\mathrm{O}_{h}$ symmetry (table 4.4) tells us the only possible final state is $\Gamma_{F}=T_{1 u}$. Searching in the table 4.3, there are 7 transitions: 3 from $J=1,3$ from $J=3$ and 1 from $J=4$. Such prediction is in good agreement as comparing to the experimental result (figure 4.4). 4 transitions are more important: 2 peaks for $L_{3}$ edge and 2 peaks for $L_{2}$ due to $e_{g}-t_{2 g}$ splitting.

Table 4.4: Multiplication table of IRREP for $\mathrm{O}_{h}$ symmetry

\begin{tabular}{|c|c|c|c|c|c|c|c|c|c|c|}
\hline $\mathrm{O}_{h}$ & $A_{1 g}$ & $\mathrm{~A}_{1 u}$ & $A_{2 g}$ & $\mathrm{~A}_{2 u}$ & $\mathrm{E}_{u}$ & $\mathrm{E}_{g}$ & $\mathbf{T}_{2 u}$ & $\mathrm{~T}_{2 g}$ & $\mathrm{~T}_{1 u}$ & $\mathrm{~T}_{1 g}$ \\
\hline $\begin{array}{l}\mathrm{A}_{1 g} \\
\mathrm{~A}_{1 u} \\
\mathrm{~A}_{2 g} \\
\mathrm{~A}_{2 u} \\
\mathrm{E}_{u} \\
\mathrm{E}_{g} \\
\mathrm{~T}_{2 u} \\
\mathrm{~T}_{2 g} \\
\mathrm{~T}_{1 u} \\
\mathrm{~T}_{1 g}\end{array}$ & $\mathrm{~A}_{1 g}$ & $\begin{array}{l}\mathrm{A}_{1 u} \\
\mathrm{~A}_{1 g}\end{array}$ & $\begin{array}{l}\mathbf{A}_{2 g} \\
\mathbf{A}_{2 u} \\
\mathbf{A}_{1 g}\end{array}$ & $\begin{array}{l}\mathbf{A}_{2 u} \\
\mathbf{A}_{2 g} \\
\mathbf{A}_{1 u} \\
\mathbf{A}_{1 g}\end{array}$ & $\begin{array}{l}\mathrm{E}_{u} \\
\mathrm{E}_{g} \\
\mathrm{E}_{u} \\
\mathrm{E}_{g} \\
\triangle\end{array}$ & $\begin{array}{c}\mathrm{E}_{g} \\
\mathrm{E}_{u} \\
\mathrm{E}_{g} \\
\mathrm{E}_{u} \\
\nabla \\
\triangle\end{array}$ & $\begin{array}{l}\mathrm{T}_{2 u} \\
\mathrm{~T}_{2 g} \\
\mathrm{~T}_{1 u} \\
\mathrm{~T}_{1 g} \\
\bigcirc \\
\mathbf{0} \\
\square\end{array}$ & $\begin{array}{l}\mathrm{T}_{2 g} \\
\mathrm{~T}_{2 u} \\
\mathrm{~T}_{1 g} \\
\mathrm{~T}_{1 u} \\
\mathbf{0} \\
0 \\
\mathbf{0} \\
\square\end{array}$ & $\begin{array}{l}\mathrm{T}_{1 u} \\
\mathrm{~T}_{1 g} \\
\mathrm{~T}_{2 u} \\
\mathrm{~T}_{2 g} \\
\diamond \\
\vdots \\
\diamond \\
\vdots \\
\square\end{array}$ & $\begin{array}{l}\mathrm{T}_{1 g} \\
\mathrm{~T}_{1 u} \\
\mathrm{~T}_{2 g} \\
\mathrm{~T}_{2 u} \\
\stackrel{0}{ } \\
\dot{8} \\
\diamond \\
\mathbf{\square} \\
\square\end{array}$ \\
\hline \multicolumn{5}{|c|}{$\begin{array}{l}\triangle=\mathrm{A}_{1 g}+\mathrm{A}_{2 g}+\mathrm{E}_{g} \\
\diamond=\mathrm{T}_{2 g}+\mathrm{T}_{1 g} \\
\square=\mathrm{A}_{1 g}+\mathrm{E}_{g}+\mathrm{T}_{2 g}+\mathrm{T}_{1 g} \\
\diamond=\mathrm{A}_{2 g}+\mathrm{E}_{g}+\mathrm{T}_{2 g}+\mathrm{T}_{1 g}\end{array}$} & \multicolumn{6}{|c|}{$\begin{aligned} \nabla & =\mathrm{A}_{1 u}+\mathrm{A}_{2 u}+\mathrm{E}_{u} \\
\mathbf{Q} & =\mathrm{T}_{2 u}+\mathrm{T}_{1 u} \\
& =\mathrm{A}_{1 u}+\mathrm{E}_{u}+\mathrm{T}_{2 u}+\mathrm{T}_{1 u} \\
\mathbf{9} & =\mathrm{A}_{2 u}+\mathrm{E}_{u}+\mathrm{T}_{2 u}+\mathrm{T}_{1 u}\end{aligned}$} \\
\hline
\end{tabular}

The crystal field multiplets only predict possible final states as distinct lines. 


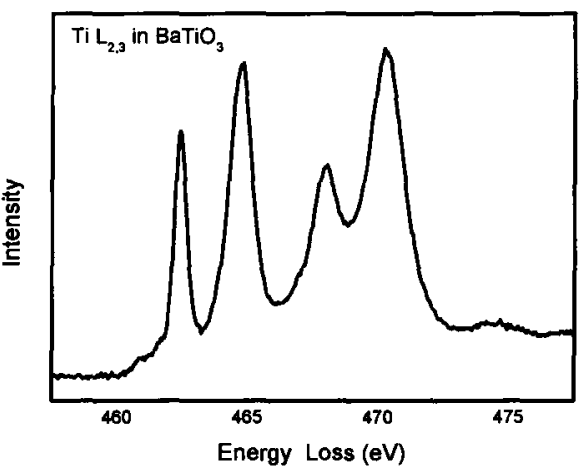

(a)

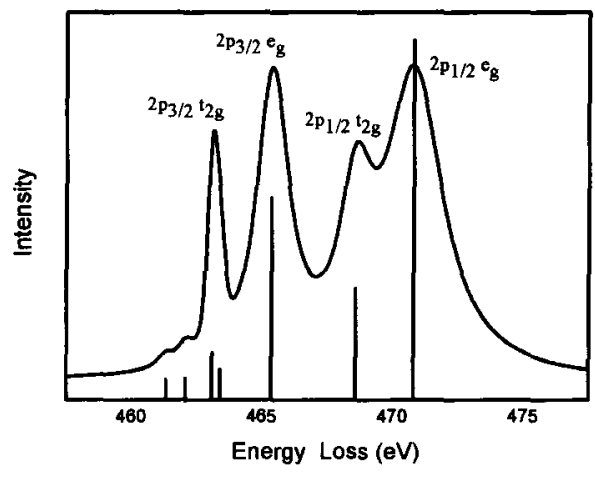

(b)

Figure 4.4: The good prediction of crystal field multiplets. (a) Experimental EELS Ti $L_{2,3}$ edge and (b) crystal field multiplets calculation for Ti $2 p^{6} 3 d^{0} \rightarrow 2 p^{5} 3 d^{1}$ transition in $\mathrm{O}_{h}$ symmetry. The distinct vertical lines are possible final states; and the curve above are the result after broadening

To compare with the experimental data, a few suitable Core-Hole lifetime broadening parameters have to be applied for the corresponding peaks and considered as Lorentzian broadening, as well as the Gaussian broadening for instrument energy resolution. Most of Core-Hole lifetime broadening parameters can be found in the appendix B of the book "Unoccupied electronic states : fundamentals for XANES, EELS, IPS and BIS" (Andrews et al., 1992) from ab inito calculation, and the Gaussian broadening parameter can be obtained from the experiment (FWHM of zero loss peak of EELS). Once again, crystal field multiplets calculation needs some experimental parameters to get the best fit of experimental results. Those experimental parameters are crystal field parameters, Core-Hole lifetime broadening parameters and instrument energy resolution. There is only one crystal field parameter, $10 \mathrm{Dq}$, for $\mathrm{O}_{h}$ symmetry; and 3 crystal field parameter $(\mathrm{Dq}, \mathrm{Ds}$ and $\mathrm{Dt})$ for $\mathrm{D}_{4 h}$ symmetry. The lower symmetry, the more crystal field parameters are needed. Although come with those experimental data, crystal field multiplets calculation 
predicts well for the transition metal ions. More details and complex cases can be found in the review paper "Multiplet effects in X-ray spectroscopy" (de Groot, 2005b). 
PhD Thesis-Yang Shao-McMaster University-Materials Science and Engineering 2009 


\section{Chapter 5}

\section{Thin Films Growth Technique}

There are many techniques to grow thin film samples, such as metal organic chemical vapor deposition (MOCVD), pulsed laser deposition (PLD) and molecular beam epitaxy (MBE). Based on the requirements of having good quality epitaxial films and the instrument available, PLD was selected to grow our $\mathrm{BaTi}_{1-x} \mathrm{Nb}_{x} \mathrm{O}_{3}$ and $\mathrm{Ba}_{1-y} \mathrm{Sr}_{y} \mathrm{Ti}_{0.5} \mathrm{Nb}_{0.5} \mathrm{O}_{3}$ oxide thin films. It has been proved that PLD method can grow high quality single crystal thin films, especially for oxide by carefully choosing a suitable substrate and controlling experimental conditions (Chrisey \& Hubler, 1994). 


\subsection{Preparation of ceramic targets}

In order to apply PLD method to grow thin films, good quality ceramic targets are needed as the source material. For our study, two series of thin films were needed. The ceramic samples of $\mathrm{Ba}_{1-y} \mathrm{Sr}_{y} \mathrm{Ti}_{0.5} \mathrm{Nb}_{0.5} \mathrm{O}_{3}$ and $\mathrm{BaTi}_{1-x} \mathrm{Nb}_{x} \mathrm{O}_{3}$ used as targets for PLD, were prepared by traditional solid solution method. The commercial powders of $\mathrm{SrCO}_{3}, \mathrm{BaCO}_{3}, \mathrm{TiO}_{2}$ and $\mathrm{Nb}_{2} \mathrm{O}_{5}$, were ordered from MTI corporation (http://www.mtixtl.com, Since 1995). According to the desired on the stoichiometry, the appropriate amounts of $\mathrm{SrCO}_{3}(99.9 \%), \mathrm{BaCO}_{3}(99.9 \%), \mathrm{TiO}_{2}(99.9 \%)$ and $\mathrm{Nb}_{2} \mathrm{O}_{5}(99.9+\%$, ultra pure grade) were mixed together first. Afterwards the mixers were fully milled, with a ball mill using 32 pellets of $\mathrm{ZrO}_{2}$ in the solution of isopropanol for at least 24 hours. After milling, the solution is milk-like due to highly dispersed small particles. Then propanal was added to wash out isopropanol, usually for five times. The well dispersed solutions were finally heated to $80^{\circ} \mathrm{C}$ to remove propanal. Here the heating temperature must under the boiling point of the solutions to prevent the uneven composition due to the boiling. The result was an even mixed ivory-white powder with fine particles which can be used for sintering ceramic targets.

The powders were then pressed into pellets and heated at $1250^{\circ} \mathrm{C}$ for 16 hours under a $5 \% \mathrm{H}_{2} / 95 \% \mathrm{Ar}$ flow. The temperature in the furnace was carefully calibrated with respect to different positions and temperature ranges before sintering. The oxygen content can be roughly controlled by mass loss. In total seven targets were obtained with $\mathrm{Nb}$ concentration $\mathrm{x}=0.002,0.02,0.05,0.20,0.50,0.75$ and 1.00 for $\mathrm{BaTi}_{1-x} \mathrm{Nb}_{x} \mathrm{O}_{3}$; and three targets were obtained with $\mathrm{Sr}$ concentration $\mathrm{x}=0.40,0.50$ 
and 0.60 for $\mathrm{Ba}_{1-y} \mathrm{Sr}_{y} \mathrm{Ti}_{0.5} \mathrm{Nb}_{0.5} \mathrm{O}_{3}$. The colors of the ceramic targets were found to change from white before sintering to gray and blue after sintering, indicating the changes of the valence of the ions. The larger the $\mathrm{Nb}$ content is, the darker the sintered ceramic target appears.

\subsection{The pulse laser deposition technique}

The pulsed laser deposition (PLD) method has attracted a lot of attention in the past few years for its ease of use and success in depositing materials of complex stoichiometry. Since the successful deposition of a superconducting $\mathrm{YBa}_{2} \mathrm{Cu}_{3} \mathrm{O}_{7-\delta}$ thin film, many materials normally difficult to deposit by other methods (Dijkkamp et al., 1987; Wu et al., 1987), especially multi-element oxides, have been successfully deposited by PLD.

The main advantage of PLD arises from the laser material removal mechanism. The PLD method relies on a strong photon interaction to create an ejected plasma plume of material from the target. Then the plume condenses on a substrate placed at a short distance from the target. Though the actual physical processes of material removal are quite complex, one can consider the ejection of material to occur due to rapid explosion of the target surface due to superheating by the laser. Unlike thermal evaporation, which produces a vapor composition dependent on the vapor pressures of elements of the target material, the laser-induced expulsion produces a plume with stoichiometry similar to the target. It is generally easier to obtain the desired film stoichiometry for multi-element materials using the PLD. Figure 5.1 
illustrates the schematic drawing of the PLD instrument we used. Although it is

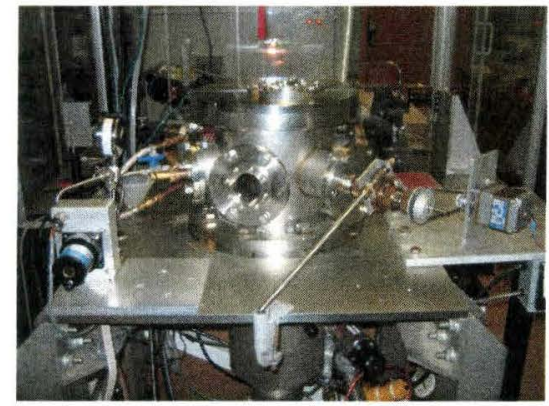

(a) The PLD instrument used in our experiment

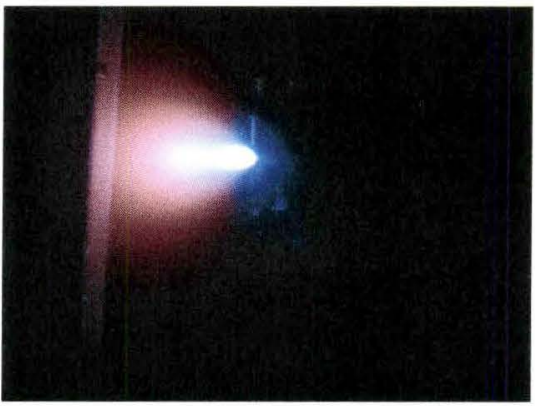

(b) The laser induced plasma plume

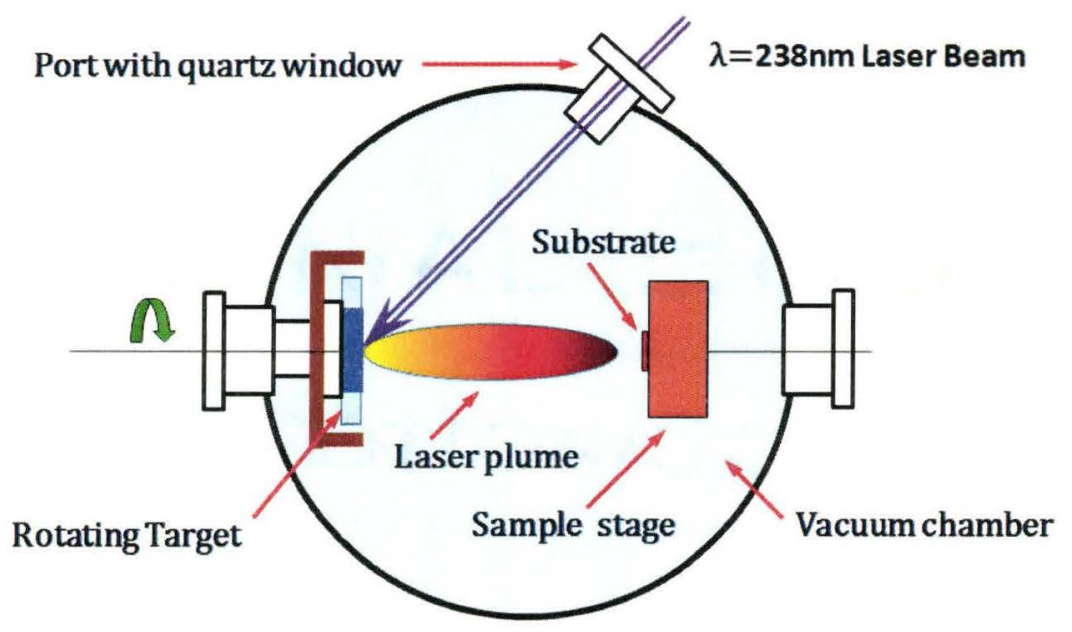

(c) A schematic representation of a PLD instrument

Figure 5.1: The schematic representation of a pulsed laser deposition instrument. (a) The real PLD instrument used for our thin film deposition.(b) The laser induced plasma plume. (c) A schematic representation of a PLD instrument

easier to obtain desired thin films, there are still a lot of experimental parameters one can change to optimize growth condition, such as the energy of each the pulse, the frequency of pulses, the target to substrate distance, the substrate temperature and the gas pressure. All these parameters can be varied from sample to sample. A 
drawback or disadvantage of the PLD method is that it can be only used in lab for depositing small area thin film on a flat substrate. Large area thin film deposition or substrates with varied geometry can not use PLD due to the strongly directed plume.

For our PLD instrument, the GSI Lumonics IPEX-848 excimer laser provides us with a laser wave length of $248 \mathrm{~nm}$, and the optical system provides a $2 \times 3 \mathrm{~mm}^{2}$ laser spot size. Different experiments were carried out to optimize the films quality. We observed (with SEM/XRD and TEM) that the optimum deposition condition for $\mathrm{BaTi}_{1-x} \mathrm{Nb}_{x} \mathrm{O}_{3}$ thin films were at $790^{\circ} \mathrm{C}$ for 20 minutes, with the laser energy of 3 $\mathrm{J} / \mathrm{cm}^{2}$ and repetition rate of $10 \mathrm{~Hz}$. The target to substrate distance is kept at 3.5 $\mathrm{cm}$. With depositions occurring in a vacuum chamber (base pressure of $5 \times 10^{-7}$ torr), film oxygenation relied heavily upon the release of oxygen from the target during the PLD process (Pressure is $2 \times 10^{-6}$ torr). After the deposition the films were cooled in either vacuum or oxygen pressure of 380 torr within the chamber. The typical deposition rates are about $15 \mathrm{~nm} / \mathrm{min}$ for $\mathrm{BaTi}_{1-x} \mathrm{Nb}_{x} \mathrm{O}_{3}$ films and 10 $\mathrm{nm} / \mathrm{min}$ for $\mathrm{Ba}_{1-y} \mathrm{Sr}_{y} \mathrm{Ti}_{0.5} \mathrm{Nb}_{0.5} \mathrm{O}_{3}$ films. 


\section{Chapter 6}

\section{Substrate $\mathrm{MgAl}_{2} \mathrm{O}_{4}$}

The surface of the substrate provides a template for subsequent deposition and growth of thin films, which could impart interface structure, mechanical property and physical properties to films (Ohring, 2002). Due to the ability of the film to attach to substrates, different film growth mechanism will be involved resulting in different film macro-structure. The substrate we used in this work is commercially (001) orientated $\mathrm{MgAl}_{2} \mathrm{O}_{4}$ with a lattice parameters of $8.083 \AA$, melting point of $2130^{\circ} \mathrm{C}$, density of $3.64 \mathrm{~g} / \mathrm{cm}^{3}$ and thermal expensition coefficient of $7.45 \times 10^{-6} / \mathrm{K}$ (from MTI corporation). In this chapter, the surface roughness of $(001)\left(\mathrm{MgAl}_{2} \mathrm{O}_{4}\right.$ as well as the single crystal quality are carefully examined. 


\subsection{The surface roughness}

The surface is a sharp interfacial demarcation between condensed-phase and gasphase (include plasma-phase). The surface topography, such as terraces, facets and roughness, will provide preferred accommodation for atoms sitting at specific positions, and therefore will significantly alter the quality of films as well as generating other orientated grains. Although the specified surface roughness of $\mathrm{MgAl}_{2} \mathrm{O}_{4}$ provided by MTI corporation (http://www.mtixtl.com, Since 1995) is less than $5 \AA$ and samples are packed in 1000 class clean room with 100 class plastic bag, it is still good to determine if there are any facets or terraces created after annealing at high temperature. In addition, real surfaces are usually contaminated with adsorbed gases and assorted compound layers. A cleaning process used to remove all such contaminations list as following:

- First step: 5 mins cleaning by propanol

- Second step: 5 mins cleaning by methanol

- Third step: 5 mins cleaning by acetone

- Forth step: 5 mins cleaning by methanol

Atomic force microscope (AFM) images of $\mathrm{MgAl}_{2} \mathrm{O}_{4}(001)$ surface after the surface cleaning process and annealing at $850^{\circ} \mathrm{C}$, are shown in figure 6.1. Here we notice that the AFM can trace surface topography by detecting the van der Waals force interaction between tip and surface. The surface topography of the area of $1 \mu m \times 1 \mu m$ shown in figure 6.1(a) is very clean (roughness of about $1 \mathrm{~nm}$ ) and 


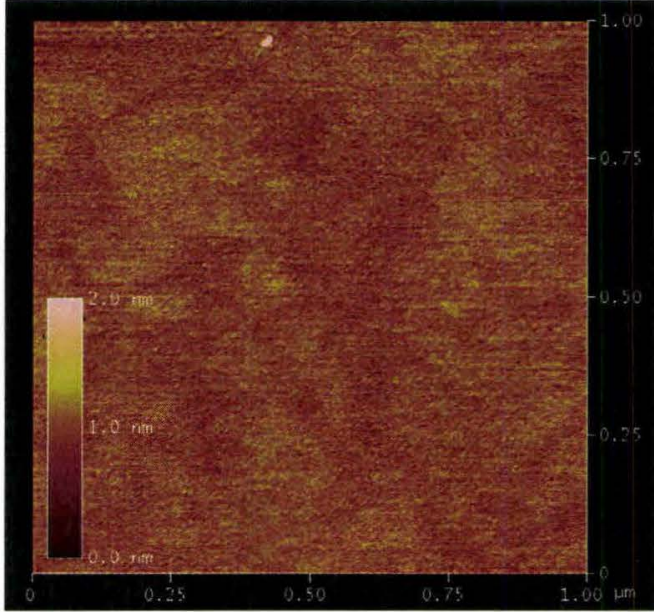

(a)

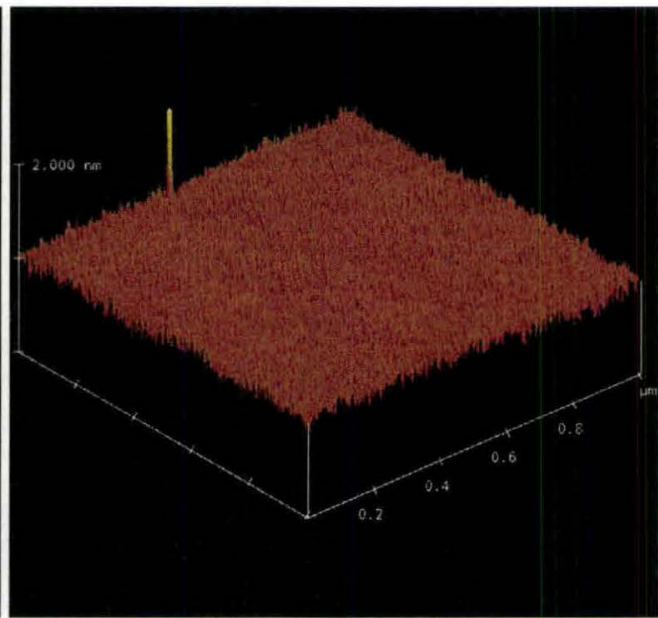

(b)

Figure 6.1: Atomic force microscope image of $(001) \mathrm{MgAl}_{2} \mathrm{O}_{4}$ substrate: (a) surface topography and (b) 3D surface roughness. The respective surface root-mean-square roughness is $0.12 \mathrm{~nm}$. Each division represents a $1 \mu m \times 1 \mu m$ square area

all contaminations are almost removed with the exception of occasional particles within the scanned area, indicating that the cleaning process is effective. We did not detect terraces or facets even after annealing. A smooth 3D surface is shown in figure 6.1(b) except the sharp peak from dust. The color image shows about $1 \mathrm{~nm}$ resolution of our AFM instrument. Therefore within the limit of our AFM instrument, the roughness of $\mathrm{MgAl}_{2} \mathrm{O}_{4}(001)$ surface is about $1 \mathrm{~nm}$ and consistent with the product specification $(<5 \AA)$. 


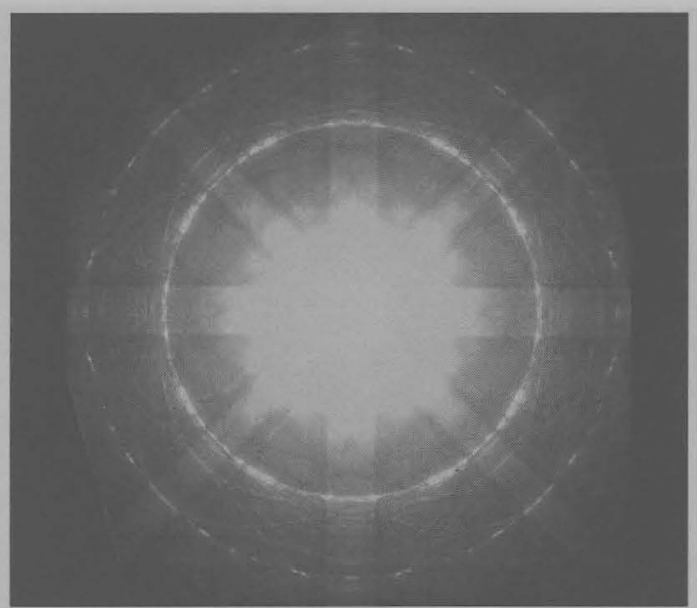

(a) Whole Pattern

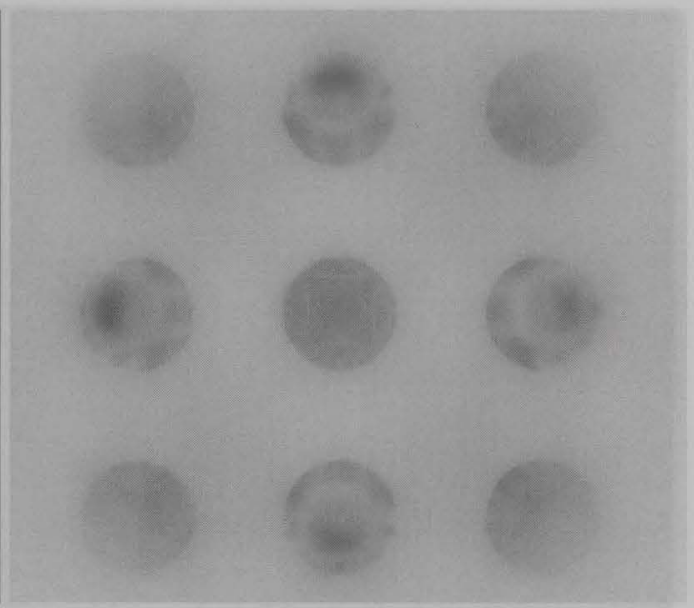

(b) BF pattern

Figure 6.2: $\mathrm{MgAl}_{2} \mathrm{O}_{4}$ substrate CBED patterns of (a) whole pattern (b) bright-field pattern.

\subsection{Crystal structure}

The single crystal quality of $\mathrm{MgAl}_{2} \mathrm{O}_{4}$ was studied by convergent-beam electron diffraction (CBED). Along (001) zone axis, figure 6.2(a) shows a CBED whole pattern with a smaller camera length and figure 6.2(b) shows a bright-field pattern with a larger camera length. Both patterns show a $4 \mathrm{~mm}$ projection symmetry. Together with the XRD results shown later (figure 7.2 on page 94 ), we confirmed that the $\mathrm{MgAl}_{2} \mathrm{O}_{4}$ substrate we used in this work is a good quality single crystal. 


\section{Chapter 7}

\section{$\mathrm{BaTi}_{1-x} \mathrm{Nb}_{x} \mathrm{O}_{3}$ Thin Films on (001) \\ $\mathrm{MgAl}_{2} \mathrm{O}_{4}$ Substrate}

$\mathrm{BaTi}_{1-x} \mathrm{Nb}_{x} \mathrm{O}_{3}$ thin films, spanning the entire range of $x(x=0.002,0.02,0.05,0.20$, $0.50,0.75,1.0$ ), were epitaxially deposited on [001] $\mathrm{MgAl}_{2} \mathrm{O}_{4}$ using the pulsed laser deposition (PLD) technique. In this chapter we will describe the structure characterization first and followed by composition and valence information. Then, transport properties will be presented followed by the electronic structure calculation. Detailed experiment and calculation results will be discussed in the following subsections. 


\subsection{Phase purity}

The structural characterization is obtained through $\theta-2 \theta$ X-ray diffraction (XRD) measurements by using a Bruker D8 advance powder diffractometer with a Ge monochromator at 4 seconds for each scan step of $0.03^{\circ}$. Of considerable interest are the differences observed in the XRD data as both the film's niobium and oxygen content are altered. Figure 7.1 shows the XRD spectra for the $2 \theta$ range near the film's (002) peak for the various film compositions, with the large peak in each figure corresponding to the substrate's (004) reflection. The (004) peak of the substrate is very sharp for all samples and the full width of half maximum (FWHM) is better than 0.1 degree, indicating a good spatial resolution of the XRD instrument. The spectra on the left of the figure correspond to films cooled in vacuum while those on the right were cooled in oxygen. For both cases the peak shifts to lower $2 \theta$ values as the niobium concentration is increased, signifying an increase in the $c$-axis lattice parameter. There exists, however, a significant contraction in the lattice parameter for the $x=1.0$ film cooled in vacuum. Striking is the severe broadening of the $\mathrm{x}$-ray peaks for oxygen cooled films with $x \geq 0.20$. For lower values of $x$ the peak maintains its integrity, but with a slightly smaller lattice parameter than exhibited by the vacuum cooled films.

The large $2 \theta$ range XRD spectra for films cooled in vacuum are shown in figure 7.2. There is no other phase peak from the substrate, confirming that the substrate is single phase. The intensity and FWHM of (002) peak from the films is relatively low and broad with respect to the substrate (002) peak. Only (002) peak prominent 


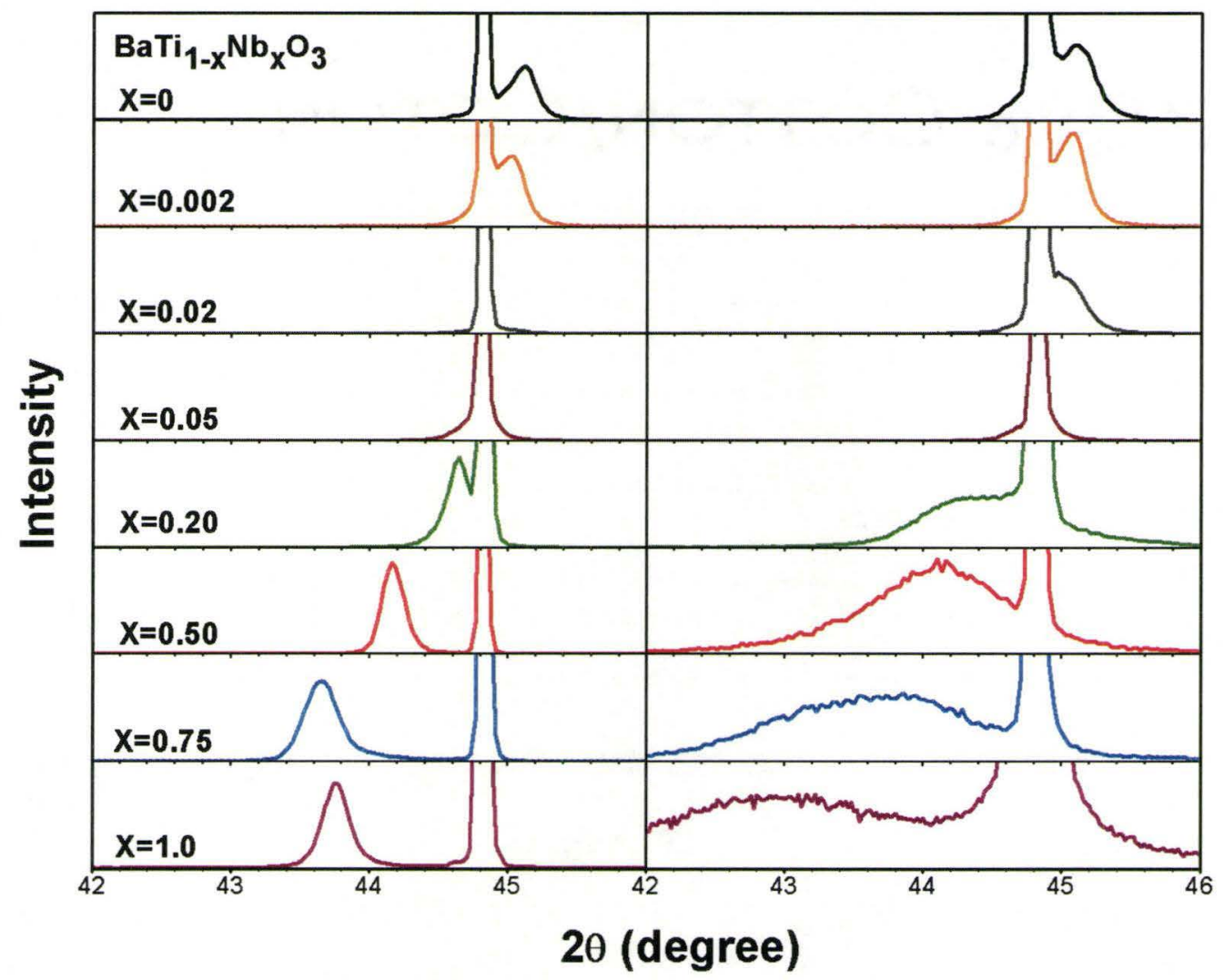

Figure 7.1: $\theta-2 \theta$ XRD data showing the (002) $\mathrm{BaTi}_{1-x} \mathrm{Nb}_{x} \mathrm{O}_{3}$ x-ray peak for film compositions spanning the entire range of $x$. The spectra on left are for films cooled in vacuum while those on the right are for films cooled in 380 Torr of oxygen. Note the shift in the peak towards lower $2 \theta$ values as the niobum concentration is increased as well as the severe degradation in the crystallinity for $x \geq 0.2$ films cooled in oxygen. The large peak at $44.8^{\circ}$ is associated with the substrate's (004) reflection.

for all films indicates that the $\mathrm{BaTi}_{1-x} \mathrm{Nb}_{x} \mathrm{O}_{3}$ films are highly [001] oriented and phase pure, demonstrating for the first time that no solubility limit exists for the substitution of niobium into this compound. There does exist, however, a barely detectable signature emanating from [111] and [011] oriented grains for the $x=0.50$ and $x=1.0$ compositions. The tiny peak from (111) and (011) reflections also mentioned by Liu et al. (2005) for $x=0.50$ composition of their Laser MBE grown film 


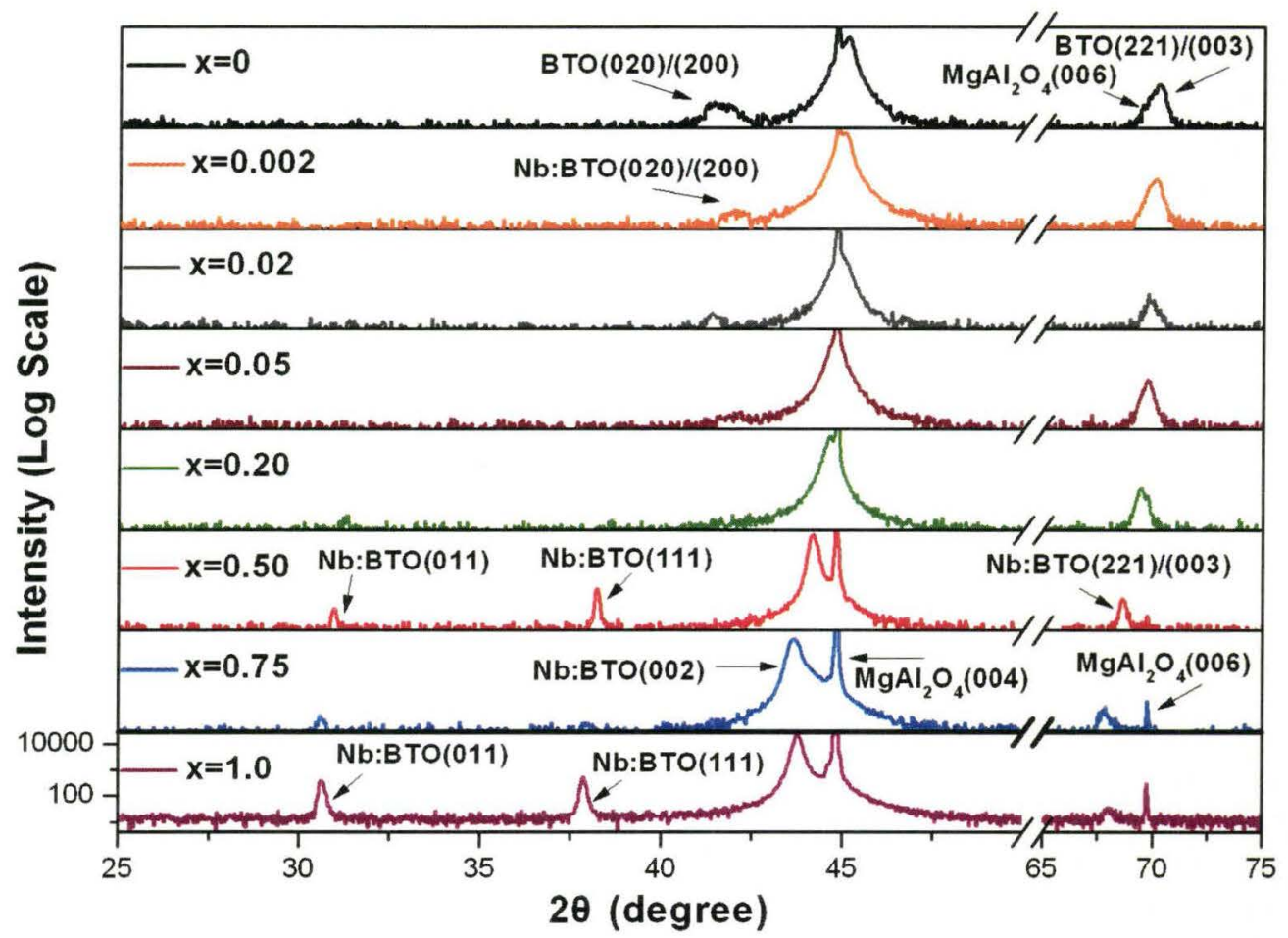

Figure 7.2: The large $2 \theta$ range XRD spectra for $\mathrm{BaTi}_{1-x} \mathrm{Nb}_{x} \mathrm{O}_{3}$ thin films cooling in vacuum. Vertical axis are on a log scale.

on (001) $\mathrm{MgO}$ substrate.

\subsection{Structure and lattice parameter}

The lattice parameter $c$ which is perpendicular to the substrate surface can be simply extracted from XRD data (figure 7.1) and listed in table 7.1. In figure 7.3, we compare the value of the experimental lattice parameter $c$ as a function of $\mathrm{Nb}$ concentration $x$ to the lattice parameter based on the Vegard's law. The value of $c$ sharply 
increases from $x=0$ to $x=0.02$ as the sample is doped with $\mathrm{Nb}$ even at small concentration. After the initial jump, the value of $c$ continues increases up to $x=0.75$ and then decrease for $x=1.0$. The inflexion of $c=4.04 \AA$ around $x=0.02$ indicates the epitaxial effect of the cubic $\mathrm{MgAl}_{2} \mathrm{O}_{4}$ substrate with $a=8.083 \AA$. Thermogravimetric analysis on $\mathrm{BaNbO}_{3-z}$ polycrystalline samples, however, shows that the synthesis of this compound is accompanied by the formation of oxygen vacancies, with $z$ estimated to be $\geq 0.6$ (Gasparov et al., 2001). Such oxygen vacancies could be responsible for the shrinking of lattice parameter $c$ at high $\mathrm{Nb}$ concentration $(x>0.75)$.

Table 7.1: The value of lattice parameter $c$ of $\mathrm{BaTi}_{1-x} \mathrm{Nb}_{x} \mathrm{O}_{3}$ thin films

\begin{tabular}{cc}
\hline $\mathrm{BaTi}_{1-x} \mathrm{Nb}_{x} \mathrm{O}_{3}$ & Lattice parameter $c(\AA)$ \\
\hline $\mathrm{x}=0.002$ & $4.024(2)$ \\
$\mathrm{x}=0.02$ & $4.042(2)$ \\
$\mathrm{x}=0.05$ & $4.043(2)$ \\
$\mathrm{x}=0.20$ & $4.063(2)$ \\
$\mathrm{x}=0.50$ & $4.098(2)$ \\
$\mathrm{x}=0.75$ & $4.142(2)$ \\
$\mathrm{x}=1.0$ & $4.132(2)$ \\
\hline
\end{tabular}

It is also noticed that all $c$ values deviate from the Vegard's law based on the powder samples of $\mathrm{BaTiO}_{3}$ and $\mathrm{BaNbO}_{3}$. As it is, however, the Vegard's law has a hidden requirement that the radii of the ions do not change within the solubility limit. This suggests that our thin films do not meet this requirement as $\mathrm{Ti}$ and $\mathrm{Nb}$ valences are not the same as they are in $\mathrm{BaTiO}_{3}$ and $\mathrm{BaNbO}_{3}$ (see section 7.7 on page 108). It is therefore clear that Vegard's law does not apply in these samples. 


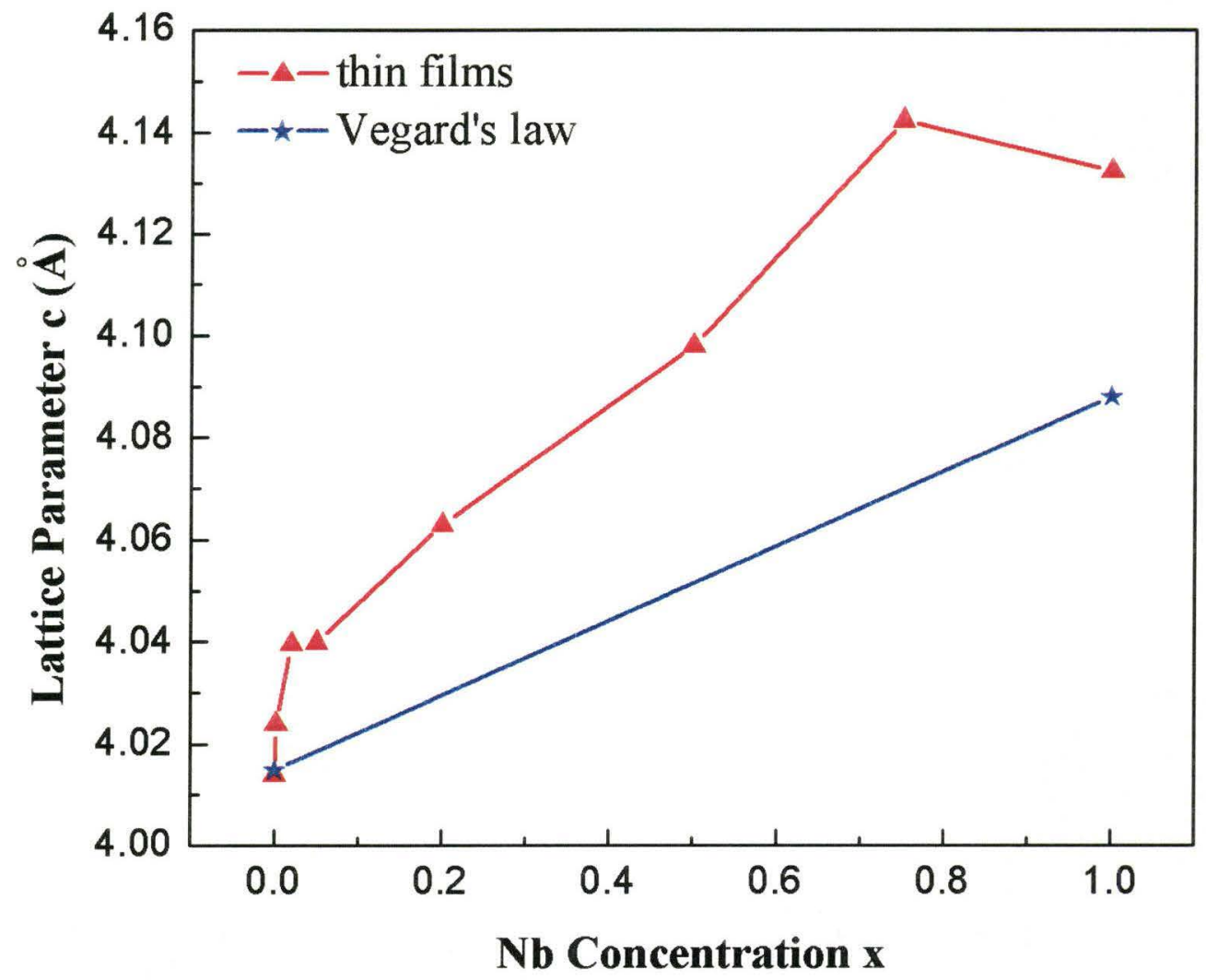

Figure 7.3: Lattice parameter $c$ as a function of $\mathrm{Nb}$ concentration $\mathrm{x}$

In the literature, it was mentioned that $\mathrm{Nb}$ doping can significantly decrease the phase transition temperatures of bulk $\mathrm{BaTiO}_{3}$ from tetragonal to cubic phase, and the low temperature X-ray analysis revealed that the sample remain cubic phase down to $20 \mathrm{~K}$ for $\mathrm{Nb}$ concentration $x \geq 0.10$ (Kolodiazhnyi \& Wimbush, 2006); while a tetragonal to cubic phase transition at $x=0.50$ was found for $\mathrm{BaTi}_{1-x} \mathrm{Nb}_{x} \mathrm{O}_{3}$ $(0.01 \leq x \leq 0.5)$ thin films on MgO substrate, and lattice parameters $a$ and $c$ increased as $\mathrm{Nb}$ concentration increase (figure 2.6 on page 38 ). Our pseudo-cubic structure is also consistent with the bulk samples results of Kolodiazhnyi \& Wimbush (2006) but do not agree with the thin film results of Liu et al. (2005). 


\subsection{Grain orientation}

Grain orientations of thin films were studied with the help of pole figures. The single crystal X-ray diffraction data are collected by a three-circle Bruker D8 diffractometer with a 2D SMART6000 CCD detector. After processing the raw data, the desired pole figures can be generated by the General Area Detector Diffraction System (GADDS). All of the generated pole figures are projected to the $z$ direction which is normal to the surface of the films. More detail work about the grain orientation analysis can be found in Appendix A.

In this section, we only present the most interesting results of the $\{001\},\{011\}$ and $\{111\}$ pole figures for the $x=0.50$ conducting film (see section 7.8 on page 121) on (001) $\mathrm{MgAl}_{2} \mathrm{O}_{4}$ substrate (another example with $x=1.0$ is illustrated in Appendix A. To avoid complexity introduced by other reflections, a narrow integration range was used in the experiments (see table 7.2 on page 97) and was applied to each generated pole figure. Of course, each pole figure will contain orientation information from both the films and the substrate as indicated in table 7.2.

Table 7.2: Summary of the observed reflections for the $2 \theta$ integration windows used in the generation of various pole figures. Because the generation of a pole figure requires a $2 \theta$ integration window, closely spaced periodicities will end up in the same pole figure. Thus, for the pole figures in this chapter, there are peaks corresponding to both film and substrate reflections.

\begin{tabular}{ccl}
\hline Pole figure & Integration range $(2 \theta)$ & Covered reflections \\
\hline$\{001\}$ & $42.3-46.7^{\circ}$ & substrate $(004)+$ film $(002)$ \\
$\{011\}$ & $30.2-32.0^{\circ}$ & substrate $(022)+$ film $(011)$ \\
$\{111\}$ & $36.0-38.0^{\circ}$ & substrate $(311)+$ film $(111)$ \\
\hline
\end{tabular}




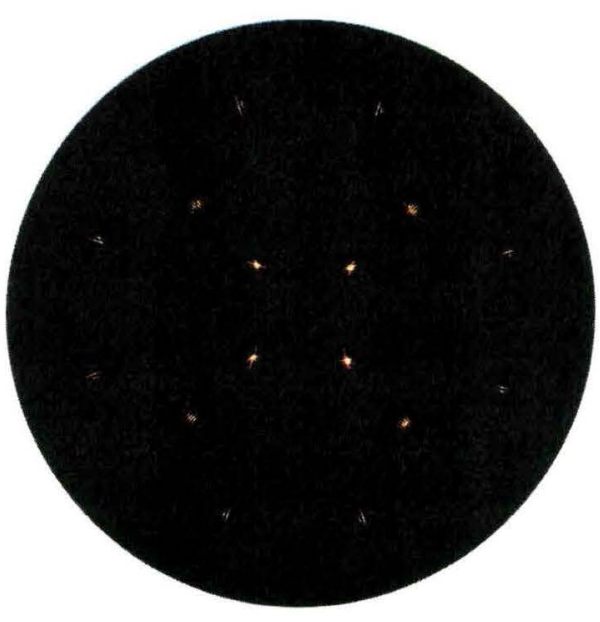

(a) $\mathrm{BaTi}_{1-x} \mathrm{Nb}_{x} \mathrm{O}_{3}$ thin film at $\mathrm{x}=0.50$

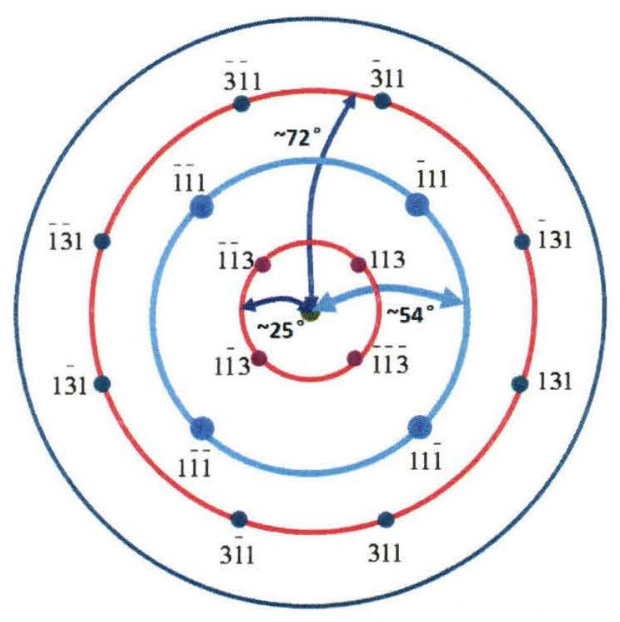

(b) figure of $\{311\}$ and $\{111\}$ poles

Figure 7.4: (a) Pole figures generated from $2 \theta$ range of 36.0-38.0 demonstrates a combination figure of substrate $\{311\}$ poles and film's $\{111\}$ poles for $x=0.50 \mathrm{BaTi}_{1-x} \mathrm{Nb}_{x} \mathrm{O}_{3}$ thin film. (b) An indexed combinational pole figure for one to easily understand the experimental results.

The $\{111\}$ pole figures of $\mathrm{BaTi}_{1-x} \mathrm{Nb}_{x} \mathrm{O}_{3}$ thin films at $x=0.50$ composition is generated in the $2 \theta$ range of $36.0-38.0^{\circ}$ as shown in figure $7.4(\mathrm{a})$. It is a combination of substrate's (311) and film (111) poles as indicated in table 7.2. One can easily identify the highly symmetric 12 sharp (311) poles of the substrate, and 4 strong (111) poles of the films in figure 7.4(b). As we have shown that all our films are highly [001] orientated from $\theta-2 \theta$ XRD data, we still lack of the in-plane grain orientation information. The presence of the 4 (111) poles on the stereoprojection plane without any splitting indicate that the main orientation of the $x=0.50$ $\mathrm{BaTi}_{1-x} \mathrm{Nb}_{x} \mathrm{O}_{3}$ films. There is no in-plane rotation for the main grain orientation. Since the $\mathrm{BaTi}_{1-x} \mathrm{Nb}_{x} \mathrm{O}_{3}$ thin films are deposited and cooled in the same condition, we can expect they are all epitaxial not only in the surface normal direction but also in the in-plane direction, at least for $x=0.50$ composition. 
The $\{001\}$ pole figures of $\mathrm{BaTi}_{1-x} \mathrm{Nb}_{x} \mathrm{O}_{3}$ thin films at $x=0.50$ composition is generated in the $2 \theta$ range of $42.3-46.7^{\circ}$ as shown in figure $7.5(\mathrm{a})$. In this $2 \theta$ range, only the substrate (004) and the films (002) pole are recorded as mentioned in table 7.2. There is only one major spot at the center of the pole figure with twenty-four minor spots symmetrically dispersed around it. Since the substrate is a commercial single crystal, its (004) pole would not contribute to any other spots except the central one in figure 7.5(a). The (002) pole of films' main orientation, however, will also show in the center of stereoprojection plane and merges into the (004) pole of the substrate and therefore can not be distinguished. Besides the major orientation, there are totally nine possible orientations in our films at $x=0.50$ composition. Each of the nine possible different orientations was shown in figure 7.5(b), represented by the same symbol and connected by lines. The first set of orientation is represented by a big solid circle at the center, the four second set of orientations ( $<111>$ orientated grains) are represented by the same solid symbol and connected by the solid line, and the four third set of orientation $(<221>$ orientated grains) are represented by the same open symbol and connected by the dash line. The nine possible orientations could arise from lattice misfit between substrate and films or non-optimized deposition condition. Such highly symmetric $\{001\}$ pole figure suggests there may exist twin structure. The $<221>$ reflection showed on figure 7.2 around $69^{\circ}$ and might overlap with (003) reflections for our pseudo-cubic structure because the $d$-spacing is the same for both (221) and (003). The $<221>$ orientated grain could be due to forming twin structures with the main orientation (see section 7.4 on page 102).

The $\{011\}$ pole figures of $\mathrm{BaTi}_{1-x} \mathrm{Nb}_{x} \mathrm{O}_{3}$ thin films at $x=0.50$ composition is 


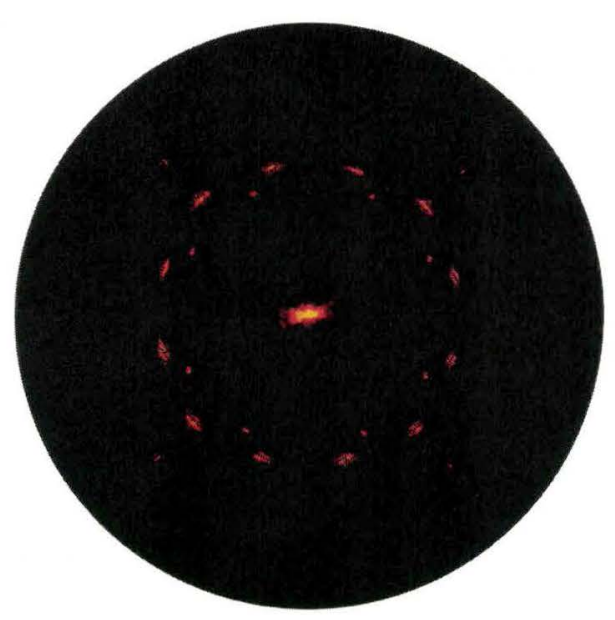

(a) $\mathrm{BaTi}_{1-x} \mathrm{Nb}_{x} \mathrm{O}_{3}$ thin film at $\mathrm{x}=0.50$

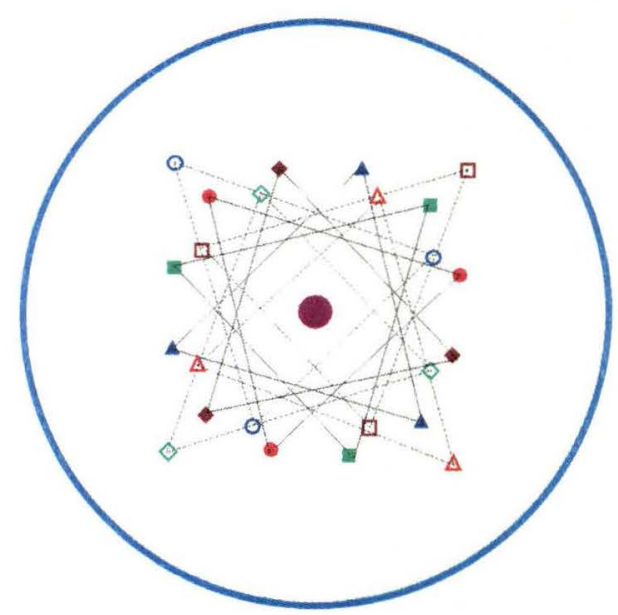

(b) A possible nine-orientation model

Figure 7.5: Films' $\{001\}$ pole figures for $\mathrm{BaTi}_{1-x} \mathrm{Nb}_{x} \mathrm{O}_{3}$ thin film at $x=0.5$, generated from $2 \theta$ range of $42.3-46.7^{\circ}$ (a) $\mathrm{BaTi}_{1-x} \mathrm{Nb}_{x} \mathrm{O}_{3}$ thin film at $x=0.50$ composition, and (b) A possible nine-orientation model presented to explain the $\{001\}$ pole figure. Each orientation is represented by the same symbol and connected by the corresponding lines.

also generated in the $2 \theta$ range of $30.2-32.0^{\circ}$ as shown in figure $7.6(\mathrm{a})$. There are four strong poles sitting on the single crystal (011) pole positions and twenty-four other poles symmetrically around. Such pattern of the pole figure is because of more orientations in the films which have been clarified in in previous $\{001\}$ pole figure (figure 7.5$)$. In the $\{011\}$ pole figures, the (011) poles of films' main orientation are also overlapping with substrate $(011)$ poles. To illustrate the relationship between other poles is more difficult but the basic idea is the same as what we have explained in the $\{001\}$ pole figure (figure $7.5(b)$ ). The difference is that $(011)$ poles are not normal to each other and we have to use Wulff net as an assistance. Since $\{001\}$ pole figure is more easy to understand the grain orientations and there is no more information we can get from $\{011\}$ pole figure. Here we only present the final result in the figure 7.6(b). Of course, again, nine grain orientation are found. The same 


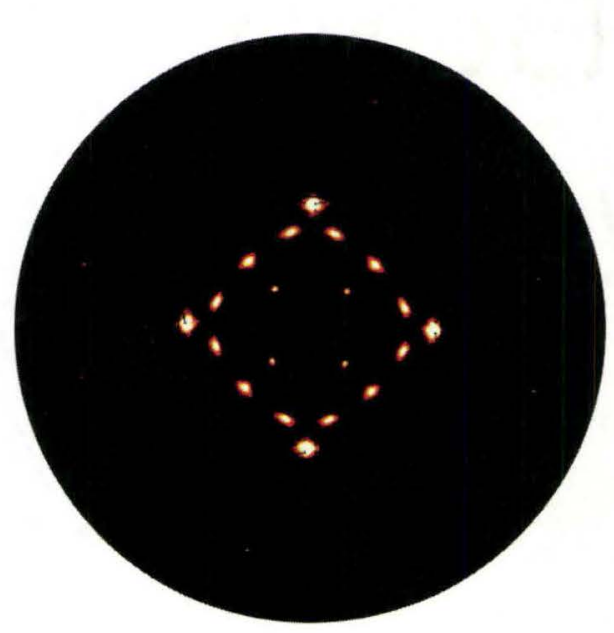

(a) $\mathrm{BaTi}_{1-x} \mathrm{Nb}_{x} \mathrm{O}_{3}$ thin film at $\mathrm{x}=0.50$

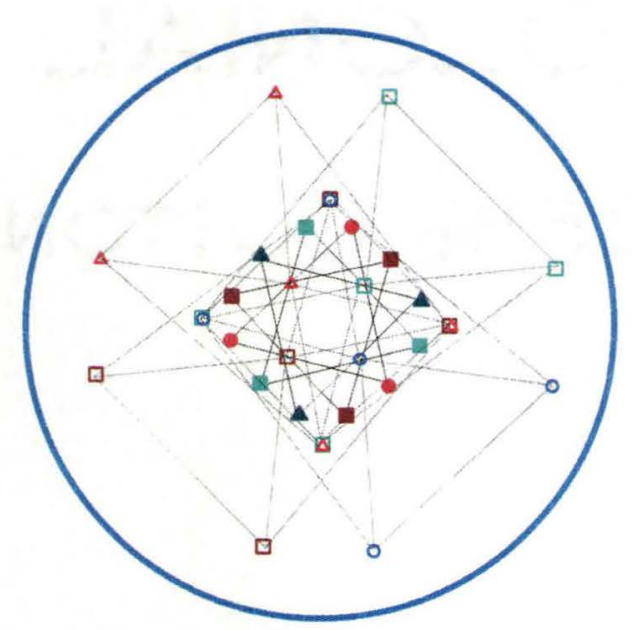

(b) A possible nine-orientation model

Figure 7.6: Films' $\{011\}$ pole figures for $\mathrm{BaTi}_{1-x} \mathrm{Nb}_{x} \mathrm{O}_{3}$ thin film at $x=0.5$ composition, generated from $2 \theta$ range of $30.2-32.0^{\circ}$. Nine possible grain orientations are confirmed. (a) $\mathrm{BaTi}_{1-x} \mathrm{Nb}_{x} \mathrm{O}_{3}$ thin film at $\mathrm{x}=0.50$ composition (b) Nine possible grain orientations are confirmed. Each orientation is represented by the same symbol and connected by the corresponding lines.

orientations are marked with same symbols and connected with lines. Moreover, for convenience, the same grain orientation in the $\{001\}$ (figure $7.5(b))$ and $\{011\}$ (figure 7.6(b)) pole figures is marked by the same type of symbols. It is clearly to see that the third set orientations $(<221>$ orientated grains $)$ as described in figure 7.5(b) by the same open symbol and connected by the dash line, share two $<011>$ directions with the main orientation and form twin structure (see section 7.4 on page 102). 


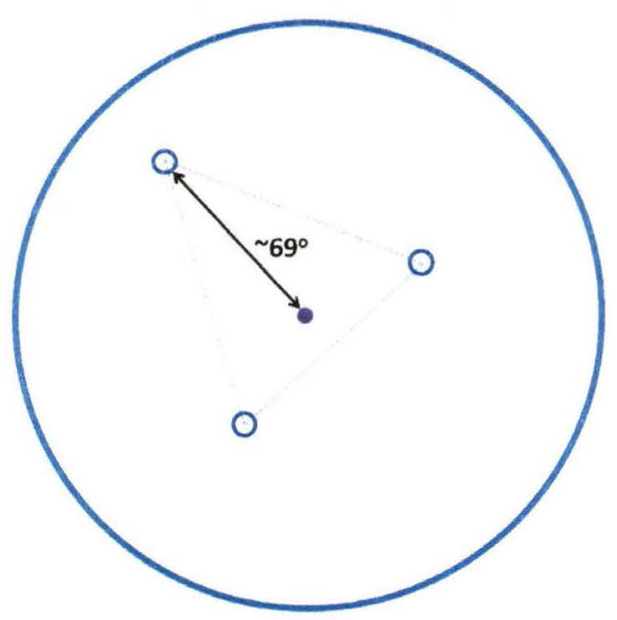

(a) (001) pole figure

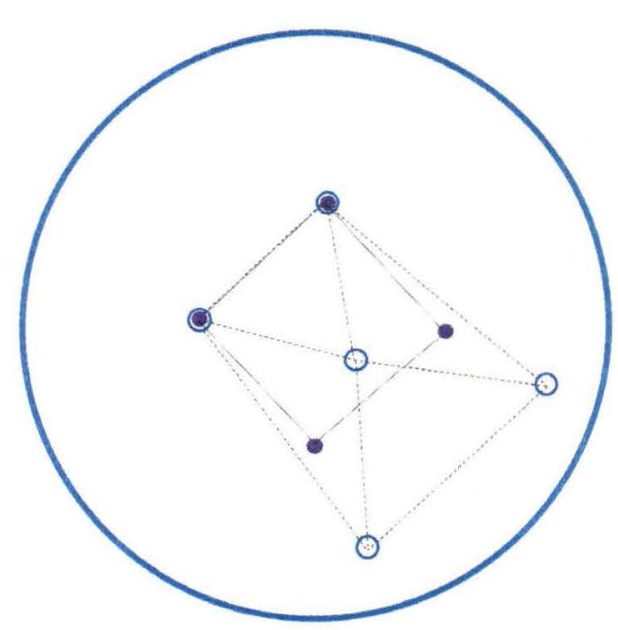

(b) (011) pole figure

Figure 7.7: The orientation relationship between one minor and major grain in (a) (001) pole figure and in (011) pole figure

\subsection{Twin Structure}

From the data we have obtained in the previous section 7.3 grain orientation, possible twin structures are predicted. Here we give more details about the twin structure and provide additional evidence. The same one of third set of grain orientations is outlined in both (001) and (011) pole figures (figure 7.7) with the surface normal in the $<221>$ direction as well as the major grain orientation with (001) surface normal. The relative tilt angle between such two grains is around $69^{\circ}$ measured from figure 7.7(a). In figure 7.7(b), it is easily found that two $<011>$ poles are shared by the two grains. Inasmuch as the two grains shared two non-parallel directions, they have to share one plane. By cross product of the two $<011>$ directions, we know the shared plane is $\{111\}$. Since totally four third set of grain orientations exist in the film, four such twin structures can be expected. 


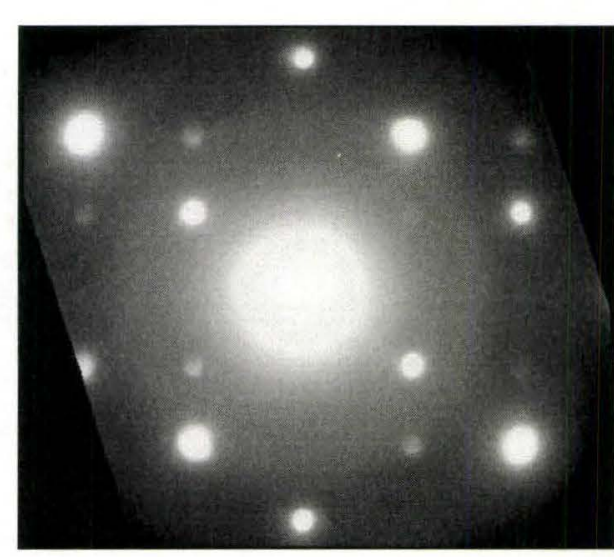

(a)

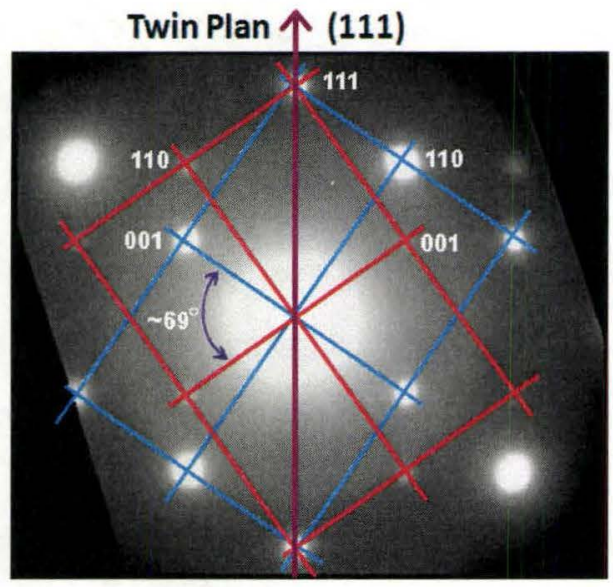

(b)

Figure 7.8: Twin structure found by diffraction pattern along (11̄0) zone axis. (a) the typical twin structure diffraction pattern (b) the labeled diffraction pattern

Another evidence is presented in figure 7.8 from the CBED pattern at [1 $\overline{1} 0]$ zone axis obtained with a small C2 aperture and small spot size for the $x=0.50$ composition thin film. Figure 7.8(a) is a typical twin structure diffraction pattern shown by the presence of a mirror plane regardless of the spots intensities. The indexed pattern of the possible superimposed orientations shown in figure 7.8(b). The shared mirror plane is (111) which is consistent with what we find from (011) pole figure figure 7.7(b); and the angle of $69^{\circ}$ between [001] directions of two supposed lattice is also consistent with the angle obtained from the (001) pole figure (figure 7.7(a)).

In addition, all the four second set of orientations (from minor grains) are orientated in $<111>$ direction and hence share $\{111\}$ plane. If those minor grains are connected to each other, twin structures can be expected by sharing $\{111\}$ plane; 
on the other hand, if those minor grains are separated by major grains and isolated in the films, they can not form twin structure. However, we don't have any microstructure image or diffraction pattern to support the evidence of this, and of course, we cannot eliminate it. Here we only present such possibility. Such minor $\{111\}$ orientated grains were disappeared when the deposition temperature increased from $790-850^{\circ} \mathrm{C}$ (see figure B.1(a) on page 204 ).

\subsection{TEM analysis}

TEM cross section bright field images of films at $x=0.50,0.75$ and 1.0 composition are shown in figure 7.9. The thicknesses of thin films is about $300 \mathrm{~nm}$, and this information will be used later in section 7.8 to determine the resistivity of thin films. The films show good epitaxy on the substrate with some columnar grains. The width of the columnar structures is around $50 \mathrm{~nm}$.

The columnar grain growth of thin films has been a topic of great interest for several decades. This microstructure consisting of a network of low density material surrounding an array of parallel rod-shaped columns of higher density. Columnar structures appear when the lateral mobility of the deposited atoms is limited and the films grow faster in the direction to the surface normal. Their occurrence is therefore ubiquitous. Columnar structures have been found in high melting point materials ( $\mathrm{Cr}, \mathrm{Si}$ and $\mathrm{Ge}$ ) as well as in high bonding energy compound ( $\mathrm{TiC}, \mathrm{TiN}$ and $\mathrm{CaF}_{2}$ ) (Ohring, 2002). The existence of the columnar structures open the possibility of transverse fracture of the films due to the crack propagation along the boundaries 
regions. If such cracks partial occur (not necessarily cross the whole film), the mechanical, electrical and magnetic will be affected. However, the columnar structure can be sometimes reduced by increase the lateral mobility of adatoms on the substrate by increase deposition temperature or use suitable substrate. Although the columnar structures are clearly visible in our bright field images 7.9 , their orientations just slightly tilted from the main orientation, showing extra reflections around the center in the $\{001\}$ pole figure 7.5.

We have checked the composition in the film and in the different columns for $x=0.50,0.75$ and 1.0 by energy dispersive X-ray spectroscopy. It was found there was no composition change between the film and columns, indicating that columns within structure have the same composition as the film. Here we only showed EDS results for $\mathrm{x}=0.50$ film under reducing condition in figure 7.10.

\section{6 $\mathrm{Nb}$ ions distribution by diffraction pattern}

The $\mathrm{Nb}$ ordering and substitution for $\mathrm{Ti}$ atoms has the potential to affect the resistivity by changing the electron transportation network. In the literature, there are no report of ordering reflection found for ceramic $\mathrm{BaTi}_{1-x} \mathrm{Nb}_{x} \mathrm{O}_{3}$ samples at low concentration $(x<0.1)$ based on the diffraction patterns analysis. The possible ordering phases have been illuminated in section 2.3. Three B-site sublattice types for 1:1 ordered perovskites are posted as in figure 2.2: a rock salt structure, a layered structure and a random structure. The preferred structure results from a 

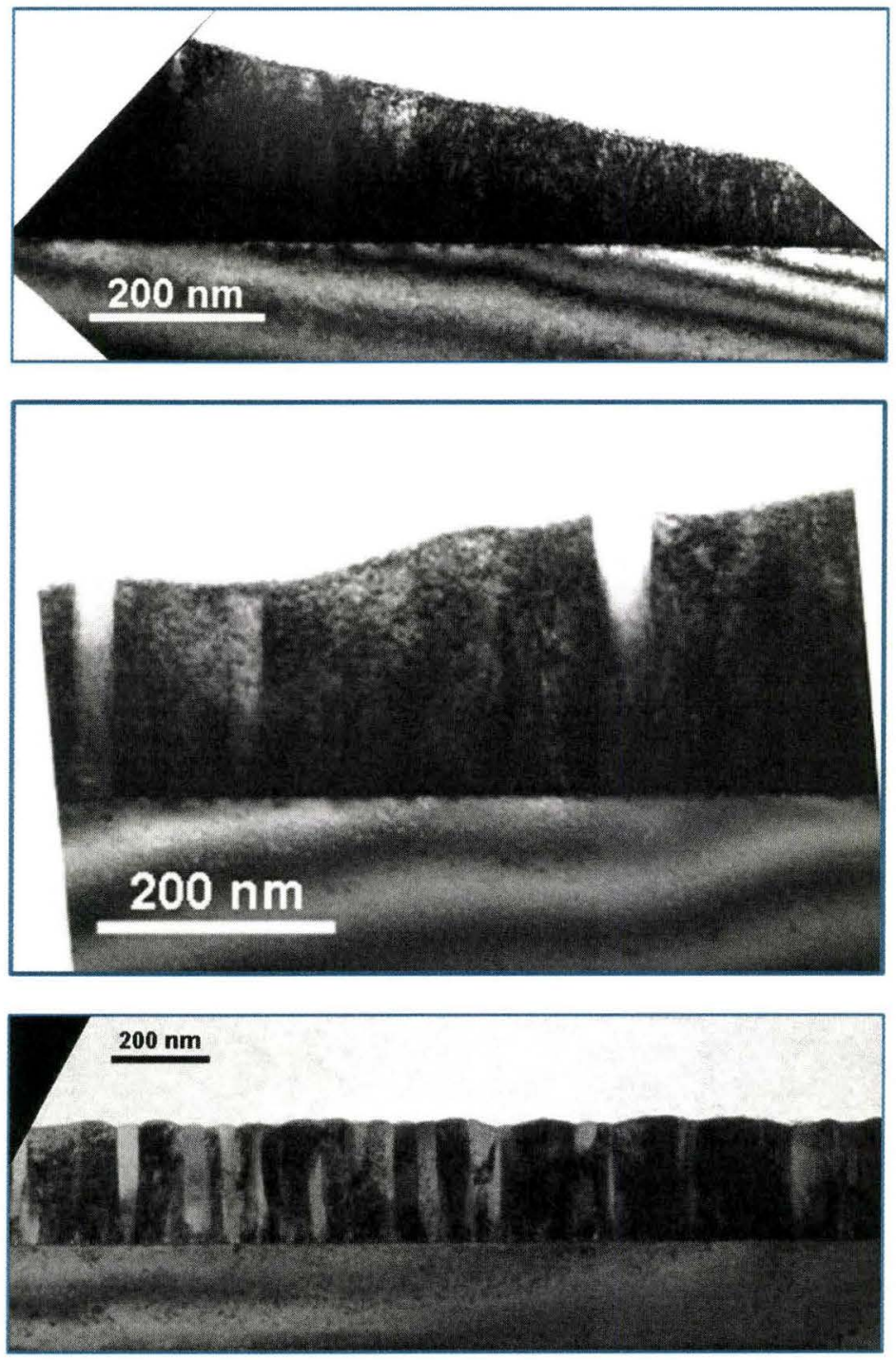

Figure 7.9: TEM bright field image of $\mathrm{BaTi}_{1-x} \mathrm{Nb}_{x} \mathrm{O}_{3}$ thin film at (a) $x=0.50$, (b) $x=0.75$ and (c) $x=1.0$ composition. Of note, (a) is the edge of the foil and the thickness of the film is not changing as shown in the image. It is an effect of the sample preparation.

balance among the B-site cation radii, the Madelung energy and the system entropy. According to the figure 2.3 and the examples shown in the table 2.1, B-site cations with small charge difference and similar radius size favor random arrangement. The 

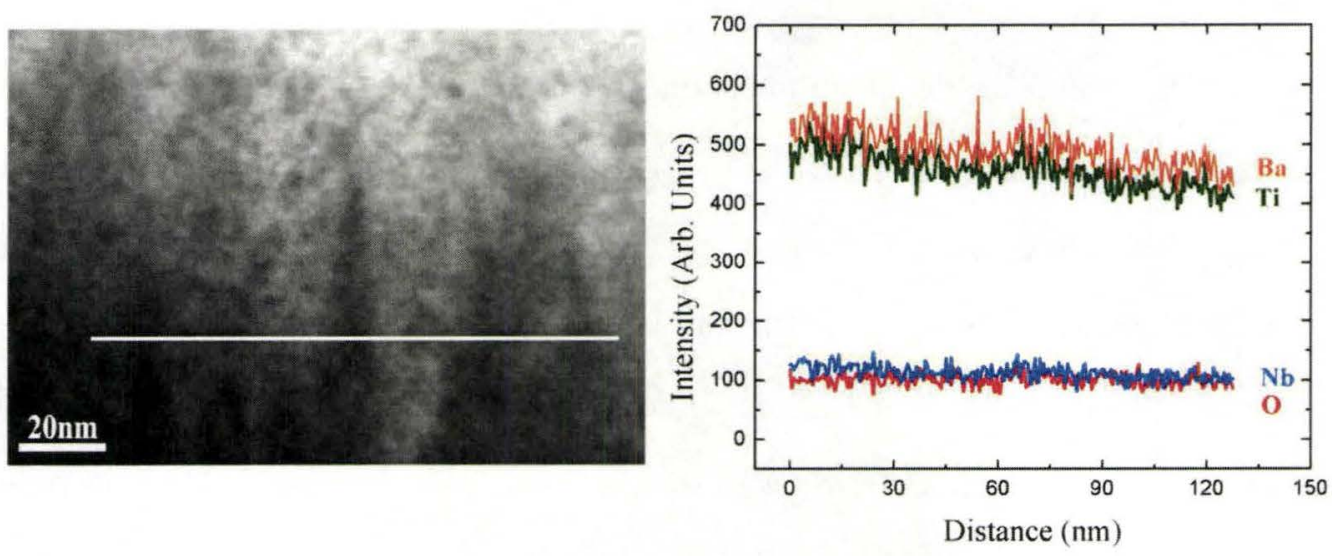

Figure 7.10: Energy Dispersive $\mathrm{X}$-ray spectroscopy line scan analysis of $\mathrm{BaTi}_{1-x} \mathrm{Nb}_{x} \mathrm{O}_{3}$ thin film at $x=0.50$ composition

radius size difference of $\mathrm{Nb}$ and $\mathrm{Ti}$ ions is $3 \mathrm{pm} \mathrm{for} \mathrm{Nb}^{5+}$ and $\mathrm{Ti}^{3+}$, and $7.5 \mathrm{pm}$ for $\mathrm{Nb}^{4+}$ and $\mathrm{Ti}^{4+}$. For the $x=0.5$ sample, based on the valence and ionic size, we would expect a random arrangement of $\mathrm{Nb}$ ions. However, we can provide additional experimental information to support our expectations based on the valence since such conclusion is very important to understand the transport properties.

We used electron diffraction patterns to provide information on ordering. To obtain a good result and insure the diffraction pattern coming from only one grain, convergent-beam electron diffraction (CBED) technique was used with a spot size of $30 \mathrm{~nm}$. A smallest condenser aperture size is applied to prevent the diffraction disc overlapping. In this case, the CBED pattern appears similar to the traditional selected area diffraction (SAED or SAD) patterns but the beam can be focused onto a much smaller area.

If there is ordering in the structure, superlattice reflections will be observed from particular zone axes or the radius of higher-order laue-zone will shrink. For 
our $x=0.5$ and 0.75 composition conducting films, three diffraction patterns from different zone axises ([001], [011], [111]) are shown in figure figure 7.11(a), 7.11(b) and 7.11(c), respectively. The patterns were taken in CBED mode in the Philips CM12 microscope with a large camera length and long exposure time in order to increase the visibility of any chance of superlattice. The diffraction patterns with a small camera length are also taken (figure 7.11(d), 7.11(e) and 7.11(f)) in order to identify the position of high-order laue-zone. Simulated results with an assumed random structure conducted by JEMS program are shown in figure $7.11(\mathrm{~g}), 7.11(\mathrm{~h})$ and 7.11(i).

Our results show that there are no superlattice reflections found in the CBED patterns (figure 7.11(a), 7.11(b) and 7.11(c)) and even the first-order laue-zone (figure 7.11(d), 7.11(e) and 7.11(f)) fits well with our simulated results assuming with B-site cation random distribution model (figure 7.11(g), 7.11(h) and 7.11(i)). All these results suggest that there is no ordered structure in our films and the $\mathrm{Nb}$ atoms randomly replace Ti atoms on B-sites.

\subsection{Valence state analysis by EELS}

\subsubsection{Ti-L 2,3 edge}

In order to assess the titanium oxidation state, Ti- $L_{2,3}$ edge spectra were recorded on a FEI Tecnai 200 FEG microscope equipped with a monochromator and a high- 


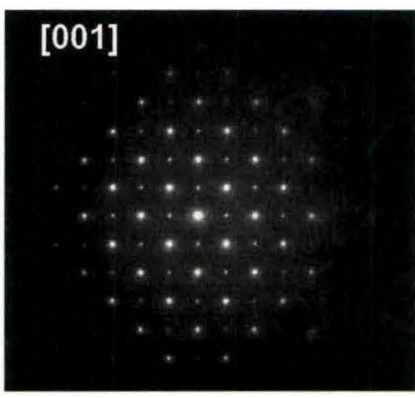

(a)

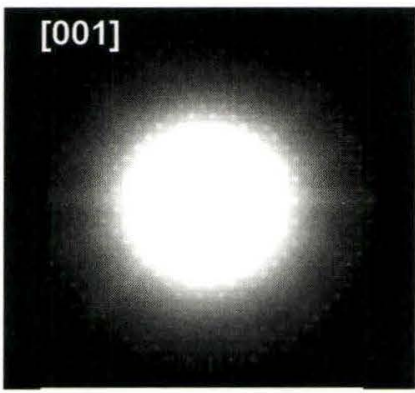

(d)

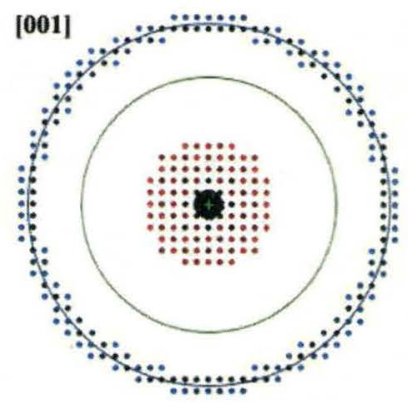

$(\mathrm{g})$

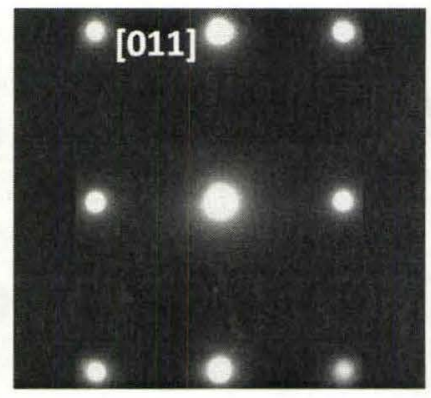

(b)

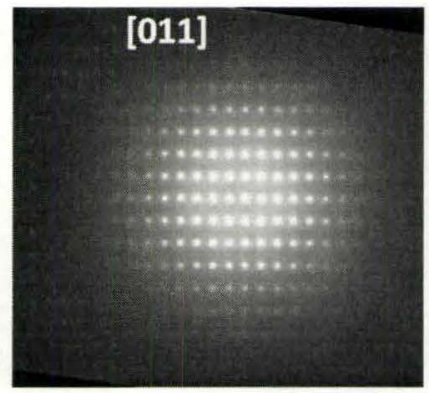

(e)

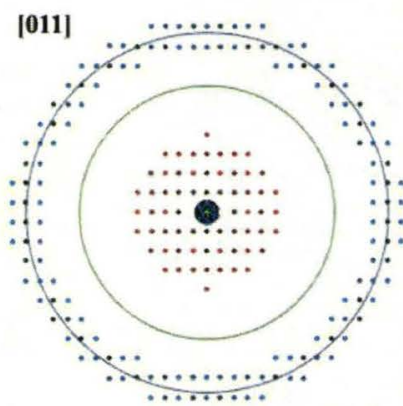

(h)

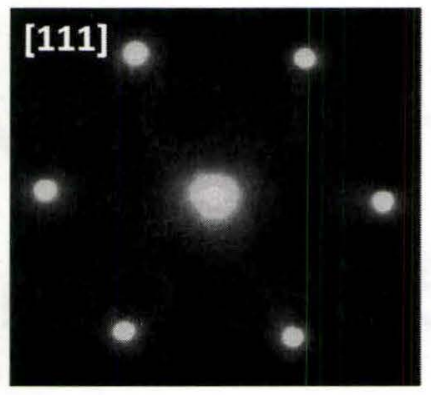

(c)

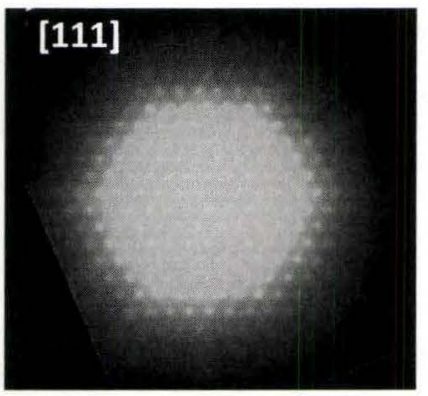

(f)

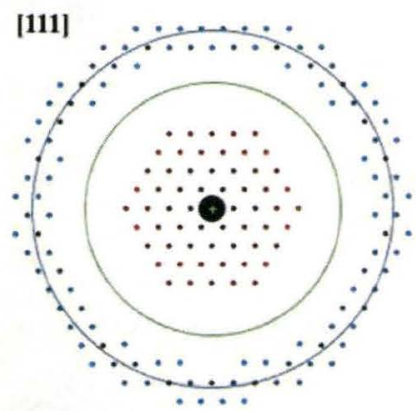

(i)

Figure 7.11: CBED patterns compared to the simulated results with assumed B-site ordered structure for $\mathrm{BaTi}_{1-x} \mathrm{Nb}_{x} \mathrm{O}_{3}$ thin film at $\mathrm{x}=0.5$ and 1.0 composition. CBED pattern taken with a large camera length at (a) 001 zone-axis (b) 011 zone-axis and (c) 111 zone-axis and with a small camera length at (d) 001 zone-axis (e) 011 zone-axis and (f) 111 zone-axis. Simulated diffraction patterns with assumed B-site random structure by JEMS at (g) 001 zone-axis (h) 011 zone-axis and (i) 111 zone-axis

resolution energy-loss spectrometer. The EELS resolution using this system is up to $0.1-0.2 \mathrm{eV}$ measured by the full width at half maximum (FWHM) of the zero loss 


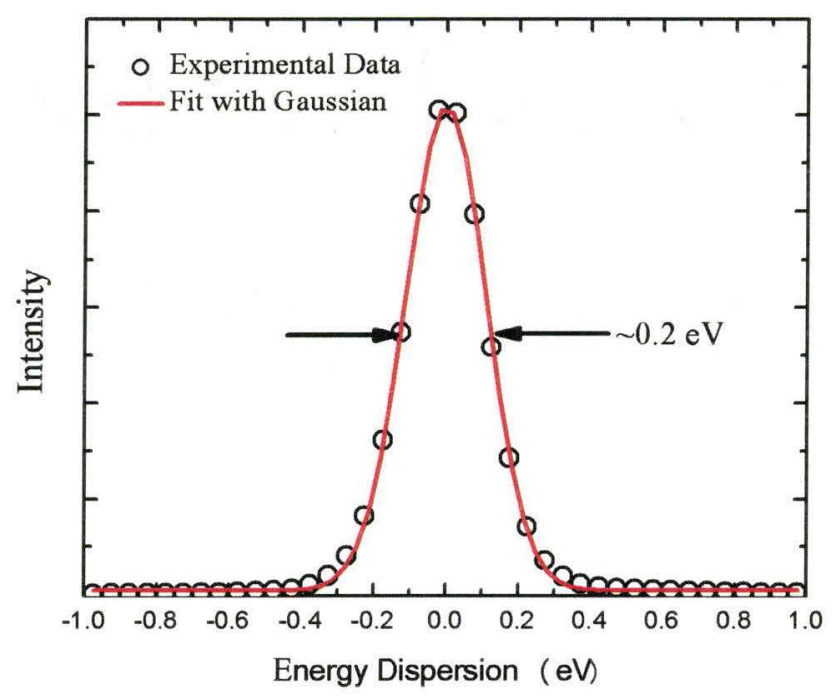

Figure 7.12: Zero loss peak of a high-resolution energy-loss spectrometer system we used to record EELS spectra. The experimental data (open circle spots) are fitted by Gaussian peak (red solid line). The FWHM of the fitted peak is around $0.2 \mathrm{eV}$

peak (figure 7.12). The spatial resolution of our experiment is limited within a few tens of nanometers. We acquired the spectra in diffraction mode with a large collection angle ( $>10$ mard) to eliminate the anisotropic effect, and a energy dispersion of $0.05 \mathrm{eV} / \mathrm{Channel}$. The total acquisition time varies between $50-300 \mathrm{~s}$ by adding a series of spectra acquired with relatively short acquisition time (each between 5-30 s). The background was extrapolated using the power law and then extracted by the standard procedure. We ensure that our sample is thin enough to neglect the multiple inelastic scattering contributions. The Ti- $L_{2,3}$ edge of $\mathrm{BaTi}_{1-x} \mathrm{Nb}_{x} \mathrm{O}_{3}$ thin film at $x=0.50$ and 0.75 composition as well as $\mathrm{BaTiO}_{3}$ are shown in figure 7.13 - The experimental Ti- $L_{2,3}$ edge of $\mathrm{BaTiO}_{3}$ can be used as a reference for $\mathrm{Ti}^{4+}$. The shape of Ti- $L_{2,3}$ edge of $\mathrm{BaTiO}_{3}$ is a characteristic of a d $\mathrm{d}^{0}$ transition metal in octahedral (or slightly distorted octahedral) symmetry. The prominent four peaks 
can be explained in a simple one electron picture: firstly, the $2 p$ spin-orbit coupling gives rise to two separated peaks known as $L_{3}$ and $L_{2}$ edge; then each of the two peaks splits into two peaks again representing two energy levels $\left(t_{2 g}\right.$ and $\left.e_{g}\right)$ of a $3 d$ band, due to the effect of octahedral crystal field. However, the single electron picture fails to explain the fine structures observed near transition metal $L_{2,3}$ edges. A more explicit treatment of electron-electron interactions and ligand field multiplets calculations as usually applied for this edge (de Groot, 2005b). For the $L_{2,3}$ edge of $\mathrm{Ti}^{4+}$ in an octahedral crystal field with a $10 \mathrm{Dq}$ of $1.80 \mathrm{eV}$, in addition to the four prominent peaks there are also some fine peaks are well separated from the main ones. A specific Lorentzian broadening parameters are applied for each peaks based on the values obtained from the compound $\mathrm{FeTiO}_{3}$ (de Groot et al., 1990).

The spectra of $x=0.5$ and $x=0.75$ show significant alterations with respect to $\mathrm{BaTiO}_{3}$. The multiplet calculations, as well as previous experimental observations for mixed valence compounds (Abbate et al., 1991; Schofield et al., 1995), reveal that the changes to the spectra are associated with a $\mathrm{Ti}^{3+}$ oxidation state within an octahedral crystal field. While we cannot completely rule out the existence of the $\mathrm{Ti}^{4+}$ oxidation state as a minor concentration for the $x=0.5$ and $x=0.75$ films, it is apparent from the data that its role in the structure has been drastically reduced.

Besides the thin samples, some polycrystalline ceramic $\mathrm{BaTi}_{1-x} \mathrm{Nb}_{x} \mathrm{O}_{3}$ samples with low $\mathrm{Nb}$ content $(x \leq 0.2)$ are also investigated. These samples were provided by Taras Kolodiazhnyi (National Institute for Materials Science, Japan) and prepared by solid state reaction from high purity $99.99+\% \mathrm{BaCO}_{3}, \mathrm{TiO}_{2}$, and $\mathrm{Nb}_{2} \mathrm{O}_{5}$. Sintering was performed in forming gas $\left(3 \% \mathrm{H}_{2}+97 \% \mathrm{~N}_{2}\right)$ to ensure electronic rather than 


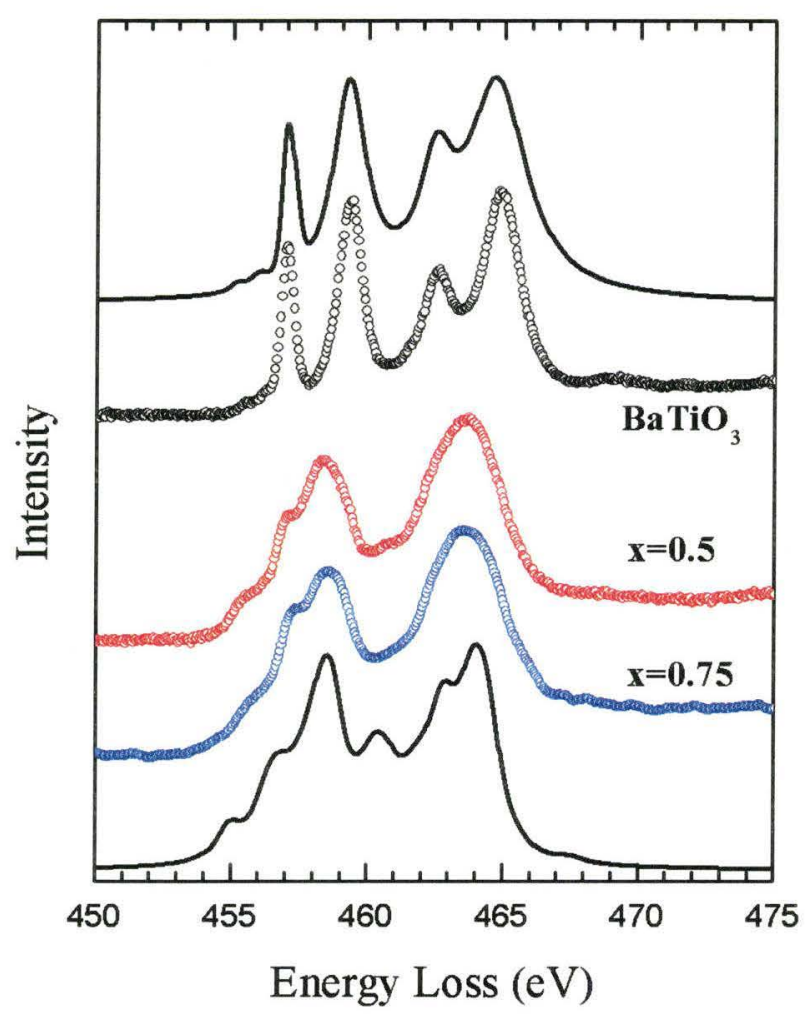

Figure 7.13: Ti- $L_{2,3}$ edge spectra for $\mathrm{BaTi}_{1-x} \mathrm{Nb}_{x} \mathrm{O}_{3}$ thin films at $x=0.50$ and 0.75 composition and for $\mathrm{a} \mathrm{BaTiO}_{3}$ standard. Also shown are the calculated multiplet spectra (solid lines), assuming octahedral symmetry, for the $\mathrm{Ti}^{3+}$ (bottom curve) and $\mathrm{Ti}^{4+}$ (top curve) oxidation states. The results are consistent with a transformation in the oxidation state from $\mathrm{Ti}^{4+}$ to $\mathrm{Ti}^{3+}$ as niobium is substituted for titanium.

cation vacancy compensation of the $\mathrm{Nb}$ dopant, which is very similar to our target preparation (see section 5.1 on page 82 ). The Ti- $L_{2,3}$ edge spectra was recorded on a FEI Titan 80-300 microscope equipped with a monochromator and a highresolution energy-loss spectrometer. The energy spread of the incident beam in a single spectrum taken with a $1 \mathrm{~s}$ exposure time at FWHM was $0.24-0.27 \mathrm{eV}$. The spectra presented here (7.14) represent the sum of individual spectra taken with a $2 \mathrm{~s}$ exposure time and an energy dispersion of $0.03 \mathrm{eV} / \mathrm{Channel}$. The individual spectra were offset from each other by $1-10 \mathrm{eV}$ by varying the drift tube energy and 
were then manually realigned during post-processing. This process allows efficient averaging of spectral noise and CCD artifacts as well as for long exposure times free from cumulative instrumental energy drift effects. The spectra were aligned to the $L_{3}$ edge $t_{2 g}$ peak according to the X-ray absorption near edge spectra (XANES) for $\mathrm{La}_{1-x} \mathrm{Sr}_{x} \mathrm{TiO}_{3}(0 \leq \mathrm{x} \leq 1)$ (Abbate et al., 1991), and then normalized to the highest peak $\left(L_{2}\right.$ edge $\mathrm{e}_{g}$ peak).

In figure 7.14 , four prominent peaks, $L_{2,3}$ edge $t_{2 g}$ and $\mathrm{e}_{g}$, for $x=0.002$ sample are gradually transformed to two very broad peaks with noticeable shoulders for $x=0.50$ thin film sample. The most significant figure is that all the peaks become broaden, especially for the sharp $t_{2 g}$ peaks shrinking to broad shoulders. Due to the large lifetime broadening, the change peak of the $t_{2 g}$ of $L_{2}$ edge is more significant than that of $L_{3}$ edge. The peak broadening can be explained from the crystal field multiplet effect: the original 7 possible final states for $\mathrm{Ti}^{4+} 2 p^{6} 3 d^{0} \rightarrow 2 p^{5} 3 d^{1}$ are replaced by 81 possible final states for $\mathrm{Ti}^{3+} 2 p^{6} 3 d^{1} \rightarrow 2 p^{5} 3 d^{2}$ in $\mathrm{O}_{h}$ symmetry (figure 7.15) although most of them have little intensity.

In addition to peak broadening, the energy splitting of $t_{2 g}$ and $e_{g}$ is also reduced as $\mathrm{Nb}$ content increases. Since the energy splitting of the $\mathrm{t}_{2 g}$ and $\mathrm{e}_{g}$ is related to the strength of the crystal field effect, the $\mathrm{Ti}^{3+}$ ion in octahedral symmetry feels a relative smaller coulombic interaction from surroundings. Compared to the $\mathrm{Ti}^{4+}$, the extra one electron of $\mathrm{Ti}^{3+}$ in outer $3 d$ orbital would screen more strongly the coulombic potential of surrounding ions and reduce the crystal field effect, giving a smaller splitting of $\mathrm{t}_{2 g}$ and $\mathrm{e}_{g}$. The $10 \mathrm{Dq}$ value of $\mathrm{Ti}^{3+}$ ion for the multiplets 


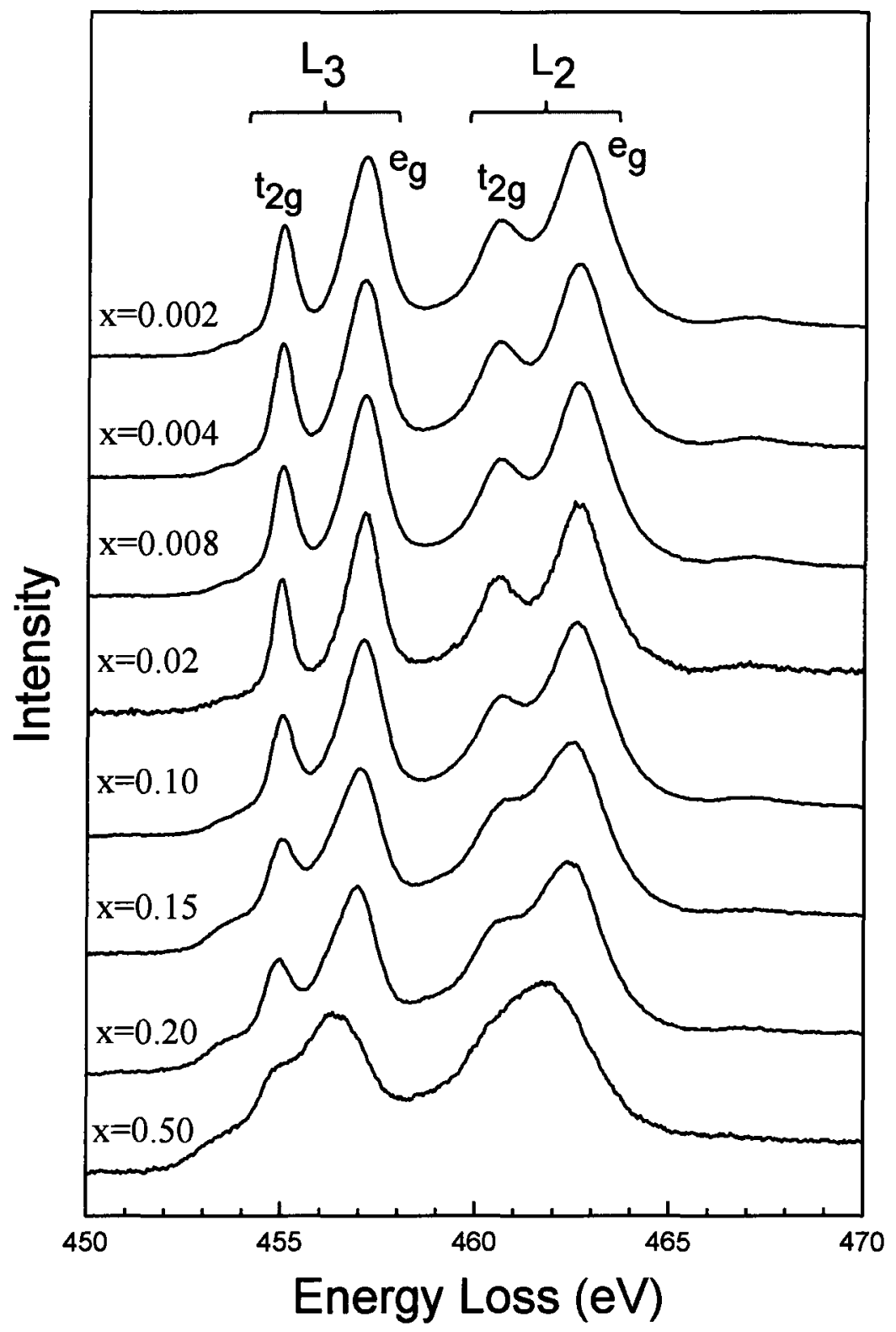

Figure 7.14: $\mathrm{Ti}-\mathrm{L}_{2,3}$ edge spectra for polycrystalline $\mathrm{BaTi}_{1-x} \mathrm{Nb}_{x} \mathrm{O}_{3}$ at $\mathrm{x}=0.002,0.004$, $0.008,0.02,0.10,0.15$, and 0.20 composition with thin film $\mathrm{BaTi}_{0.5} \mathrm{Nb}_{0.5} \mathrm{O}_{3}$ as comparison . The results demonstrate a gradual transformation in the oxidation state from $\mathrm{Ti}^{4+}$ to $\mathrm{Ti}^{3+}$ as niobium is substituted for titanium.

calculation here is $1.5 \mathrm{eV}$. 


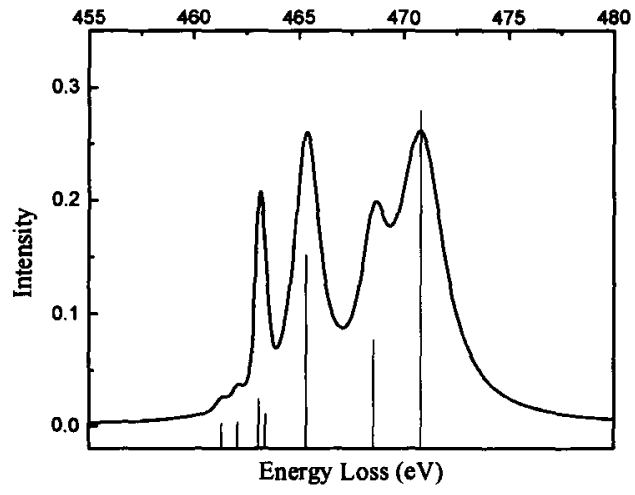

(a) $\mathrm{Ti}^{4+}$ in $\mathrm{O}_{h}$ symmetry

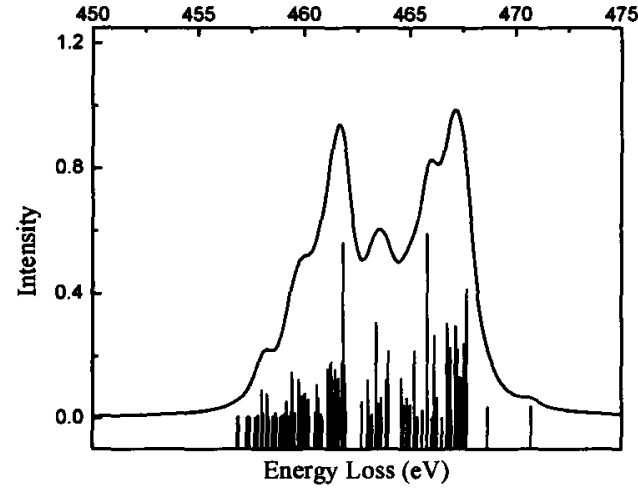

(b) $\mathrm{Ti}^{3+}$ in $\mathrm{O}_{h}$ symmetry

Figure 7.15: Multiplet calculation for (a) $\mathrm{Ti}^{4+}$ in $\mathrm{O}_{h}$ symmetry with 7 possible final states and (b) $\mathrm{Ti}^{3+}$ in $\mathrm{O}_{h}$ symmetry with 81 possible final states.

In ideal conditions, all the $\mathrm{Ti}^{4+}$ ions will reduce to $\mathrm{Ti}^{3+}$ at $x=0.50$ if we have $\mathrm{Nb}^{5+}$ doping. Since the $\mathrm{Ti}$ valence at one end member $\mathrm{BaTiO}_{3}$ corresponds exactly to $\mathrm{Ti}^{4+}$ in octahedral symmetry, and the $\mathrm{Nb}$ valence at another end member of the series stoichiometric $\mathrm{BaNbO}_{3}$ is $\mathrm{Nb}^{4+}$ in octahedral symmetry, there must be a valence mixture regions for $\mathrm{Ti}^{4+/ 3+}$ in $0<x<0.5$ and for $\mathrm{Nb}^{5+/ 4+}$ in $0.5<x<1$, while all the $\mathrm{Ti}$ remains $3+$ in $0.5<x<1$ and $\mathrm{Nb}$ remains $4+$ in $0<x<0.5$. The $\mathrm{Ti}^{4+/ 3+}$ valence mixture in $0<x \leq 0.2$ is very clear from the figure 7.14 as the $t_{2 g}$ peaks transform from a sharp peak to broad shoulders and the $e_{g}$ peaks broadens. The $\mathrm{Nb} L_{3}$ XANES experiments done by Taras Kolodiazhnyi (figure 7.16, unpublished) confirmed that the $\mathrm{Nb}$ ions remain in the valence of $5+$ in the same region of $0<x \leq 0.2$, but still cound not completely eliminat the possibility of the existance of $\mathrm{Nb}^{4+}$. In principle, we can also obtain the fractions of $\mathrm{Nb}^{5+}$ and $\mathrm{Nb}^{4+}$ by ELNES. However, the energy loss of $\mathrm{Nb} L_{2,3}$ edges are around 2350 $2500 \mathrm{eV}$ which is too far away from ZLP with bare intensity in ELNES; and the $M_{2,3}$ around $360-400 \mathrm{eV}$ are too broad to distinguish $\mathrm{Nb}^{5+}$ and $\mathrm{Nb}^{4+}$ due to the 
lifetime broadening. Therefore the $\mathrm{Nb}^{5+/ 4+}$ mixture is very difficult to observe in ELNES but in XANES, the $\mathrm{Nb} L_{2,3}$ is possible to be observed and distinguish $\mathrm{Nb}^{5+}$ and $\mathrm{Nb}^{4+}$. However, we don't have any high quality polycrystalline samples with $x>0.5$, and the expected $\mathrm{Nb}^{5+/ 4+}$ mixture region havn't been reported.

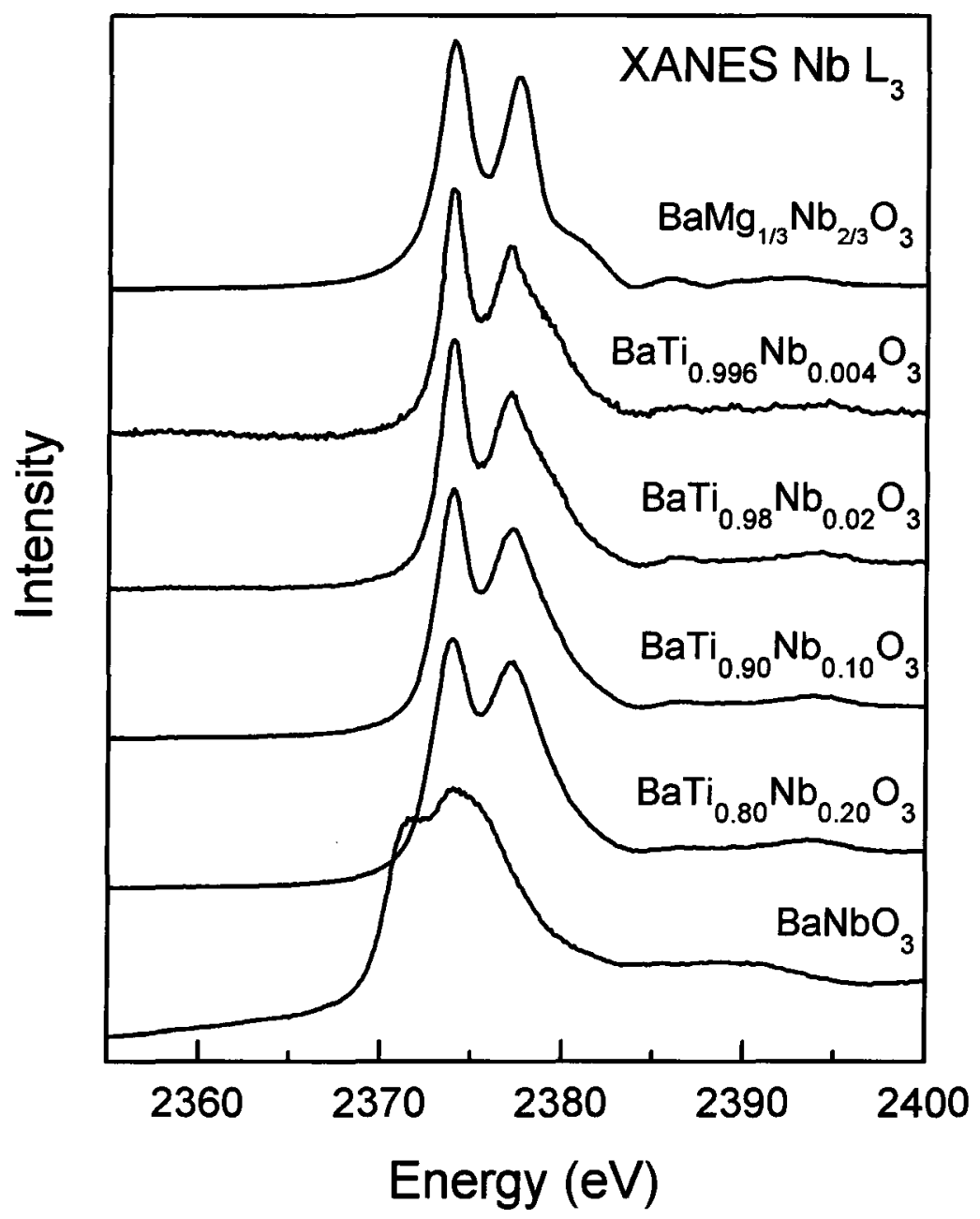

Figure 7.16: $\mathrm{Nb}-L_{3}$ edge XANES of polycrystalline $\mathrm{BaTi}_{1-x} \mathrm{Nb}_{x} \mathrm{O}_{3}$ at $\mathrm{x}=0.004,0.02,0.10$ and 0.20 composition with two reference spectra: $\mathrm{BaMg}_{2 / 3} \mathrm{Nb}_{1 / 3} \mathrm{O}_{3}$ for $\mathrm{Nb}^{5+}$ and $\mathrm{BaNbO}_{3}$ for $\mathrm{Nb}^{4+}$. All the $\mathrm{Nb}-L_{3}$ edges for $\mathrm{BaTi}_{1-x} \mathrm{Nb}_{x} \mathrm{O}_{3}$ samples have a similar shape to the one in the $\mathrm{BaMg}_{1 / 3} \mathrm{Nb}_{2 / 3} \mathrm{O}_{3}$ and exactly the same onset position, confirming they are all $\mathrm{Nb}^{5+}$. (unpublished, by courtesy of Taras Kolodiazhnyi) 


\subsubsection{O-K edges}

Generally the O-K edges are more readily interpreted based on the electronic and atomic structure of the compound than $L_{2,3}$ edges of transition metals (Kurata et al., 1993). Unlike the Multiplet effects which are dominated $L_{2,3}$ edges for the transition metals, $K$ edge spectra can be easily explained by the single electron approximation due to the strongly electronic screening effect preventing the hole in the initial state to interact with the electron in the final state. Thus this allows the interpretation of spectra based on the powerful molecular orbital picture and the unoccupied density of states.

In figure 7.17 , the $\mathrm{O}-\mathrm{K}$ edge electron energy loss spectra from polycrystalline $\mathrm{BaTi}_{1-x} \mathrm{Nb}_{x} \mathrm{O}_{3}$ samples (black color curve, $x<0.5$ ) is given with the $\mathrm{O}-\mathrm{K}$ edges from thin film $\mathrm{BaTi}_{1-x} \mathrm{Nb}_{x} \mathrm{O}_{3}$ samples (dark blue color curve, $x \geq 0.5$ ). For each spectrum, 7 peaks are indicated by $a, b, c, d, e, f$ and $g$; and all spectra are aligned

to the peak $f$. As the $\mathrm{Nb}$ content increases, a sharp peak $a$ systematically shifts to the high energy part and the farthest energy separation of peak $a$ between $x=0.002$ and $x=1.0$ is $1.2 \mathrm{eV}$; the peak $b$ as a shoulder $2.2 \mathrm{eV}$ after peak $a$ and gradually becomes weaker and completely disappears at $x=1.0$; the total intensities of peak $c$ and $d$ increase as compared to peak $a$, and the intensity of peak $c$ increases more rapidly than peak $d$, changing the shape of local intensity from $x<0.01$ to $\geq 0.5$; and the positions of peak $e, f$ and $g$ are quite stable for the whole series.

The interpretation of the O- $K$ edge was carried out by calculating the partial density of states for the two end member $\mathrm{BaTiO}_{3}$ and $\mathrm{BaNbO}_{3}$ via WIEN2k pro- 


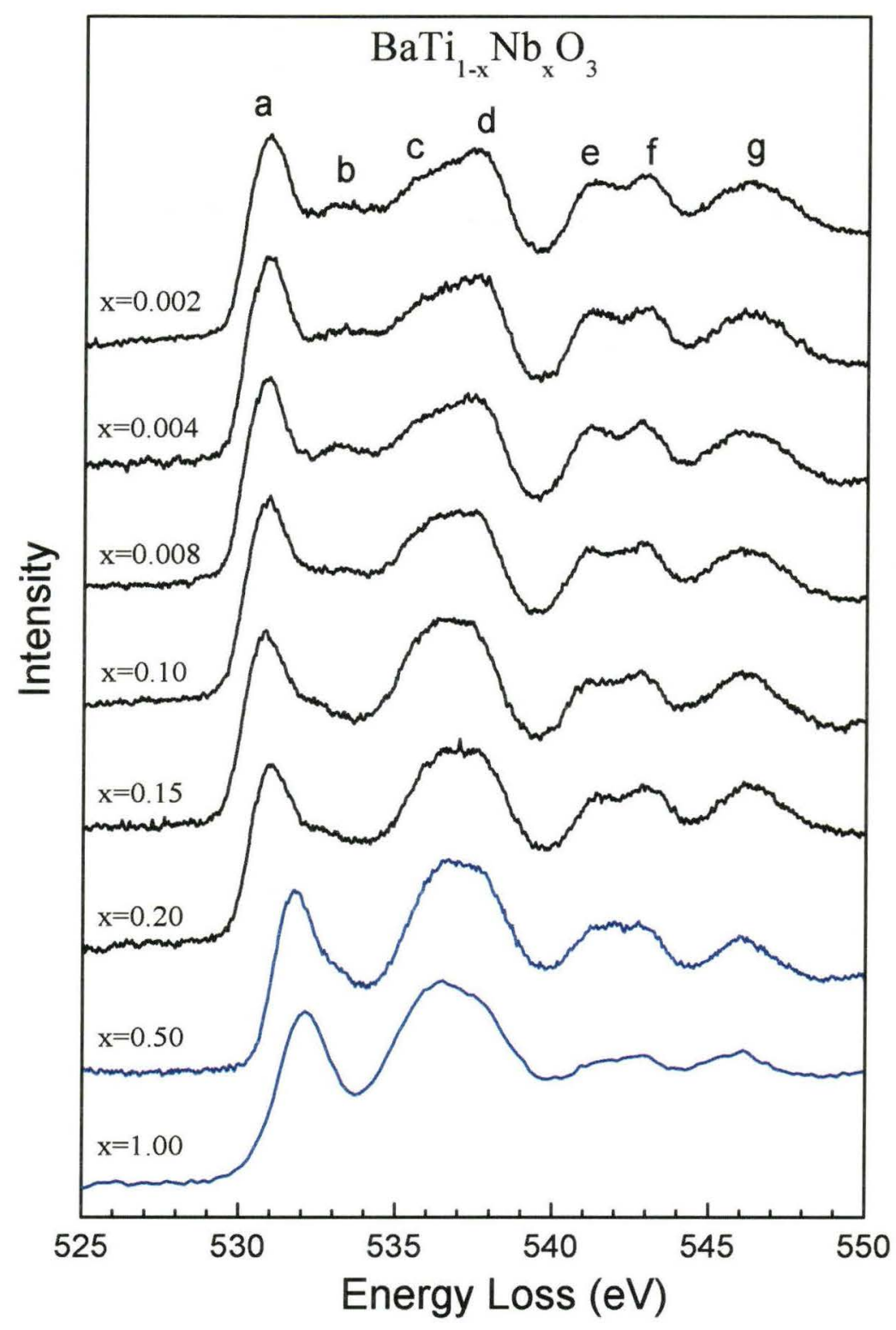

Figure 7.17: $\mathrm{O}-K$ edge ELNES of $\mathrm{BaTi}_{1-x} \mathrm{Nb}_{x} \mathrm{O}_{3}$ of polycrystalline $\mathrm{BaTi}_{1-x} \mathrm{Nb}_{x} \mathrm{O}_{3}$ at $x=0.002,0.004,0.008,0.10,0.15$, and 0.20 compositions (black color) with thin film $\mathrm{BaTi}_{1-x} \mathrm{Nb}_{x} \mathrm{O}_{3}$ at $x=0.50,0.75$ and 1.0 composition (dark blue color).

gram (see section 4.1 on page 56). The calculation was under the framework of local density approximation (LDA) and generalized gradient approximation (GGA). The structural information of $\mathrm{BaTiO}_{3}$ and $\mathrm{BaNbO}_{3}$ phase for calculation are listed in the 
table 7.3.

Table 7.3: Unit cell and atomic parameters for tetragonal $\mathrm{BaTiO}_{3}$ and cubic $\mathrm{BaNbO}_{3}$

\begin{tabular}{|c|c|c|c|c|c|c|}
\hline Compound & \multicolumn{3}{|c|}{$\mathrm{BaTiO}_{3}{ }^{a}$} & \multicolumn{3}{|c|}{$\mathrm{BaNbO}_{3}{ }^{b}$} \\
\hline Space group & \multicolumn{3}{|c|}{ P4mm (99) } & \multicolumn{3}{|c|}{$\operatorname{Pm} 3 \mathrm{~m}(225)$} \\
\hline $\mathrm{a}(\AA)$ & \multicolumn{3}{|c|}{3.9945} & \multicolumn{3}{|c|}{4.0931} \\
\hline$b(\AA)$ & \multicolumn{3}{|l|}{-} & \multicolumn{3}{|l|}{-} \\
\hline$c(\AA)$ & \multicolumn{3}{|c|}{4.0335} & \multicolumn{3}{|l|}{ - } \\
\hline Atom & \multicolumn{3}{|c|}{ reduced coord. $(x, y, z)$} & \multicolumn{3}{|c|}{ reduced coord. $(x, y, z)$} \\
\hline $\mathrm{Ba}$ & 0.00 & 0.00 & 0.00 & 0.00 & 0.00 & 0.00 \\
\hline $\mathrm{Ti}$ & 0.50 & 0.50 & 0.514 & & & \\
\hline $\mathrm{Nb}$ & & & & 0.50 & 0.50 & 0.50 \\
\hline $\mathrm{O}(1)$ & 0.50 & 0.50 & 0.975 & 0.50 & 0.50 & 0.00 \\
\hline $\mathrm{O}(2)$ & 0.00 & 0.50 & 0.488 & & & \\
\hline
\end{tabular}

The atomic projected partial DOS (PDOS) of $\mathrm{BaTiO}_{3}$ and $\mathrm{BaNbO}_{3}$ are shown in figure 7.18. From figure 7.18(a), It is clear that there is an energy gap between the occupied $\mathrm{O} 2 p$ states and the unoccupied $\mathrm{Ti} 3 d$ states, which means that the $\mathrm{BaTiO}_{3}$ is a band insulator with $\mathrm{Ti} 3 d^{0}$ electron configuration. The value of the energy gap is about $1.88 \mathrm{eV}$, which is small in respect to the experimental value of about $3.2 \mathrm{eV}$. The reason for such a disagreement is the well-known shortcoming of the LDA for semiconductors and insulators (Jones \& Gunnarsson, 1989). From figure 7.18(b), we note that the Fermi level moves into conduction band demonstrating a metallic behavior for $\mathrm{BaNbO}_{3}$. Since the Fermi level is not the same as in $\mathrm{BaTiO}_{3}$ and $\mathrm{BaNbO}_{3}$, we can not simply compare their PDOS according to the Fermi level. Here a reasonable scheme to compare the two PDOS is based on the $\mathrm{Ba} 5 d$ onset position since the $\mathrm{Ba}$ energy level should not change much in both $\mathrm{BaTiO}_{3}$ and 


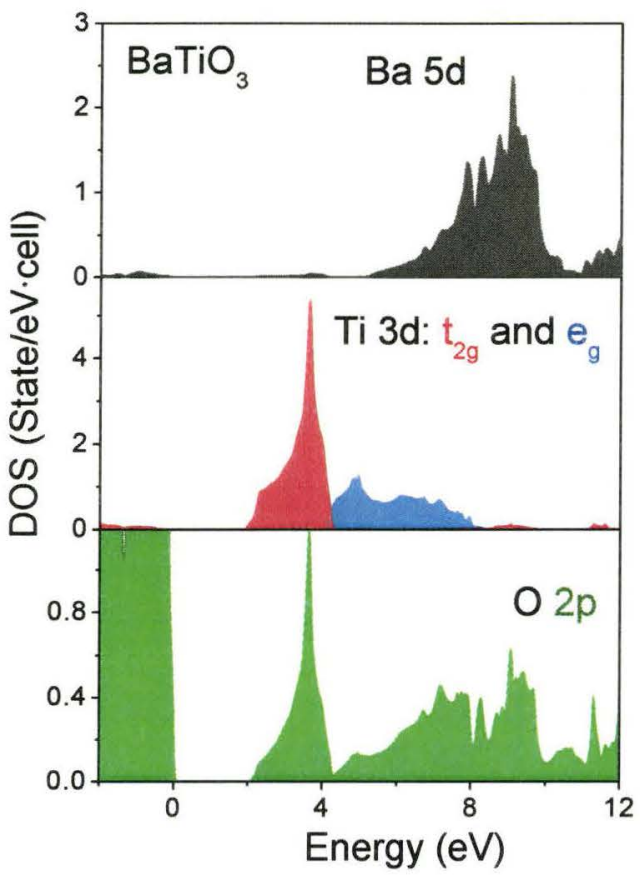

(a) PDOS of $\mathrm{BaTiO}_{3}$

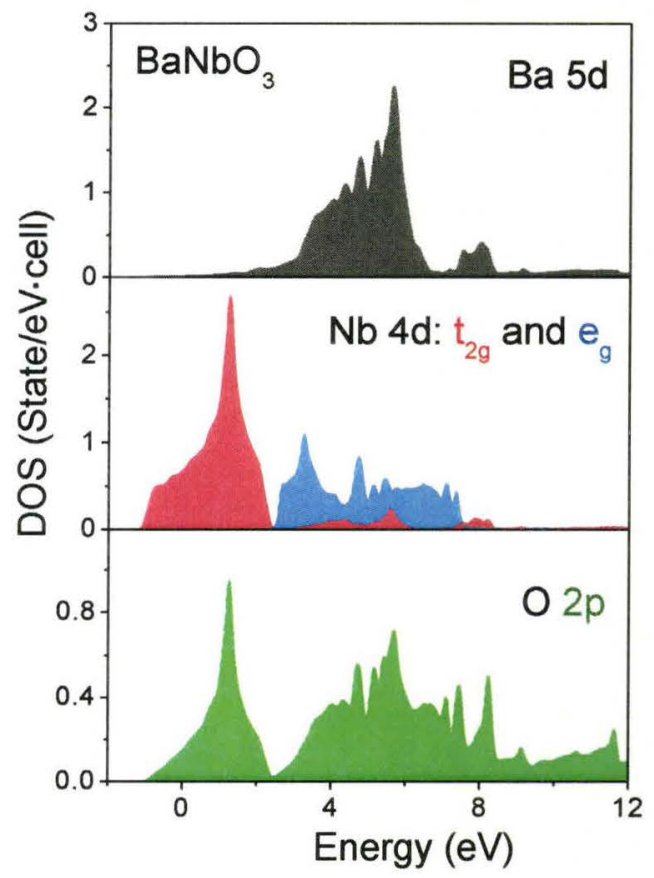

(b) $\mathrm{PDOS}$ of $\mathrm{BaNbO}_{3}$

Figure 7.18: Site projected PDOS of (a) $\mathrm{BaTiO}_{3}$ and (b) $\mathrm{BaNbO}_{3}$, Fermi level was set to zero point.

$\mathrm{BaNbO}_{3}$ compounds. The narrow $\mathrm{Ti}_{2 g}$ band representing a $\pi^{\star}$ anti-bonding is followed by a broad $\mathrm{Ti}_{g}$ band representing a $\sigma^{\star}$ anti-bond. Most of the $\mathrm{Ba} 5 d$ and Ti $3 d$ bands are not in the same energy range. However for the $\mathrm{BaNbO}_{3}$, the $\mathrm{Nb} 4 d$ band is relatively broadener than the Ti $3 d$ band due to the more stronger electron screening effect, and the $\mathrm{Nb} 4 d \mathrm{t}_{2 g}$ and $\mathrm{e}_{g}$ levels have also systematically moved to higher energy part as compared to the counterparts of the Ti $3 d$ band based on the $\mathrm{Ba} 5 d$ position. The $\mathrm{Nb} 4 d \mathrm{e}_{g}$ band does significantly overlap with $\mathrm{Ba} 5 d$ in the same energy range (figure 7.18(b)). Therefore for the low $\mathrm{Nb}$ doped $\mathrm{BaTiO}_{3}$ samples (figure 7.17), peak $a$ of the O-K edge mainly represents the hybridization with the $\mathrm{Ti}_{2 g}$ band, the peak $b$ represents the hybridization with the $\mathrm{Ti}_{g}$ band 
and peak $c \& d$ are the hybridization with the $\mathrm{Ba} 5 d$ band. When the $\mathrm{Nb}$ content increases, the contribution of $\mathrm{Ti} 3 \mathrm{~d}$ band will decrease while the contribution of $\mathrm{Nb}$ appears to increase. As a consequence of the high $\mathrm{Nb}$ doped $\mathrm{BaTiO}_{3}$ samples, not only the $\mathrm{Ti} 3 d \mathrm{t}_{2 g}$ figure replaces by the $\mathrm{Nb} 4 d \mathrm{t}_{2 g}$ figure, demonstrated by a chemical shift of peak $a$ in figure 7.17 moving to higher energy part; but also the $\mathrm{Ti}$ $3 d \mathrm{e}_{g}$ replaces by the $\mathrm{Nb} 4 d t_{2 g}$ figure, demonstrated by the peak $b$ disappearance and peak $c$ increasing, eventually changing the relatively intensity of peak $c$ and $d$. The $1.2 \mathrm{eV}$ chemical shift of peak $a$ between $x=0.002$ and $x=1.0$ samples agrees well with the energy shift of the Ti $3 d \mathrm{t}_{2 g}$ and $\mathrm{Nb} 4 d \mathrm{t}_{2 g}$ band.

The calculation of the O- $K$ edge ELNES is still under study by WIEN2k package using LDA+U method with core hole effect, and will not be included in this work. The lorentzian broadening part of the broadening program for ELNES calculation in WIEN2k package, however, is not correct. We listed a proper MATLAB script for the lorentzian broadening for ELNES calculation in appendix C.

\subsection{Resistivity measurement}

The resistivities of the films produced were strongly dependent upon both the oxygen and niobium content. All films exposed to 380 Torr of oxygen were insulating at room temperature. Films cooled in vacuum became increasingly conductive as the niobium content was increased. Accompanying this increased conductivity was a film color transformation from transparent to a dark blue-green. Figure 7.19 shows the temperature dependent resistivity as a function of the niobium content. The 
curve shows a semiconducting to metallic transition near $x=0.20$, consistent with the thin film work of (Liu et al., 2005), but in contrast to most other reports. The metallic samples show excellent conductivity with values that are in line with other well established oxide conductors. Also of note is that, despite the lower niobium content, the $x=0.002$ film shows a lower resistivity than the $x=0.02$ sample. The reason for this effort will be explained later by the Hall effect measurements using van de Pauw method (see section 7.10 on page 123).

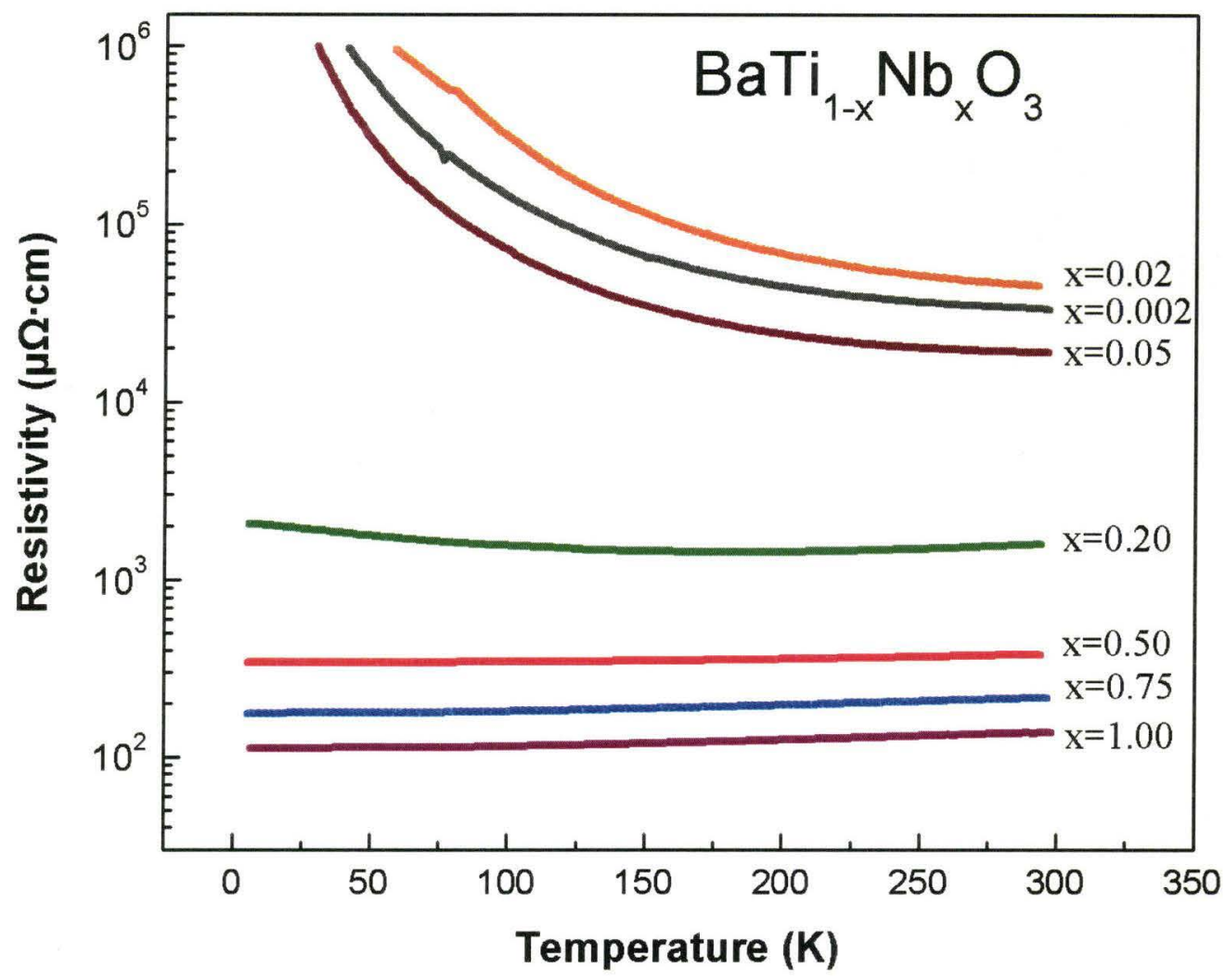

Figure 7.19: The temperature dependent resistivity of $\mathrm{BaTi}_{1-x} \mathrm{Nb}_{x} \mathrm{O}_{3}$ as a function of $\mathrm{Nb}$ concentration. With increasing niobium content the material becomes progressively more conducting with the semiconductor to metal transition occurring near $x=0.2$. Note that the film with $x=0.02$ is more resistive than the one with $x=0.002$. Measurements were performed using the Van der Pauw technique. 


\subsection{I-V curve}

We used gold for metal contacts in the resistivity measurements. Since the gold has a relatively large work function, Schottky barrier has been found in the interface of many polycrystal semiconductors and gold contacts, resulting a rectifying characteristics. However, this effect is not always present. The existence of a Schottky barrier does depend on the surface electronic state and, of course, the different orientation of the crystals.

In order to determine whether the Schottky barrier causes possible artifacts, the $I-V$ curve measurements were performed. Ohmic contacts were confirmed by the linear $I-V$ curves from insulating part $(x=0.002$ and $x=0.02)$ to metallic part $(x=0.50)$ at room temperature (shown in figure 7.20$)$. Since there is no Shottky barrier, our Hall measurements are expected to be reliable. As the understanding of the complex nature of Schottky barrier would require investigations of the surface electronic states between deposited gold contacts and [001] orientated $\mathrm{BaTi}_{1-x} \mathrm{Nb}_{x} \mathrm{O}_{3}$ films, we will not explore these effects further since this discussion is beyond the scope of this thesis.

\subsection{Hall coefficient measurement}

In order to understand the nature of charge carries and their mobility, room temperature Hall coefficient measurements were carried out with ACCENT, HL5500 PC 


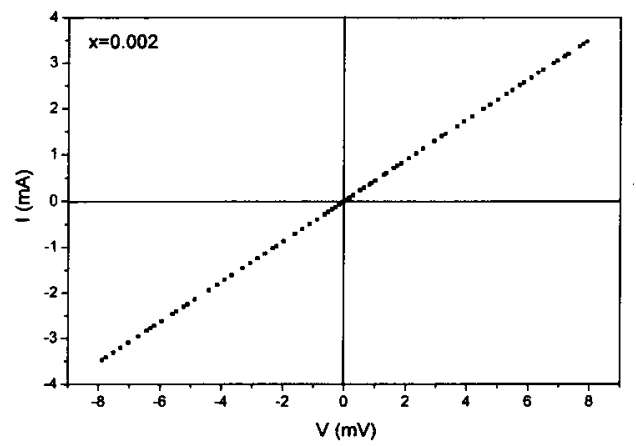

(a) $I-V$ curve of $\mathrm{x}=0.002$ film (small range of $\mathrm{V}$ )

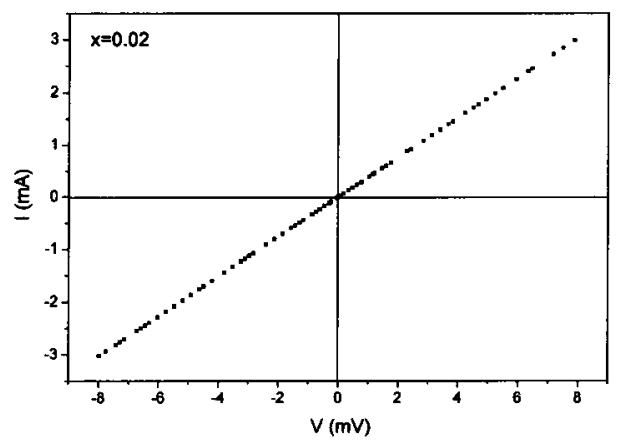

(c) I-V curve of $\mathrm{x}=0.02$ film (small range of $\mathrm{V}$ )

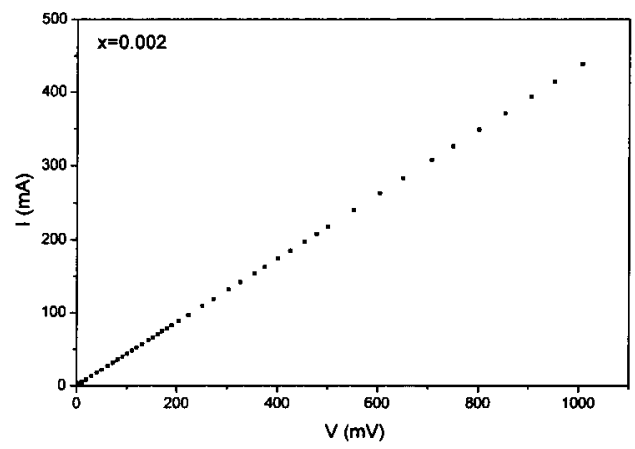

(b) $I-V$ curve of $\mathrm{x}=0.002$ film (large range of $\mathrm{V}$ )

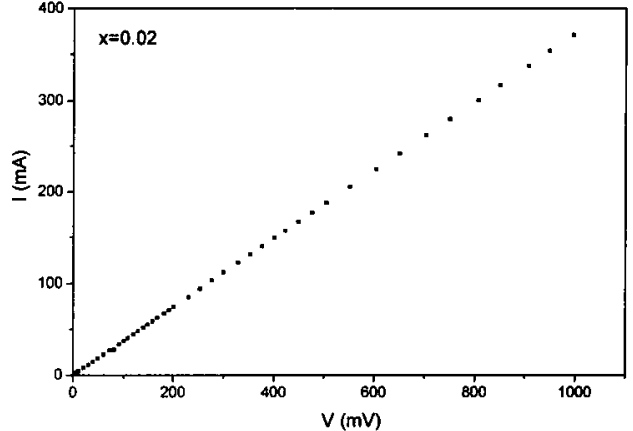

(d) $I-V$ curve of $\mathrm{x}=0.02$ film (large range of $\mathrm{V}$ )

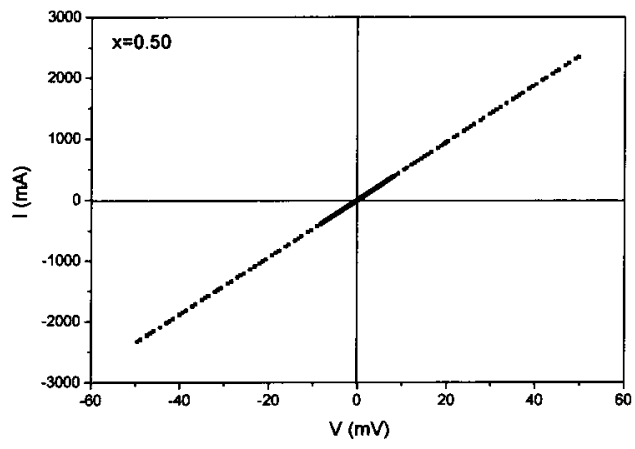

(e) $I-V$ curve of $\mathrm{x}=0.50$ film

Figure 7.20: Linear $I-V$ curves from insulatting part ( $x=0.002$ and $x=0.02$ ) to metallic part $(x=0.50)$ at room temperature confirm the presence of Ohmic contacts for all $\mathrm{BaTi}_{1-x} \mathrm{Nb}_{x} \mathrm{O}_{3}$ films 
Hall Effect Measurement System. The measurements were performed under DC mode with magnetic field of 0.32 Tesla. The results are listed in the Table 7.4. The values are similar to the results obtained by Liu et al. (2005) (see table 2.2 on page 39).

Table 7.4: Hall effect measurements of $\mathrm{BaTi}_{1-x} \mathrm{Nb}_{x} \mathrm{O}_{3}$

\begin{tabular}{lccccc}
\hline Sample & $\rho_{R T}(\Omega \cdot \mathrm{cm})$ & Sheet Hall Coeff. $\left(\mathrm{m}^{2} / \mathrm{C}\right)$ & $\mu_{H}\left(\mathrm{~cm}^{2} / \mathrm{V} \cdot \mathrm{s}\right)$ & type & $\mathrm{n}\left(/ \mathrm{cm}^{3}\right)$ \\
\hline $\mathrm{x}=0.002$ & $3.40 \times 10^{-2}$ & $+1.09 \times 10^{-1}$ & 0.965 & $\mathrm{p}$ & $1.90 \times 10^{20}$ \\
$\mathrm{x}=0.02$ & $4.60 \times 10^{-2}$ & $-1.21 \times 10^{-1}$ & 0.789 & $\mathrm{n}$ & $1.72 \times 10^{20}$ \\
$\mathrm{x}=0.05$ & $1.95 \times 10^{-2}$ & $-1.34 \times 10^{-1}$ & 2.07 & $\mathrm{n}$ & $1.55 \times 10^{20}$ \\
$\mathrm{x}=0.20$ & $1.73 \times 10^{-3}$ & $-4.05 \times 10^{-2}$ & 7.01 & $\mathrm{n}$ & $5.14 \times 10^{20}$ \\
$\mathrm{x}=0.50$ & $4.04 \times 10^{-4}$ & $-2.03 \times 10^{-2}$ & 15.1 & $\mathrm{n}$ & $1.02 \times 10^{21}$ \\
$\mathrm{x}=0.75$ & $2.18 \times 10^{-4}$ & $-1.56 \times 10^{-3}$ & 2.15 & $\mathrm{n}$ & $1.33 \times 10^{22}$ \\
$\mathrm{x}=1.00$ & $1.40 \times 10^{-4}$ & $-1.23 \times 10^{-3}$ & 2.65 & $\mathrm{n}$ & $1.69 \times 10^{22}$ \\
\hline
\end{tabular}

The Hall effect measurements indicate that there is a reversal in the type of the charge carriers from p-type for $x=0.002$ to $\mathrm{n}$-type for all the after samples. The sign of the Hall coefficient (table 7.4) is positive for $x=0.002$ and negative for all other $\mathrm{Nb}$ concentrations, indicating that the charge carries are holes at $\mathrm{x}=0.002$ and become electrons after $x=0.02$. The compensation point is between $x=0.002$ and 0.02. The change of charge carriers is also found in $\mathrm{BaTi}_{1-x} \mathrm{Nb}_{x} \mathrm{O}_{3}$ ceramic sample between 250-500 ppm (Chang et al., 1999). This different compensation point can arise from the different initial density of cation vacancies due to different sample preparation procedure. The carrier density $n$ can also be calculated from the sheet Hall coefficient (equation 3.10 on page 54). We also notice an increased value of Hall mobility for the $x=0.5$ sample which is in the same order of the $\mathrm{Ba}_{1-y} \mathrm{Sr}_{y} \mathrm{Ti}_{0.5} \mathrm{Nb}_{0.5} \mathrm{O}_{3}$ samples (see table 9.3 on page 173). However, the real nature is not clear. 
This change in charge carries type can explain the previous resistivity results (the resisitivity of $x=0.002$ sample is lower than the resistivity of the $x=0.02$ sample) between $x=0.002$ and $x=0.02$ samples. Pure $\mathrm{BaTiO}_{3}$ is an insulator with a band gap of $3.2 \mathrm{eV}$ but normally it becomes p-type semiconductor due to p-type defect, such as cation vacancies, and the main charge carriers are holes with a certain density which is depend on the synthesis condition. When $\mathrm{Nb}$ ions, the donors, are incorporated into the $\mathrm{BaTiO}_{3}$ lattice, the p-type defect will be compensated gradually and the initial density of holes will decrease, resulting in a decrease of the total charge carrier density. As a consequence, the resistivity will increase as $\mathrm{Nb}$ doping when holes are still the major charge carriers. At a certain $\mathrm{Nb}$ doping concentration, so-called compensation point, all holes will be completely compensated and the resistivity will reach a maximum. After that point, the electrons will become the major charge carriers and the resistivity will decrease as $\mathrm{Nb}$ doping increase. This evolution of charge carries type change is schematically illustrated in figure 7.21 .

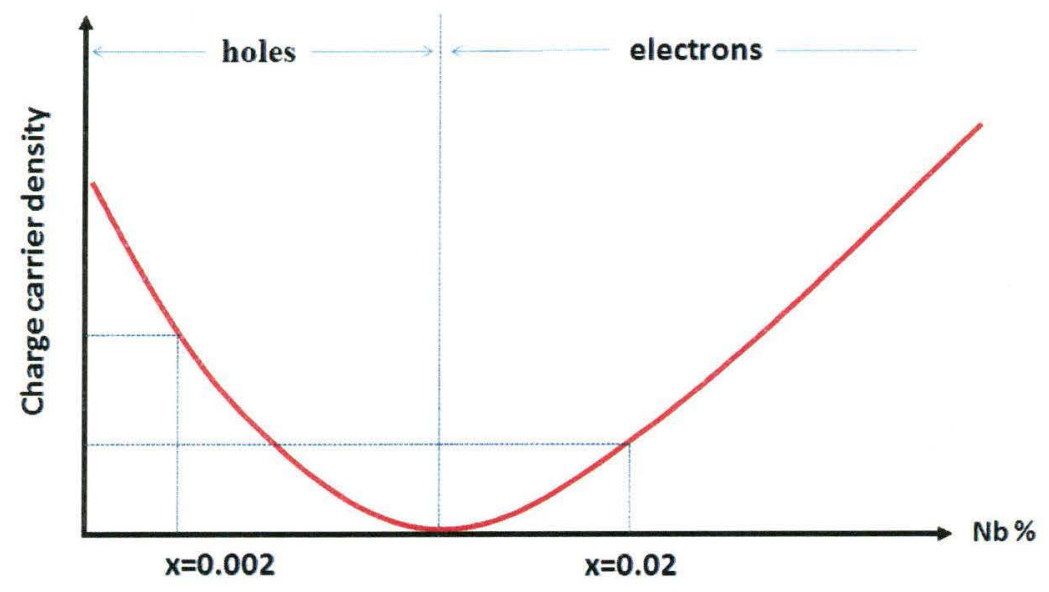

Figure 7.21: A schematically illustration of charge carries type and densities change 
The carrier charge density as a function of $\mathrm{Nb}$ contents is also plotted in figure 7.22. When the $\mathrm{Nb}$ content is less than 0.50 , the charge density increases almost linearly. However, a significant increase of charge density occurs when the $\mathrm{Nb}$ content is larger than 0.50 . The reason for this effect can be explained by the EELS results: when the $\mathrm{Nb}$ content is less than 0.50 , the extra electron provided by $\mathrm{Nb}$ will be trapped on the Ti site, resulting in not only the Ti valence change from 4+ to 3+ but also a slow increase in the charge density; when the $\mathrm{Nb}$ content exceeds 0.50 , all $\mathrm{Ti}$ atoms are converted to $3+$ and there is no more Ti site that traps the extra electrons, resulting a free-electron-like nature for the extra electrons and therefore a significant increase of the charge density.

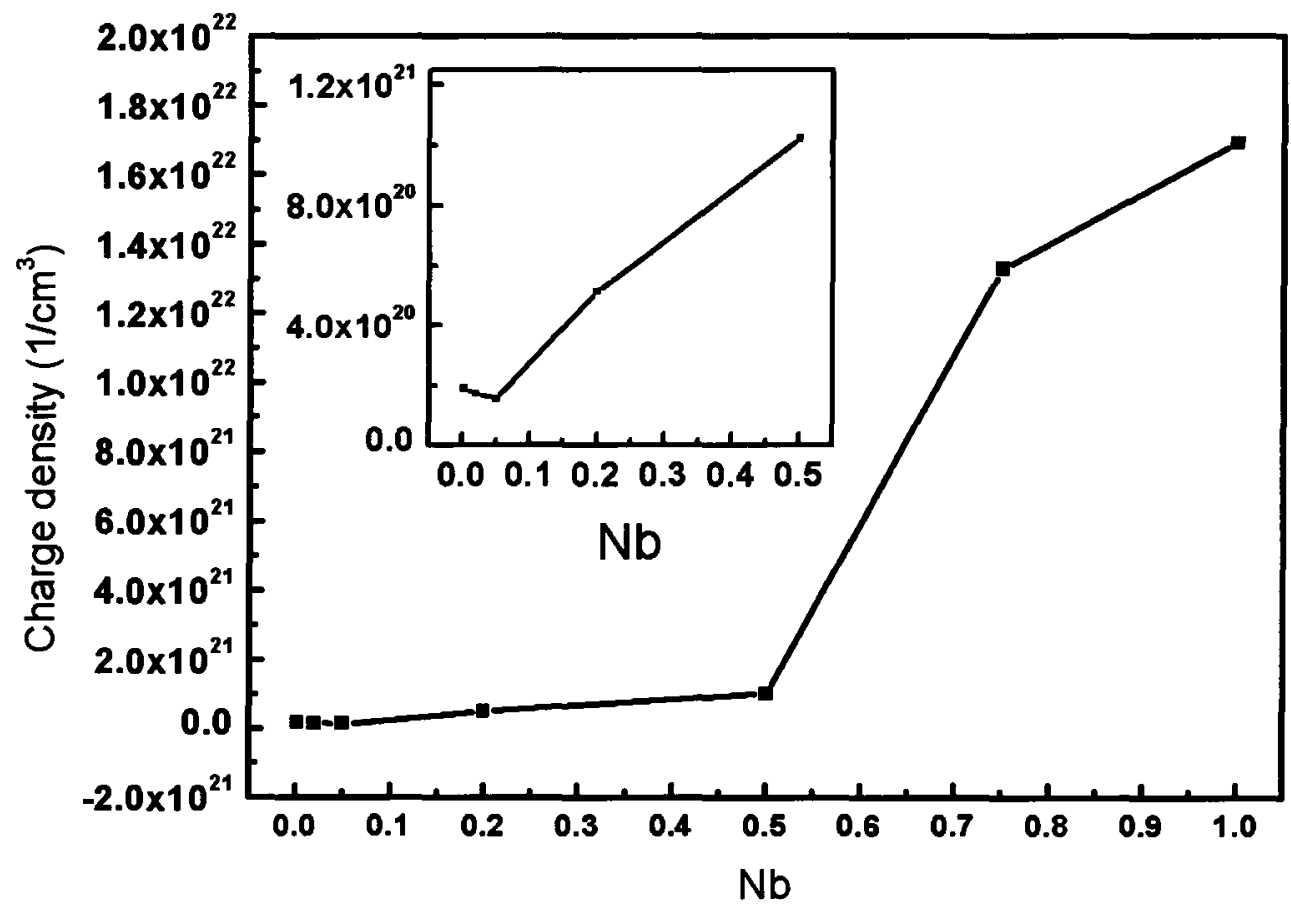

Figure 7.22: Plot of the charge density as a function of $\mathrm{Nb}$ content 


\subsection{A possible 2D Percolation phenomenon}

A first experimental report of a percolation transition in a 2D electron system was published 2005 in a n-GaAs system (Das Sarma et al., 2005), while the earlier percolation phenomena were focused on 2D hole systems. The authors (Das Sarma et al., 2005) fitted their conductivity by equation 7.1, where $a$ is a constant, $n$ is the charge density, $b$ is the critical density and $c$ is the conductivity exponent index. They claimed that their measured conductivity exponent index of $c$ is around $1.4-1.5$ which is close to the 2D percolation theoretical value of $4 / 3$ at rather low temperature $(<0.5 \mathrm{~K})$ and support a disorder-driven crossover phenomenon to explain the metal-insulator transition.

$$
\sigma=a(n-b)^{c}
$$

The basic picture of the percolation transition for a $2 \mathrm{D}$ metal-insulator transition is highly physically motivated. As the charge density decreases, the electrostatic screening effect will become weaker and unable to screen the disorder potential, resulting in a random "hill-and valley" potential scenario with charge carriers filling in the potential valley. This is different from the high carrier density homogeneous situation. Once the disorder potential hills are numerous enough to prevent percolating conducting paths to span the $2 \mathrm{D}$ system, the $2 \mathrm{D}$ metal-insulator transition transition will take place. The system is insulating below certain critical charge density and after that point, becomes metallic. The presence of random charged impurities in the system could arise the disorder potential and then favor the 2D percolation picture. Triggered by this interesting phenomenon, we plotted the con- 
ductivity variation with different $\mathrm{Nb}$ concentrations at four different temperature $(50 \mathrm{~K}, 100 \mathrm{~K}, 200 \mathrm{~K}$ and $290 \mathrm{~K}$ ) and then fitted by the power law as equation 7.1 (figure 7.23). All fitted parameters are listed in the table 7.5.

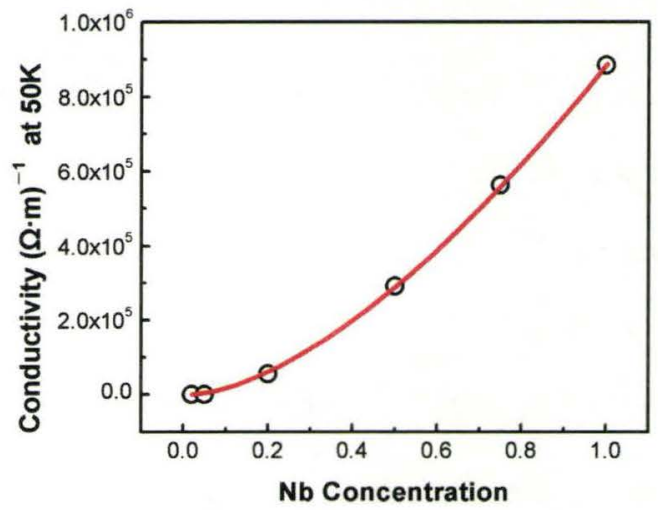

(a) $50 \mathrm{~K}$

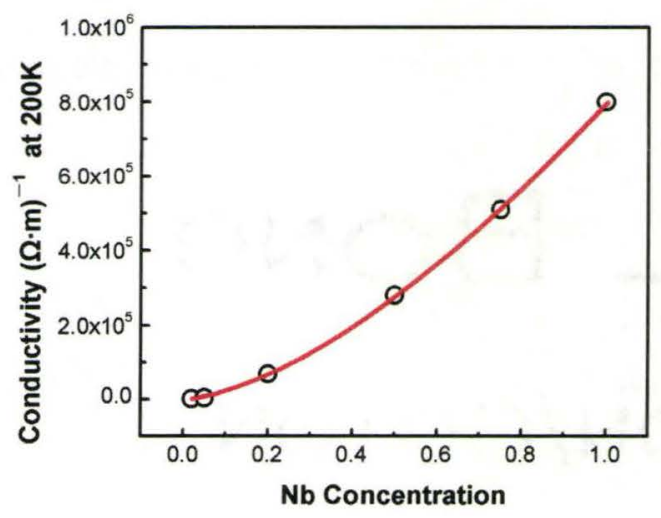

(c) $200 \mathrm{~K}$

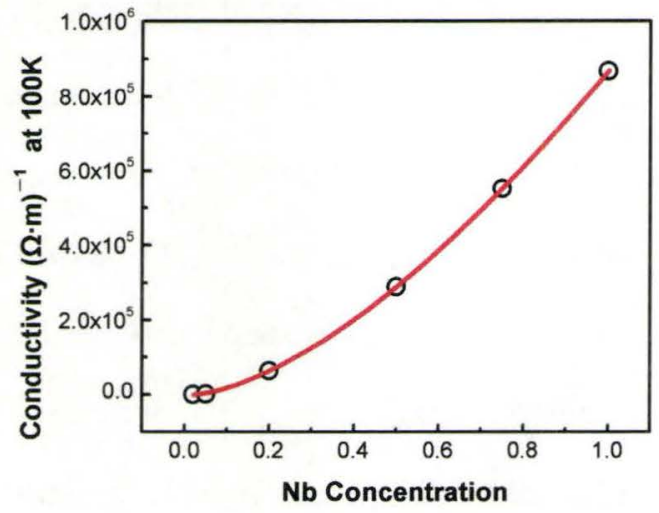

(b) $100 \mathrm{~K}$

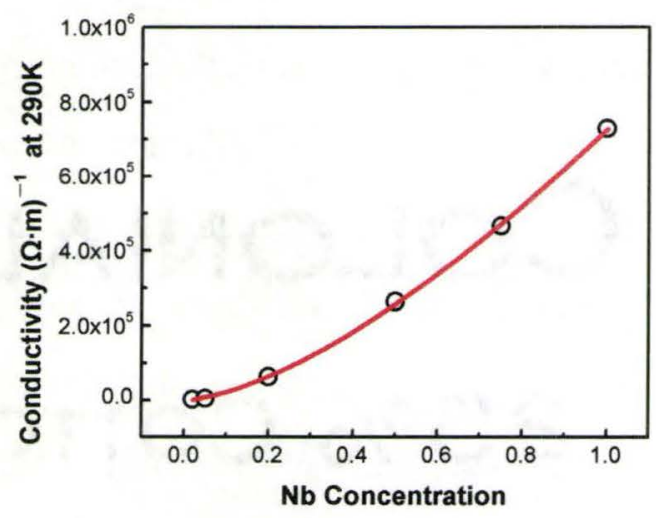

(d) $290 \mathrm{~K}$

Figure 7.23: Conductivity variation with different $\mathrm{Nb}$ concentrations at (a) $50 \mathrm{~K}$, (b) 100 $\mathrm{K}$, (c) $200 \mathrm{~K}$ and (d) $290 \mathrm{~K}$

For our experiment, the conductivity exponents $c$ are around 1.48-1.57 at all sampled temperature, which are not far from the theoretical 2D percolation expo- 
Table 7.5: Fitting parameters for 2D percolation phenomena by equation 7.1

\begin{tabular}{cccc}
\hline Sampled temperature & $\mathrm{a}$ & $\mathrm{b}$ & $\mathrm{c}$ \\
\hline $50 \mathrm{~K}$ & $(9.2 \pm 0.3) \times 10^{5}$ & $0.020 \pm 0.018$ & $1.57 \pm 0.05$ \\
$100 \mathrm{~K}$ & $(8.9 \pm 0.1) \times 10^{5}$ & $0.020 \pm 0.006$ & $1.54 \pm 0.02$ \\
$200 \mathrm{~K}$ & $(8.0 \pm 0.2) \times 10^{5}$ & $0.003 \pm 0.014$ & $1.52 \pm 0.04$ \\
$290 \mathrm{~K}$ & $(7.3 \pm 0.2) \times 10^{5}$ & $0.006 \pm 0.022$ & $1.48 \pm 0.06$ \\
\hline
\end{tabular}

nent value of $4 / 3$, and similar to experimental $2 \mathrm{D}$ percolation value of 1.4 found by Das Sarma et al. (2005). On the other hand, the electron conduction in thin films would also like to favor the $2 \mathrm{D}$ picture rather than $3 \mathrm{D}$ picture due to the real dimension limitation. It seems that our electron doping systems support the possible 2D percolation picture but this interpretation still need to be investigated cautiously and more work is necessary. The critical charge density, which is parameter $b$ in the table 7.5 , is far below the critical $\mathrm{Nb}$ content $(\sim 20 \%)$ of the metal-insulator transition we can see based on the sign of $d \sigma / d T$. One reason could be that the sign of $d \sigma / d T$ is not a good indicator for the critical density (Das Sarma et al., 2005) because the effective metallic phase can exhibit an insulating sign down to rather low temperature (Das Sarma \& Hwang, 2004), and therefore any determination of the critical charge density based only on the sign of $d \sigma / d T$ will obtain a larger value than the real one (Das Sarma et al., 2005). 


\subsection{Magnetic properties and possible superconduct- ing phase}

Magnetic properties were measured by PPMS in AC transmission mode that is much sensitive than DC mode, with an oscillation magnetic field of $4 \mathrm{Oe}$ at 1000 Hz. Since we cannot remove the thin films from the substrate, here the experimental measurement of the magnetic properties (spin moment) of thin films include the contribution from the substrate which we need to subtract later. In figure 7.24, we presented the results from a bare $\mathrm{MgAl}_{2} \mathrm{O}_{4}$ substrate, $x=0.50 \mathrm{Nb}$-doped ceramic target, and $x=0,0.05,0.20$ and $0.50 \mathrm{Nb}$-doped $\mathrm{BaTiO}_{3}$ thin films. The bare $\mathrm{MgAl}_{2} \mathrm{O}_{4}$ substrate result will be used as a standard to obtain the spin moment contribution from the substrate. The result from $x=0.50 \mathrm{Nb}$-doped ceramic target will be used as a reference to compare it with the result from the corresponding thin film, while the result from $\mathrm{BaTiO}_{3}$ thin film will be used as a reference to compare it with other $\mathrm{Nb}$-doped thin films,.

From figure 7.24 (a) we can see that $\mathrm{MgAl}_{2} \mathrm{O}_{4}$ is a diamagnetic material, but the other samples have abnormal increases at high temperature range (from $220 \mathrm{~K}-300$ $\mathrm{K})$. These abnormal increase of spin moments were also found by Kolodiazhnyi \& Wimbush (2006), who use ceramic polycrystalline materials of $\mathrm{Nb}$-doped $\mathrm{BaTiO}_{3}$ up to $x=0.20$. The possible explanation provided by Kolodiazhnyi \& Wimbush (2006) was that the Ti ions acquired extra electron from $\mathrm{Nb}$-doping, becoming trivalent (which was confirmed by our ELNES result), and that two adjacent trivalent Ti ions can be bounded together forming a spin-singlet small bipolaron $(S=0)$ at low 

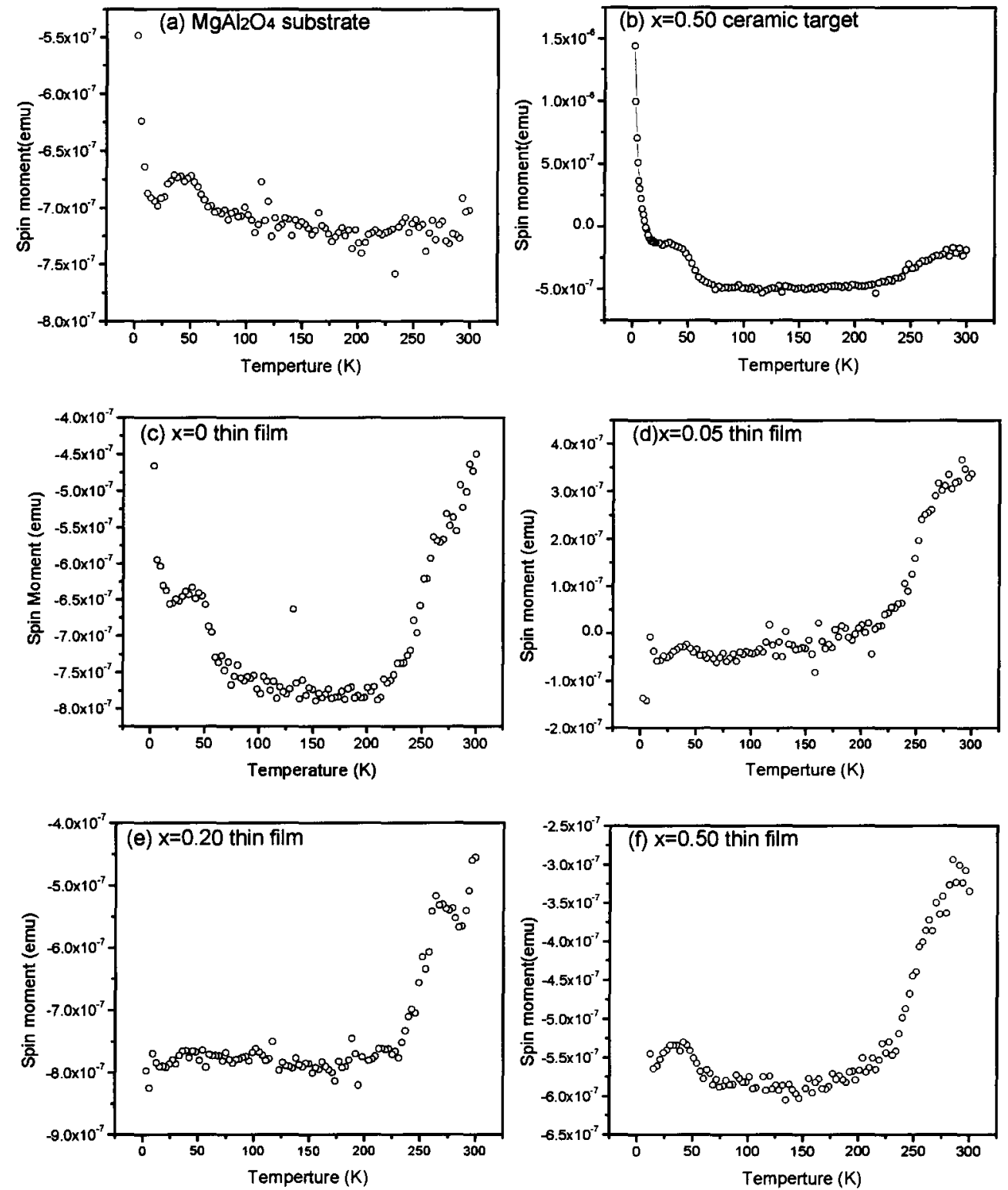

Figure 7.24: Temperature dependent spin moments of (a) the $\mathrm{MgAl}_{2} \mathrm{O}_{4}$, (b) $\mathrm{x}=0.50 \mathrm{Nb}$ doped ceramic target, (c) $x=0$ thin film, (d) $x=0.05 \mathrm{Nb}$-doped $\mathrm{BaTiO}_{3}$ thin film, (e) $\mathrm{x}=0.20$ $\mathrm{Nb}$-doped $\mathrm{BaTiO}_{3}$ thin film, and (f) $\mathrm{x}=0.50 \mathrm{Nb}$-doped $\mathrm{BaTiO}_{3}$ thin film.

temperature and dissolved into two separated small polarons $(S=1 / 2$ for each small polaron) as temperature increase, resulting in an increase of total spin moment at 
high temperature range. According to the prediction of the small singlet bipolaron, a maximum in the susceptibility will appear with increasing temperature and at even higher temperature the spin moment will decrease again. However due to the temperature limitation of our PPMS instrument, we were not able to heat the samples at temperature higher than $300 \mathrm{~K}$, and we were not able to locate a maximum on our curves.

A more recent report (Kolodiazhnyi, 2008) on the n-type $\mathrm{BaTiO}_{3-\delta}$ suggested that such abnormal magnetic susceptibility might be from the lifting of the electron degeneracy of the conduction band in the low-symmetry phases of the $\mathrm{BaTiO}_{3}$ at low temperature. The conduction band in low-symmetry phase might split into lower and upper bands separated by a small energy gap, 3-7 meV (i.e., 30-75K), and the low temperatures magnetic susceptibility was dominated by the lower band which was almost completely filled, resulting in a rather small magnetic susceptibility response at low temperature. However, in the $\mathrm{BaTi}_{1-x} \mathrm{Nb}_{x} \mathrm{O}_{3}$ system, a cubic phase of polycrystalline $\mathrm{BaTi}_{1-x} \mathrm{Nb}_{x} \mathrm{O}_{3}(0.1 \leq x \leq 0.2)$ remained down to at least $20 \mathrm{~K}$ by low-temperature X-ray analysis (Kolodiazhnyi \& Wimbush, 2006), which doesn't support the existing of a low-symmetry phase. A detailed neutron diffraction would be required to explore the low temperature structure of $\mathrm{BaTi}_{1-x} \mathrm{Nb}_{x} \mathrm{O}_{3}$, and band structure picture from the $1^{\text {st }}$ principle calculation would also need conduct to clarify the above hypothesis of conduction band split.

Besides the abnormal increase of spin moment at higher temperatures for the $\mathrm{Nb}$-doped $\mathrm{BaTiO}_{3}$ samples, a sharp decrease of spin moment of the $\mathrm{Nb}$-doped $\mathrm{BaTiO}_{3}$ thin film samples was also found. This effect could result from presence 
of a superconducting phase. However, the spin moments measurements represent the combined signals from both substrate and films. In order to subtract the spin moment contribution of the substrate, we firstly separated the total magnetic susceptibility into two parts: one is from the thin film and the other is from the substrate (as expressed as equation 7.2).

$$
\chi_{t}=\chi_{f}+\chi_{s}
$$

The subscript $t, f, s$ represented total, film and substrate, respectively. Secondly, we divided the total mass of the film from both sides of the equation 7.2 , and replaced the $\chi_{f}$ and $\chi_{s}$ by the magnetic susceptibility per gram $\left(\chi_{g, f}\right.$ and $\left.\chi_{g, s}\right)$ multiplied by the mass $\left(g_{f}\right.$ and $\left.g_{s}\right)$.

$$
\frac{\chi_{t}}{g_{t}}=\frac{\chi_{g, f} \cdot g_{f}}{g_{t}}+\frac{\chi_{g, s} \cdot g_{s}}{g_{t}}
$$

According to the definition of magnetic susceptibility, we change the total magnetic susceptibility by the total spin moment $\left(M_{t}\right)$ divided by the magnetic field $(H)$. We also change the mass by the density $(\rho)$ multiplying with the film surface area $(s)$ and the height $(h)$. Then we translate equation 7.3 further to equation 7.4 .

$$
\frac{M_{t} / H}{g_{t}}=\frac{\chi_{g, f} \cdot \rho_{f} \cdot s_{f} \cdot h_{f}}{\rho_{f} \cdot s_{f} \cdot h_{f}+\rho_{s} \cdot s_{s} \cdot h_{s}}+\frac{\chi_{g, s} \cdot \rho_{s} \cdot s_{s} \cdot h_{s}}{\rho_{f} \cdot s_{f} \cdot h_{f}+\rho_{s} \cdot s_{s} \cdot h_{s}}
$$

Since the surface area is the same for the substrate and the thin film $\left(s_{f}=s_{s}\right)$, we can cancel them out. Finally we obtain equation 7.5.

$$
\frac{M_{t} / H}{g_{t}}=\frac{\chi_{g, f}}{1+\frac{\rho_{s} \cdot h_{s}}{\rho_{f} \cdot h_{f}}}+\frac{\chi_{g, s}}{\frac{\rho_{f} \cdot h_{f}}{\rho_{s} \cdot h_{s}}+1}
$$


In equation 7.5 , the total spin moment $\left(M_{t}\right)$ is an experimental result; the magnetic field $(H)$ is the experimental parameter we set; the total weigh $\left(g_{t}\right)$ of the sample can be measured; the thickness of the substrate is measured by vernier caliper, and the thickness of the film can be measured from TEM cross section image; the magnetic susceptibility per gram of substrate can be obtained from the bare substrate measurement; the density of substrate can be obtained from XRD database; and the density of thin film can be calculated based on our XRD result assuming it's cubic. Finally the only remaining parameter-the magnetic susceptibility per gram of the thin film can be deduced and therefore the molar magnetic susceptibility of the thin film is obtained. Figure 7.25(b) shows the result of the magnetic susceptibility of the $x=0.50$ thin film after subtracting the substrate contribution. With the contribution of the film remained, the sharp decrease of magnetic susceptibility is more pronounced.

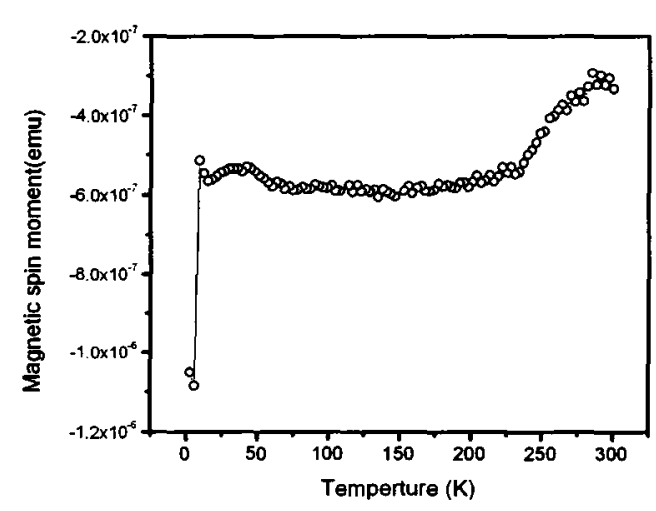

(a)

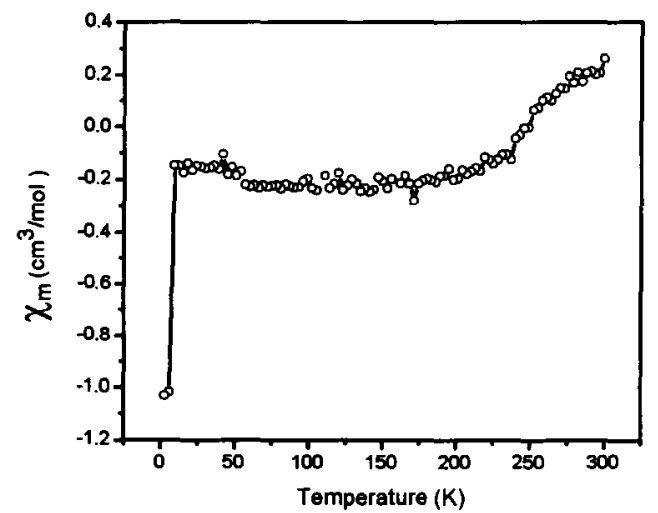

(b)

Figure 7.25: Magnetic properties of $x=0.50$ thin film. (a) The experimental magnetic spin moments (b) Mole magnetic susceptibility after subtracting the substrate's contribution

We repeated the experiment three times and the sharp decrease of susceptibility 
was always there. This suggests that a a superconducting phase forming in the film. This encouraged us to repeat the experiment around the critical temperate $(2 \mathrm{~K}-10 \mathrm{~K})$ with a fine temperature step $(1 \mathrm{~K})$ under different $\mathrm{AC}$ magnetic fields $(5 \mathrm{Oe}, 20 \mathrm{Oe}$, $100 \mathrm{Oe}, 200 \mathrm{Oe}$, and $500 \mathrm{Oe}$ ) with a 4 Oe DC magnetic field, shown in figure 7.26. The possible superconducting phase transition occured below 20 Oe and the critical temperature is between $6-8 \mathrm{~K}$, but it was completely washed out by the magnetic field higher than 100 Oe.
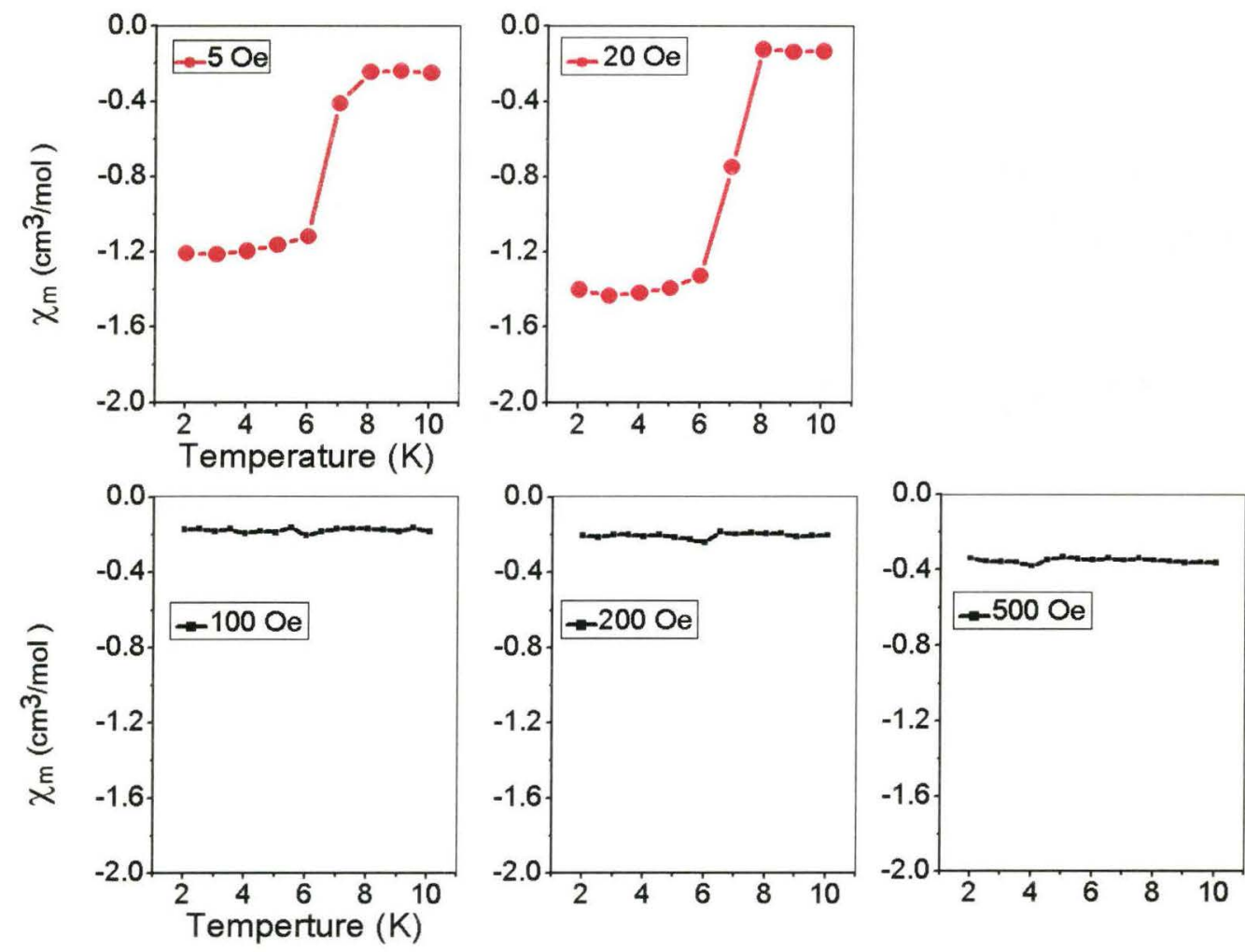

Figure 7.26: Molar magnetic susceptibilities of $x=0.50 \mathrm{Nb}$-doped $\mathrm{BaTiO}_{3}$ thin film under different magnetic fields

Since $\mathrm{BaNbO}_{3-\delta}(0.6<\delta<1)$ is reported to be a superconductor with a $\mathrm{T}_{c}$ of 14-22 K (Strukova et al., 1997; Gasparov et al., 2001), and very small amount Nb- 
doped $\mathrm{SrTiO}_{3}$ is also a superconductor with a $\mathrm{T}_{c}$ of $0.370 \mathrm{~K}$ (Schooley et al., 1964; Pfeiffer \& Schooley, 1969; Koonce et al., 1967). These make our $x=0.50 \mathrm{Nb}$-doped $\mathrm{BaTiO}_{3}$ thin film a possible superconductor that has confirmed by magnetic susceptibility measurement but still need to be confirmed by resistivity measurement.

However, our resistivity measurement (figure 7.27) does not support the existence of a superconducting phase. The $x=0.50 \mathrm{Nb}$-doped $\mathrm{BaTiO}_{3}$ thin film used for resistivity measurement with four gold contacts and a cap layer on top showed a metallic resistivity behavior with an abnormal resistivity decrease when the temperature was lower than $20 \mathrm{~K}$ but it did not fall to zero value.

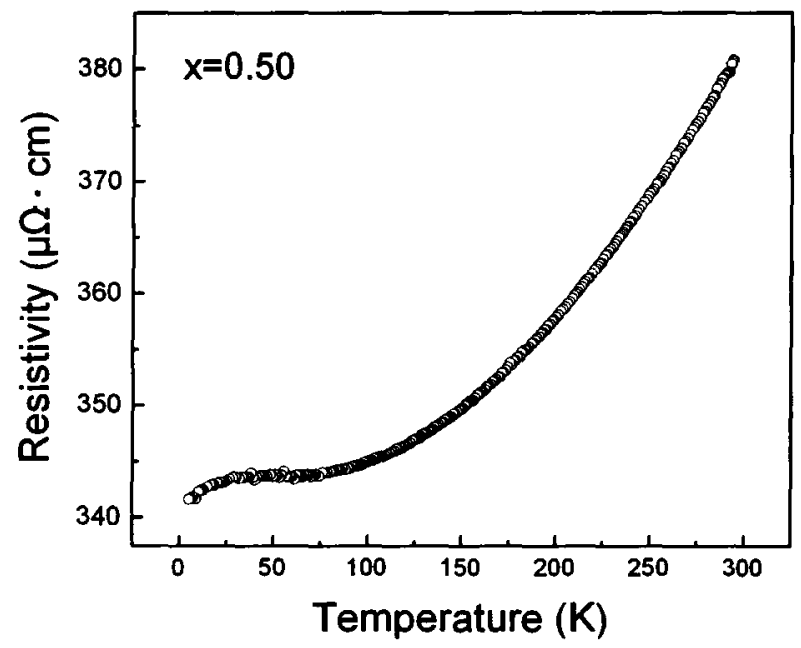

Figure 7.27: The resisitivity dependence of temperature for $x=0.50 \mathrm{film}$. There was an abnormal resistivity decrease when the temperature was lower than $20 \mathrm{~K}$ but it did not fall to zero value 


\subsection{Electron density of states calculation}

In order to understand how the electronic structure of the $\mathrm{BaTi}_{1-x} \mathrm{Nb}_{x} \mathrm{O}_{3}$ samples changes with $\mathrm{Nb}$ doping, we calculated the electron density of states (DOS) for $x=0.125,0.25,0.50,0.75$, and 1.0 samples based on $2 \times 2 \times 2$ ordered cubic structures shown in figure 7.28 where $\mathrm{Nb}$ atoms homogenously distribute in the lattice. From the diffraction patterns of $x=0.50$ sample (see section 7.6 on page 105), we have demonstrated that there is no ordered phase, which intrinsically implies that the modeled structure (used in the calculations) is not accurate. Calculations with ordered structure, however, are still a good starting point since disordered lattice calculations would be practically impossible. A widely used program for electronic structure calculation under the framework of the density functional theory (DFT)WIEN2k, is dependent on a periodic structure and cannot be used for a random substitutional case. However, even if the assumed ordered structures are not fully realistic, we still can qualitatively understand the effect of $\mathrm{Nb}$ doping using the WIEN2k program.

The calculated total densities of states with the assumed structures are shown in figure 7.29. The shapes of the valence bands are almost the same. The positions systematically shift to lower energy and the Fermi level moves within the conduction band. The reason could be that the relatively simple electron configurations of $\mathrm{Nb}$ atoms $\left(\mathrm{Kr} 4 \mathrm{~d}^{3} 5 \mathrm{~s}^{2}\right)$ and highly ionized nature, cause the nature of the bonds between $\mathrm{Nb}$ ions and adjacent oxygen ions are more ionized. Therefore $\mathrm{Nb}$ ions only weakly hybridized with adjacent oxygen ions and not significantly modify the 


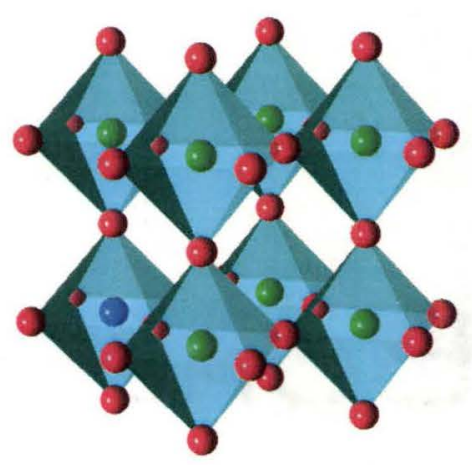

(a) $\mathrm{x}=0.125$

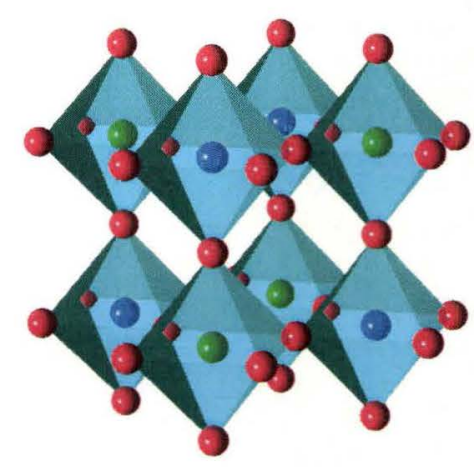

(c) $\mathrm{x}=0.50$

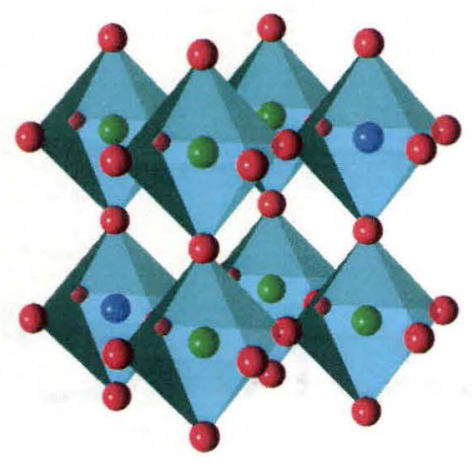

(b) $x=0.25$

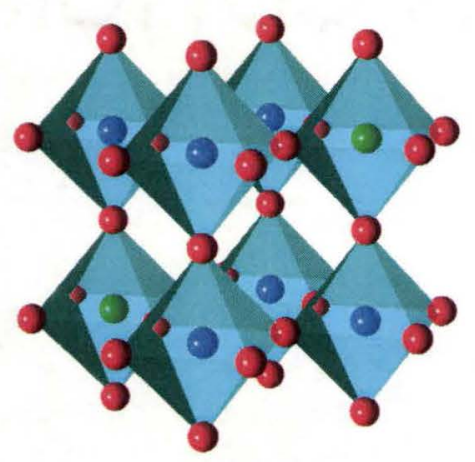

(d) $\mathrm{x}=0.75$

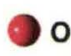

$\mathrm{Nb}$

$\mathrm{Ti}$

Figure 7.28: $2 \times 2 \times 2$ ordered structure at $(a) x=0.125,(b) x=0.25,(c) x=0.50$, and $(d) x=0.75$ composition for calculation use. Here the $\mathrm{Ba}$ ions are not shown to improve clarity

valence band. The Fermi level lies in the conduction band for all compositions with the ideal and thus indicate that the material is metallic in ground state. However, this prediction disagrees with our experimental results where we demonstrated that only the highly $\mathrm{Nb}$ doped $(\mathrm{x} \geq 0.50)$ thin films show metallic behavior at low temperature (see section 7.8 on page 121). This conflict therefore suggests that Anderson metal insulator transition may exist due to the $\mathrm{Nb}$ random distribution (see section 7.6 on page 105$)$. 


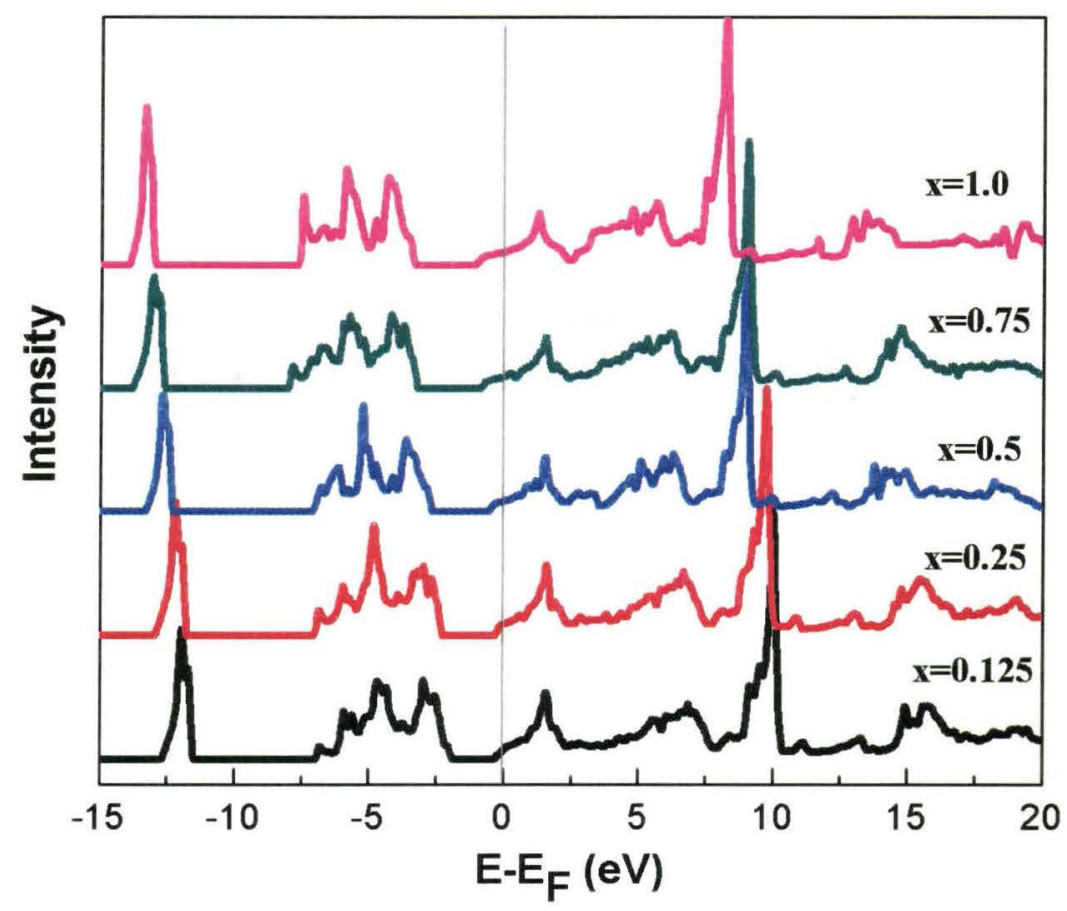

Figure 7.29: The calculated electron density of state (DOS) for $x=0.125,0.25,0.50$, 0.75 , and 1.0 samples based on $2 \times 2 \times 2$ ordered cubic structures (figure 7.28 ) by WIEN2k program. The valence states for all compositions are almost the same, and the Fermi level cross the conduction band for all compositions indicates they are supposed to be metallic at ground state

\subsection{Electron density calculation}

From the previous DOS calculation, we have shown that the conduction bands extend below the Fermi level. But, how these conduction electrons distribute themselves in the real space. Do do these conduction elections form uniform electron gas (like a metal) or localize themselves on some specific atom sites? Here we still focus on the $x=0.50$ conducting film and use the electron density calculation to plot the charge density distribution of conduction elections. 
Figure 7.30 shows a detail of the total DOS of $x=0.50$ conducting film around the Fermi level. The area in blue color represents the density of conduction electrons. Projecting this density onto the crystal (001) plane, we obtain a distribution of the corresponding 2D conduction electron density (figure 7.31). The PDOS of $\mathrm{Ti}, \mathrm{Nb}$ and $\mathrm{O}$ ions are shown in figure 7.32. Fermi level in figure 7.32 are set to zero and crosses the Ti $3 d-t_{2 g}$ band, $\mathrm{Nb} 4 d-t_{2 g}$ band and $\mathrm{O} 2 p$ band. There are more conduction electrons on $\mathrm{Ti}$ sites than that on $\mathrm{Nb}$ sites. The conduction electrons on both $\mathrm{Ti}$ sites and $\mathrm{Nb}$ sites are belong to the $d$ band $\mathrm{t}_{2 g}$ orbitals. Therefore the symmetric pattern of the conduction electron density around $\mathrm{Ti}$ and $\mathrm{Nb}$ sites in figure 7.31 is the characteristic of $d$ band electrons with $\mathrm{t}_{2 g}$ symmetry. The conduction electron distribution in figure 7.31 is not a uniform electron gas but located around the $\mathrm{Ti}$ and $\mathrm{Nb}$ ions. The stoichiometric $\mathrm{BaTiO}_{3}$ is an insulator with tetravalent $\mathrm{Ti}$ ions and there should be no conduction electrons localized on the Ti sites. The conduction electron density found on $\mathrm{Ti}$ sites of $\mathrm{BaTi}_{0.5} \mathrm{Nb}_{0.5} \mathrm{O}_{3}$ film indicates that the oxidation state of Ti ion is reduced and not tetravalent any more, which on the other side confirms the Ti valence change revealed by EELS (see section 7.13 on page 112). As a balance of the reduced $\mathrm{Ti}$ oxidation state, the $\mathrm{Nb}$ ions are approaching to pentavalency.

A conduction electron density projection in figure 7.31 also reveals that there are much more conduction electrons on $\mathrm{Ti}$ sites than that on $\mathrm{Nb}$ sites, and the conduction electrons of $\mathrm{O} 2 p$ band slightly deflect towards adjacent Ti sites rather than $\mathrm{Nb}$ sites. Hence the Ti site localized more conduction electrons in its $3 d-t_{2 g}$ band and such conduction elections could hybridize with $\mathrm{O} 2 p$ band. This give a pos- 


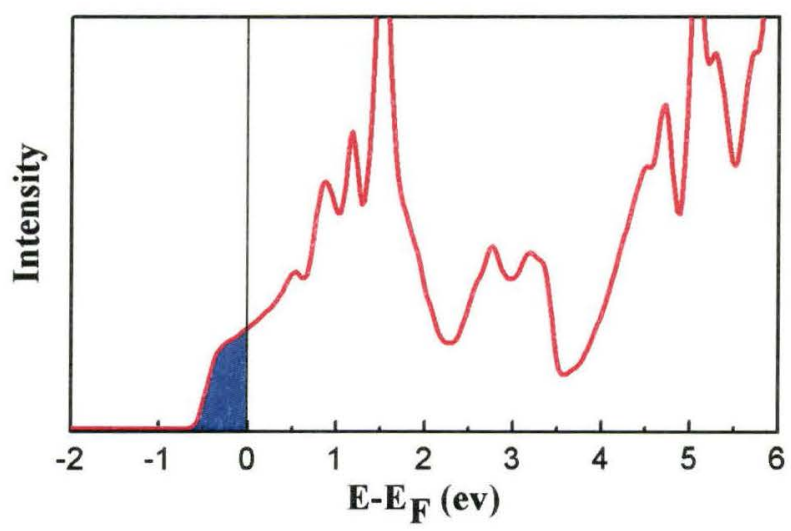

Figure 7.30: DOS of $x=0.50$ conducting film around Fermi level

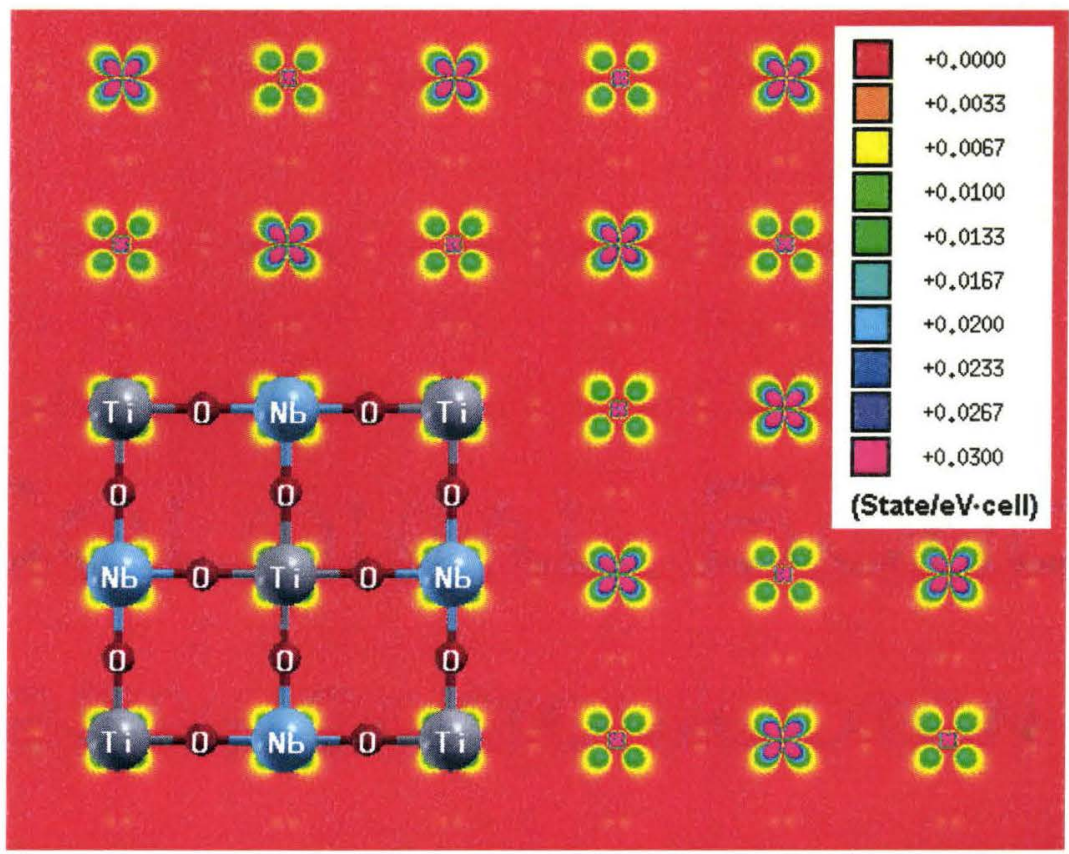

Figure 7.31: Conduction electron density projection onto (001) plane for $x=0.5$ film

sible clue that the hybridization between $\mathrm{Ti}$ and $\mathrm{O}$ is much stronger than $\mathrm{Nb}$ and O. Although the conduction electrons might move along the $\mathrm{Nb}-\mathrm{O}-\mathrm{Ti}$ chains, most part of them would like to move between $\mathrm{Ti}$ and $\mathrm{O}$ sites. The electron transport 


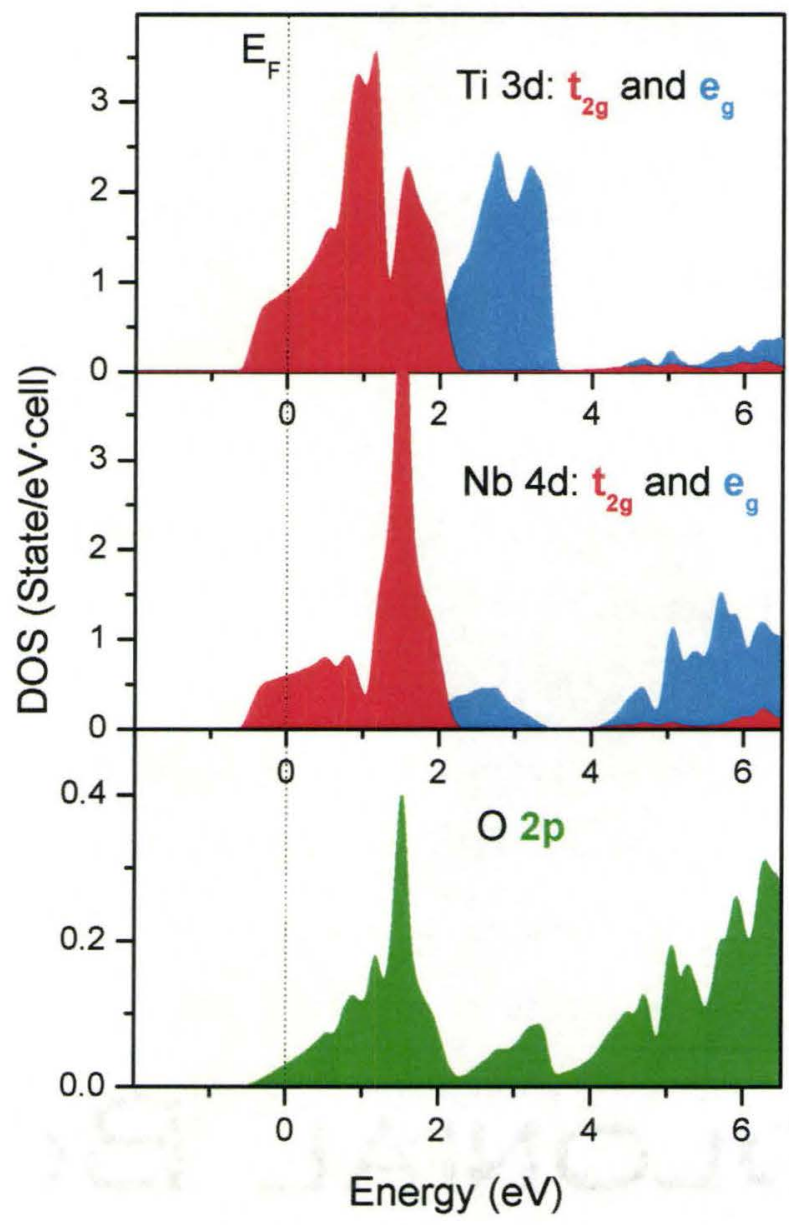

Figure 7.32: Site projected PDOS of $\mathrm{BaTi}_{0.5} \mathrm{Nb}_{0.5} \mathrm{O}_{3}$ with assumed ordered structure. Fermi level was set to zero and crossed the Ti $3 d-\mathrm{t}_{2 g}$ band, $\mathrm{Nb} 4 d-\mathrm{t}_{2 g}$ band and $\mathrm{O} 2 p$ band. There are more conduction electrons on $\mathrm{Ti}$ sites than that on $\mathrm{Nb}$ sites. The conduction electrons on both Ti sites and $\mathrm{Nb}$ sites were belong to the $d$ band $\mathrm{t}_{2 g}$ orbitals.

path therefore could mainly be formed by the Ti-O chains. If the electron jumping together with a structure distortion, a polaron conduction mechanism is favored. 


\subsection{Band structure and Fermi surface}

The energy band structure of $\mathrm{BaTi}_{0.5} \mathrm{Nb}_{0.5} \mathrm{O}_{3}$ was obtained by the linear augmented plane wave (LAPW) method. The assumed structure of $\mathrm{BaTi}_{0.5} \mathrm{Nb}_{0.5} \mathrm{O}_{3}$ (see figure 7.28(c) on page 139) forms an FCC Bravais lattice. The calculated band structure was shown in figure 7.33 at selected symmetry points in the first Brillouin zone. The high symmetry $\vec{k}$ points in the in the first Brillouin zone are illustrated in figure 7.34(a), where $\Gamma$ is at the center of the Brillouin zone, $X$ is located at the center of a square face, $K$ is in the middle of an edge joining two hexagonal faces, $L$ is at the center of a hexagonal face and $W$ is at the corner. The $\Delta$ represents the $<100>$ direction and $\Lambda$ represents the $<111\rangle$ direction. The three $t_{2 g}$ orbitals form the lower part of the conduction band and are degenerate at $\Gamma$. This degeneracy is split at other parts of the zone because of the different bonding interactions of orbitals with different orientations with respect to $\vec{k}$. The band energies at $\vec{k}=0$ of the three $t_{2 g}$ orbitals are of non-bonding transition metal $3 d$ orbitals. Two "branch" of the $t_{2 g}$ orbitals are upturn along the $\Gamma X$ direction (in real space is the $\left.<100\right\rangle$ direction indicates that a progressively stronger interaction between the transition metals $t_{2 g}$ orbitals and the oxygen $2 p$ orbitals. The increased $\vec{k}$ results in the formation of antibonding orbitals, with the energy trends shown. The corresponding bonding orbitals are in the valence band. This stronger interaction between transition metals and oxygens are due to the formation of $p d \pi$ between oxygen $p_{x}$ and transition metals $d_{x y}$ and $d_{x z}$ orbitals. The other one "branch" of the three $t_{2 g}$ orbitals is nearly flat in the $\Gamma X$ direction indicates a rather weak interaction between the transition metal $t_{2 g}$ orbitals and the $\mathrm{O} 2 p$ orbitals. This is because the $d_{y z}$ orbitals are perpendicular to this direction and can not overlap with the oxygen orbitals. 
The Fermi surface is defined as the surface of a constant energy in $\vec{k}$ space to separate the occupied states from the unoccupied states. It plays an important role of a metallic or semiconducting solid in determining the transport properties of the material. Figure 7.34 shows the calculated Fermi surface of the cubic $\mathrm{BaTi}_{0.5} \mathrm{Nb}_{0.5} \mathrm{O}_{3}$ sample with the help of XcrysDen program (Kokalj, 2003). The three separated 'sheets' arise from different parts of the $t_{2 g}$ conduction band, which across the Fermi level at different $\vec{k}$ points. The three sheets of the $\mathrm{BaTi}_{0.5} \mathrm{Nb}_{0.5} \mathrm{O}_{3}$ Fermi surface are labeled as $\alpha, \beta$, and $\gamma$, respectively. Each sheet corresponds to an electron-like surface centered at $\Gamma$. The $\alpha$ and $\beta$ sheets were closed, and the $\gamma$ sheet was open along $<100>$. The open sheet consisted of three intersecting cylinders. This calculated three-sheet Fermi surface structure was the same as the metallic oxide $\mathrm{ReO}_{3}$ (Mattheiss, 1969) and the cubic $3 \mathrm{~d}^{1}$ perovskite $\mathrm{CaVO}_{3}$ (Inoue et al., 2002).

The Fermi surface calculation result supports an assumption of 2D conduction mechanism, because the Fermi surface sheets of our system are basically formed from three branches of the $t_{2 g}$ band containing the $d_{x y}, d_{x z}$, and $d_{y z}$ states, and each has a two dimensional character.

In summary, $\mathrm{BaTi}_{1-x} \mathrm{Nb}_{x} \mathrm{O}_{3}$ films, spanning the entire range of $x$, have been deposited on $\mathrm{MgAl}_{2} \mathrm{O}_{4}$ substrates. X-ray diffraction measurements indicate single phase films for all values of $x$, contrary to a previously reported niobium solubility limit. Films show extreme sensitivity to high temperature oxygen exposure, which destroys conductivity and severely disrupts crystallinity. The 2D XRD shows there are nine possible grain orientations and possible twin structures. Linear $I-V$ curve 


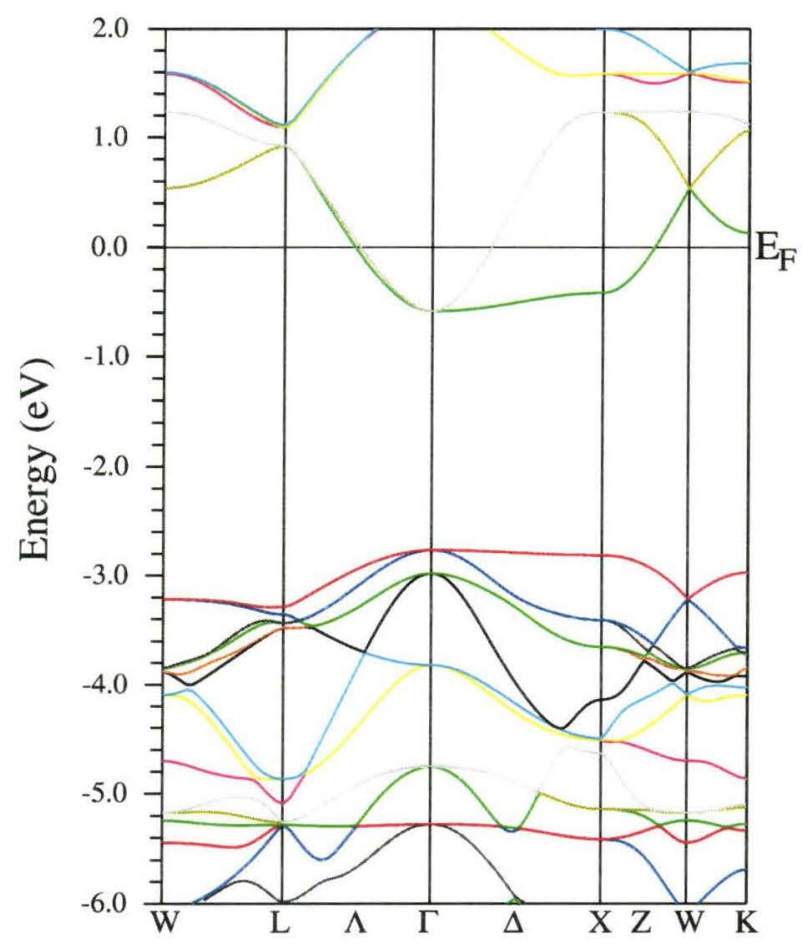

Figure 7.33: Energy band structure of $\mathrm{BaTi}_{0.5} \mathrm{Nb}_{0.5} \mathrm{O}_{3}$ obtained by WIEN2k package at symmetry points. The conduction bands are formed by the $3 t_{2 g}$ orbitals

results confirm the presence of Ohmic contacts. Hall coefficient measurements indicate a change of major charge carriers from holes to electrons. Under optimum growth conditions increasing $x$ gives rise to $\mathrm{Ti}^{4+}$ to $\mathrm{Ti}^{3+}$ transformation in the oxidation state accompanied by increased conductivity with a semiconductor-metal transition near $x=0.2$. Temperature dependent magnetic measurements show an anomalous rise in the spin moment, a feature supportive of the small singlet bipolaron model. The DOS calculations show Fermi level move in the conduction band as increase $x$, and electron density projection shows that such conduction elections are likely to be localized on the $\mathrm{Ti}$ sites, i.e., they possess a strong Ti character. 


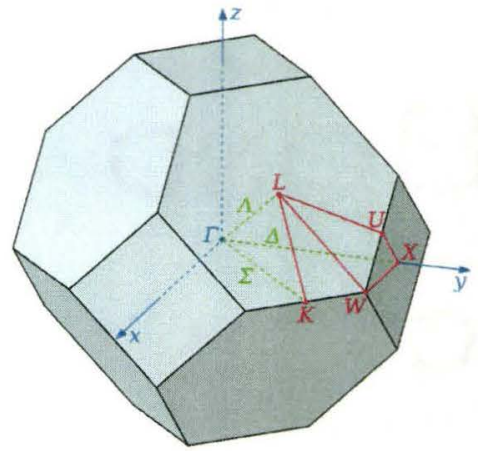

(a)

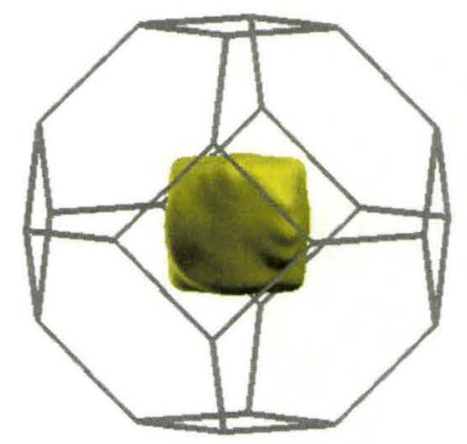

(c) $\beta$

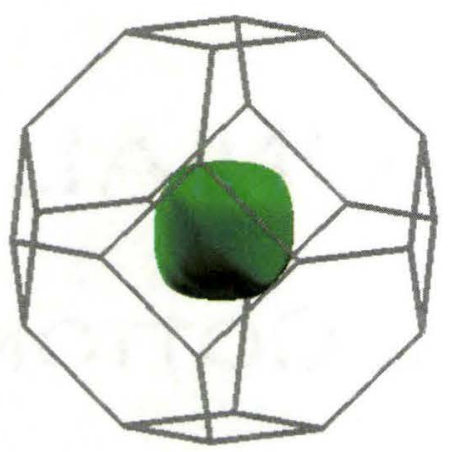

(b) $\alpha$

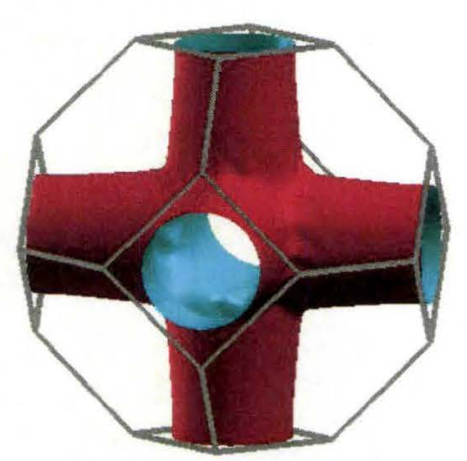

(d) $\gamma$

Figure 7.34: Fermi surface calculation of the $x=0.5$ sample with assumed structure. (a) The first Brillouin zone for the face-centered cubic lattice. (b) The first type of Fermi surface labeled as $\alpha$. (c) The second type of Fermi surface labeled as $\beta$. (d) The third type of Fermi surface labeled as $\gamma$. 


\section{Chapter 8}

\section{$\operatorname{BaTi}_{0.5} \mathbf{N b}_{0.5} \mathrm{O}_{3}$ Thin Films on (001), (011) and (111) $\mathrm{MgAl}_{2} \mathrm{O}_{4}$ Substrate}

The $\mathrm{BaTi}_{0.5} \mathrm{Nb}_{0.5} \mathrm{O}_{3}$ thin film on (001) $\mathrm{MgAl}_{2} \mathrm{O}_{4}$ substrate demonstrates good conductivity and lower lattice mismatch with potential PZT/PT ferroelectric materials. In order to study the film's properties in different orientations, (001), (011) and (111) orientated $\mathrm{MgAl}_{2} \mathrm{O}_{4}$ substrates are used to explore the films quality as well as resistivity. 


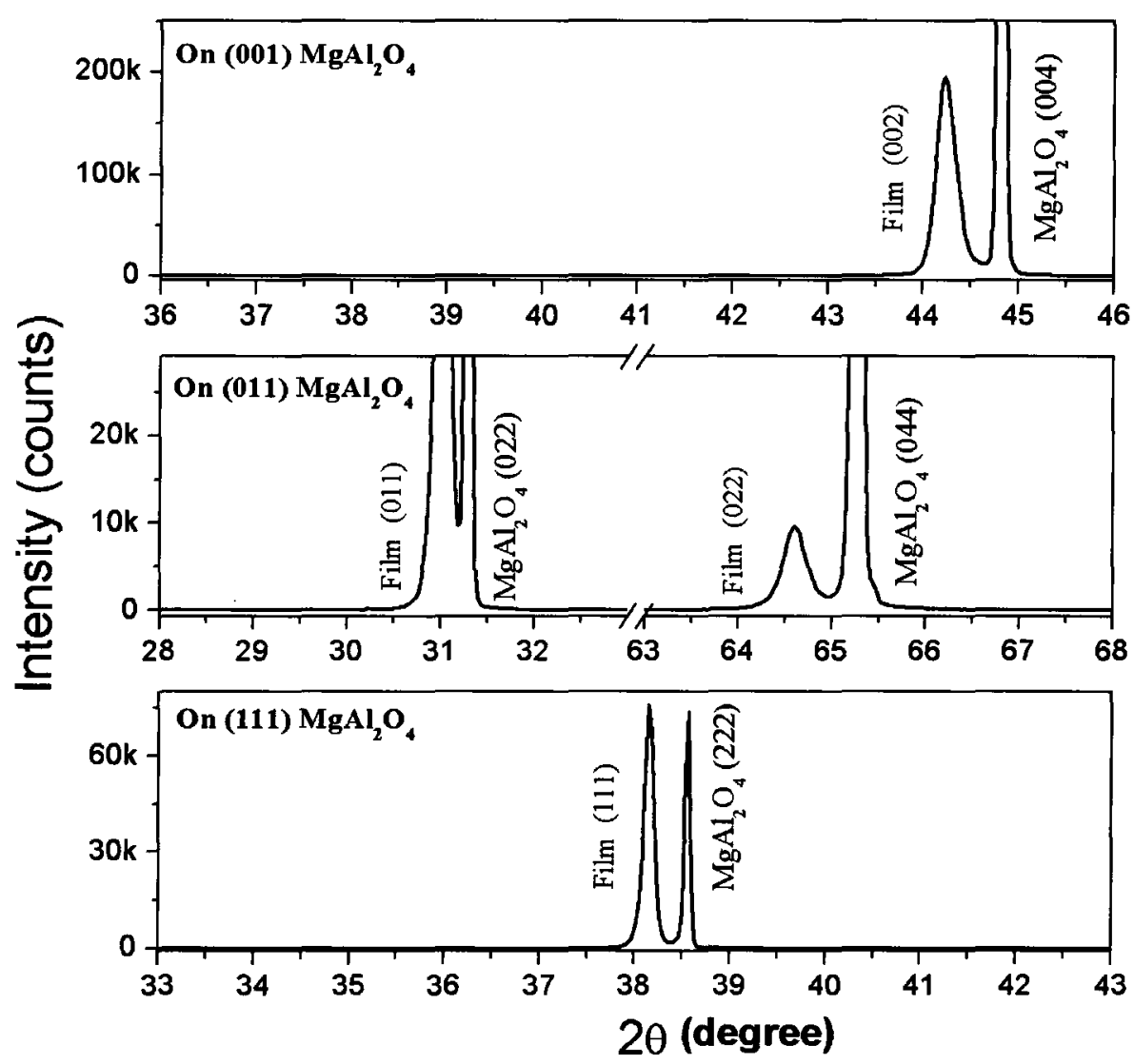

Figure 8.1: XRD results of $\mathrm{BaTi}_{0.5} \mathrm{Nb}_{0.5} \mathrm{O}_{3}$ thin films on (001), (011) and (111) orientated $\mathrm{MgAl}_{2} \mathrm{O}_{4}$ substrate depositing at $850^{\circ} \mathrm{C}$.

\subsection{XRD results}

$\mathrm{BaTi}_{0.5} \mathrm{Nb}_{0.5} \mathrm{O}_{3}$ thin films were deposited at $850{ }^{\circ} \mathrm{C}$ on $(001) \mathrm{m}(011)$ and $(111)$ orientated $\mathrm{MgAl}_{2} \mathrm{O}_{4}$ substrate using PLD. The XRD spectra are shown in figure 8.1. All three different orientated films are phase pure, and only (00h), (0hh) and (hhh) reflections of film and substrate appear on each XRD plot. There are no other peaks found and the films are highly orientated. Assuming a cubic structure, the lattice parameter can be achieved from each XRD measurement with a little 
Table 8.1: The value of lattice parameter $c$ of different orientated $\mathrm{BaTi}_{0.5} \mathrm{Nb}_{0.5} \mathrm{O}_{3}$ films on $\mathrm{MgAl}_{2} \mathrm{O}_{4}$ substrate deposition at $850^{\circ} \mathrm{C}$ by assuming a cubic structure.

\begin{tabular}{ccc}
\hline $\mathrm{BaTi}_{0.5} \mathrm{Nb}_{0.5} \mathrm{O}_{3}$ & Lattice parameter $c(\AA)$ & lattice mismater \\
\hline$(001)$ orientated film & $4.092(2)$ & $1.25 \%$ \\
$(011)$ orientated film & $4.076(2)$ & $0.854 \%$ \\
$(111)$ orientated film & $4.083(2)$ & $1.03 \%$ \\
\hline
\end{tabular}

difference as listed in table 8.1.

For the $(001)$ orientated $\mathrm{BaTi}_{0.5} \mathrm{Nb}_{0.5} \mathrm{O}_{3}$ film, increasing the deposition temperate from 790 to $850{ }^{\circ} \mathrm{C}$ slightly reduced the lattice parameter from $4.098 \AA$ (see table 7.1 on page 95 ) to $4.092 \AA$, and also improved the film quality in terms of single orientation. Compared to the $(001)$ orientated $\mathrm{BaTi}_{0.5} \mathrm{Nb}_{0.5} \mathrm{O}_{3}$ film, the lattice parameter of the (011) and (111) orientated films decreased, giving a smaller lattice mismatch to the $\mathrm{MgAl}_{2} \mathrm{O}_{4}$ substrate. The reason that different lattice parameter we obtained here could be due to the distortion of the lattice which would result in a non-perfect cubic structure, or due to the different surface atom distribution of the substrate at different orientation which would result in different strains onto the film.

\subsection{Thin film surface morphology}

The surface morphology of conducting $\mathrm{BaTi}_{0.5} \mathrm{Nb}_{0.5} \mathrm{O}_{3}$ films at different orientations is shown in figure 8.2 at different magnifications. For the (001) orientated film (figure 8.2(a)), a rough surface with small exposed grain size of $50-100 \mathrm{~nm}$ 

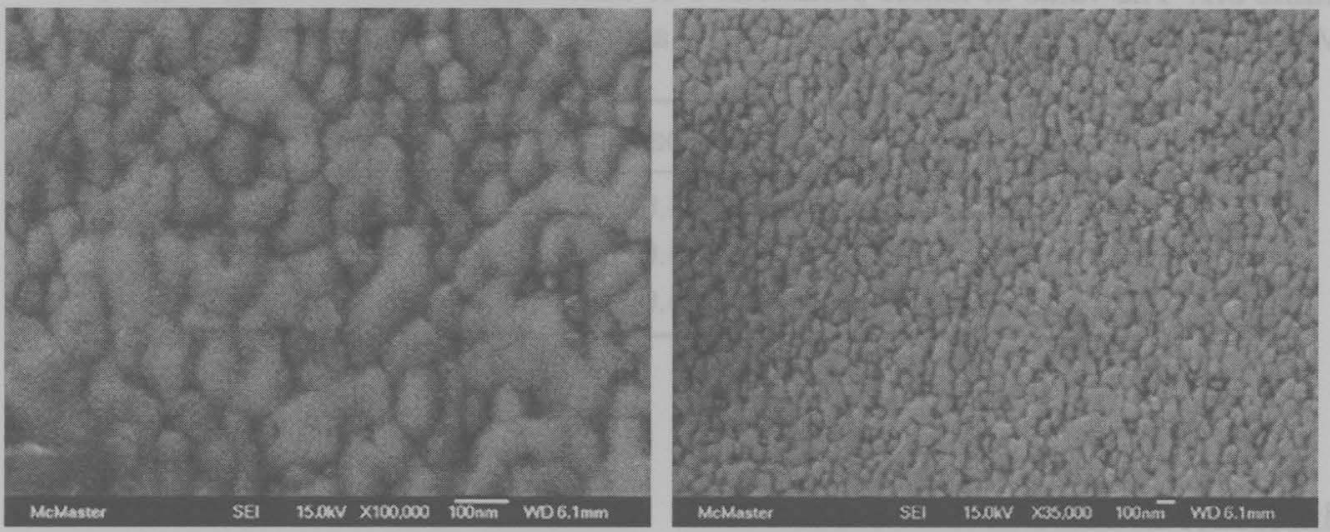

(a) (001) orientated film
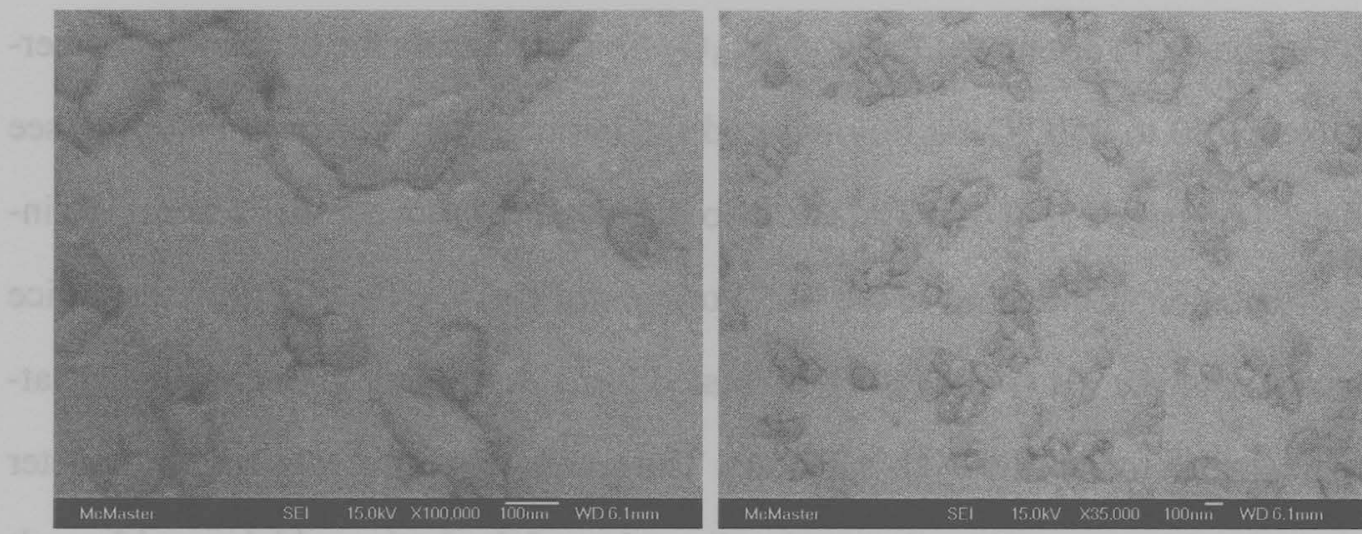

(b) (011) orientated film
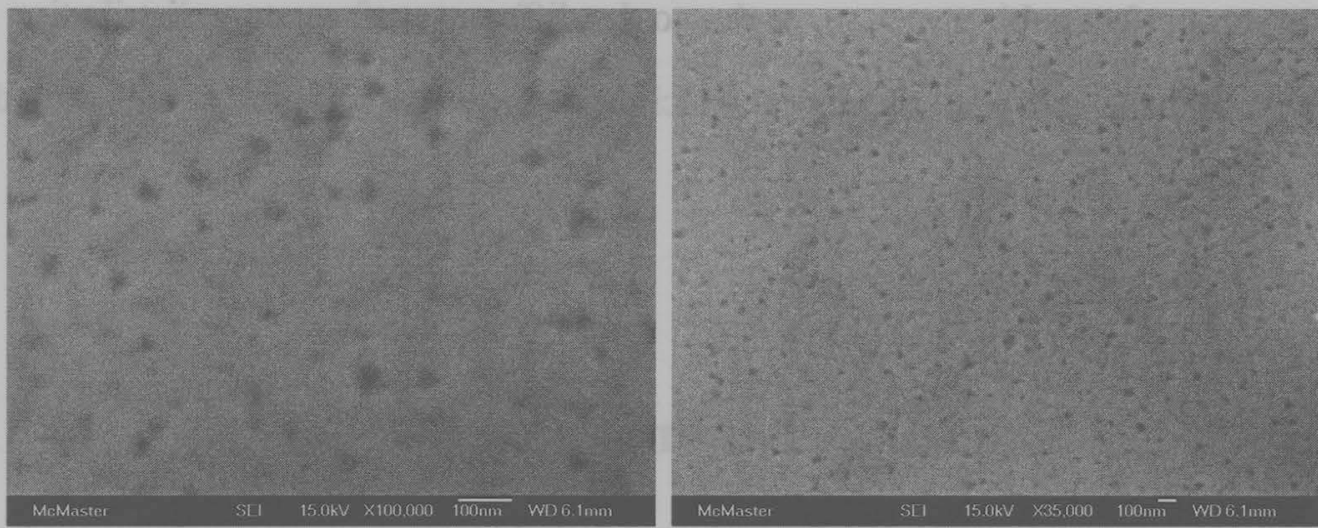

(c) (111) orientated film

Figure 8.2: SEM images of $\mathrm{BaTi}_{0.5} \mathrm{Nb}_{0.5} \mathrm{O}_{3}$ thin films on (a) (001) orientated and (b) (011) orientated and (c) (111) orientated $\mathrm{MgAl}_{2} \mathrm{O}_{4}$ substrate at $850{ }^{\circ} \mathrm{C}$. 
can be found, however, the grain size is not uniform. The small grains are round with a size of $50 \mathrm{~nm}$, while large grain is elliptic elongated with a $50 \mathrm{~nm}$ width and 100-200 $\mathrm{nm}$ length. The large grain is the result of two or more small grains coalescence. Besides clearly resolved grains, there are still some tiny particles randomly dispersed at grain boundary. They could be the undissolved droplets on surface, remaining grains after Ostwarld Ripening process or simply tiny grains with other orientations. The film appears dense and less porous. Comparing the (011) and (111) orientated films (figure 8.2(b) and 8.2(c)) to the (001) orientated $\mathrm{BaTi}_{0.5} \mathrm{Nb}_{0.5} \mathrm{O}_{3}$ film (figure 8.2(a)), it is clear that the grains appear much larger although there are some small scattered grains or intergrain boundaries. For (111) orientated films, although grain boundaries still can be seen, there is no scattered grains or particles and its surface appears very smooth. Both surface morphologies of (011) and (111) orientated films are much better than that of (001) orientated film, which could be the result of the smaller lattice mismatch.

\subsection{TEM analysis}

Figure 8.3 is a low magnification bright field (BF) image of the (001), (011) and (111) orientated $x=0.50$ films' cross-section obtained using a Philips CM 12 TEM. The thickness of the $(001)$ orientated film was about $270 \mathrm{~nm}$, and the thickness of the (011) and (111) orientated films was about $350 \mathrm{~nm}$. The film exhibited columnar bands with dark contrast, which extend from the substrate to the film's surface along a direction that is roughly perpendicular to the interface. Both three (001), (011) and (111) orientated films showed good epitaxy on the substrate. The width of the 


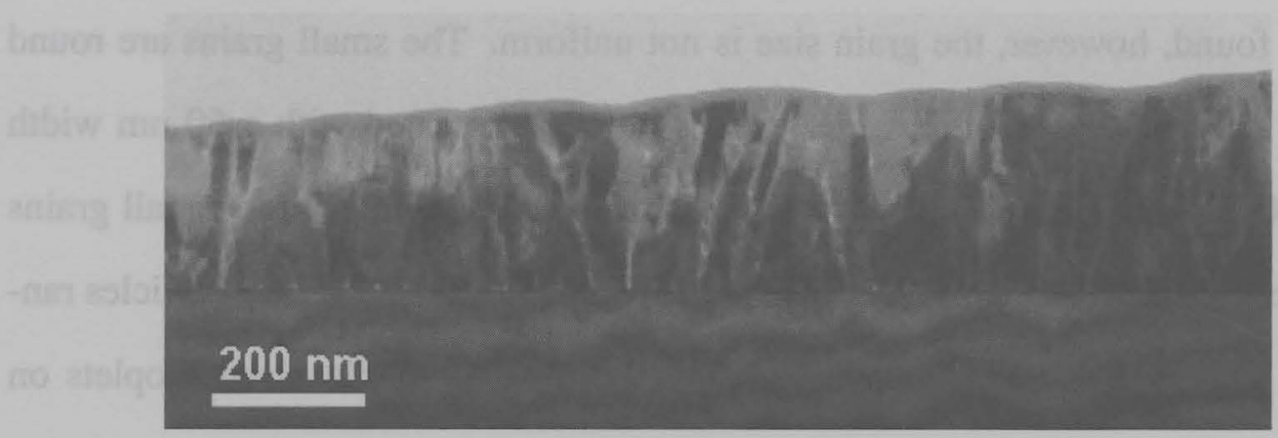

(a) (001) orientated film

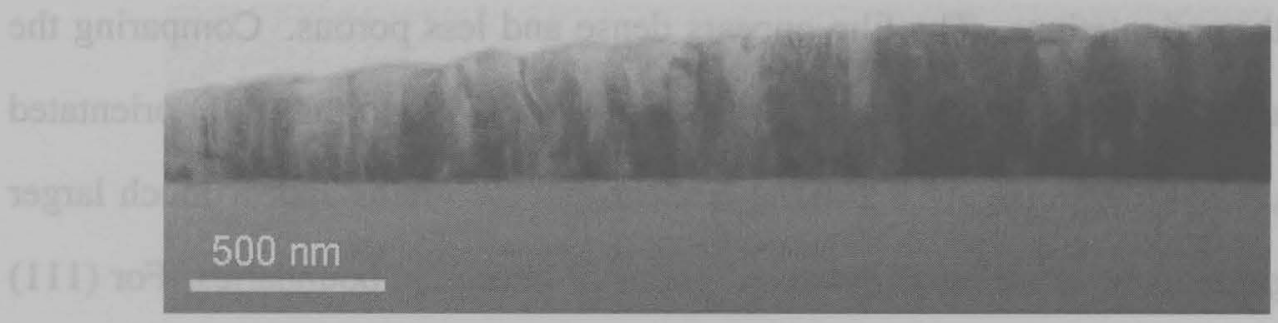

(b) (011) orientated film

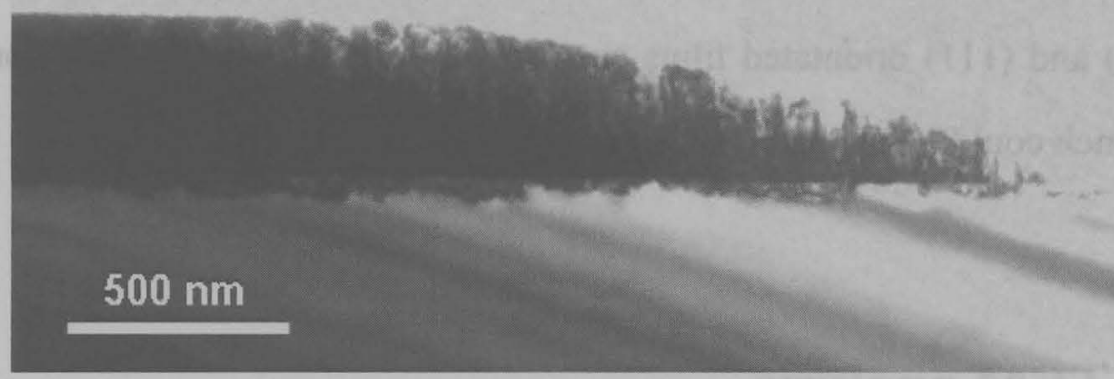

(c) (111) orientated film

Figure 8.3: Low magnification bright field TEM images of (a) (001) (b) (011) and (c) (111) orientated $\mathrm{BaTi}_{0.5} \mathrm{Nb}_{0.5} \mathrm{O}_{3}$ films showing a film-substrate combination in cross-section. The thickness variance along the films is the effect of the sample preparation. The real thickness of the film did not change along the films.

columnar structures was from $50-100 \mathrm{~nm}$. The thickness information will be used later to determine their resistivities. 

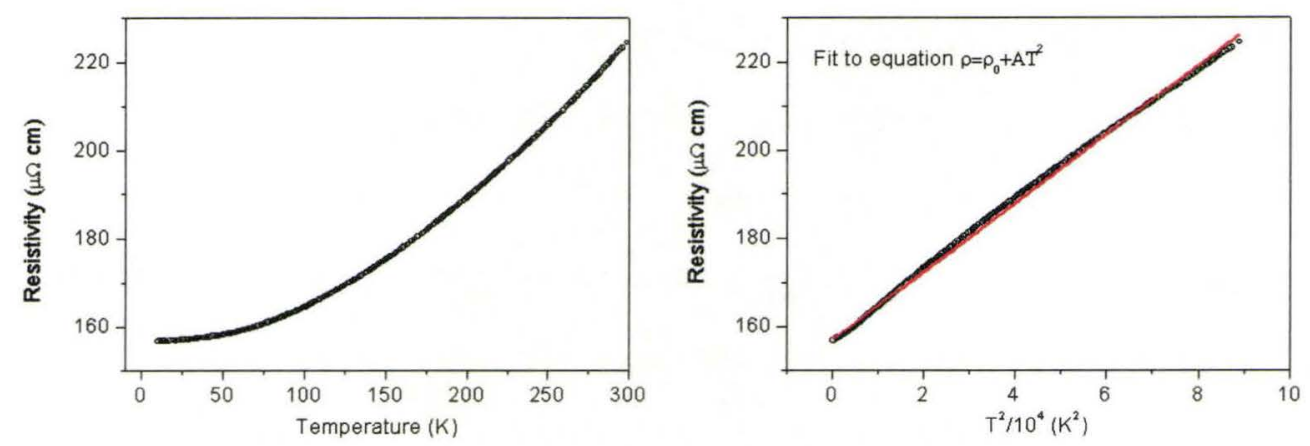

(a) (001) orientated film
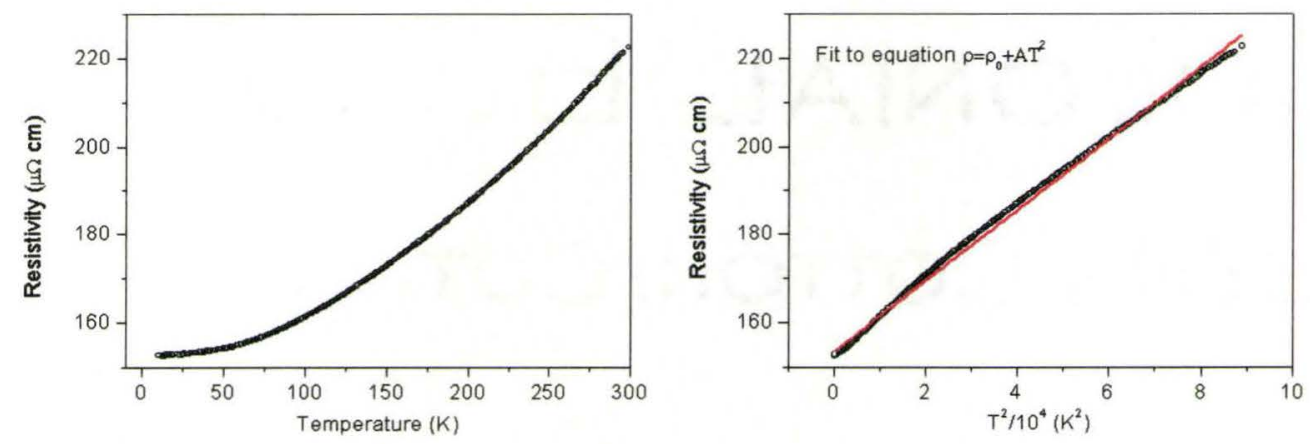

(b) (011) orientated film
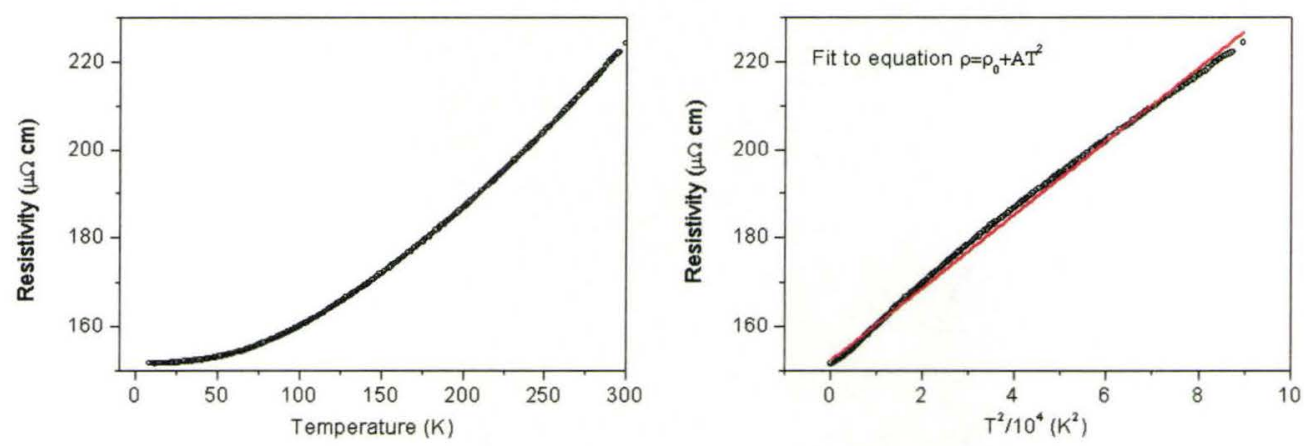

(c) (111) orientated film

Figure 8.4: Resistivity of (a) (001), (b) (011) and (c) (111) orientated $\mathrm{BaTi}_{0.5} \mathrm{Nb}_{0.5} \mathrm{O}_{3}$ films on $\mathrm{MgAl}_{2} \mathrm{O}_{4}$ substrate. The resistivity of each film can be fitted to the equation $\rho=$ $\rho_{0}+A T^{2}$. The open circle dots were experimental results and the red solid lines were fitted results

\subsection{Resistivity}


Figure 8.4 shows a comparison of the resistivity of (001), (011) and (111) orientated $\mathrm{BaTi}_{0.5} \mathrm{Nb}_{0.5} \mathrm{O}_{3}$ films. All films were metallic and had similar resistivity value over the testing temperature range. All three resistivity curves can be fitted to the equation $\rho=\rho_{0}+A T^{2}$ as shown in figure 8.4, indicating an electron-electron interaction dominating the resistivity behaviors of temperature dependence. 


\section{Chapter 9}

\section{$\mathrm{Ba}_{1-y} \mathrm{Sr}_{y} \mathrm{Ti}_{0.5} \mathrm{Nb}_{0.5} \mathrm{O}_{3}$ Thin Films on $\mathrm{MgAl}_{2} \mathrm{O}_{4}$ Substrate}

In chapter 7, we reported on the deposition of $\mathrm{BaTi}_{1-x} \mathrm{Nb}_{x} \mathrm{O}_{3}$ (BTNO) thin films over the entire range of $x$. It was demonstrated that with increased $\mathrm{Nb}$ content the material became increasingly conducting with a transition to metallic behavior near $x=0.2$, a value consistent with other reports (Marucco et al., 1997; Liu et al., 2005). Over this same range the lattice constants varied from 4.014 to $4.142 \AA$. Such values allow for an epitaxial relationship with the (001) $\mathrm{MgAl}_{2} \mathrm{O}_{4}(\mathrm{a}=8.083$ $\AA$ ) substrate used, where at the interface four unit cells of BTNO fit to a single unit cell of the substrate. Unfortunately, the values of $\mathrm{x}$ which match most closely to the substrate's unit cell are those not showing metallic behavior. These latticematched compositions were also the ones which showed the best crystallinity and surface morphology, with the metallic compositions displaying faceted surface fea- 
tures. Such a situation diminishes the effectiveness of BTNO as a bottom electrode material.

In this chapter, we describe our efforts to resolve this issue through a partial strontium-for-barium substitution. Such a substitution is well known to decrease the lattice parameter in the $\mathrm{Ba}_{1-y} \mathrm{Sr}_{y} \mathrm{TiO}_{3}$ system $[10,11]$. At the same time, it maintains the perovskite's A-site valence state, and hence, does not significantly alter the electronic transport properties $[12,13]$. Thus, it is expected that a similar substitution, for the $\mathrm{BaTi}_{0.5} \mathrm{Nb}_{0.5} \mathrm{O}_{3}$ system would result in a conducting oxide, which provides a superior epitaxial relationship with the (001) $\mathrm{MgAl}_{2} \mathrm{O}_{4}$ substrate. In a more general sense, such a substitution provides a means for independently tuning the lattice parameter and conductivity over a significant range of values. Here, we report on the deposition of $\left(\mathrm{Ba}_{1-y} \mathrm{Sr}_{y}\right) \mathrm{Ti}_{0.5} \mathrm{Nb}_{0.5} \mathrm{O}_{3}$ thin films on (001) $\mathrm{MgAl}_{2} \mathrm{O}_{4}$ substrates for $y=0,0.4,0.5$, and 0.6. Characterization of these films using a variety of characterization techniques, including $\theta-2 \theta$ x-ray diffraction (XRD), 2-dimensional XRD (2D-XRD), scanning electron microcopy (SEM), and transmission electron microscopy (TEM), clearly demonstrate significant improvements to the film quality brought about by the strontium substitution.

\subsection{Phase purity and lattice parameters}

The crystallinity and phase purity for the $\left(\mathrm{Ba}_{1-y} \mathrm{Sr}_{y}\right) \mathrm{Ti}_{0.5} \mathrm{Nb}_{0.5} \mathrm{O}_{3}$ thin films were examined using XRD techniques. A Bruker D8 Advance powder diffractometer was used to perform $\theta-2 \theta$ XRD scans. The instrument was equipped with a $\mathrm{Cu}$ 
$\mathrm{K}_{\alpha 1}$ source and a germanium monochromator. Scans were conducted with a $0.03^{\circ}$ step size and a 4 second dwell time. It should be noted that this technique is only sensitive to periodicities normal to the substrate's surface. The data, presented in figure 9.1, all show a prominent (002) reflection from the film, a clear indication that it is composed of highly oriented material. The full width at half maximum (FWHM) of this peak is $0.28^{\circ}$ for the $y=0$ film and approximately $0.23^{\circ}$ for all other compositions. It should also be noted that with increasing $y$ the (002) reflection shifts to higher $2 \theta$ angles, indicating that the lattice parameter is, as expected, decreasing for compositions with higher strontium content. The corresponding outof-plane lattice parameters are listed in table 9.1. It is noticed that the $c=4.091 \AA$ of $y=0$ film here deposited at $850^{\circ} \mathrm{C}$ is a little bit smaller than $c=4.098 \AA$ which deposited at $790^{\circ} \mathrm{C}$ (see table 7.1 on page 95). A close inspection of the data also reveals the existence of a number of low intensity peaks. The film reflection near $69^{\circ}$, which is observed for all values of $y$ is difficult to assign as it could originate from either the (003) reflection or from both the (003) and (221) reflections. It should also be noted that the peaks corresponding to the (011) and (111) reflections (which are normal to the substrate surface) are strongest for the $y=0$ film, with no such peaks observed for the $y=0.5$ and $y=0.6$ compositions. These results suggest that the strontium doping has improved the crystallinity, inhibited the formation of alternate orientations, and reduced the mismatch to the underlying substrate. 


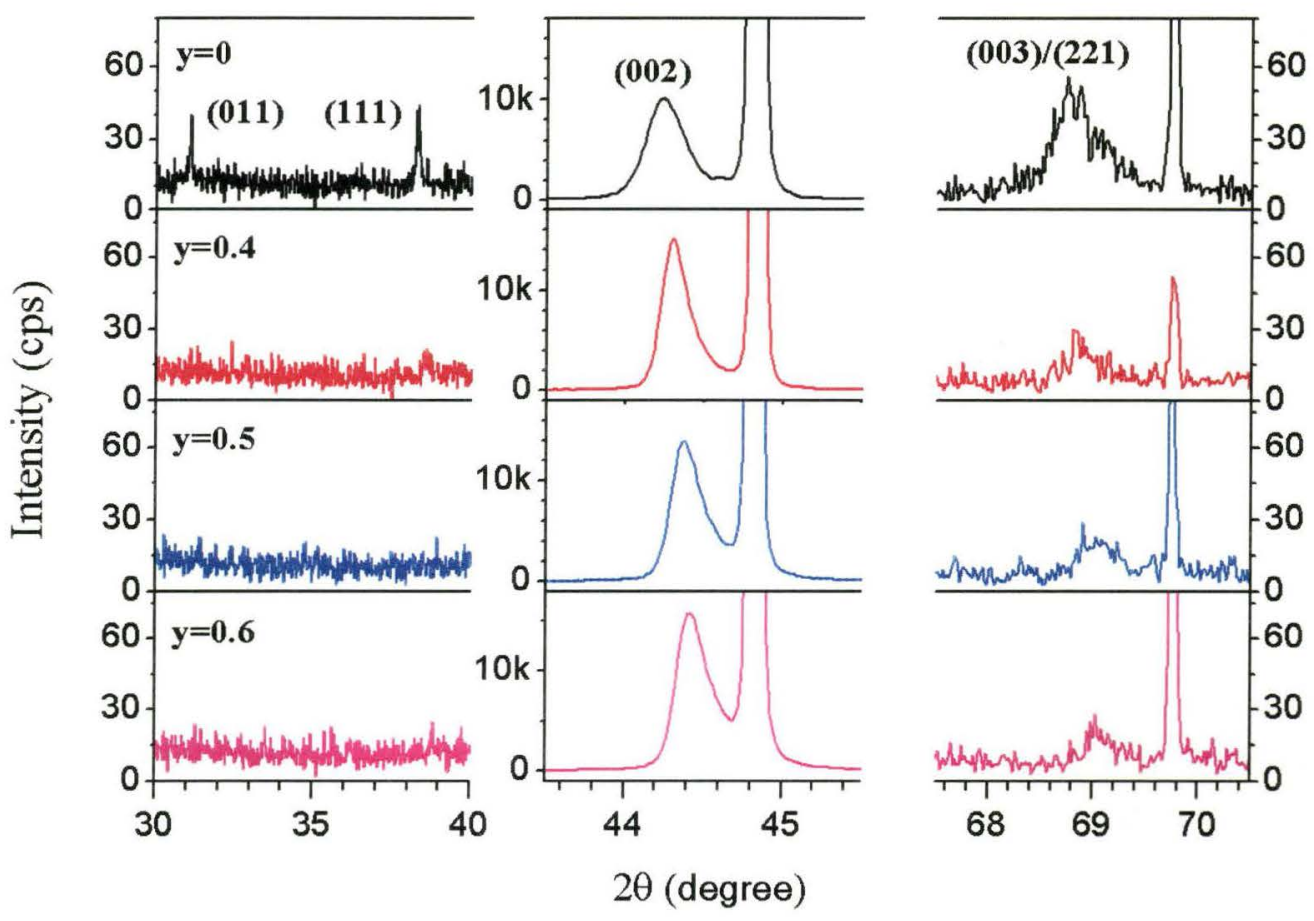

Figure 9.1: $\theta-2 \theta$ XRD data for $\mathrm{Ba}_{1-y} \mathrm{Sr}_{y} \mathrm{Ti}_{0.5} \mathrm{Nb}_{0.5} \mathrm{O}_{3}$ thin films with various values of $y$. All films exhibit a strong (002) reflection, but note that it shifts towards higher $2 \theta$ values as $y$ is increased. Also of significance is that the $y=0$ sample shows weak (011) and (111) reflections, while the high values of $y$ do not. All samples show a peak around $69^{\circ}$, which can be assigned to two reflections. For the purpose of clarity only select ranges of $2 \theta$ values are shown in the figure even though the scan was conducted over the interval between $15^{\circ}$ and $75^{\circ}$. The only film peak not shown in the figure is the (001) reflection. The sharp peak at $69.8^{\circ}$ is associated with the substrate's (004) reflection.

Table 9.1: The value of lattice parameter $c$ of $\left(\mathrm{Ba}_{1-y} \mathrm{Sr}_{y}\right) \mathrm{Ti}_{0.5} \mathrm{Nb}_{0.5} \mathrm{O}_{3}$

\begin{tabular}{ccc}
\hline$\left(\mathrm{Ba}_{1-y} \mathrm{Sr}_{y}\right) \mathrm{Ti}_{0.5} \mathrm{Nb}_{0.5} \mathrm{O}_{3}$ & 2theta value (degree) & Lattice parameter $c(\AA)$ \\
\hline $\mathrm{y}=0$ & $44.24(2)$ & $4.091(2)$ \\
$\mathrm{y}=0.40$ & $44.30(2)$ & $4.086(2)$ \\
$\mathrm{y}=0.50$ & $44.36(2)$ & $4.081(2)$ \\
$\mathrm{y}=0.60$ & $44.40(2)$ & $4.077(2)$ \\
\hline
\end{tabular}




\subsection{Grain orientation}

Additional structural information of $\left(\mathrm{Ba}_{1-y} \mathrm{Sr}_{y}\right) \mathrm{Ti}_{0.5} \mathrm{Nb}_{0.5} \mathrm{O}_{3}$ was obtained using 2D-XRD techniques. Such measurements, while of lower angular resolution, are superior to $\theta-2 \theta$ measurements in that it is not limited to the observation of periodicities which are normal to sample's surface, thus providing additional information such as the in-plane orientation of the film relative to the substrate. The data was collected by a three-circle Bruker D8 diffractometer with a 2D SMART6000 CCD detector. After processing the raw data, pole figures were generated using the GADDS software package. Such pole figures are merely stereographic maps of the film which pinpoint the real-space directions (i.e. poles) where specific periodicities lie. Thus, for a cubic film, a pole in the center of $\{001\}$ pole figure indicates that the $c$-axis periodicity is normal to the substrate $\left(\phi=0^{\circ}\right)$, while one at the edge of the pole figure $\left(\phi=90^{\circ}\right)$ indicates that it is parallel. Other orientations are represented by the intermediate values. From a practical point of view, the edges of the pole figure $\left(\phi>75^{\circ}\right)$ are experimentally inaccessible. Due to the close latticematch between the film and the substrate, pole figures showing periodicities from both materials is inevitable. Table 9.2 summarizes the extent of the overlap for the two pole figures presented here. In an ideal scenario, a single pole figure provides a complete understanding of the film's grain structure, but complications due to overlaps and experimentally inaccessible regions of the pole figure necessitate that two such figures be presented in this report if a complete understanding is to be obtained. 
Table 9.2: Summary of the observed reflections for the $2 \theta$ integration windows used in the generation of various pole figures. Because the generation of a pole figure requires a $2 \theta$ integration window, closely spaced periodicities will end up in the same pole figure. Thus, for the pole figures in this chapter, there are peaks corresponding to both film and substrate reflections.

\begin{tabular}{ccl}
\hline Pole figure & Integration range $(2 \theta)$ & Covered reflections \\
\hline$\{001\}$ & $42.3-46.7^{\circ}$ & substrate $(004)+$ film $(002)$ \\
$\{011\}$ & $30.2-32.0^{\circ}$ & substrate $(022)+$ film $(011)$ \\
$\{111\}$ & $36.0-38.0^{\circ}$ & substrate $(311)+$ film $(111)$ \\
\hline
\end{tabular}

The $\{111\}\left(\mathrm{Ba}_{1-y} \mathrm{Sr}_{y}\right) \mathrm{Ti}_{0.5} \mathrm{Nb}_{0.5} \mathrm{O}_{3}$ pole figure, shown in figure 9.2, was used to determine the epitaxial relationship between the film and substrate. While the $\{111\}$ reflections are weak, they are the only set of reflections where there is no significant overlap between the film and substrate peaks. The pole figure, which is essentially identical for all values of $y$, shows only four poles at $\phi=54.7^{\circ}$ separated by an azimuthal angle of $90^{\circ}$. This is the pattern expected for a [001] oriented film with a single in-plane orientation (i.e. the pole pattern expected for a single crystal). Likewise, the substrate shows the $\{311\}$ response expected of a single crystal. Thus, by comparing the relative azimuthal relationship between these two single crystal patterns the epitaxial orientation between the film and substrate is shown to be $(001)[100]_{\mathrm{film}} / /(001)[100]_{\text {substrate }}$. This relationship is exactly what is expected from lattice match considerations. While the $\{111\}\left(\mathrm{Ba}_{1-y} \mathrm{Sr}_{y}\right) \mathrm{Ti}_{0.5} \mathrm{Nb}_{0.5} \mathrm{O}_{3}$ pole figure can be used to determine the epitaxial relationship between the substrate and the dominant orientation of the film, the weakness of the $\{111\}$ reflections make them inadequate for observing small volumes of misoriented grains because the scattering from these structures is comparable to the noise level. These structures, however, can be observed using pole figures derived from X-ray reflections with larger structure factors such as those obtained from the $\{002\}$ and $\{011\}$ re- 


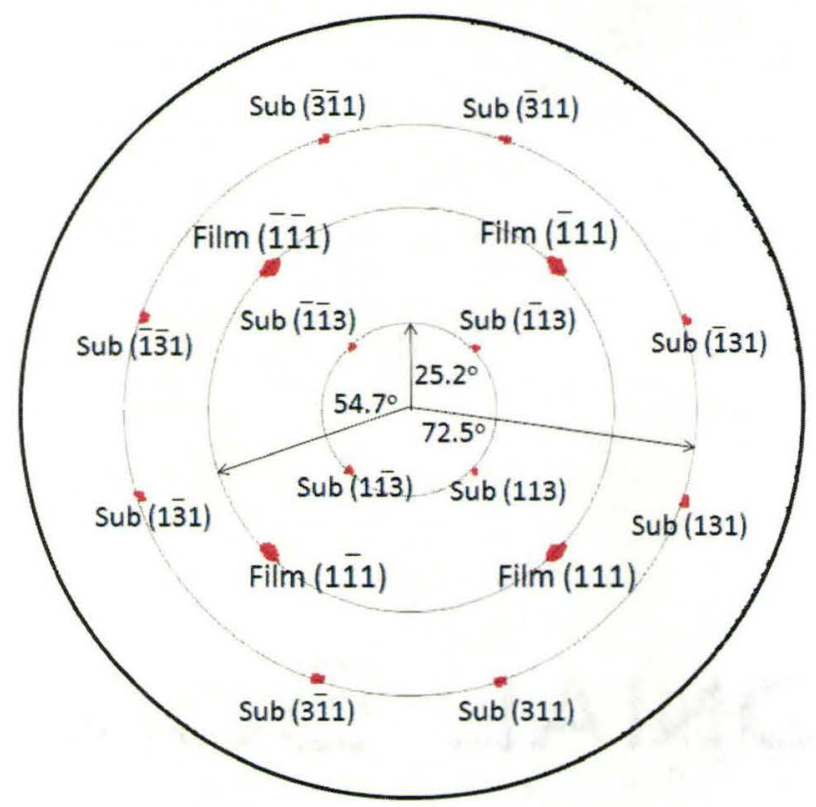

Figure 9.2: Pole figure of the $\{111\}$ film and $\{311\}$ substrate periodicities for the $y=0$ composition. Both the film and substrate show the stereographic projection expected of a [001] oriented single crystal with relative in-plane orientations as determined by the axes to the right of the figure. Of note, is that the $\{111\}$ film pole figures for all values of $y$ have the same appearance (not shown).

flections. For these pole figures, however, the poles from the dominant grain orientation are obscured by overlaps with those of the substrate.

Figure 9.3 shows the $\{001\}$ pole figures for the $y=0$ and 0.6 compositions. The $y=0.6$ composition shows only a single pole in the center of the pole figure, which originates from an overlap of the film's [002] and the substrate's [004] pole. The $y=0$ film, on the other hand, shows twelve weak poles in addition to the dominant [002] orientation represented by the central pole. These weak poles can be subdivided into groups of three (those connected by a triangle), where each group corresponds to a distinct grain orientation. The position of these peaks on 


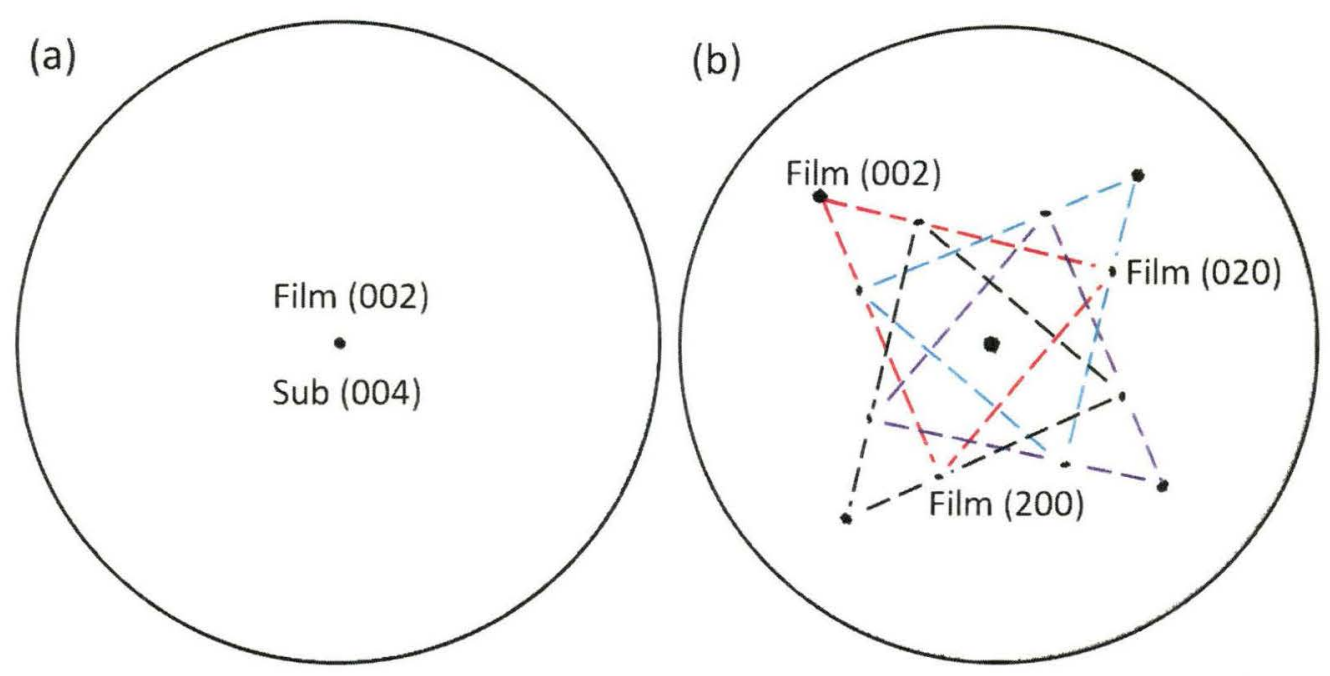

Figure 9.3: Pole figure of the $\{002\}$ film and $\{004\}$ substrate periodicities for the (a) $y=$ 0.6 and (b) $y=0$ compositions. Note that the $y=0.6$ composition shows only a single peak in the center of the pole figure, while the $y=0$ sample shows 12 weak poles characteristic of four [221] oriented grains with four distinct in-plane orientations. The poles arising from each [221] oriented grain are connected with lines to form a triangle.

the pole figure allow one to infer that all four of these grains are [221] oriented (i.e. the (221) planes are parallel to the substrate's surface), but with four different inplane orientations. The fact that these misorientated grains are present in the $y=0$ sample, but absent in all other compositions is further evidence of the superior film quality brought about through the strontium substitution. Of note, is that the small (011) and (111) peaks observed in the $\theta-2 \theta$ XRD scans are not observed in the $y=0$ pole figure. This indicates that these are minor fractions even when compared to the small amount of [221] oriented material present. Also, confirmed is a combined $(221) /(300)$ assignment for the $69^{\circ} \theta-2 \theta$ XRD peak, but there still remains some ambiguity as to whether there exist even smaller percentages of this [221] orientation for the $y>0$ compositions.

The results of all $\{001\}$ and $\{011\}$ pole figures of $\left(\mathrm{Ba}_{1-y} \mathrm{Sr}_{y}\right) \mathrm{Ti}_{0.5} \mathrm{Nb}_{0.5} \mathrm{O}_{3}$ thin 

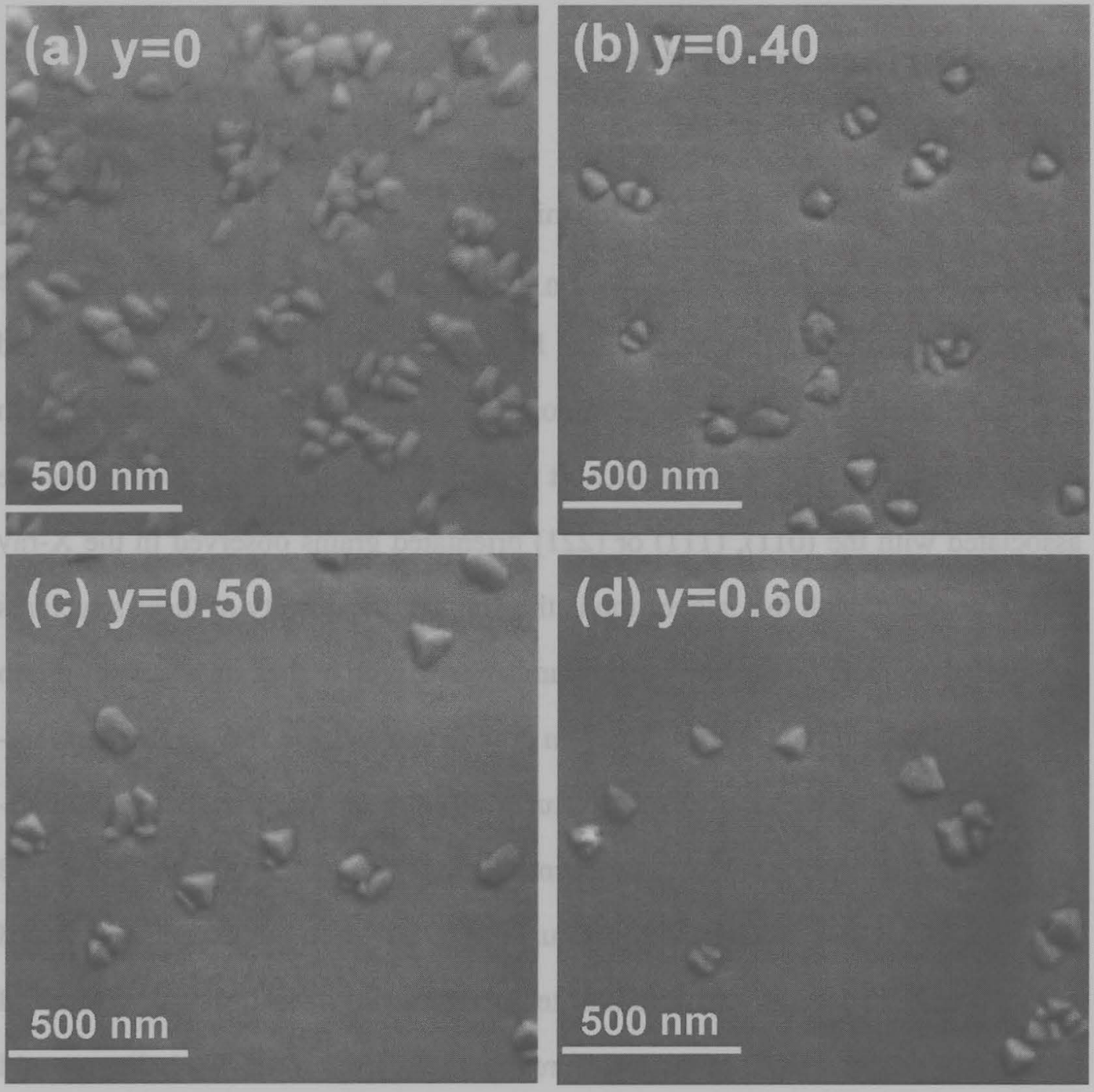

Figure 9.4: $\mathrm{SEM}$ images of $\left(\mathrm{Ba}_{1-y} \mathrm{Sr}_{y}\right) \mathrm{Ti}_{0.5} \mathrm{Nb}_{0.5} \mathrm{O}_{3}$ thin films deposited at $850^{\circ} \mathrm{C}$ for various values of $y$. Note that the surface morphology is significatly improved as the strontium content is increased.

films with $y=0,0.40 .5$ and 0.6 show in Appendix B. 


\subsection{Surface roughness}

A JEOL JSM-7000F scanning electron microscope (SEM), was used to examine the surface morphology of the $\left(\mathrm{Ba}_{1-y} \mathrm{Sr}_{y}\right) \mathrm{Ti}_{0.5} \mathrm{Nb}_{0.5} \mathrm{O}_{3}$ thin films. Figure 9.4 shows the images obtained for various values of $y$. In all cases, the surfaces are characterized by highly faceted protrusions superimposed on an extremely smooth layer which shows little in the way of granularity. It is highly likely that these protrusions are associated with the $(011),(111)$ or $(221)$ orientated grains observed in the X-ray data. Consistent with this suggestion is the fact that the density of these structures is highly dependent on the film's strontium content, with higher values of strontium yielding superior morphologies. The film growth temperature also significantly impacts their density, with higher temperatures yielding far fewer structures. Experimental limitations placed on the maximum substrate temperature allowed prevented film depositions at even higher temperatures. It is, however, our expectation, based on the established trend, that a further temperature rise of as little as $50^{\circ} \mathrm{C}$ could completely rid the surface of these features for the $y \geq 0.5$ films. Such films would meet the requirements of an oxide electrode material as they display excellent conductivity while showing the potential to form high quality heteroepitaxial interfaces.

\subsection{Deposition temperature effect}

Since the deposition temperature has a significant effect on the quality of films, this effect is studies by the $\left(\mathrm{Ba}_{1-y} \mathrm{Sr}_{y}\right) \mathrm{Ti}_{0.5} \mathrm{Nb}_{0.5} \mathrm{O}_{3}$ thin film of $\mathrm{y}=0.60$ due to its lowest 


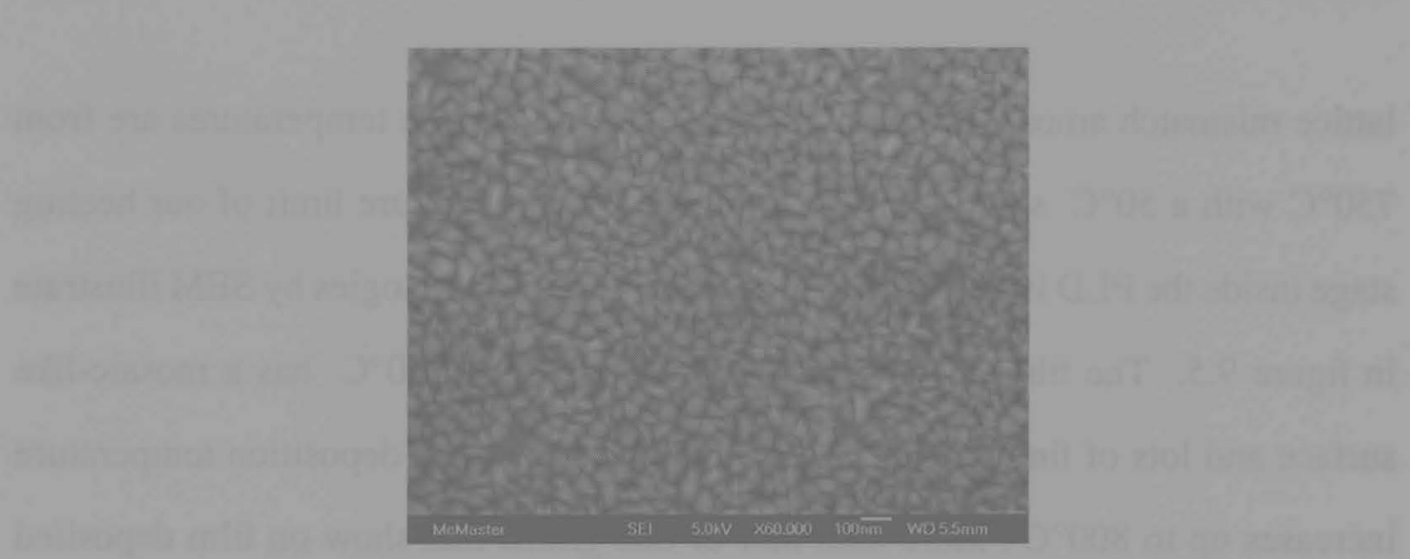

(a) $750^{\circ} \mathrm{C}$
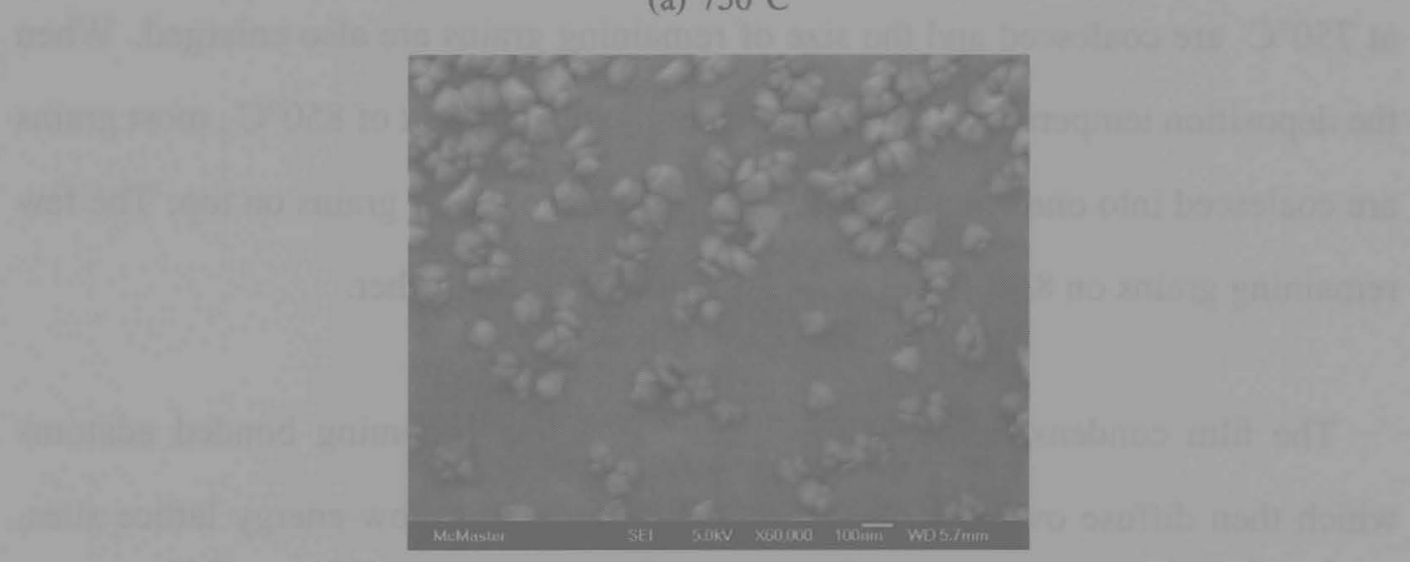

(b) $800^{\circ} \mathrm{C}$

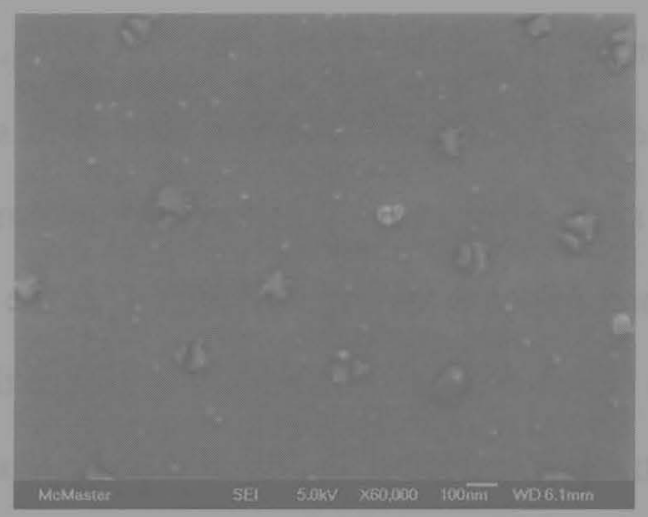

(c) $850^{\circ} \mathrm{C}$

Figure 9.5: $\mathrm{SEM}$ images of $\left(\mathrm{Ba}_{1-y} \mathrm{Sr}_{y}\right) \mathrm{Ti}_{0.5} \mathrm{Nb}_{0.5} \mathrm{O}_{3}$ thin film of $\mathrm{y}=0.60$ with different deposition temperature at (a) $750^{\circ} \mathrm{C}$, (b) $800^{\circ} \mathrm{C}$ and (c) $850^{\circ} \mathrm{C}$ 
lattice mismatch among our films. The selected deposition temperatures are from $750^{\circ} \mathrm{C}$ with a $50^{\circ} \mathrm{C}$ step to $850^{\circ} \mathrm{C}$, which is the temperature limit of our heating stage inside the PLD instrument. The result surface morphologies by SEM illustrate in figure 9.5. The film deposited at lower temperature $750^{\circ} \mathrm{C}$ has a mosaic-like surface and lots of fine grains of $20-30 \mathrm{~nm}$ on top. As the deposition temperature increases up to $800^{\circ} \mathrm{C}$, more than half of fine grains that show on film deposited at $750^{\circ} \mathrm{C}$ are coalesced and the size of remaining grains are also enlarged. When the deposition temperature pushes up to the instrument limit of $850^{\circ} \mathrm{C}$, most grains are coalesced into one continuous entity with few scattered grains on top. The few remaining grains on $850^{\circ} \mathrm{C}$ deposited film do not grow further.

The film condensation involves incoming atoms becoming bonded adatoms which then diffuse over the film surface until trapped at low-energy lattice sites, and such bonded adatoms reach their equilibrium position in lattices by bulk diffusion. Four basic processes are involves: (1) shadowing, (2) surface diffusion, (3) bulk diffusion, and (4) desorption. The first process arises from the geometric constraint due to the surface roughness of the growing film and the impingement direction. The last three process are directly related to the ability of diffusion or sublimation therefore the condensate melting point. Hence the dominant processes are a function of substrate temperature (deposition temperature), $\mathrm{T}_{S}$, by manifesting different structural morphologies.

The film surface morphology or structure dependent on deposition temperature effect has been well studied for evaporated metal films (Ohring, 2002). A structurezone diagrams (SZD) is also established (figure 9.6). According to the SZD, when 


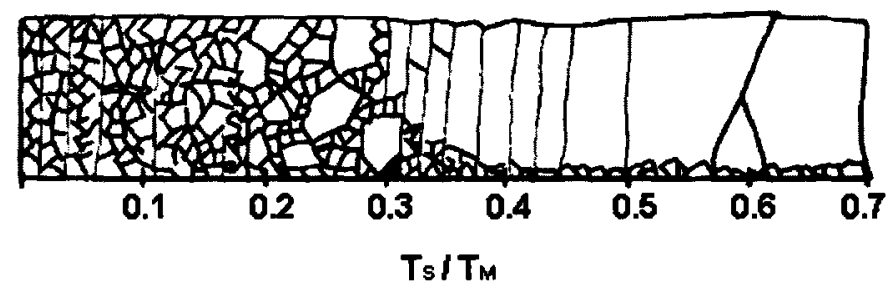

Figure 9.6: Structure zone diagram for evaporated metal films (Hentzell et al., 1984)

the ratio of $T_{S}$ (substrate temperature) over $T_{M}$ (condensate melting temperature) is higher than 0.5 , the granular structure will reduce. Although the melting points of $\left(\mathrm{Ba}_{1-y} \mathrm{Sr}_{y}\right) \mathrm{Ti}_{0.5} \mathrm{Nb}_{0.5} \mathrm{O}_{3}$ and $\mathrm{BaTi}_{1-x} \mathrm{Nb}_{x} \mathrm{O}_{3}$ thin films are unknown, the $1600^{\circ} \mathrm{C}$ for the melting point of $\mathrm{BaTiO}_{3}$ can be used as a reasonable guess. The corresponding value of $\mathrm{T}_{S} / \mathrm{T}_{M}$ is in the range of $0.55-0.60$ with respect to the substrate temperature range of $750-850^{\circ} \mathrm{C}$. The films surface morphologies (figure 9.5) are consistent in trend with the SZD prediction, but push the optimum $\mathrm{T}_{S} / \mathrm{T}_{M}$ to a higher value which is at least 0.60 . This deposition temperature effect should be valid for all our films regardless of $\mathrm{Sr}$ and $\mathrm{Nb}$ concentration, however, due to different lattice mismatch and possible variation of melting points, the optimum deposition temperature can be changed. Obviously, the surface morphology of (001) orienated $\mathrm{BaTi}_{0.5} \mathrm{Nb}_{0.5} \mathrm{O}_{3}$ thin film (figure 9.4 (a)) is not optimum, and several tens of degrees increase in deposition temperature seems necessary. However, limited by the heating stage of our instrument, such result is the most optimum one we can achieve. 


\subsection{Microstructure and interface analysis}

TEM cross-sections were used to examine the grain structure, crystallinity and the film-substrate interface for the $y=0.60\left(\mathrm{Ba}_{1-y} \mathrm{Sr}_{y}\right) \mathrm{Nb}_{0.5} \mathrm{Ti}_{0.5} \mathrm{O}_{3}$ film deposited at $850^{\circ} \mathrm{C}$. Figure $9.7(\mathrm{a})$ is a low magnification bright field (BF) image of the film's cross-section obtained using a Philips CM 12 TEM. The $200 \mathrm{~nm}$ thick film exhibits columnar bands with dark contrast, which extend from the substrate to the film's surface along a direction that is roughly perpendicular to the interface. The interface itself is sharp and flat. Figure 9.7(b) shows a selected area electron diffraction (SAD) pattern from a small area of the same cross-section which includes both sides of the interface. Such a pattern consists of a superposition of two subpatterns, with one originating from the film and the other from the substrate. Analysis of the diffraction pattern yields a film-substrate orientational relationship having $[001]_{\text {film }} / /[001]_{\text {substrate }}$ and $[100]_{\text {film }} / /[100]_{\text {substrate }}$, which is, as expected, identical to that obtained from the 2D-XRD analysis. Noteworthy, is the fact that the diffraction spots in the SAD pattern show no indication of splitting or any detectable misorientations, as is expected for closely matched film-substrate lattice parameters. This is in spite of the fact that columnar features are clearly visible in the BF image, a result suggestive of an extremely small tilt angle with a width of less than one SAD reflection. In fact, there does appear to be a barely visible peak splitting for the reflections at large scattering angles.

High resolution images of the interface, taken with a FEI Titan 80-300 Cubed, allow for an examination of film epitaxy. Figure 9.7(c) shows a high-resolution high-angle angular dark-field (HAADF) image of the interface. The contrast of 
the HAADF image is proportional to the scattering ability of the atoms (i.e. it is sensitive to the atomic number $(Z)$ ) and, as a consequence, light atoms show a lower intensity in the image than the heavier atoms. This fact allows for effective imaging of the interface as there exist substantial differences between the atomic numbers of the atoms in the substrate compared to those in the film. The imaged interface is sharp with the transition from substrate to film occurring within the width of one or two unit cells. The flawless alignment of atoms across the interface over the extent of the image is a clear indication of the high degree of epitaxy obtained.

\subsection{Hall coefficient measurement}

The Hall coefficient measurements here were carried out in order to understand the nature of charge carries and also their mobility. The room temperature Hall coefficient measurements were carried out by the ACCENT, HL5500 PC Hall Effect Measurement System under the DC mode with a 0.32 Tesla magnetic field. The experimental results were listed in the Table 9.3. The major charge carriers were electrons for all $\mathrm{Ba}_{1-y} \mathrm{Sr}_{y} \mathrm{Ti}_{0.5} \mathrm{Nb}_{0.5} \mathrm{O}_{3}$ films. The Hall mobilities and charge densities were also in the same order of those from $\mathrm{BaTi}_{0.5} \mathrm{Nb}_{0.5} \mathrm{O}_{3}$ thin film which were deposited at $790^{\circ} \mathrm{C}$ (see table 7.4 on page 125). Thus the Sr-doping doesn't significantly change the nature of charge carries or charge densities of $\mathrm{BaTi}_{0.5} \mathrm{Nb}_{0.5} \mathrm{O}_{3}$ films. 
(a)
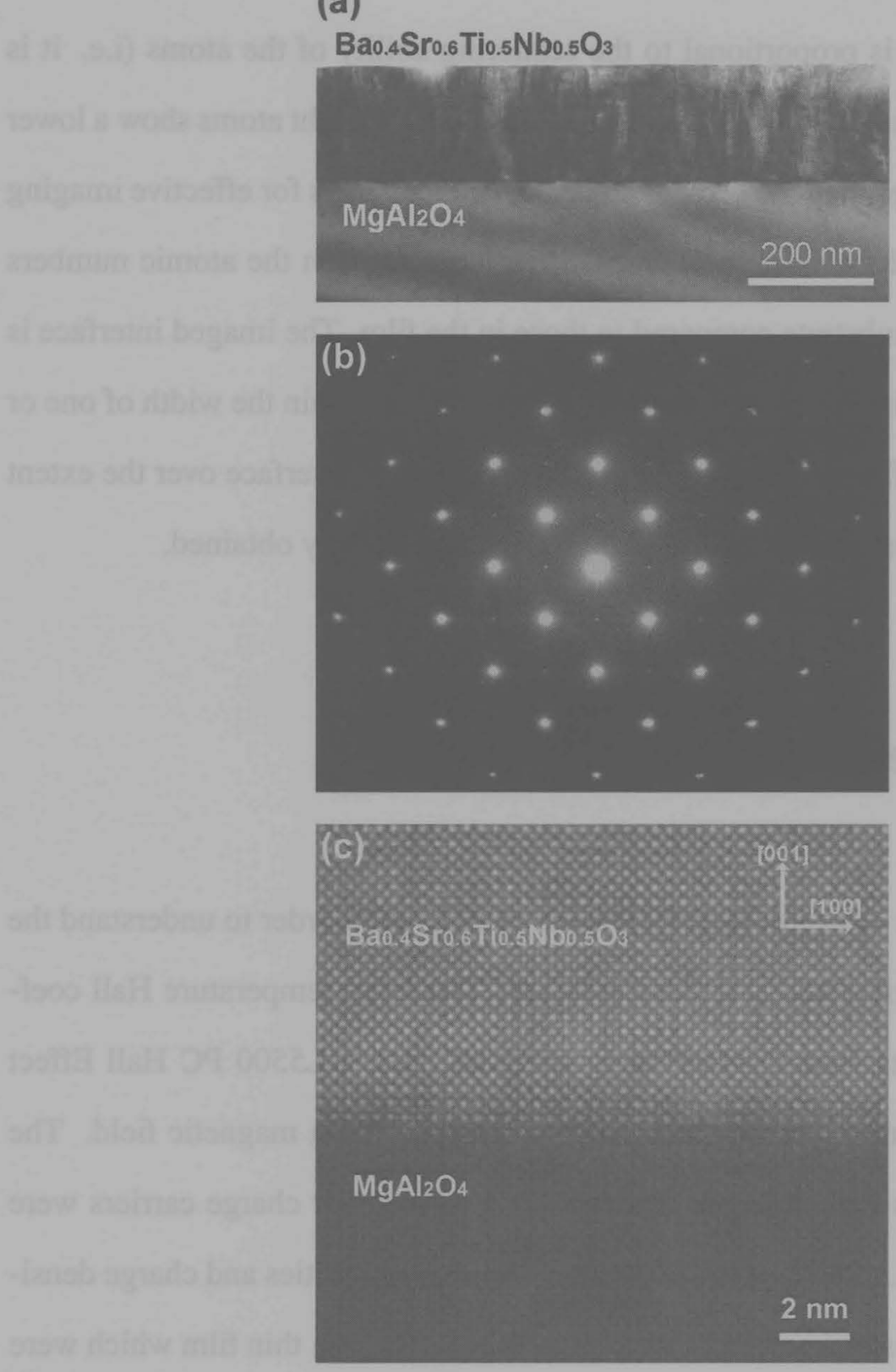

Figure 9.7: TEM results of $\left(\mathrm{Ba}_{1-y} \mathrm{Sr}_{y}\right) \mathrm{Ti}_{0.5} \mathrm{Nb}_{0.5} \mathrm{O}_{3}$ thin films (a) Low magnification bright field TEM image showing a $\left(\mathrm{Ba}_{0.4} \mathrm{Sr}_{0.6}\right) \mathrm{Nb}_{0.5} \mathrm{Ti}_{0.5} \mathrm{O}_{3}$ film-substrate combination in crosssection. (b) SAED pattern taken from an area spanning the interface. (c) High resolution HAADF image of the interface. All images were taken with the incident electron beam parallel to the substrate's [010] direction. Note the excellent epitaxial relationship formed between the film and substrate. 
Table 9.3: Hall effect measurements of $\left(\mathrm{Ba}_{1-y} \mathrm{Sr}_{y}\right) \mathrm{Ti}_{0.5} \mathrm{Nb}_{0.5} \mathrm{O}_{3}$

\begin{tabular}{lcclcc}
\hline Sample & $\rho_{R T}(\Omega \cdot \mathrm{cm})$ & Hall Coeff. $\left(\mathrm{m}^{2} / \mathrm{C}\right)$ & $\mu_{H}\left(\mathrm{~cm}^{2} / \mathrm{V} \cdot \mathrm{s}\right)$ & type & $\mathrm{n}\left(/ \mathrm{cm}^{3}\right)$ \\
\hline $\mathrm{y}=0$ & $3.31 \times 10^{-4}$ & $-2.73 \times 10^{-2}$ & 16.5 & $\mathrm{n}$ & $1.14 \times 10^{21}$ \\
$\mathrm{y}=0.40$ & $3.62 \times 10^{-4}$ & $-3.62 \times 10^{-2}$ & 20.0 & $\mathrm{n}$ & $8.63 \times 10^{20}$ \\
$\mathrm{y}=0.50$ & $3.70 \times 10^{-4}$ & $-1.90 \times 10^{-2}$ & 10.3 & $\mathrm{n}$ & $1.64 \times 10^{21}$ \\
$\mathrm{y}=0.60$ & $3.56 \times 10^{-4}$ & $-2.92 \times 10^{-2}$ & 16.4 & $\mathrm{n}$ & $1.07 \times 10^{21}$ \\
\hline
\end{tabular}

\subsection{Resistivity measurement}

Figure 9.8 shows the resistivity of $\left(\mathrm{Ba}_{1-y} \mathrm{Sr}_{y}\right) \mathrm{Ti}_{0.5} \mathrm{Nb}_{0.5} \mathrm{O}_{3}$ thin films as a function of temperature. If we are just satisfied with the statement that the metallic state means that $d \rho / d T>0$, the metal-insulator $(\mathrm{MI})$ transitions are noticed for all films. As the Sr concentration increase, the MI transition temperatures consistently increase as well as the residual resistivity $\rho_{0}$. The residual resistivity $\rho_{0}$ is a measure of the degree of elastic scattering due to distortion or defects. Since those films are deposited at the same condition and the film quality improves as $\mathrm{Sr}$ doping, then the increased $\rho_{0}$ mainly contributes from the lattice distortion. This is consistent with the face that the lattice becomes unstable when $\mathrm{Sr}$ ions with smaller radii size (118 pm) substitute Ba ions (135 pm). However, this explanation is only qualitative, a quantitative explanation of the residual resistivity $\rho_{0}$ requires defects free single crystal samples.

The high-temperature resistivities of the samples (above the MI transition temperature) follow a power-law relation

$$
\rho(T)=\rho_{0}+A T^{2}
$$




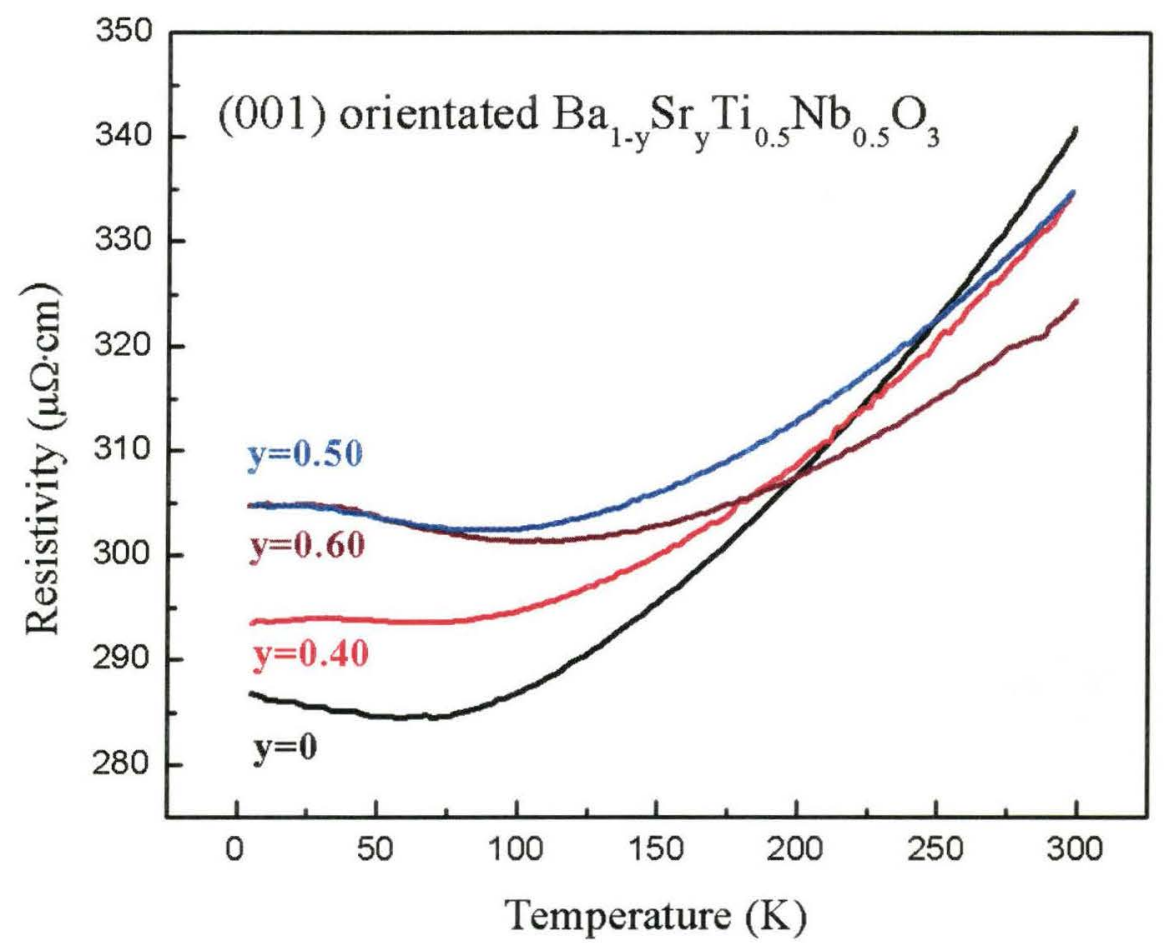

Figure 9.8: Resistivity of $\left(\mathrm{Ba}_{1-y} \mathrm{Sr}_{y}\right) \mathrm{Ti}_{0.5} \mathrm{Nb}_{0.5} \mathrm{O}_{3}$ thin films as a function of temperature where $\rho_{0}$ is the residual resistivity and $\mathrm{A}$ is constant, and the fitted results are shown in figure 9.9. Each samples perfectly fits to the power-law relation, and implies electron-electron interactions dominate over any type of electron scattering about the MI transition temperature. While the upturn in $\rho$ at low temperatures could be an evidence of quantum interference effects in electronic conduction such as weak localization effects (see section 2.5.4 on page 21) in our metallic thin films.

In summary, epitaxial (001) oriented metallic oxide $\left(\mathrm{Ba}_{1-y} \mathrm{Sr}_{y}\right) \mathrm{Ti}_{0.5} \mathrm{Nb}_{0.5} \mathrm{O}_{3}$ films with $y=0,0.4,0.5$ and 0.6 , are deposited onto (001) $\mathrm{MgAl}_{2} \mathrm{O}_{4}$ substrates using the pulsed laser deposition technique. The strontium for barium substitution 

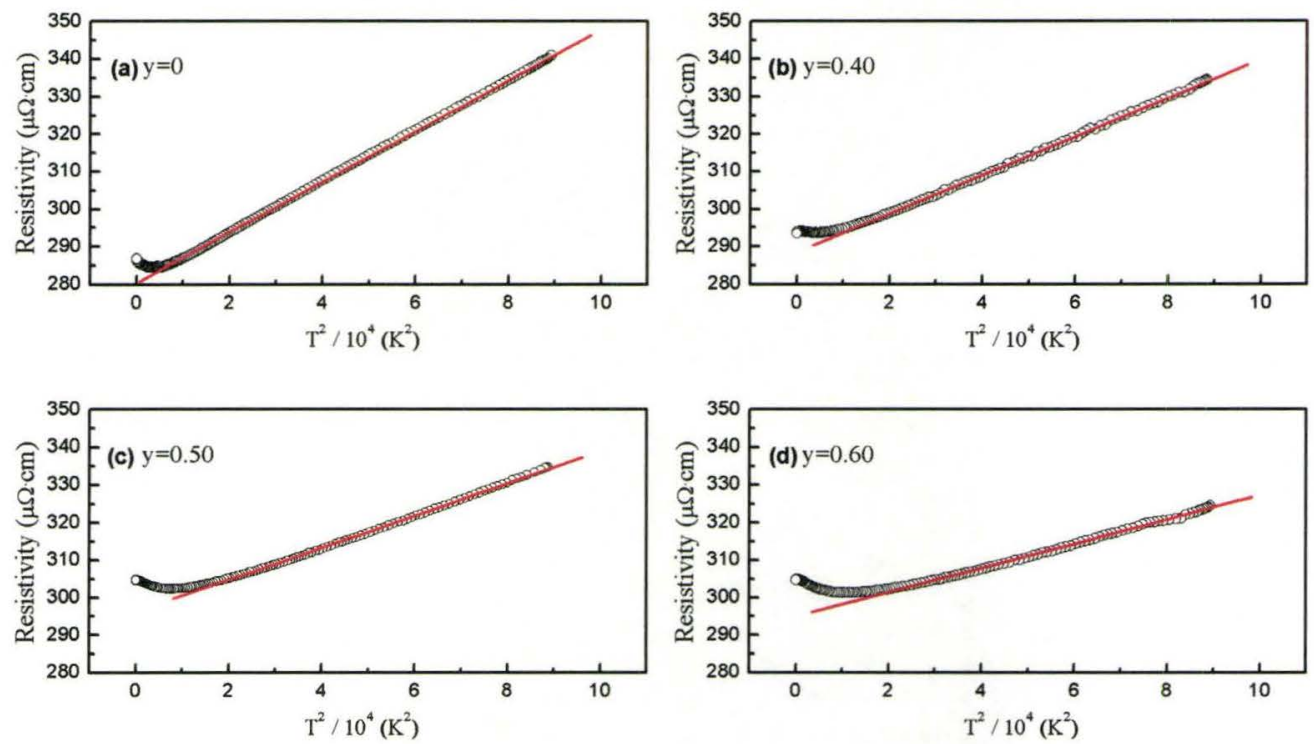

Figure 9.9: Temperature dependences of resistivity plot vs $\mathrm{T}^{2}$ of $\left(\mathrm{Ba}_{1-y} \mathrm{Sr}_{y}\right) \mathrm{Ti}_{0.5} \mathrm{Nb}_{0.5} \mathrm{O}_{3}$ thin films for (a) $y=0$, (b) $y=0.40$, (c) $y=0.50$ and (d) $y=0.60$. Symbols are experimental data and lines are results of the fit $\rho(T)=\rho_{0}+A T^{2}$

gives rise to a decrease in the lattice constant, without altering the conductivity of the film. X-ray diffraction measurements show a significant number of [111], [011] and [221] misoriented grains, that are not present for the $y=0.6$ composition. Transmission electron microscopy images of the film-substrate interface obtained for the $y=0.6$ composition show a sharp interface with flawless epitaxy. We attribute the improvements to a decrease in the lattice misfit strain made possible through the superior lattice match to the substrate obtained through strontium substitution. The electron-electron interaction dominates the metallic conducting behaviors of the $\left(\mathrm{Ba}_{1-y} \mathrm{Sr}_{y}\right) \mathrm{Ti}_{0.5} \mathrm{Nb}_{0.5} \mathrm{O}_{3}$ thin films when the temperature is higher than $100 \mathrm{~K}$. 


\section{Chapter 10}

\section{Quantification of the Ti oxidation}

\section{state}

The study of the transition-metal oxides is one of the central topics in condensed matter physics for decades due to their complex electronic structures and rich optical and electronic properties. The unusual properties of the transition metal oxides are believed to be due to their unique nature of the outer $d$ electrons (Cox, 1992; Rao \& Raveau, 1995) . Electron energy loss spectroscopy is an ideal tool to investigate the unoccupied state in the materials and thus the valence information or $d$ band occupancy of the $3 d$ transition-metals. For the $3 d$ transition metals, the $3 d$ occupancy is progressively increased across the series. A relationship between the $3 d$ occupancy and the EELS white-line intensity has been established in both a series of $3 d$ transition metals (Pearson et al., 1993) and a series of $3 d$ transition metal oxides (Graetz et al., 2004). In addition, a 3d occupancy in one $3 d$ transition 
metal oxide can also be different due to the mixture of different valencies. Such a valence difference of a specific $3 d$ transition metal is important and sometimes crucial in determining the transport properties of materials (Coey et al., 1999; Varma, 1976; Butera et al., 2001). However, the mixed-valence is difficult to determine and is generally assumed from the charge balance based on the chemical composition which is not always correct, particularly if multiple valence is possible. In this chapter, we focused on a series of mixed-valence compounds which have the similar structure. A series of $\mathrm{BaTi}_{1-x} \mathrm{Nb}_{x} \mathrm{O}_{3}$ samples were selected for $\mathrm{Ti}^{4+} / \mathrm{Ti}^{3+}$ mixture not only because they can form a large range of solid solution in term of the valence change, but also because they have a relatively simple $3 d$ electron configuration as compared to the elements in the middle or late of $3 d$ series. From the material point of view, these $\mathrm{BaTi}_{1-x} \mathrm{Nb}_{x} \mathrm{O}_{3}$ material demonstrated a metal-insulator transition as the $\mathrm{Nb}$ content increases (Marucco et al., 1997; Liu et al., 2005; Kolodiazhnyi \& Wimbush, 2006), and could be used as the conducting oxide electrode material to facilitate an epitaxial deposition with the technologically important $\mathrm{BaTiO}_{3}$ and $\mathrm{Pb}\left(\mathrm{Zr}_{1-x} \mathrm{Ti}_{x}\right) \mathrm{O}_{3}$ materials. A series of $\mathrm{Ti}-\mathrm{L}_{2,3}$ edge and $\mathrm{O}-\mathrm{K}$ edge of $\mathrm{BaTi}_{1-x} \mathrm{Nb}_{x} \mathrm{O}_{3}$ samples were obtained (see section 7.7 on page 108) and quantitative EELS analysis on $\mathrm{Ti}-\mathrm{L}_{2,3}$ edges is performed here to retrieve the fractions of $\mathrm{Ti}^{4+}$ and $\mathrm{Ti}^{3+}$ by fitting the experimental EELS spectra to the reference spectra of materials with known valence.

The samples we used here are ceramic $\mathrm{BaTi}_{1-x} \mathrm{Nb}_{x} \mathrm{O}_{3}$ samples and thin film $\mathrm{BaTi}_{0.5} \mathrm{Nb}_{0.5} \mathrm{O}_{3}$ (see section 7.7.1 on page 108 and chapter 5 on page 81). EEL spectra were collected on the FEI Titan 80-300 with a monochromator and energy loss spectrometer (Gatan 865 model). The energy spread of the incident beam in a 
single spectrum taken with a $1 \mathrm{~s}$ exposure time at FWHM was $0.24-0.27 \mathrm{eV}$. The spatial resolution of our experiment is around a few hundred nanometers to ensure a reasonable statistic of the mixed valences while still ensuring the film is sampled. The spectra presented here are a summation of individual spectra taken with an exposure time of $20 \mathrm{~s}$ each and an energy dispersion of $0.03 \mathrm{eV} /$ Channel. The individual spectra were offset from each other by $1-10 \mathrm{eV}$ with respect to each other by varying the drift tube energy. They was then manually realigned and added together during post-processing. This process allows efficient averaging of spectral noise and CCD artifacts so as to achieve long exposure times free from cumulative instrumental energy drift effects. Absolute measurement of the energy scale requires precise alignment of the zero loss peak (ZLP) and careful monitoring of the energy drift during data recording. The absolute energy scale was not required for this work as we used the shape of the spectra as reference in the film. The core-loss features on the Ti- $\mathrm{L}_{2,3}$ and $\mathrm{O}-\mathrm{K}$ edges did not change with exposure time and thus it can reasonably be assumed that no noticeable beam damage occurred in the area of interest.

Usually, the ratio of the integrated intensities of the $\mathrm{L}_{3}$ and $\mathrm{L}_{2}$ white line $\left(\mathrm{L}_{3} / \mathrm{L}_{2}\right)$ can be correlated to the valency of the transition metal atoms in ternary transition metal oxides (Rask et al., 1987; Paterson \& Krivanek, 1990), and the $L_{3} / L_{2}$ ratio and normalized white line intensity can be used to extract the valency (Botton et al., 1995). However, for the early $3 d$ transition metals, such as $\mathrm{Ti}$, the $2 p$ spin orbit coupling is not strong enough to completely separate the $L_{2}$ and $L_{3}$ peaks, and the multiplet effect can also cause the overlapping transitions from $2 \mathrm{p}_{1 / 2}$ and $2 \mathrm{p}_{3 / 2}$ states (Zaanen et al., 1985b). Although gaussian functions can be used to separate 
the $L_{2}$ and $L_{3}$ peaks by fitting the experimental spectra obtained with an energy resolution of $1.5 \mathrm{eV}$ (Graetz et al., 2004), for higher energy resolution spectra more detail showing features in the Ti $L_{2,3}$ edge, it is difficult to separate the $L_{2}$ and $L_{3}$ peaks by simply fitting gaussian functions.

In order to extract the valence information, the experimental result of the Ti$\mathrm{L}_{2,3}$ edge from polycrystalline $\mathrm{BaTi}_{0.998} \mathrm{Nb}_{0.002} \mathrm{O}_{3}$ sample was used here as the reference spectrum of $\mathrm{Ti}^{4+}$, representing the characteristic of a $d^{0}$ transition metal in octahedral (or slightly distorted octahedral) symmetry; and the experimental result of the Ti-L $\mathrm{L}_{2,3}$ edge from $\mathrm{BaTi}_{0.5} \mathrm{Nb}_{0.5} \mathrm{O}_{3}$ thin film was used here as the reference spectrum of $\mathrm{Ti}^{3+}$ (Shao et al., 2008), representing a trait of a $d^{1}$ transition metal in Octahedral symmetry. The valence information of the other $\mathrm{BaTi}_{1-x} \mathrm{Nb}_{x} \mathrm{O}_{3}$ polycrystalline samples can then be extracted by other means as suggested below.

To eliminate artifacts, each spectrum of $\mathrm{Ti}-\mathrm{L}_{2,3}$ is processed by first removing the background (using a standard power law fitting) and then the Hartree-Slater modeled L edge atomic cross section from the Ti- $\mathrm{L}_{2,3}$ edge. This was done by first aligning the cross section on set maximum of $e_{g}$ peak for both $L_{3}$ and $L_{2}$ edges, and then a 30 point-weighted (about $1 \mathrm{eV}$ in energy scale) adjacenced-averaging method was applied to smooth the edge of the Hartree-Slater cross section.

In this work we employ the method described by Cressey et al. (1993) (used in X-ray Absorption Spectroscopy) to extract the fractions of different Ti valence components by a linear profile combination of $\mathrm{Ti}^{4+}$ and $\mathrm{Ti}^{3+}$ reference spectra (labeled in figure 10.1 ) from cerami $\mathrm{BaTi}_{0.998} \mathrm{Nb}_{0.002} \mathrm{O}_{3}$ and $\mathrm{BaTi}_{0.5} \mathrm{Nb}_{0.5} \mathrm{O}_{3}$ thin film respectively. This profile fitting method worked quite well for the $\mathrm{Cu}_{1.2} \mathrm{Mn}_{1.8} \mathrm{O}_{4}$ 


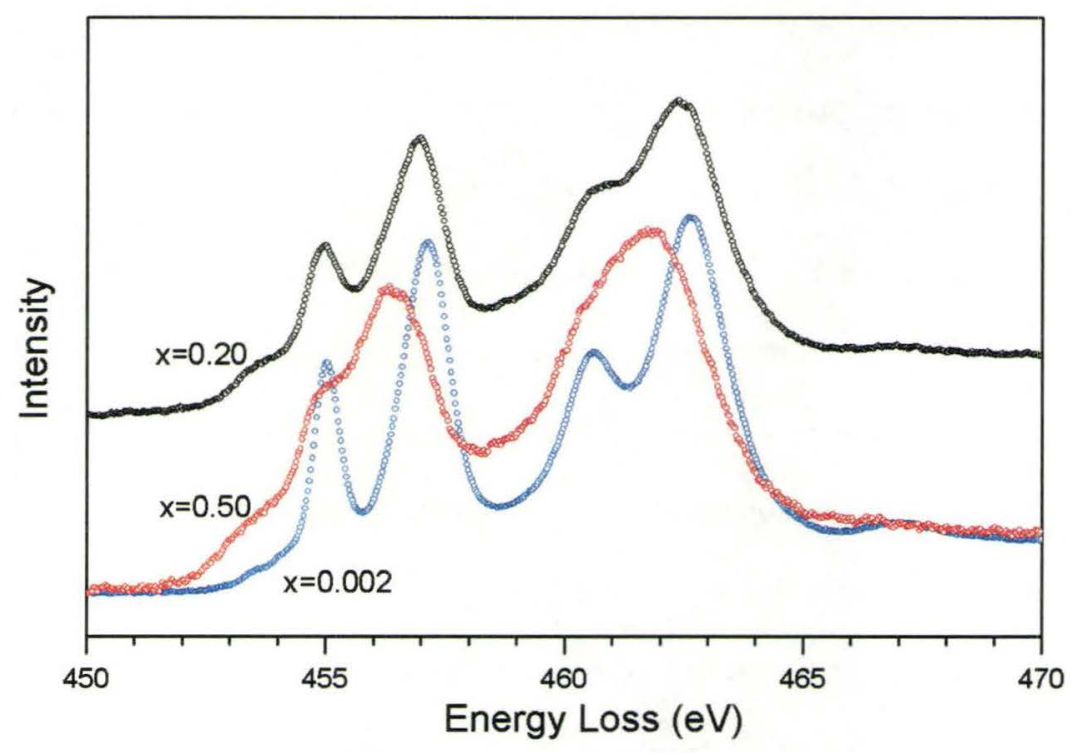

Figure 10.1: $\mathrm{Ti} \mathrm{L} 2,3$ edges in the reference compounds (polycrystalline $\mathrm{BaTi}_{0.998} \mathrm{Nb}_{0.002} \mathrm{O}_{3}$ and thin film $\mathrm{BaTi}_{0.5} \mathrm{Nb}_{0.5} \mathrm{O}_{3}$ ) with $x=0.20$ sample after deconvolution, and subtraction of the background and atomic across section.

system (Maunders et al., 2008) and Fe minerals (Cressey et al., 1993).

In accordance with the X-ray absorption spectra of (de Groot et al., 1990), the energy of the $L_{3} t_{2 g}$ maximum of the each spectrum was aligned to $456 \mathrm{eV}$ except the $\mathrm{Ti}^{3+}$ reference spectrum which doesn't have an obvious $t_{2 g}$ and $\mathrm{e}_{g}$ split and was aligned to $457.5 \mathrm{eV}$ according to the maximum of its $\mathrm{L}_{3}$ peak. The absolutely value of the position of each spectrum is not corrected here as a free parameter.

In order to obtain the $\mathrm{Ti}^{4+}$ and $\mathrm{Ti}^{3+}$ reference spectra for fitting a shape of the spectrum, the polycrystalline $\mathrm{BaTi}_{0.998} \mathrm{Nb}_{0.002} \mathrm{O}_{3}$ sample and $\mathrm{BaTi}_{0.5} \mathrm{Nb}_{0.5} \mathrm{O}_{3}$ thin film are used as end point reference for $\mathrm{Ti}^{4+}$ and $\mathrm{Ti}^{3+}$ respectively to avoid the change in width of the peaks due to the different lattice distortions. To quan- 
tify the ratio of the total white line intensity $\mathrm{I}\left(\mathrm{L}_{3}\right)+\mathrm{I}\left(\mathrm{L}_{2}\right)$ of $\mathrm{Ti}^{4+}$ and $\mathrm{Ti}^{3+}$, a 0.1 eV/Channel energy dispersion was also used on the acquisition of the spectra for the powder $\mathrm{BaTiO}_{3}$ and $\mathrm{BaTi}_{0.5} \mathrm{Nb}_{0.5} \mathrm{O}_{3}$ thin film samples to cover the high energy part after the Ti-L2,3 edge, and a corresponding lower loss energy spectrum was also recorded for the deconvolution. Generally, the total while line intensity was normalized with respect to a $50 \mathrm{eV}$ wide, continuum window $50 \mathrm{eV}$ above the $\mathrm{L}_{3}$-edge onset (Pearson et al., 1993; Graetz et al., 2004). However, due to the short energy separation between Ti- $\mathrm{L}_{2,3}$ edge (around $460 \mathrm{eV}$ ) and the following $\mathrm{O}-\mathrm{K}$ edge (around $530 \mathrm{eV}$ ), the $50 \mathrm{eV}$ continuum window overlapped with the $\mathrm{O}$ $\mathrm{K}$ edge. To avoid the overlapping, a $20 \mathrm{eV}$ continuum window was used here to quantify the ratio of the total white line intensities of $\mathrm{Ti}^{4+}$ and $\mathrm{Ti}^{3+}$. According to the one-electron model, the intensity of a transition from $|2 p\rangle$ state to an excited $\| 3 d\rangle$ state was controlled by the inelastic scattering factor and also the density of the unoccupied state $\rho^{u}(3 d)$. The total intensity in the edge can be expressed as equation 10.1:

$$
I \propto \rho^{u}(3 d)|\langle 3 d|\exp (i q \cdot r)| 2 p\rangle|^{2}
$$

The total white line intensity is the sum of all the transitions within a given energy region $\left(E_{0}-E_{1}\right)$. In this experiment, the white-line intensity was normalized to the $20 \mathrm{eV}$ continuum window:

$$
I \approx \rho^{u}(3 d) \frac{\int_{E_{0}}^{E_{1}}|\langle 3 d|\exp (i q \cdot r)| 2 p\rangle|^{2}}{\int_{E_{0}+50}^{E_{0}+70}|\langle 3 d|\exp (i q \cdot r)| 2 p\rangle|^{2}}=\rho^{u}(3 d) \frac{M_{\text {white line }}}{M_{\text {continuum }}}
$$

Before normalization, the spectrum has to be deconvoluted with the corresponding low loss spectrum at first to remove possible multiple inelastic scattering losses, 


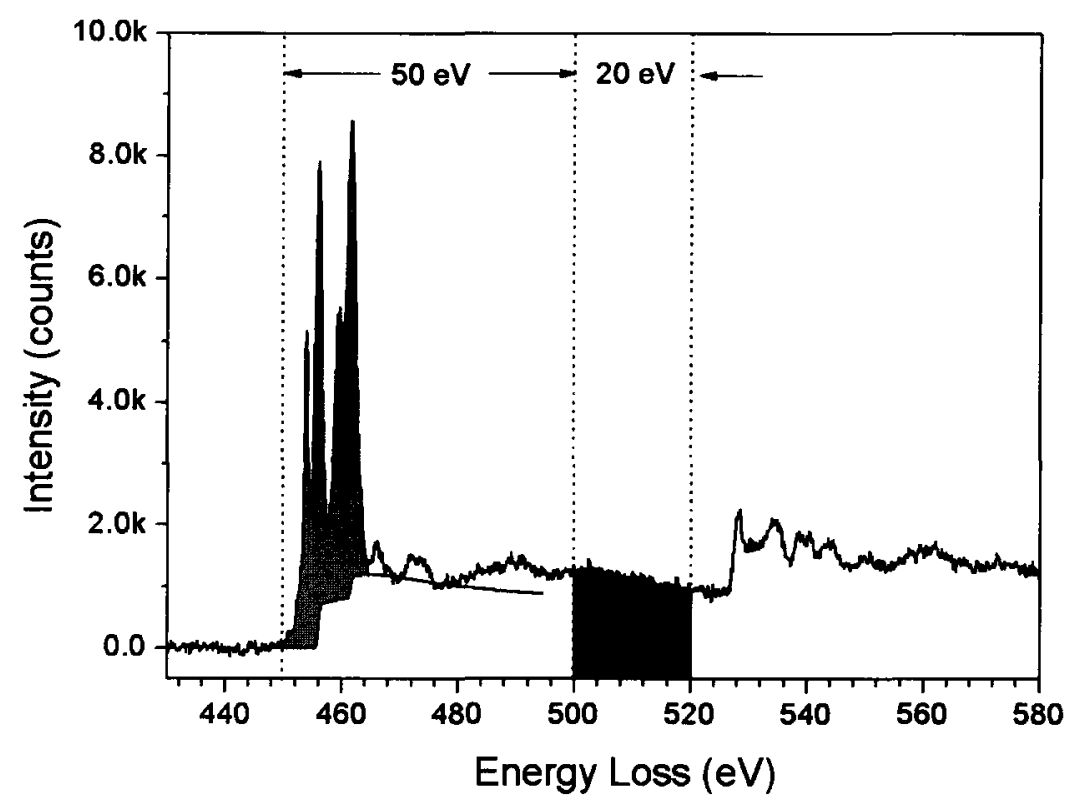

Figure 10.2: $\mathrm{Ti}-\mathrm{L}_{2,3}$ edge from $\mathrm{BaTiO}_{3}$ showing the method for isolating and normalizing the white-line intensity after deconvolution and standard background subtraction. Using this method, the real ratio of the total white line intensities of $\mathrm{Ti}^{4+}$ and $\mathrm{Ti}^{3+}$ is obtained as 1:1.07 for $\mathrm{BaTiO}_{3}$ and $\mathrm{BaTi}_{0.5} \mathrm{Nb}_{0.5} \mathrm{O}_{3}$.

the background needs to be subtracted in the power law, and atomic cross section needs to be removed. Using $\mathrm{BaTiO}_{3}$ as an example, this method is illustrated in 10.2. Our experimental results show a ratio of the total white line intensities of $\mathrm{Ti}^{4+}$ and $\mathrm{Ti}^{3+}$ to be $1: 1.07$, which is significantly deviated from the ratio of 10:9 based on the number of $3 d$ holes or the density of state of the unoccupied state $\rho^{u}(3 d)$ expected from the purely ionic format valence. This difference suggest a different matrix-element correction factors, i.e. $M_{\text {white line }} / M_{\text {continuum }}$, for the $\mathrm{Ti}^{4+}\left(\mathrm{d}^{0}\right)$ and $\mathrm{Ti}^{3+}\left(\mathrm{d}^{1}\right)$. We use this ratio value to normalize the $\mathrm{Ti}^{4+}$ and $\mathrm{Ti}^{3+}$ reference spectra with an energy dispersion of $0.03 \mathrm{eV} /$ Channel.

The Ti- $\mathrm{L}_{2,3}$ edge from $\mathrm{BaTi}_{1-x} \mathrm{Nb}_{x} \mathrm{O}_{3}$ polycrystalline samples and the reference 
compounds polycrystalline $\mathrm{BaTi}_{0.998} \mathrm{Nb}_{0.002} \mathrm{O}_{3}\left(\mathrm{Ti}^{4+}\right)$ and $\mathrm{BaTi}_{0.5} \mathrm{Nb}_{0.5} \mathrm{O}_{3}\left(\mathrm{Ti}^{3+}\right)$ are shown in figure 10.3. In order to compare the detailed features of the Ti- $\mathrm{L}_{2,3}$ edge, all spectra are aligned and scale to the total white line intensity as normalized to 1 . A clear trend is observed for samples with different $\mathrm{Nb}$ content. The four prominent peaks, $\mathrm{L}_{3}, \mathrm{~L}_{2}$ edge $\mathrm{t}_{2 g}$ and $\mathrm{e}_{g}$, for the low $\mathrm{Nb}$ content samples are gradually transformed into two very broad peaks with noticeable shoulders for the $x=0.50$ reference sample. The most significant effect is that all the peaks become broader, especially for the sharp $t_{2 g}$ peak shrinking to a broad shoulder. Due to the large lifetime broadening, the change of the $t_{2 g}$ of $L_{2}$ edge is more significant than that of $\mathrm{L}_{3}$ edge. The peak broadening can be explained in crystal field multiplet effect: the original 7 possible final states for $\mathrm{Ti}^{4+}\left(2 \mathrm{p}^{6} 3 \mathrm{~d}^{0} \rightarrow 2 \mathrm{p}^{5} 3 \mathrm{~d}^{1}\right)$ are replaced by 81 possible final states for the $\mathrm{Ti}^{3+}\left(2 \mathrm{p}^{6} 3 \mathrm{~d}^{1} \rightarrow 2 \mathrm{p}^{5} 3 \mathrm{~d}^{2}\right)$ in $\mathrm{O}_{h}$ symmetry (figure 7.15) although most of them have little intensity.

The fraction of each $\mathrm{Ti}^{4+}$ and $\mathrm{Ti}^{3+}$ components in the $\mathrm{BaTi}_{1-x} \mathrm{Nb}_{x} \mathrm{O}_{3}(0<x \leq$ 0.2 ) is extracted by the profile fitting procedure described above. For each sample, the best fit is achieved by using the scaling factors listed in the table 10.1, of the $\mathrm{Ti}^{4+}$ and $\mathrm{Ti}^{3+}$ reference spectra respectively. The unique profile fitting result was estimated by the least square method. The fitting was very good with all the features being reproduced accurately and demonstrating a reasonable $\mathbf{R}^{2}$ value. Although due to the overlap of the $\mathrm{L}_{3}$ and $\mathrm{L}_{2}$ peaks an accurate ratio of $\mathrm{L}_{3} / \mathrm{L}_{2}$ could not be obtained, the linear profile fitting procedure ensured that both the detailed shape and $\mathrm{L}_{3} / \mathrm{L}_{2}$ ratio are satisfied at the same time. 


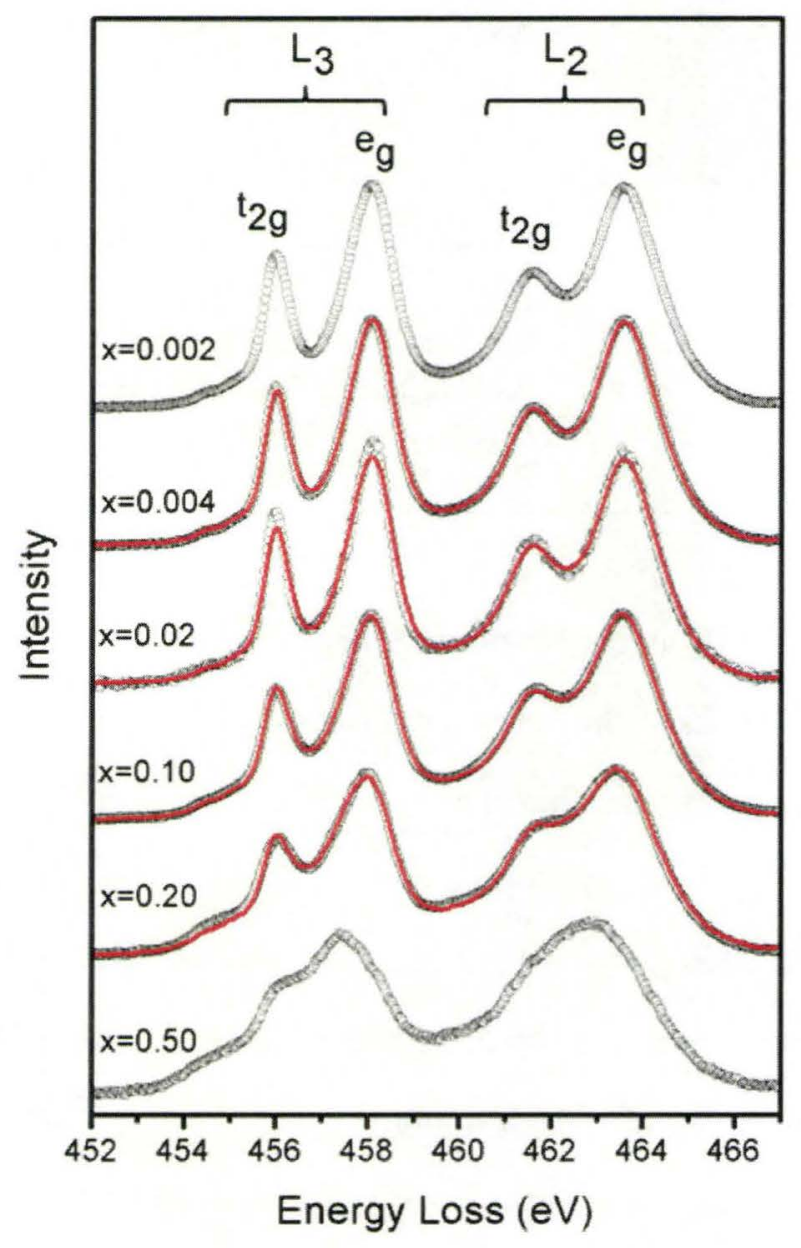

Figure 10.3: The Ti- $\mathrm{L}_{2,3}$ edge from $\mathrm{BaTi}_{1-x} \mathrm{Nb}_{x} \mathrm{O}_{3}$ polycrystalline samples and the reference compounds $\mathrm{BaTi}_{0.998} \mathrm{Nb}_{0.002} \mathrm{O}_{3}\left(\mathrm{Ti}^{4+}\right)$ and $\mathrm{BaTi}_{0.5} \mathrm{Nb}_{0.5} \mathrm{O}_{3}\left(\mathrm{Ti}^{3+}\right)$. The circles are the experimental results and the red solid lines are the profile fitting results with reference spectra of $\mathrm{Ti}^{3+}$ and $\mathrm{Ti}^{4+}$.

In figure 10.4 we plotted the average $3 d$ occupancy of $\mathrm{Ti}$ ions as the $\mathrm{Nb}$ content increases. The experimental profile fitting results deviated from the expected values based on the expected stoichiometric composition, indicating more $\mathrm{Ti}^{3+}$ character in the high $\mathrm{Nb}$ content sample and a composition deviation from the nominal stoichiometry. This deviation showed that the $\mathrm{Ti}$ ions received extra electrons that 
Table 10.1: The fractions of $\mathrm{Ti}^{3+}$ and $\mathrm{Ti}^{4+}$ components for each $\mathrm{BaTi}_{1-x} \mathrm{Nb}_{x} \mathrm{O}_{3}$ polycrystalline sample extracted by linear profile fitting to the reference spectra of $\mathrm{BaTiO}_{3}$ and $\mathrm{BaTi}_{0.5} \mathrm{Nb}_{0.5} \mathrm{O}_{3}$.

\begin{tabular}{llll}
\hline \multirow{2}{*}{ Sample } & \multicolumn{2}{c}{ Fraction } & \multirow{2}{*}{$\mathrm{R}^{2}$ value } \\
\cline { 2 - 3 } & \multicolumn{1}{c}{$\mathrm{Ti}^{4+}$} & \multicolumn{1}{c}{$\mathrm{Ti}^{3+}$} & \\
\hline $\mathrm{x}=0.004$ & $1.000(3)$ & $0.000(3)$ & 0.9994 \\
$\mathrm{x}=0.02$ & $1.000(20)$ & $0.000(20)$ & 0.9957 \\
$\mathrm{x}=0.10$ & $0.751(7)$ & $0.249(7)$ & 0.9995 \\
$\mathrm{x}=0.20$ & $0.464(12)$ & $0.537(12)$ & 0.9980 \\
\hline
\end{tabular}

were not originally from the $\mathrm{Nb}$-doping. Such extra electrons could come from the loss of oxygen ions and the subsequent release of electrons into the lattice. The loss of oxygen is sometimes inevitable, and has been observed in many oxide compounds. Although the donor-doping reduces the risk of loss oxygen ions, the low oxygen partial pressure used for synthesis samples on the other hand increased that risk. The compound can thus be described by a formula of $\mathrm{BaTi}_{1-x} \mathrm{Nb}_{x} \mathrm{O}_{3-\delta}$. For example, the most significant deviation for the $x=0.20$ sample can be induced by the loss of oxygen ions with $\delta=0.1$, which corresponds to $3.33 \%$ deviation from the oxygen stoichiometric composition. The loss of oxygen ions also found in $\mathrm{BaNbO}_{3-\delta}$ thin film (Gasparov et al., 2001) where $\delta=0.6-0.8$ depends on the growth conditions.

As a conclusion, energy loss near edge structure (ELNES) of the $\mathrm{Ti}^{-} \mathrm{L}_{2,3}$ of the mixed-valence $\mathrm{BaTi}_{1-x} \mathrm{Nb}_{x} \mathrm{O}_{3}$ compounds is investigated using high resolution electron energy loss spectroscopy (EELS) with an energy resolution of 0.23-0.27 $\mathrm{eV}$ and an energy dispersion of $0.03 \mathrm{eV} / \mathrm{Channel}$. After removing background and atomic cross section, each fraction of the $\mathrm{Ti}^{4+}$ and $\mathrm{Ti}^{3+}$ components is extracted 


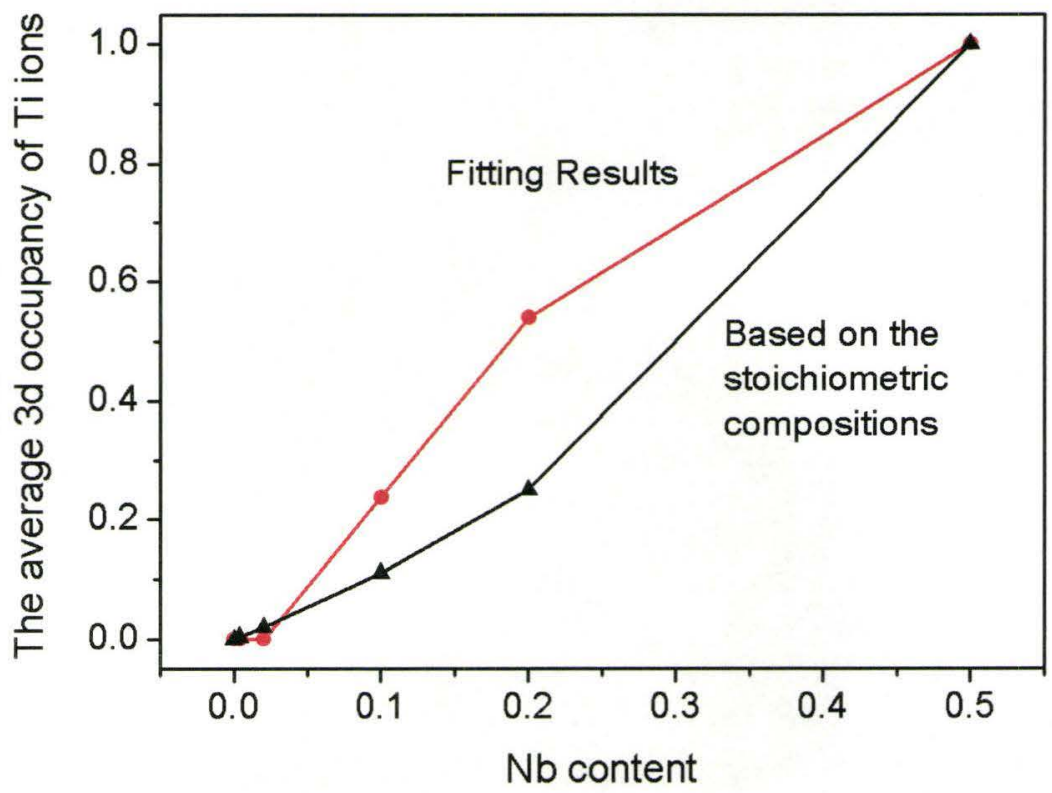

Figure 10.4: The average $3 d$ occupancy measured by profile fitting. The deviation of the profile fitting result as compare to the normal valence expected from the composition suggests a higher $\mathrm{Ti}^{3+}$ fractions in the compounds which could be from the loss of oxygen ions. Of note, is that the two points at $\mathrm{x}=0.002$ and $\mathrm{x}=0.5$ fitted so good are because we use them as the references.

by linear profile fitting of the corresponding $\mathrm{Ti}-\mathrm{L}_{2,3}$ edge to the $\mathrm{Ti}^{4+}$ and $\mathrm{Ti}^{3+}$ reference spectra obtained from $\mathrm{BaTi}_{0.998} \mathrm{Nb}_{0.002} \mathrm{O}_{3}$ and $\mathrm{BaTi}_{0.5} \mathrm{Nb}_{0.5} \mathrm{O}_{3}$ compounds which have the similar structures and chemical compositions to avoid possible peak broadening. The good fitting results indicate a higher $\mathrm{Ti}^{3+}$ fraction existing as $\mathrm{Nb}$ content increases, and suggest an oxygen deviation from its stoichiometry. A neutron diffraction result will be expected to confirm our above conclusion. 
PhD Thesis-Yang Shao-McMaster University-Materials Science and Engineering 2009 


\section{Chapter 11}

\section{Conclusion}

The continuous demand of miniaturization of devices by the micro-electronic industry boosts the developing of the thin films devices, especially for the promising ferroelectrics. Besides the optimum deposition conditions with suitable deposition methods, the bottom electrode materials, acting as the substrate at the same time, are also needed to be selected carefully. The ferroelectrics usually degenerate quickly with the traditional metal electrodes due to the oxygen vacancies in the films or around the interface. Hence the oxide electrodes are introduced to compensate the oxygen vacancies and improve the life time of the ferroelectrics. Although the oxide electrodes have lead to great improvement in term of the device life time, the performance of the ferroelectrics is also limited by the strain imposed by the widely used oxide electrodes, such as $\mathrm{IrO}_{2}, \mathrm{SrRuO}_{3}, \mathrm{LaNiO}_{3}$ and $\mathrm{La}_{0.5} \mathrm{Sr}_{0.5} \mathrm{CoO}_{3}$. Reducing the lattice mismatch between the oxide electrode and the ferroelectrics film is expected to further optimize the performance of the ferroelectrics. Recently, 
$\mathrm{Nb}$-doped $\mathrm{BaTiO}_{3}$ was found to be a suitable candidate bottom electrode with less lattice mismatch for the $\mathrm{Pb}\left(\mathrm{Zr}_{x} \mathrm{Ti}_{1-x}\right) \mathrm{O}_{3} / \mathrm{PbTiO}_{3}(\mathrm{PZT} / \mathrm{PT})$ ferroelectric films. In addition to being an excellent electrode material, $\mathrm{Nb}$-doped $\mathrm{BaTiO}_{3}$ also provides interesting electronic and magnetic properties that could be explored.

In my Ph.D. work, highly orientated $\mathrm{BaTi}_{1-x} \mathrm{Nb}_{x} \mathrm{O}_{3}$ thin films, spanning the entire range of $\mathrm{x}$, have been successfully deposited on (001) $\mathrm{MgAl}_{2} \mathrm{O}_{4}$ substrates by the pulsed laser deposition (PLD) method. X-ray diffraction measurements indicate single phase films for all values of $x$, contrary to a previously reported niobium solubility limit. Films show extreme sensitivity to high temperature oxygen exposure, which destroys conductivity and severely disrupts crystallinity. Under optimum growth conditions increasing $x$ gives rise to a valence change from $\mathrm{Ti}^{4+}$ to $\mathrm{Ti}^{3+}$ transformation in the oxidation state accompanied by increased conductivity with a semiconductor-metal transition near $x=0.2$. Temperature dependent magnetic measurements show an anomalous rise in the spin moment, a feature supportive of the small singlet bipolaron model or the lifting of the electron degeneracy of the conduction band in the low-symmetry phases of the $\mathrm{BaTi}_{1-x} \mathrm{Nb}_{x} \mathrm{O}_{3}$. Among the $\mathrm{BaTi}_{1-x} \mathrm{Nb}_{x} \mathrm{O}_{3}$ thin films, the $x=0.5$ film is the best one for use as the bottom electrode due to the high conductivity in spite of a $1 \%$ lattice mismatch remaining.

A partial strontium-for-barium substitution was used in this work to further reduce the lattice mismatch and keep the conductivity at the same time. With this aim, epitaxial (001) oriented films of the metallic oxide $\left(\mathrm{Ba}_{1-y} \mathrm{Sr}_{y}\right) \mathrm{Ti}_{0.5} \mathrm{Nb}_{0.5} \mathrm{O}_{3}$, with $y=0,0.4,0.5$ and 0.6 , were deposited onto (001) $\mathrm{MgAl}_{2} \mathrm{O}_{4}$ substrates. The strontium for barium substitution gives rise to a decrease in the lattice constant, 
without altering the conductivity of the film. In a more general sense, such a substitution provides a means for independently tuning the lattice parameter and conductivity over a significant range of values. X-ray diffraction measurements show a significant number of [111], [011] and [221] misoriented grains, that are not present for the $y=0.6$ composition. Transmission electron microscopy images of the filmsubstrate interface obtained for the $y=0.6$ composition show a sharp interface with flawless epitaxy. We attribute the improvements to a decrease in the lattice misfit strain made possible through the superior lattice match to the substrate obtained through strontium substitution.

In addition to the application as the bottom electrode materials, $\mathrm{BaTi}_{0.5} \mathrm{Nb}_{0.5} \mathrm{O}_{3}$ is also a sub-class of transition metal oxide materials that has demonstrated a wide variety of interesting electrical and magnetic properties. Since the electronic structures play the crucial role in determining the physical properties of the transition metal oxides, we explored in the electronic structure evolution and the valence change as the $\mathrm{Nb}$ ions substitution. The electronic structure calculations were carried out by the $1^{s t}$ principle method using the WIEN2k program, which is based on the full-potential (linearized) augmented plane wave (LAPW) plus local orbitals (lo) methods under the frame work of the density functional theory (DFT). Based on the assumed ordered structures, my calculations of the density of states for $\mathrm{BaTi}_{1-x} \mathrm{Nb}_{x} \mathrm{O}_{3}(x=0.125,0.25,0.5,0.75$ and 1$)$ showed that the Fermi level gradually moved to the lower energies, consistent with the increase of the conduction electron density with $\mathrm{Nb}$ content as measured from the Hall effect. The valence bands were not significantly altered with the $\mathrm{Nb}$ ions substitutions. 
The electronic structure of the series of mixed-valence $\mathrm{BaTi}_{1-x} \mathrm{Nb}_{x} \mathrm{O}_{3}$ compounds was also investigated experimentally using high resolution electron energy loss spectroscopy (EELS). The energy loss near edge structure (ELNES) of the Ti$L_{2,3}$ edge and $\mathrm{O}-\mathrm{K}$ were recorded with an energy resolution of $0.24-0.27 \mathrm{eV}$. The fraction of each $\mathrm{Ti}^{4+}$ and $\mathrm{Ti}^{3+}$ component in samples was extracted by fitting the shape of the corresponding $\mathrm{Ti}-L_{2,3}$ edge to the $\mathrm{Ti}^{4+}$ and $\mathrm{Ti}^{3+}$ reference spectra obtained from $\mathrm{BaTi}_{0.998} \mathrm{Nb}_{0.002} \mathrm{O}_{3}$ and $\mathrm{BaTi}_{0.5} \mathrm{Nb}_{0.5} \mathrm{O}_{3}$ compounds. The good fitting results indicate higher $\mathrm{Ti}^{3+}$ fractions than excepted in the $\mathrm{BaTi}_{1-x} \mathrm{Nb}_{x} \mathrm{O}_{3}$ as $\mathrm{Nb}$ content increase, which could be the result from the loss of oxygen stoichiometry. This new methods used here might be used to other mixed-valence systems or probe local charges in valence. A crystal field parameter of $2.10 \mathrm{eV}$ was also extracted from the Ti- $L_{2,3}$ edge for the pseudocubic $\mathrm{BaTiO}_{3}$. The $\mathrm{O}-K$ edge was analyzed by compared to the partial density of states calculation. The detailed feature evolutions of O-K edge are explained by the decrease of the Ti $3 d$ band contribution and increase of the $\mathrm{Nb} 4 d$ band contribution. A $1.2 \mathrm{eV}$ chemical shift is also found from $\mathrm{BaTiO}_{3}$ to $\mathrm{BaNbO}_{3}$.

Overall, comparison of experiment and theory is extremely useful in understanding very interesting electronic structure effects and good material properties. 


\section{Appendix A}

\section{Grain Orientation of $\mathrm{BaTi}_{1-x} \mathrm{Nb}_{x} \mathrm{O}_{3}$}

\section{Thin Films}

Grain orientations of thin films were studied with the help of pole figures. The single crystal X-ray diffraction data are collected by a three-circle Bruker D8 diffractometer with a 2D SMART6000 CCD detector. After processing the raw data, the desired pole figures can be generated by the General Area Detector Diffraction System (GADDS). The geometry of the film mounted on the goniometer stage is illustrated in figure A.1. All of the generated pole figures are projected to the $z$ direction which is normal to the surface of the films. Two selected conducting films (see section 7.8 on page 121 ) with $x=0.50$ and $x=1.0$ on (001) $\mathrm{MgAl}_{2} \mathrm{O}_{4}$ are examined, and films' $\{001\},\{011\}$ and $\{111\}$ pole figures are generated for each sample. To avoid complexity introduced by other reflections, one integration range 

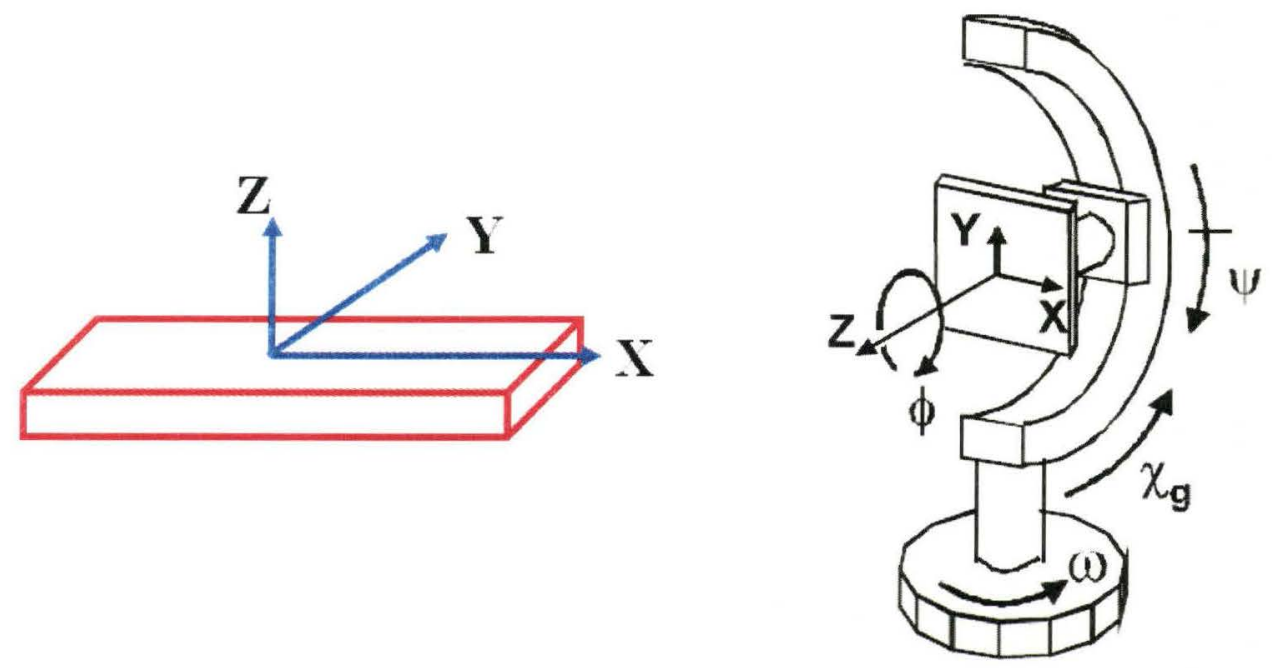

Figure A.1: The coordination relationship of the film and 2D XRD sample state

as narrow as possible was used in the experiments (see table A.1) and was applied to each generated pole figure. Of course, each pole figure will contain both orientation information from the films and the substrate as indicated in table A.1.

Table A.1: Integration range for each pole figure

\begin{tabular}{ccl}
\hline Pole figure & Integration range $(2 \theta)$ & Covered reflections \\
\hline$\{001\}$ & $42.3-46.7^{\circ}$ & substrate $(004)+$ film $(002)$ \\
$\{011\}$ & $30.2-32.0^{\circ}$ & substrate $(022)+$ film $(011)$ \\
$\{111\}$ & $36.0-38.0^{\circ}$ & substrate $(311)+$ film $(111)$ \\
\hline
\end{tabular}

Since our pseudo-cubic films are highly [001] orientated on (001) cubic substrate, we can have a reasonable guess before doing experiments that it will be difficult for us to subtract substrate information from films' $\{001\}$ and $\{011\}$ pole figures, but films' other grain orientations are prominent due to the relatively high structure factor for (001) and (011) reflections. Instead, the films' $\{111\}$ pole figure can be well separated from substrate and films reflection as one can see from table 


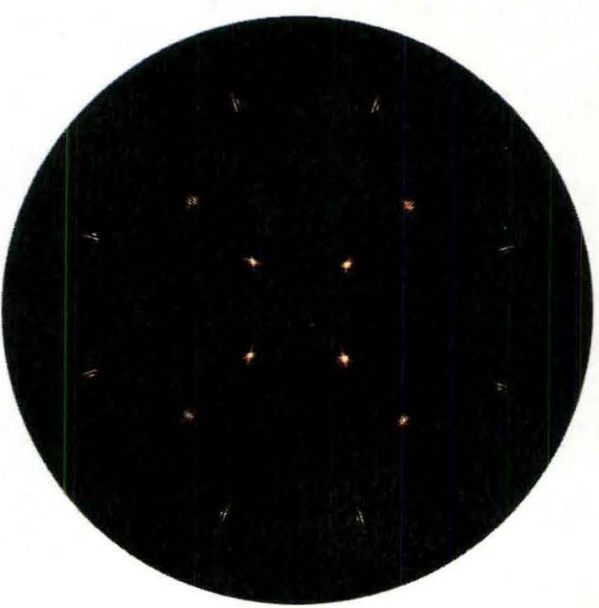

(a) $\mathrm{BaTi}_{1-x} \mathrm{Nb}_{x} \mathrm{O}_{3}$ thin film at $\mathrm{x}=0.50$

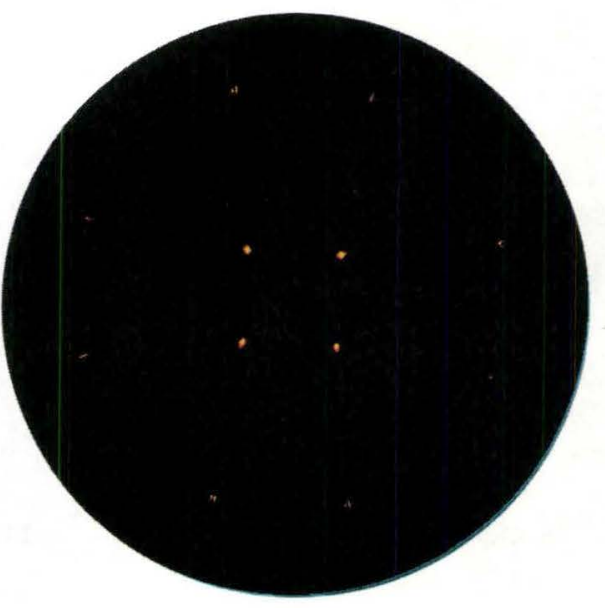

(c) $\mathrm{MgAl}_{2} \mathrm{O}_{4}$ substrate only

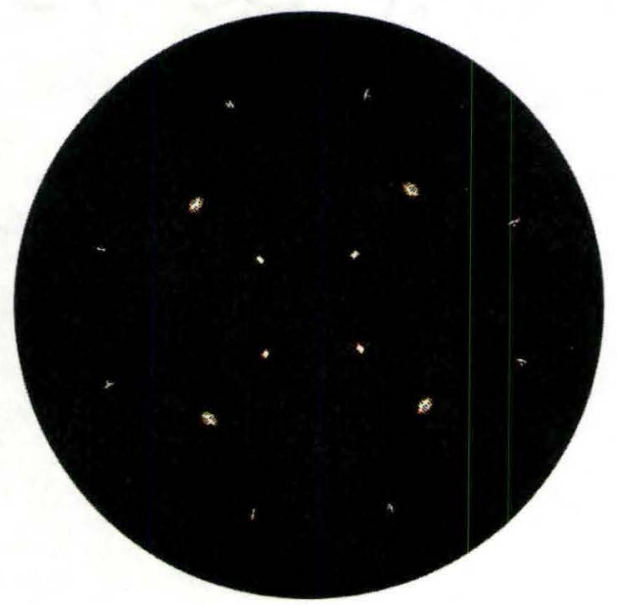

(b) $\mathrm{BaTi}_{1-x} \mathrm{Nb}_{x} \mathrm{O}_{3}$ thin film at $\mathrm{x}=1.0$

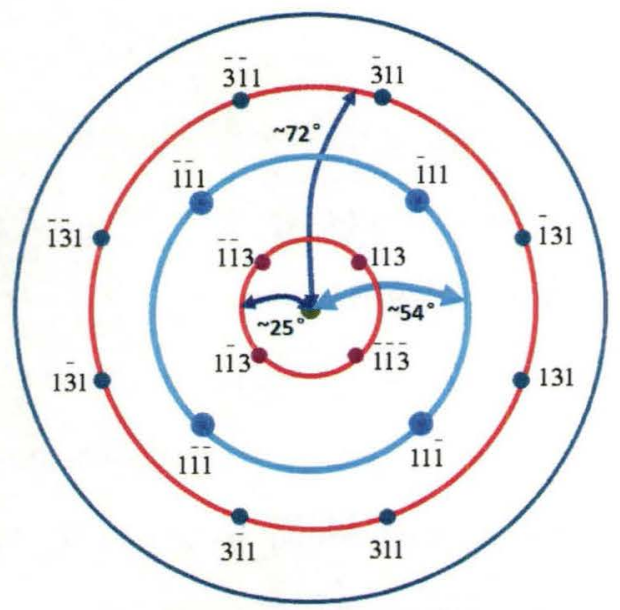

(d) figure of $\{311\}$ and $\{111\}$ poles

Figure A.2: Pole figures generated from $2 \theta$ range of $36.0-38.0^{\circ}$ demonstrates a combination figure of substrate $\{311\}$ poles and film's $\{111\}$ poles for (a) $\mathrm{BaTi}_{1-x} \mathrm{Nb}_{x} \mathrm{O}_{3}$ thin film at $\mathrm{x}=0.50$ composition, and (b) $\mathrm{BaTi}_{1-x} \mathrm{Nb}_{x} \mathrm{O}_{3}$ thin film at $\mathrm{x}=1.0$ composition. (c) shows a pure $\{311\}$ pole figure of the $\mathrm{MgAl}_{2} \mathrm{O}_{4}$ substrate only. (d) presents an indexed combinational pole figure for one to understand the experimental results

A.1, however, other grain orientations are weak due to the lower structure factor. Therefore we can study the films' main orientation relationship to the substrate by $\{111\}$ pole figure and other grain orientations by $\{001\}$ and $\{011\}$ pole figures. 
The $\{111\}$ pole figures of $\mathrm{BaTi}_{1-x} \mathrm{Nb}_{x} \mathrm{O}_{3}$ thin films at $x=0.50$ and $x=1.0$ composition are generated in the $2 \theta$ range of $36.0-38.0^{\circ}$ as shown in figure A.2(a) and A.2(b). They are a combination of substrate's (311) and film (111) poles as indicated in table A.1. A pure $\mathrm{MgAl}_{2} \mathrm{O}_{4}$ substrate's $\{311\}$ pole figure is illustrated in figure A.2(c) with 12 sharp spots, and a combined figure of (311) poles and (111) poles is also established in figure A.2(d), which is presented to help understand figures A.2(a) and A.2(b) more easily. Comparing to the figure A.2(c) and A.2(d), from figure A.2(a)and A.2(b), one can easily identify the highly symmetric 12 sharp (311) poles of the substrate, and 4 strong (111) poles of the films. As we have shown that all our films are highly [001] orientated from $\theta-2 \theta$ XRD data, we still lack of the in-plane grain orientation information. The presence of the 4 (111) poles on the stereoprojection plane without any splitting indicate that the main orientation of the $x=0.50$ and $x=1.0 \mathrm{BaTi}_{1-x} \mathrm{Nb}_{x} \mathrm{O}_{3}$ films. There is no in-plane rotation for the main grain orientation. Since the $\mathrm{BaTi}_{1-x} \mathrm{Nb}_{x} \mathrm{O}_{3}$ thin films are deposited and cooled in the same condition, we can expect they are all epitaxial not only in the surface normal direction but also in the in-plane direction, at least for $x=0.50$ and $x=1.0$ composition.

The $\{001\}$ pole figures of $\mathrm{BaTi}_{1-x} \mathrm{Nb}_{x} \mathrm{O}_{3}$ thin films at $x=0.50$ and $x=1.0$ composition are generated in the $2 \theta$ range of $42.3-46.7^{\circ}$ as shown in figure A.3(a) and A.3(b). In this $2 \theta$ range, only the substrate (004) and the films (002) pole are recorded as mentioned in table A.1. There is only one major spot at the center of each pole figure with twenty-four minor spots symmetrically dispersed around it. Since the substrate is a commercial single crystal, its (004) pole would not contribute to any other spots except the central one in figure A.3(a) and A.3(b).The 


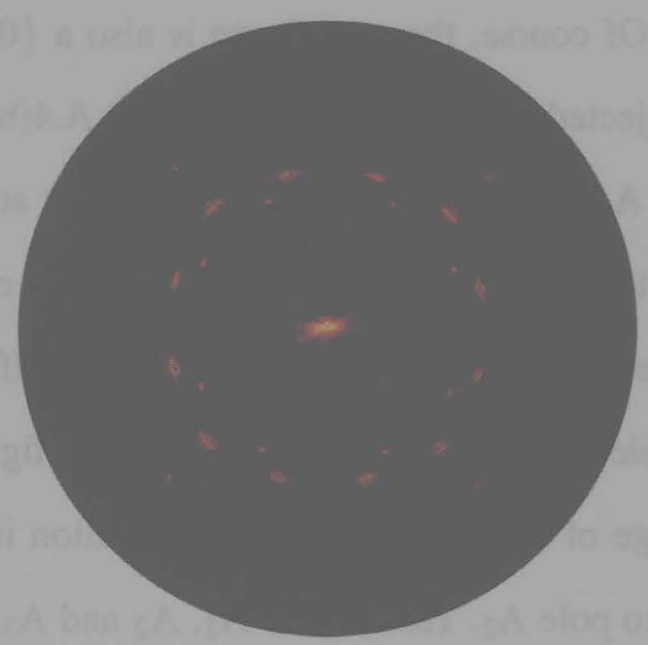

(a) $\mathrm{BaTi}_{1-x} \mathrm{Nb}_{x} \mathrm{O}_{3}$ thin film at $\mathrm{x}=0.50$

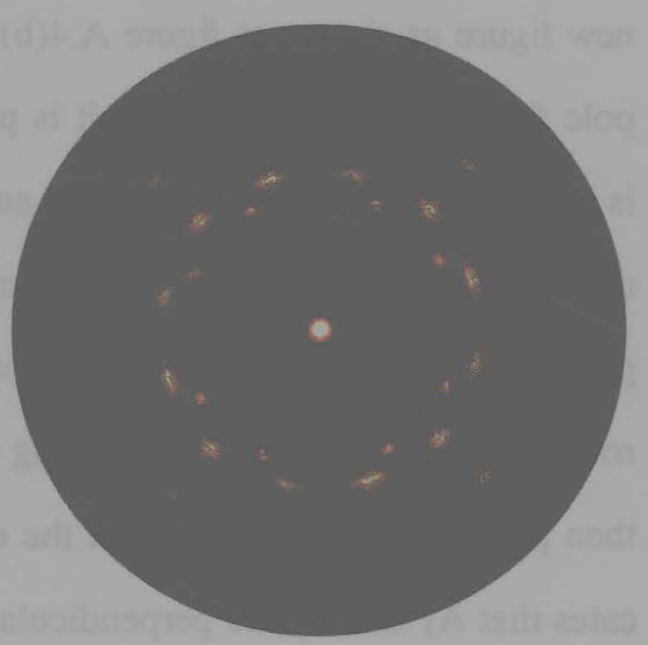

(b) $\mathrm{BaTi}_{1-x} \mathrm{Nb}_{x} \mathrm{O}_{3}$ thin film at $\mathrm{x}=1.0$

Figure A.3: Films' $\{001\}$ pole figures for $\mathrm{BaTi}_{1-x} \mathrm{Nb}_{x} \mathrm{O}_{3}$ thin film at $\mathrm{x}=0.5$ and 1.0, generated from $2 \theta$ range of $42.3-46.7^{\circ}$ (a) $\mathrm{BaTi}_{1-x} \mathrm{Nb}_{x} \mathrm{O}_{3}$ thin film at $\mathrm{x}=0.50$ composition, and (b) $\mathrm{BaTi}_{1-x} \mathrm{Nb}_{x} \mathrm{O}_{3}$ thin film at $\mathrm{x}=1.0$ composition.

(002) pole of films' main orientation, however, will also show in the center of stereoprojection plane but merges into the strongly excited (004) pole of the substrate and therefore can not be distinguished. We assign it as the first set of orientation in our film. Except the bright central spot, other spots on figure A.3(a) and A.3(b) are related to the $(001)$ poles from the films. Their reflections will provide more information about the minor grain orientations.

To analyze other poles, we classify all other 24 non-central poles into two groups: group A on the band indicated in figure A.4(a) and group B outside the band. For each group, we simply pick up three poles and mark them $\operatorname{as}\left(\mathrm{A}_{1}, \mathrm{~A}_{2}\right.$, and $\left.A_{3}\right)$ and $\left(B_{1}, B_{2}\right.$, and $\left.B_{3}\right)$ (figure $A .4(a)$ ), as an example to expatiate how they relate to two orientations. To explain the relationship between poles $A_{1}, A_{2}$, and $A_{3}$, we rotated the pole figure to a given angle, letting pole $A_{1}$ at the center of the 
new figure as shown as figure A.4(b). Of course, the new figure is also a $\{001\}$ pole figure of the thin film but it is projected from pole $A_{1}$. On figure A.4(b), it is found that the previous poles $A_{2}$ and $A_{3}$ in figure A.4(a) now are present at the edge of the new pole figure. According to the stereoprojection, it is clear that each pole $A_{2}$ and $A_{3}$ is perpendicular to pole $A_{1}$ in the new pole figure $A .4(b)$. If we rotate the pole figure again by letting pole $A_{2}$ at the center of the new pole figure, then poles $A_{1}$ and $A_{3}$ will be at the edge of new pole figure. This rotation indicates that $A_{1}$ and $A_{3}$ are perpendicular to pole $A_{2}$. Hence pole $A_{1}, A_{2}$ and $A_{3}$ are normal to each other. Together with the determination of the cubic structure from our previous XRD analysis (see section 7.2 on page 94), the three pole $A_{1}, A_{2}$ and $A_{3}$ represent only one orientation but different lattice axis (namely [200], [020] and [002]), and here we call it as the second set of orientation. Applying the same method, the third set of orientation represented by pole $B_{1}, B_{2}$ and $B_{3}$ can be found and their relationships are shown in figure A.4(c). If we analyse all the twenty-four poles, except the central one shown in figure A.4(a), eight possible minor orientations can be found: four belong to the second set of orientation and four belong to the third set of orientation. For the second and third set of orientations, the film surface normal are $<111>$ and $<221>$ direction respectively, which is consistent with the weak (111) peaks found in $\theta-2 \theta$ XRD spectra for $x=0.50$ and $x=1.0$ composition. The weak (011) reflection shown in the XRD data for $x=0.50$ and $x=1.0$ suggest a ( 011$)$ orientated grain. However, due to its lower intensity, these orientation is barely detectable for $x=0.50$ and difficult for $x=1.0$. The $<221>$ reflection showed on figure 7.2 around $69^{\circ}$ and might overlap with (003) reflections for our pseudo-cubic structure because the sum of the $h^{2}+k^{2}+l^{2}$ is the same for both (221) and (003). The $<221>$ orientated grain could be due to forming twin 
structures with the main orientation (see section 7.4 on page 102).

With the main orientation, there are totally nine possible orientations in our films at $x=0.50$ and $x=1.0$ composition. Each of the nine possible different orientations was shown in figure A.4(d), represented by the same symbol and connected by lines. The first set of orientation is represented by a big solid circle at the center, the four second set of orientations $(<111>$ orientated grains) are represented by the same solid symbol and connected by the solid line, and the four third set of orientation ( $<221>$ orientated grains) are represented by the same open symbol and connected by the dash line. The nine possible orientations could arise from lattice misfit between substrate and films or non-optimized deposition condition. Such highly symmetric $\{001\}$ pole figure suggests there may exist twin structure.

The $\{011\}$ pole figures of $\mathrm{BaTi}_{1-x} \mathrm{Nb}_{x} \mathrm{O}_{3}$ thin films at $x=0.50$ and $x=1.0$ composition are also generated in the $2 \theta$ range of $30.2-32.0^{\circ}$ as shown in figure A.5(a) and A.5(b). There are four strong poles sitting on the single crystal (011) pole positions and twenty-four other poles symmetrically around. Such pattern of the pole figure is because of more orientations in the films which have been clarified in in previous $\{001\}$ pole figure (figure A.4). In the $\{011\}$ pole figures, the (011) poles of films' main orientation are also overlapping with substrate $(011)$ poles which are shown in the figure A.5(c). To illustrate the relationship between other poles is more difficult but the basic idea is the same as what we have explained in the $\{001\}$ pole figure (figure A.4). The difference is that (011) poles are not normal to each other and we have to use Wulff net as an assistance. Since $\{001\}$ pole figure is 


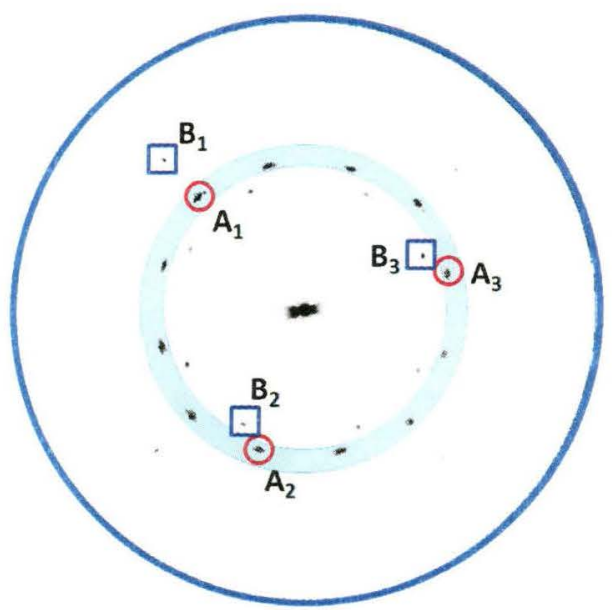

(a)

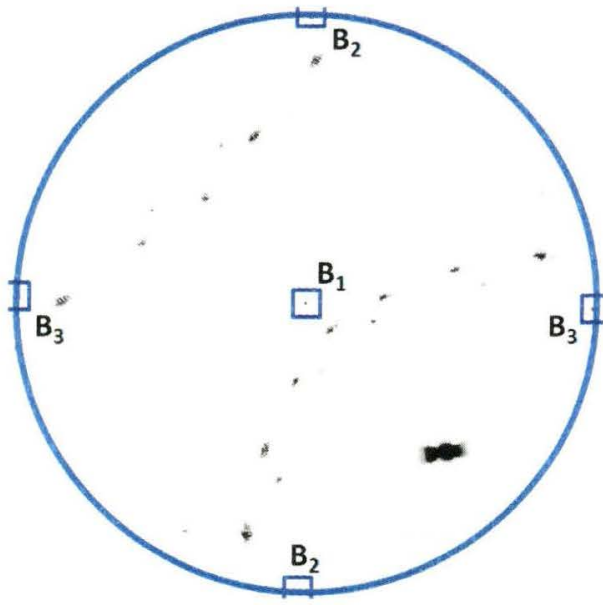

(c)

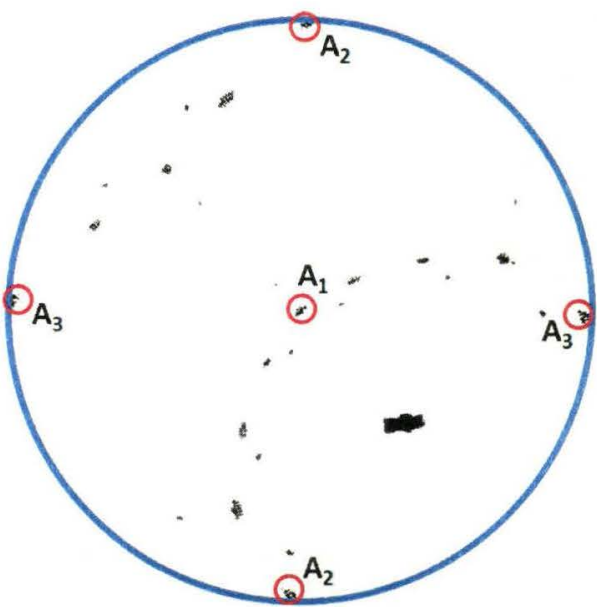

(b)

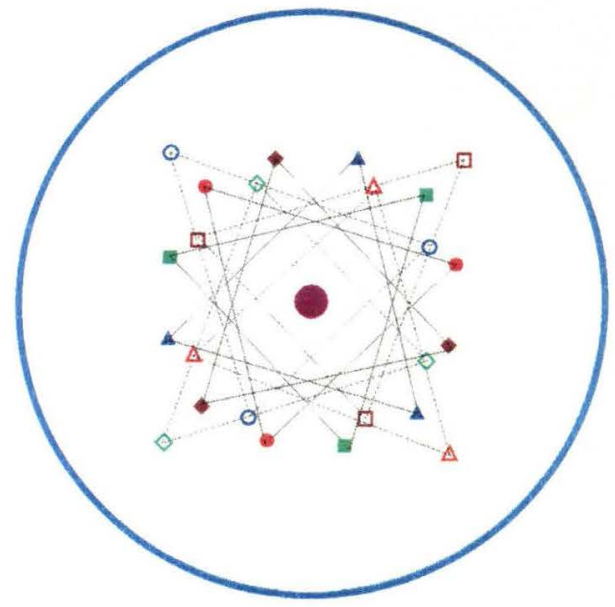

(d)

Figure A.4: Nine possible grain orientations exist in $\mathrm{BaTi}_{1-x} \mathrm{Nb}_{x} \mathrm{O}_{3}$ thin film at $x=0.5$ and $x=1.0$. by $\{001\}$ pole figures (a) twenty-four non-central poles categorize into group $A$ and $B$. For each group, three poles $\left(A_{1}, A_{2}\right.$ and $\left.A_{3}\right)\left(B_{1}, B_{2}\right.$ and $\left.B_{3}\right)$ are selected as an example to illustrate their relationship (b) A new pole figure generated by rotating the original pole figure (a) a certain angle to let pole $A_{1}$ at the center. After rotating, pole $A_{2}$ and $\mathrm{A}_{3}$ are right at the edge of the new pole figure. (c) An other new pole figure generated by rotating the original pole figure (a) a certain angle to let pole $\mathrm{B}_{1}$ at the center. After rotating, pole $B_{2}$ and $B_{3}$ are right at the edge of the new pole figure. (d) A possible nine-orientation model presented to explain the $\{001\}$ pole figure. Each orientation is represented by the same symbol and connected by the corresponding lines. 
more easy to understand the grain orientations and there is no more information we can get from $\{011\}$ pole figure. Here we only present the final result in the figure A.5(d)). Of course, nine grain orientation are found. The same orientations are marked with same symbols and connected with lines. Moreover, for convenience, the same grain orientation in the $\{001\}$ (figure A.4(d)) and $\{011\}$ (figure A.5(d)) pole figures is marked by the same type of symbols. It is clearly to see that the third set orientations ( $<221>$ orientated grains) as described in figure A.4 by the same open symbol and connected by the dash line, share two $<011>$ directions with the main orientation and form twin structure (see section 7.4 on page 102). 


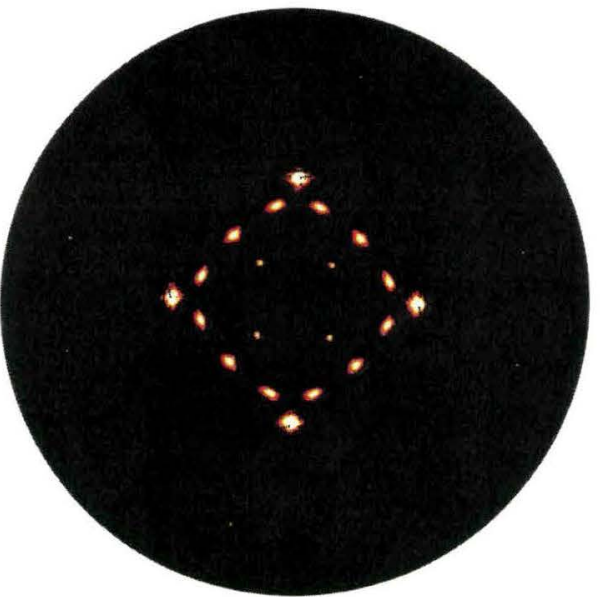

(a)

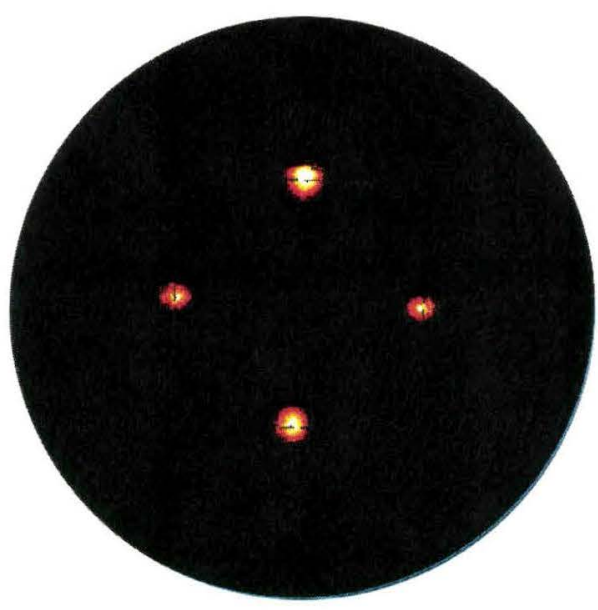

(c)

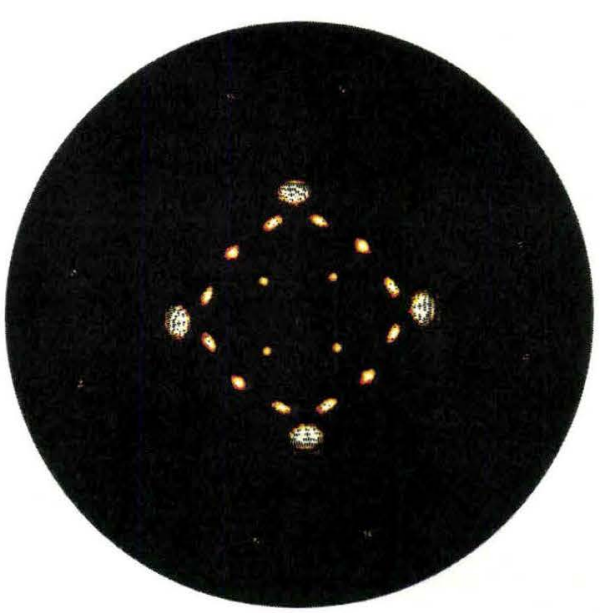

(b)

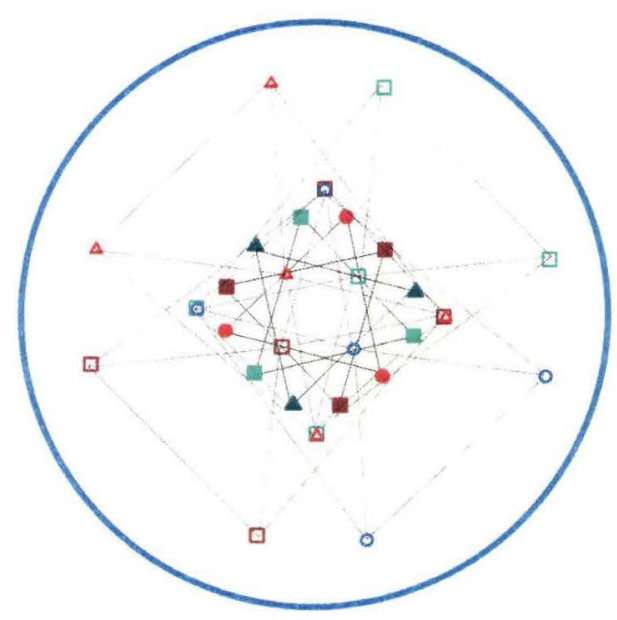

(d)

Figure A.5: Films' $\{011\}$ pole figures for $\mathrm{BaTi}_{1-x} \mathrm{Nb}_{x} \mathrm{O}_{3}$ thin film at $x=0.5$ and 1.0 composition, generated from $2 \theta$ range of $30.2-32.0^{\circ}$. Nine possible grain orientations are confirmed. (a) $\mathrm{BaTi}_{1-x} \mathrm{Nb}_{x} \mathrm{O}_{3}$ thin film at $\mathrm{x}=0.50$ composition (b) $\mathrm{BaTi}_{1-x} \mathrm{Nb}_{x} \mathrm{O}_{3}$ thin film at $\mathrm{x}=1.0$ composition (c) a pure $\{011\}$ pole figure of the $\mathrm{MgAl}_{2} \mathrm{O}_{4}$ substrate only (d) Nine possible grain orientations are confirmed. Each orientation is represented by the same symbol and connected by the corresponding lines. 


\section{Appendix B}

\section{Pole Figures of}

\section{$\left(\mathrm{Ba}_{1-y} \mathrm{Sr}_{y}\right) \mathrm{Ti}_{0.5} \mathrm{Nb}_{0.5} \mathrm{O}_{3}$ Thin Films}

Additional structural information of $\left(\mathrm{Ba}_{1-y} \mathrm{Sr}_{y}\right) \mathrm{Ti}_{0.5} \mathrm{Nb}_{0.5} \mathrm{O}_{3}$ thin films was obtained using 2D-XRD techniques. The data was collected by a three-circle Bruker D8 diffractometer with a 2D SMART6000 CCD detector. The $2 \theta$ integration windows used in the generation of various pole figures are listed in table 9.2. All $\{001\}$ and $\{011\}$ pole figures of $\left(\mathrm{Ba}_{1-y} \mathrm{Sr}_{y}\right) \mathrm{Ti}_{0.5} \mathrm{Nb}_{0.5} \mathrm{O}_{3}$ thin films with all values of $y$ are shown in the figure B.1 and B.2, respectively. 


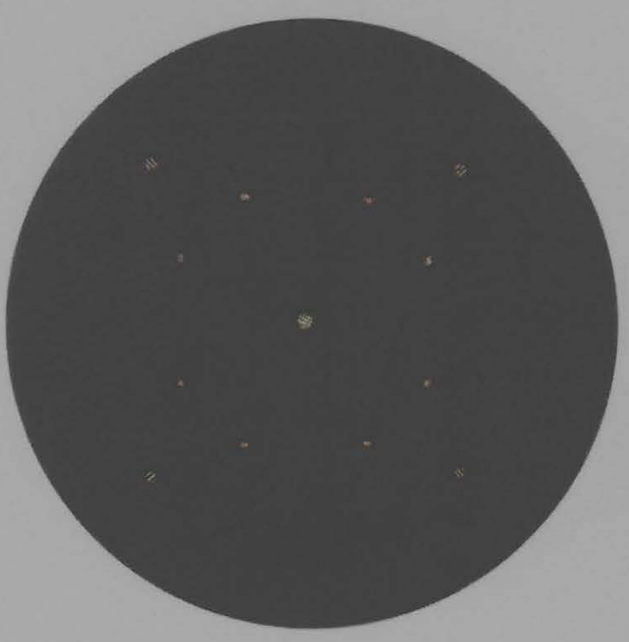

(a) $\mathrm{y}=0$

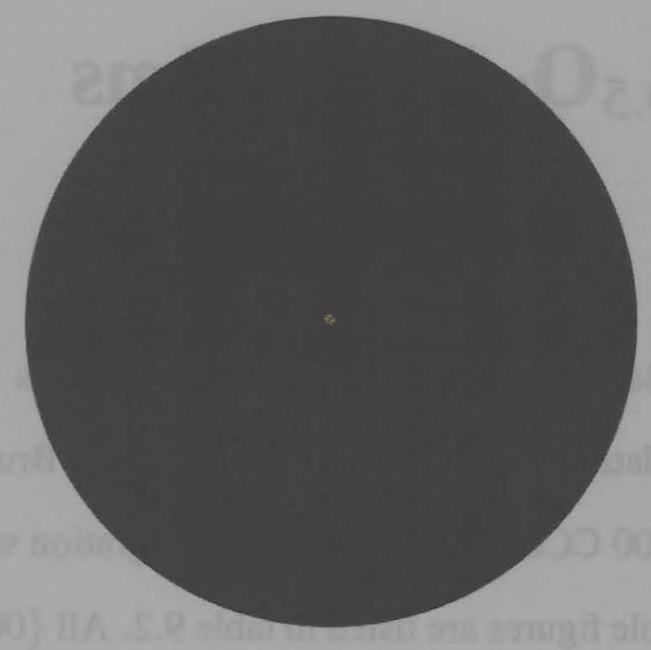

(c) $\mathrm{y}=0.50$

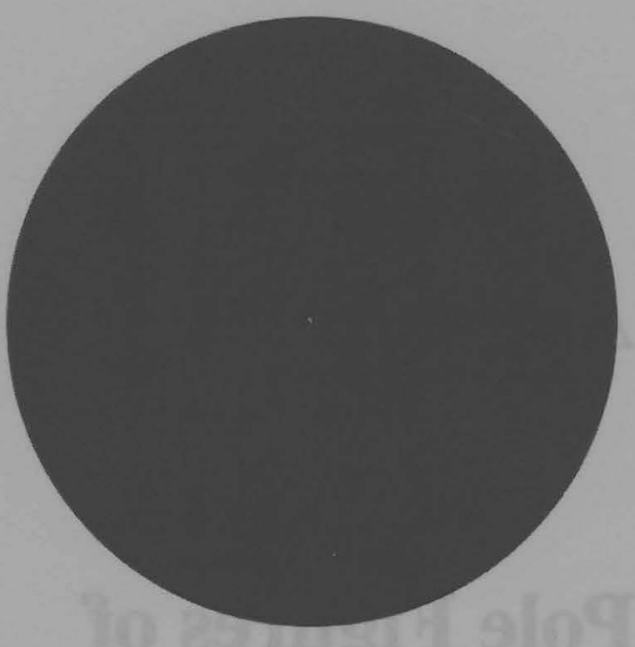

(b) $\mathrm{y}=0.40$

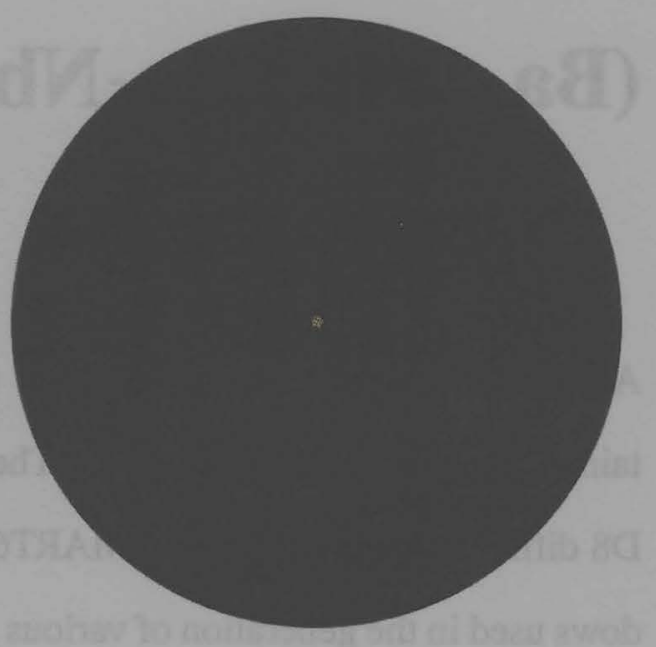

(d) $\mathrm{y}=0.60$

Figure B.1: (001) pole figure of $\left(\mathrm{Ba}_{1-y} \mathrm{Sr}_{y}\right) \mathrm{Ti}_{0.5} \mathrm{Nb}_{0.5} \mathrm{O}_{3}$ thin films for (a) $\mathrm{y}=0$, (b) $\mathrm{y}=0.40$, (c) $y=0.50$ and (d) $y=0.60$. There are four miner (221) orientated grains plus one major (001) orientated grins for $\mathrm{y}=0$ film, and only one major (001) orientated grains for $\mathrm{y}=0.40$, 0.50 and 0.60 films. 


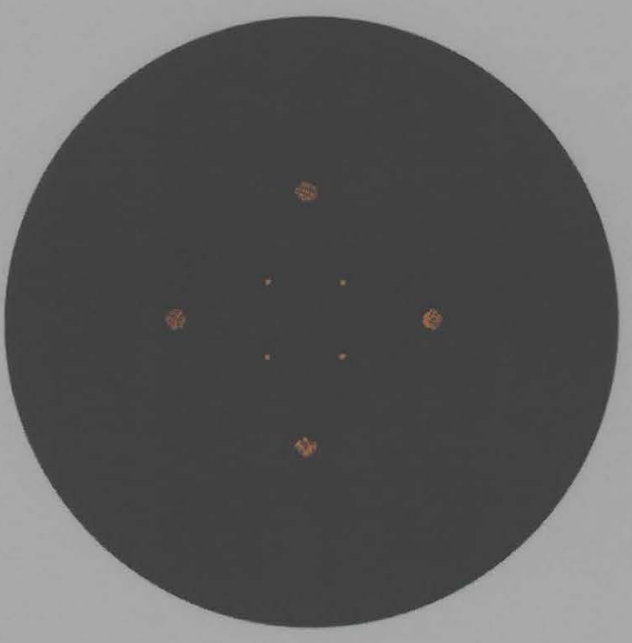

(a) $\mathrm{y}=0$

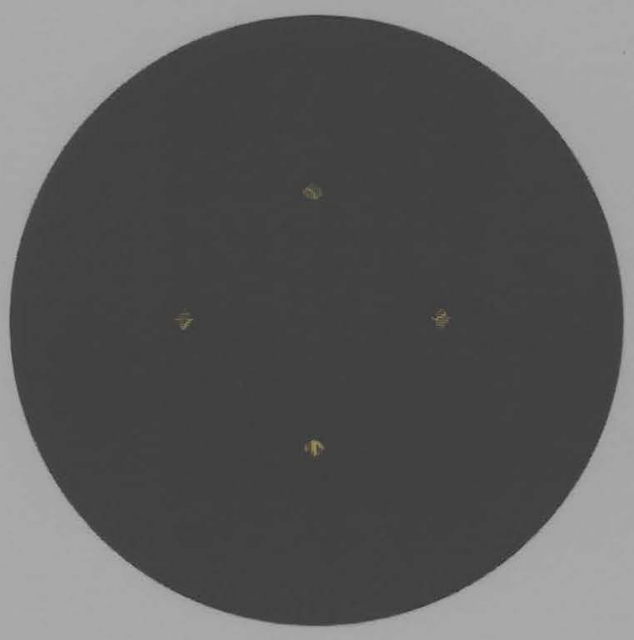

(c) $\mathrm{y}=0.50$

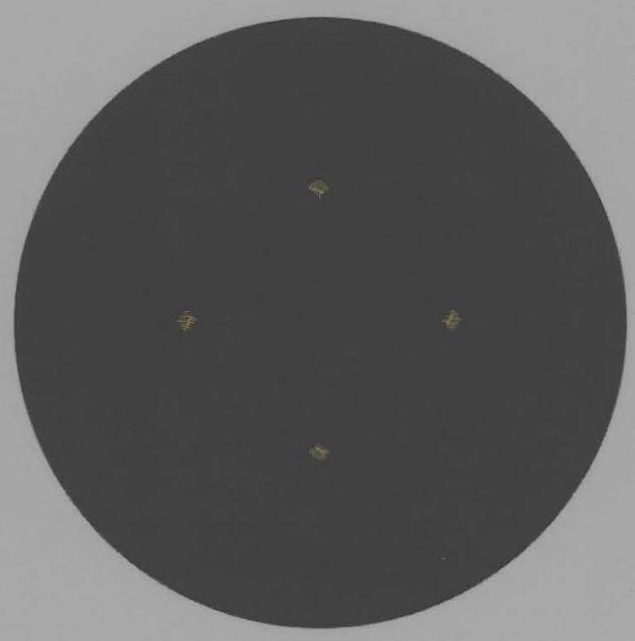

(b) $\mathrm{y}=0.40$

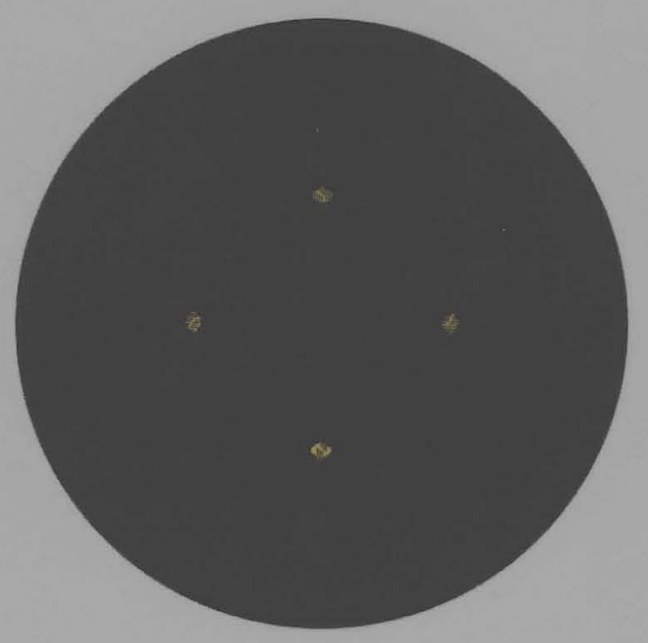

(d) $\mathrm{y}=0.60$

Figure B.2: (011) pole figure of $\left(\mathrm{Ba}_{1-y} \mathrm{Sr}_{y}\right) \mathrm{Ti}_{0.5} \mathrm{Nb}_{0.5} \mathrm{O}_{3}$ thin films for (a) $\mathrm{y}=0$, (b) $\mathrm{y}=0.40$, (c) $y=0.50$ and (d) $y=0.60$ There are four miner (221) orientated grains plus one major (001) orientated grins for $\mathrm{y}=0$ film, and only one major $(001)$ orientated grains for $y=0.40,0.50$ and 0.60 films. 
PhD Thesis-Yang Shao-McMaster University-Materials Science and Engineering 2009 


\title{
Appendix C
}

\section{Lorentzian Broadening Program}

\author{
\%\%\%\%\%\%\%\%\%\%\%\%\%\%\%\%\%\%\%\%\%\%\%\%\%\%\%\%\%\%\%\% \\ $\% \% \% \quad$ Lorentzian broadening program \\ $\% \% \% \quad$ Matlab Script \\ $\% \% \% \quad$ by Yang Shao, Nov 2008 \\ \%\%\%\%\%\%\%\%\%\%\%\%\%\%\%\%\%\%\%\%\%\%\%\%\%\%\%\%\%\%\%\% \\ clear; \% Clear variables \\ clc; \% Clear screan
}

\% Import data from file 'EELSSpectra.dat'

\% The 'EELSSpectra.dat' file should contain two column:

$\%$ one is for Energy, the other one is for Intensity.

file 1 = 'EELSSpectra.dat';

[c1 c2] = textread(file1, '\%f \%f', 1000, 'headerlines',0, 'commentstyle', 'shell'); 


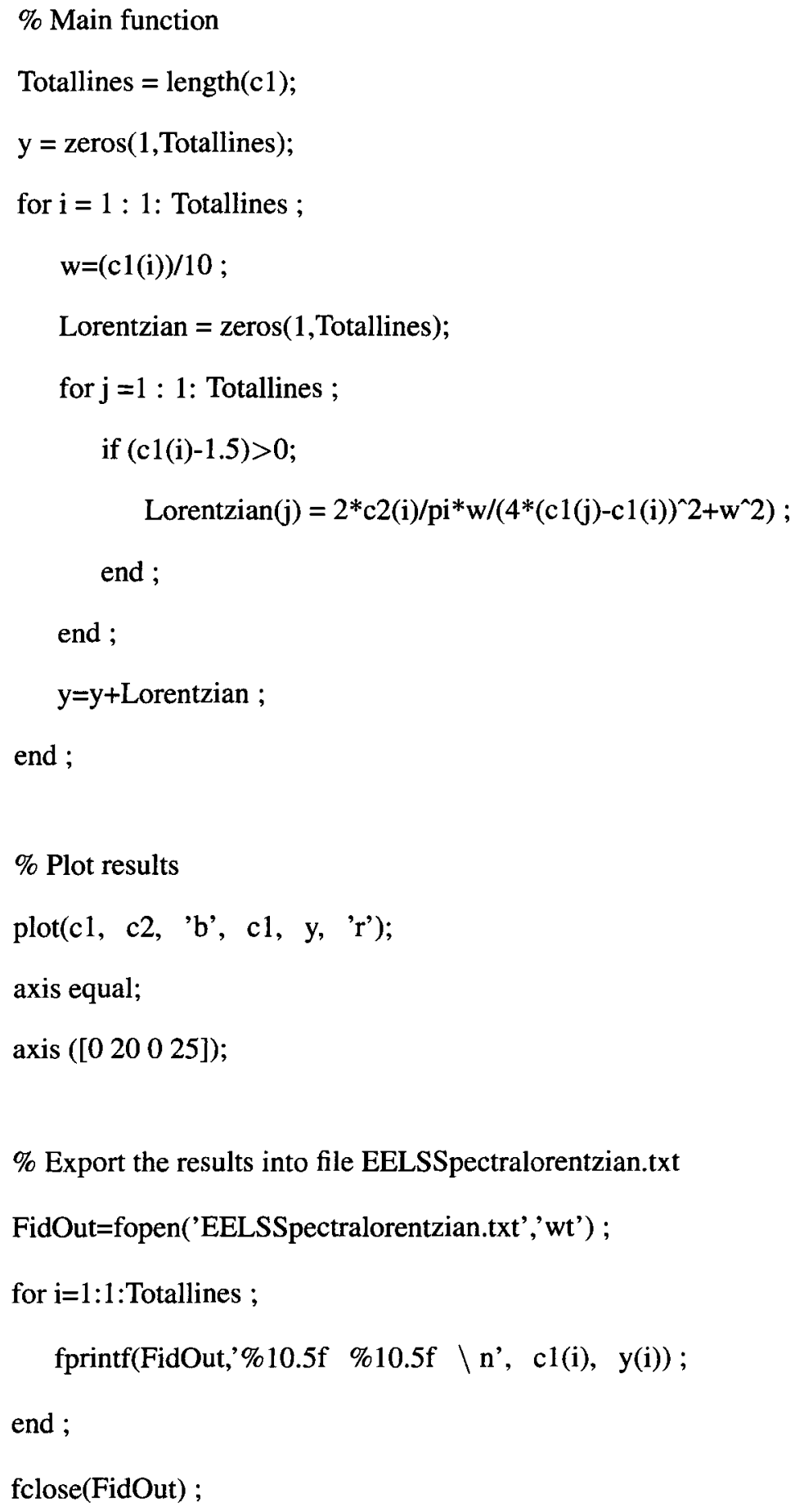




\section{References}

Abbate, M., de Groot, F. M. F., Fuggle, J. C., Fujimori, A., Tokura, Y., Fujishima, Y., Strebel, O., Domke, M., Kaindl, G., Vanelp, J., Thole, B. T., Sawatzky, G. A., Sacchi, M., \& Tsuda, N. 1991. Soft-X-ray-absorption studies of the location of extra charges induced by substitution in controlled-valence materials. Physical Review B, 44(11), 5419-5422.

Anderson, M. T., Greenwood, K. B., Taylor, G. A., \& Poeppelmeier, K. R. 1993. B-Cation arrangements in double perovskites. Progress in Solid State Chemistry, 22(3), 197233.

Anderson, P. W. 1958. Absence of diffusion in certain random lattices. Physical Review, $109(5), 1492$.

Andrews, P., Fuggle, J. C., \& Inglesfield, J. E. 1992. Unoccupied electronic states : fundamentals for XANES, EELS, IPS and BIS. Berlin ; New York : Springer-Verlag.

Arya, P. R., Jha, P., \& Ganguli, A. K. 2003. Synthesis, characterization and dielectric properties of nanometersized barium strontium titanates prepared by the polymeric citrate precursor method. Journal of Materials Chemistry, 13(2), 415-423.

Bi, C. Z., Ma, J. Y., Yan, J., Fang, X., Zhao, B. R., Yao, D. Z., \& Qiu, X. G. 2006. Electronphonon coupling in $\mathrm{Nb}$-doped $\mathrm{SrTiO}_{3}$ single crystal. Journal of Physics-Condensed Matter, 18(8), 2553-2561.

Bogomolov, V. N., Kudinov, E. K., \& Firsov, Yu. A. 1968. Polaron nature of current carriers in rutile. Soviet Physics-Solid State, 9, 2502.

Botton, G. A., Appel, C. C., Horsewell, A., \& Stobbs, W. M. 1995. Quantification of the 
EELS near-edge structures to study Mn doping in oxides. Journal of MicroscopyOxford, 180, 211-216. Part 3.

Brzozowski, E., \& Castro, M. S. 2004. Influence of $\mathrm{Nb}^{5+}$ and $\mathrm{Sb}^{3+}$ dopants on the defect profile, PTCR effect and GBBL characteristics of $\mathrm{BaTiO}_{3}$ ceramics. Journal of the European Ceramic Society, 24(8), 2499-2507.

Butera, A., Fainstein, A., Winkler, E., \& Tallon, J. 2001. Ferromagnetic correlations and mixed $\mathrm{Ru}$ valence in the magnetic superconductor $\mathrm{RuSr} 2(\mathrm{Eu}, \mathrm{Gd}) \mathrm{Cu} 2 \mathrm{O} 8$. Physical Review B, 6305(5). 054442.

Casais, M. T., Alonso, J. A., Rasines, I., \& Hidalgo, M. A. 1995. Preparation, Neutron Structural Study and Characterization of $\mathrm{BaNbO}_{3}$ - a Pauli-Like Metallic Perovskite. Materials Research Bulletin, 30(2), 201-208.

Chakoumakos, B. C., Schlom, D. G., Urbanik, M., \& Luine, J. 1998. Thermal expansion of $\mathrm{LaAlO}_{3}$ and $(\mathrm{La}, \mathrm{Sr})(\mathrm{Al}, \mathrm{Ta}) \mathrm{O}_{3}$, substrate materials for superconducting thin-film device applications. Journal of Applied Physics, 83(4), 1979-1982.

Chan, H. M., Harmer, M. P., \& Smyth, D. M. 1986. Compensating defects in highly donordoped $\mathrm{BaTiO}_{3}$. Journal of the American Ceramic Society, 69(6), 507-510.

Chang, J. Y., Chu, C. F., Huang, C. Y., \& Yueh, R. R. 1999. Optical and photorefractive properties of Nb-doped $\mathrm{BaTiO}_{3}$. Journal of Applied Physics, 85(4), 2318-2322.

Chen, Xin, Wu, Naijuan, Ignatiev, Alex, Zhang, Zuhua, \& Chu, Wei-Kan. 1999. Structure and conducting properties of $\mathrm{La}_{0.5} \mathrm{Sr}_{0.5} \mathrm{CoO}_{3-\delta}$ films on YSZ. Thin Solid Films, 350(1-2), 130-137.

Chrisey, D. B., \& Hubler, G. K. 1994. Pulsed laser deposition of thin films. WileyInterscience.

Coey, J. M. D., Viret, M., \& von Molnar, S. 1999. Mixed-valence manganites. Advances in Physics, 48(2), 167-293.

Cohen, R. E. 1992. Origin of ferroelectricity in perovskite oxides. Nature, 358(6382), 136-138.

Cox, P. A. 1992. Transition metal oxides. an introduction to their electronic structure and properties. Oxford : Clarendon Press ; New York : Oxford University Press. 
Cressey, G., Henderson, C. M. B., \& Vanderlaan, G. 1993. Use of L-Edge X-RayAbsorption Spectroscopy to Characterize Multiple Valence States of 3d TransitionMetals - a New Probe for Mineralogical and Geochemical Research. Physics and Chemistry of Minerals, 20(2), 111-119.

Dagotto, E. 2005. Complexity in strongly correlated electronic systems. Science, 309(5732), 257-262.

Das Sarma, S., \& Hwang, E. H. 2004. Metallicity and its low-temperature behavior in dilute two-dimensional carrier systems. Physical Review B, 69(19).

Das Sarma, S., Lilly, M. P., Hwang, E. H., Pfeiffer, L. N., West, K. W., \& Reno, J. L. 2005. Two-dimensional metal-insulator transition as a percolation transition in a highmobility electron system. Physical Review Letters, 94(13).

de Groot, F. 2005a. Multiplet effects in X-ray spectroscopy. Coordination Chemistry Reviews, 249(1-2), 31-63.

de Groot, F. 2005b. Multiplet effects in X-ray spectroscopy. Coordination Chemistry Reviews, 249(1-2), 31-63.

de Groot, F. M. F., Fuggle, J. C., Thole, B. T., \& Sawatzky, G. A. 1990. L2,3 X-rayabsorption edges of $\mathrm{d}^{0}$ Compounds $-\mathrm{K}^{+}, \mathrm{Ca}^{2+}, \mathrm{Sc}^{3+}$ and $\mathrm{Ti}^{4+}$ in $\mathrm{O}_{h}$ (Octahedral) Symmetry. Physical Review B, 41(2), 928-937.

Desu, S. B., \& Payne, D. A. 1990a. Interfacial segregation in perovskites .3. Microstructure and electrical-properties. Journal of the American Ceramic Society, 73(11), 34073415 .

Desu, S. B., \& Payne, D. A. 1990b. Interfacial segregation in perovskites .4. Internal boundary-layer devices. Journal of the American Ceramic Society, 73(11), 34163421.

Detalle, M., \& Remiens, D. 2008. Chemical and physical characterization of $\mathrm{LaNiO}_{3}$ thin films deposited by sputtering for top and bottom electrodes in ferroelectric structure. Journal of Crystal Growth, 310(15), 3596-3603.

Dijkkamp, D., Venkatesan, T., Wu, X. D., Shaheen, S. A., Jisrawi, N., Minlee, Y. H., McLean, W. L., \& Croft, M. 1987. Preparation of Y-Ba-Cu Oxide Superconductor 
Thin-Films Using Pulsed Laser Evaporation from High-Tc Bulk Material. Applied Physics Letters, 51(8), 619-621.

Economou, E. N., \& Cohen, Morrel H. 1970. Localization in disordered materials: Existence of mobility edges. Physical Review Letters, 25(20), 1445.

Egerton, R. F. 1996. Electron Energy-Loss Spectroscopy in the Electron Microscope. 2nd edition edn. New York: Plenum Press, A division of Plenum Publishing Corporation.

Emin, D. 1996. Pair breaking in semiclassical singlet small-bipolaron hopping. Physical Review B, 53(3), 1260-1268.

Eom, C. B., Vandover, R. B., Phillips, J. M., Werder, D. J., Marshall, J. H., Chen, C. H., Cava, R. J., Fleming, R. M., \& Fork, D. K. 1993. Fabrication and Properties of Epitaxial Ferroelectric Heterostructures with $\mathrm{SrRuO}_{3}$ Isotropic Metallic Oxide Electrodes. Applied Physics Letters, 63(18), 2570-2572.

Feltz, A., \& Langbein, H. 1977. Investigations on electronically conducting oxide systems .10. Electrical-conductivity and optical-absorption of a-domain and c-domain crystals of $\mathrm{BaNb}_{x}^{V} \mathrm{Ti}_{x}^{I I I} \mathrm{Ti}_{1-2 x}^{I V} \mathrm{O}_{3}$. Ferroelectrics, 15(1-2), 7-14.

Frohlich, H. 1954. Electrons in lattice fields. Advances in Physics, 3(11), 325-361.

GADDS4.0. 1999. General area detector diffraction system (GADDS) user's manual, Bruker Analytical X-ray Systems. Version 4.0 edn.

Gasparov, V. A., Strukova, G. K., \& Khassanov, S. S. 1994. Superconductivity above 20-K in Barium-Niobium-Oxide Compounds. Jetp Letters, 60(6), 440-444.

Gasparov, V. A., Ermolov, S. N., Strukova, G. K., Sidorov, N. S., Khassanov, S. S., Wang, H. S., Schneider, M., Glaser, E., \& Richter, W. 2001. Superconducting and anomalous electron transport properties and electronic structure of $\mathrm{BaNbO}_{3-x}$ and $\mathrm{Ba}_{2} \mathrm{Nb}_{5} \mathrm{O}_{x}$ films. Physical Review B, 6317(17).

Gatan, Inc. 2000. EELS imageing and analysis school.

Gilbert, S. R., Wills, L. A., Wessels, B. W., Schindler, J. L., Thomas, J. A., \& Kannewurf, C. R. 1996. Electrical transport properties of epitaxial $\mathrm{BaTiO}_{3}$ thin films. Journal of Applied Physics, 80(2), 969-977. 
Graetz, J., Ahn, C. C., Ouyang, H., Rez, P., \& Fultz, B. 2004. White lines and d-band occupancy for the $3 d$ transition-metal oxides and lithium transition-metal oxides. Physical Review B, 69(23). 235103.

Guo, H. Z., Liu, L. F., Ding, S., Lu, H. B., Zhou, Y. L., Cheng, B. L., \& Chen, Z. H. 2004. Structural phase transitions of $\mathrm{BaNb}_{x} \mathrm{Ti}_{1-x} \mathrm{O}_{3}\left(0_{i} 0_{i}=\mathrm{x}_{i}=0.5\right)$ thin films. Journal of Applied Physics, 96(6), 3404-3407.

Guo, X. G., Chen, X. S., \& Lu, W. 2003. Optical properties of $\mathrm{Nb}$ doped $\mathrm{SrTiO}_{3}$ from first principles study. Solid State Communications, 126(8), 441-446.

Harada, J., Pederson, T., \& Barnea, Z. 1970. X-ray and neutron diffraction study of tetragonal barium titanate. Acta Crystallography A, 26, 336-344.

Hentzell, H. T. G., Grovenor, C. R. M., \& Smith, D. A. 1984. Grain-Structure Variation with Temperature for Evaporated Metal-Films. Journal of Vacuum Science \& Technology a-Vacuum Surfaces and Films, 2(2), 218-219.

Holstein, T. 1959. Studies of polaron motion Part II. The "small" polaron. Annals of Physics, 8(3), 343-389.

Huang, Y. H., Zhao, K., Lu, H. B., Jin, K. J., He, M., Chen, Z. H., Zhou, Y. L., \& Yang, G. Z. 2006. Multifunctional characteristics of $\mathrm{BaNb}_{0.3} \mathrm{Ti}_{0.7} \mathrm{O}_{3} / \mathrm{Si}$ p-n junctions. Applied Physics Letters, 88(6).

Huijben, M., Rijnders, G., Blank, D. H. A., Bals, S., Van Aert, S., Verbeeck, J., Van Tendeloo, G., Brinkman, A., \& Hilgenkamp, H. 2006. Electronically coupled complementary interfaces between perovskite band insulators. Nature Materials, 5(7), 556-560.

Inoue, I. H., Hase, I., Aiura, Y., Fujimori, A., Haruyama, Y., Maruyama, T., \& Nishihara, Y. 1995. Systematic development of the spectral-function in the $3 \mathrm{~d}^{1}$ Mott-Hubbard System $\mathrm{Ca}_{1-x} \mathrm{Sr}_{x} \mathrm{VO}_{3}$. Physical Review Letters, 74(13), 2539-2542.

Inoue, I. H., Bergemann, C., Bergemann, I., \& Julian, S. R. 2002. Fermi Surface of $3 \mathrm{~d}^{1}$ Perovskite $\mathrm{CaVO}_{3}$ near the Mott Transition. Physical Review Letters, 88(23).

Jones, R. O., \& Gunnarsson, O. 1989. The density functional formalism, its applications and prospects. Reviews of Modern Physics, 61, 689.

Jonker, G. H., \& Havinga, E. E. 1982. The influence of foreign Ions on the crystal-lattice 
of barium-titanate. Materials Research Bulletin, 17(3), 345-350. Times Cited: 80.

Kan, D. S., Terashima, T., Kanda, R., Masuno, A., Tanaka, K., Chu, S. C., Kan, H., Ishizumi, A., Kanemitsu, Y., Shimakawa, Y., \& Takano, M. 2005. Blue-light emission at room temperature from $\mathrm{Ar}^{+}$-irradiated $\mathrm{SrTiO}_{3}$. Nature Materials, 4(11), 816-819.

Keast, V. J., Scott, A. J., Brydson, R., Williams, D. B., \& Bruley, J. 2001. Electron energyloss near-edge structure - a tool for the investigation of electronic structure on the nanometre scale. Journal of Microscopy-Oxford, 203, 135-175.

Khan, M. N., Kim, H. T., Kusawake, T., Kudo, H., Ohshima, K., \& Uwe, H. 1999. X-ray diffraction and Rutherford backscattering spectrometry of $\mathrm{BaNb}_{x} \mathrm{Ti}_{1-x} \mathrm{O}_{3}$ thin films synthesized by laser ablation. Journal of Applied Physics, 86(4), 2307-2310.

Kokalj, A. 2003. Computer graphics and graphical user interfaces as tools in simulations of matter at the atomic scale. Computational Materials Science, 28(155-168).

Kolodiazhnyi, T. 2008. Insulator-metal transition and anomalous sign reversal of the dominant charge carriers in perovskite $\mathrm{BaTiO}_{3-\delta}$. Physical Review $B, 78(045107)$.

Kolodiazhnyi, T., \& Wimbush, S. C. 2006. Spin-singlet small bipolarons in Nb-doped $\mathrm{BaTiO}_{3}$. Physical Review Letters, 96(24).

Koonce, C. S., Cohen, Marvin L., Schooley, J. F., Hosler, W. R., \& Pfeiffer, E. R. 1967. Superconducting transition temperatures of semiconducting $\mathrm{SrTiO}_{3}$. Physical Review, 163(2), 380 .

Koschek, G., \& Kubalek, E. 1985. Grain-boundary characteristics and their influence on the electrical resistance of barium-titanate ceramics. Journal of the American Ceramic Society, 68(11), 582-586.

Kramer, B., \& Mackinnon, A. 1993. Localization - Theory and Experiment. Reports on Progress in Physics, 56(12), 1469-1564.

Kurata, H., Lefevre, E., Colliex, C., \& Brydson, R. 1993. Electron-energy-loss near-edge structures in the oxygen K-edge spectra of transition-metal oxides. Physical Review $B, 47(20), 13763$.

Kutty, T. R. N., Murugaraj, P., \& Gajbhiye, N. S. 1985. Electron-paramagnetic-res evidence for activation of trap centers in PTCR $\mathrm{BaTiO}_{3}$ ceramics. Materials Research Bulletin, 
$20(5), 565-574$.

Lang, I. G., \& Firsov, Y. A. 1963. Kinetic theory of semiconductors with low mobility. Soviet Physics JETP, 16(5), 1301-1312.

Lee, J. J., Thio, C. L., \& Desu, S.B. 1995. Electrode contacts on ferroelectric $\mathrm{Pb}\left(\mathrm{Zr}_{x} \mathrm{Ti}_{1-x}\right) \mathrm{O}_{3}$ and $\mathrm{SrBi}_{2} \mathrm{Ta}_{2} \mathrm{O}_{9}$ thin films and their influence on fatigue properties. Journal of Applied Physics, 78(8), 5073-5078.

Lee, J. S., Lee, Y. S., Noh, T. W., Oh, S. J., Yu, J. J., Nakatsuji, S., Fukazawa, H., \& Maeno, Y. 2002. Electron and orbital correlations in $\mathrm{Ca}_{2-x} \mathrm{Sr}_{x} \mathrm{RuO}_{4}$ probed by optical spectroscopy. Physical Review Letters, 89(25).

Lemée, N., Dubourdieu, C., Delabouglise, G., Sénateur, J. P., \& Laroudie, F. 2002. Semiconductive $\mathrm{Nb}$-doped $\mathrm{BaTiO}_{3}$ films grown by pulsed injection metalorganic chemical vapor deposition. Journal of Crystal Growth, 235(1-4), 347-351.

Lenjer, S., Schirmer, O. F., Hesse, H., \& Kool, T. W. 2002. Conduction states in oxide perovskites: Three manifestations of $\mathrm{Ti}^{3+} \mathrm{Jahn}$-Teller polarons in barium titanate. Physical Review B, 66(16).

Levis, G.V., \& Catlow, C. R. A. 1986. Defect studies of doped and undoped barium titanate using computer simulation techniques. Journal of Physics and Chemistry of Solids, 47(1), 89-97.

Liou, J. W., \& Chiou, B. S. 1997. Dielectric characteristics of doped $\mathrm{Ba}_{1-x} \mathrm{Sr}_{x} \mathrm{TiO}_{3}$ at the paraelectric state. Materials Chemistry and Physics, 51(1), 59-63.

Liu, L. F., Guo, H. Z., Lu, H. B., Dai, S. Y., Cheng, B. L., \& Chen, Z. H. 2005. Effects of donor concentration on the electrical properties of $\mathrm{Nb}$-doped $\mathrm{BaTiO}_{3}$ thin films. Journal of Applied Physics, 97(5).

Liu, Y. X., Masumoto, H., \& Goto, T. 2004. Electrical and optical properties of $\mathrm{IrO}_{2}$ thin films prepared by laser-ablation. Materials Transactions, 45(10), 3023-3027.

Marco, J. F., Gancedo, J. R., \& Berry, F. J. 1997. The oxidation states of titanium and niobium in compounds of composition $\mathrm{Sn}_{x} \mathrm{NbTiP}_{3} \mathrm{O}_{1} 2\left(0_{j} \mathrm{x} \leq 0.50\right)$ : An XPS study. Polyhedron, 16(17), 2957-2961.

Marucco, J. F., Ocio, M., Forget, A., \& Colson, D. 1997. Synthesis and electrical properties 
of solid solutions $\mathrm{BaNb}_{1-x} \mathrm{Ti}_{x} \mathrm{O}_{3}$ with $0_{i} \mathrm{x}_{i} 0.8$. Journal of Alloys and Compounds, 262, 454-458.

Maso, N., Beltran, H., Cordoncillo, E., Flores, A. A., Escribano, P., Sinclair, D. C., \& West, A. R. 2006. Synthesis and electrical properties of Nb-doped $\mathrm{BaTiO}_{3}$. Journal of Materials Chemistry, 16(30), 3114-3119.

Mattheiss, L. F. 1969. Band structure and Fermi surface of $\mathrm{ReO}_{3}$. The Physical Review, 181(3).

Maunders, C., Martin, B. E., Wei, P., Petric, A., \& Botton, G. A. 2008. Investigation of the electronic structure of the cubic spinel Cu1.2Mn1.8O4 using electron energy loss spectroscopy. Solid State Ionics, 179(19-20), 718-724.

Miki, T., Fujimoto, A., \& Jida, S. 1998. An evidence of trap activation for positive temperature coefficient of resistivity in $\mathrm{BaTiO}_{3}$ ceramics with substitutional $\mathrm{Nb}$ and $\mathrm{Mn}$ as impurities. Journal of Applied Physics, 83(3), 1592-1603.

Mitchell, Roger H. 2002. Perovskites: mondern and ancient. Thunder Bay, Ontario. : Almaz Press.

Mott, N. F. 1974. Metal-insulator transitions. London : Taylor \& Francis ; New York : Barnes \& Noble Books,.

Mott, N. F., \& Davis, E. A. 1979. Electronic processes in non-crystalline materials. 2d ed. edn. Oxford : Clarendon Press.

Nagano, D., Funakubo, H., Shinozaki, K., \& Mizutani, N. 1998. Electrical properties of semiconductive $\mathrm{Nb}$-doped $\mathrm{BaTiO}_{3}$ thin films prepared by metal-organic chemicalvapor deposition. Applied Physics Letters, 72(16), 2017-2019.

Ohring, Milton. 2002. Materials Science of Thin Films: deposition and structure. 2nd edition edn. San Diego, CA : Academic Press.

Orgzall, I., Lorenz, B., Ting, S. T., Hor, P. H., Menon, V., Martin, Ch R., \& Hochheimer, H. D. 1996. Thermopower and high-pressure electrical conductivity measurements of template synthesized polypyrrole. Physical Review B, 54(23), 16654.

Paterson, J. H., \& Krivanek, O. L. 1990. Elnes of $3 d$ Transition-Metal Oxides .2. Variations with oxidation-state and crystal-structure. Ultramicroscopy, 32(4), 319-325. 
PhD Thesis—Yang Shao-McMaster University-Materials Science and Engineering 2009

Paz de Araujo, C., Scott, J. F., \& Taylor, G. W. 1996. Ferroelectric thin films: synthesis and basic properties. Taylor \& Francis.

Pearson, D. H., Ahn, C. C., \& Fultz, B. 1993. White lines and d-electron occupancies for the $3 d$ and $4 d$ transition-metals. Physical Review B, 47(14), 8471-8478.

Pekar, S. 1946. The method of effective electron mass in crystals. Zh. Eksp. Teor. Fiz., 16, 335.

Peng, C. J., \& Lu, H. Y. 1988. Compensation effect in semiconducting barium-titanate. Journal of the American Ceramic Society, 71(1), C44-C46.

Pfeiffer, E. R., \& Schooley, J. F. 1969. Superconducting transition temperatures of Nbdoped $\mathrm{SrTiO}_{3}$. Physics Letters A, 29(10), 589-590.

Poulsen, H. F., Andersen, N. H., Andersen, J. V., Bohr, H., \& Mouritsen, O. G. 1991. Relation between superconducting transition-temperature and oxygen ordering in $\mathrm{YBa}_{2} \mathrm{Cu}_{3} \mathrm{O}_{6+x}$. Nature, 349(6310), 594-596.

Ramesh, R., Chan, W. K., Wilkens, B., Gilchrist, H., Sands, T., Tarascon, J. M., \& Keramidas, V. G. 1992. Fatigue and retention in ferroelectric Y-Ba-Cu-O/Pb-Zr-Ti-O/Y-BaCu-O heterostructures. Applied Physics Letters, 61, 1537.

Rao, C. N. R., \& Raveau, B. 1995. Transition metal oxides. New York: VCH.

Rask, J. H., Miner, B. A., \& Buseck, P. R. 1987. Determination of manganese oxidationstates in solids by electron energy-loss spectroscopy. Ultramicroscopy, 21(4), 321326.

Sarma, D. D., Chainani, A., Krishnakumar, S. R., Vescovo, E., Carbone, C., Eberhardt, W., Rader, O., Jung, C., Hellwig, C., Gudat, W., Srikanth, H., \& Raychaudhuri, A. K. 1998. Disorder effects in electronic structure of substituted transition metal compounds. Physical Review Letters, 80(18), 4004-4007.

Sayer, M., \& Sreenivas, K. 1990. Ceramic Thin-Films - Fabrication and Applications. Science, 247(4946), 1056-1060.

Schlom, D. G., Chen, L. Q., Eom, C. B., Rabe, K. M., Streiffer, S. K., \& Triscone, J. M. 2007. Strain tuning of ferroelectric thin films. Annual Review of Materials Research, $37,589-626$. 
Schofield, P. F., Henderson, C. M. B., Cressey, G., \& Vanderlaan, G. 1995. 2p X-rayabsorption spectroscopy in the earth-sciences. Journal of Synchrotron Radiation, 2, 93-98.

Schooley, J. F., Hosler, W. R., \& Cohen, Marvin L. 1964. Superconductivity in semiconducting $\mathrm{SrTiO}_{3}$. Physical Review Letters, 12(17), 474.

Shao, Y., Hughes, R. A., Dabkowski, A., Radtke, G., Gong, W. H., Preston, J. S., \& Botton, G. A. 2008. Structural and transport properties of epitaxial niobium-doped $\mathrm{BaTiO}_{3}$ films. Applied Physics Letters, 93, 192114.

Shklovskii, B. I., \& Efros, A. L. 1984. Electronic properties of doped semiconductors. Berlin ; New York : Springer-Verlag.

Span, E. A. F., Roesthuis, F. J. G., Blank, D. H. A., \& Rogalla, H. 1999. Structural and electrical properties of $\mathrm{La}_{0.5} \mathrm{Sr}_{0.5} \mathrm{CoO}_{3}$ films on $\mathrm{SrTiO}_{3}$ and porous alpha- $\mathrm{Al}_{2} \mathrm{O}_{3}$ substrates. Applied Physics a-Materials Science \& Processing, 69, S783-S785.

Spence, J. C. H. 2003. Oxygen in crystals - Seeing is believing. Science, 299(5608), 839841.

Strukova, G. K., Kedrov, V. V., Zverev, V. N., Khasanov, S. S., Ovchinnikov, I. M., Batov, I. E., \& Gasparov, V. A. 1997. On the synthesis and the electric and magnetic properties of superconducting barium-niobium-oxide compounds. Physica C, 291(3-4), 207-212.

Ting, C. J., Peng, C. J., Lu, H. Y., \& Wu, S. T. 1990. Lanthanum-magnesium and lanthanum-manganese donor-acceptor-codoped semiconducting barium titanate. Journal of the American Ceramic Society, 73(2), 329-334.

Tomio, T., Miki, H., Tabata, H., Kawai, T., \& Kawai, S. 1994. Control of electricalconductivity in laser-deposited $\mathrm{SrTiO}_{3}$ thin-films with $\mathrm{Nb}$ doping. Journal of Applied Physics, 76(10), 5886-5890.

Torrance, J. B., Lacorre, P., Asavaroengchai, C., \& Metzger, R. M. 1991. Why are some oxides metallic, while most are insulating. Physica $C$, 182(4-6), 351-364.

van der Pauw, L. J. 1958. A method of measuring the resistivity and hall coefficient of lamellae of arbitrary shape. Philips Technical Reivew, 20, 220-224. 
Varma, C. M. 1976. Mixed-Valence Compounds. Reviews of Modern Physics, 48(2), 219238.

Wernicke, R. 1978. Influence of kinetic processes on electrical-conductivity of donor-doped $\mathrm{BaTiO}_{3}$ ceramics. Physica Status Solidi a-Applied Research, 47(1), 139-144.

Wu, T. B., \& Lin, J. N. 1994. Transition of Compensating Defect Mode in Niobium-Doped Barium-Titanate. Journal of the American Ceramic Society, 77(3), 759-764.

Wu, X. D., Dijkkamp, D., Ogale, S. B., Inam, A., Chase, E. W., Miceli, P. F., Chang, C. C., Tarascon, J. M., \& Venkatesan, T. 1987. Epitaxial ordering of oxide superconductor thin-films on (100) $\mathrm{SrTiO}_{3}$ prepared by pulsed laser evaporation. Applied Physics Letters, 51(11), 861-863.

Yoon, S. H., \& Kim, H. 2002. Effect of donor (Nb) concentration on the bulk electrical resistivity of Nb-doped barium titanate. Journal of Applied Physics, 92(2), 10391047.

Yoon, S. H., Lee, K. H., \& Kim, H. 2000. Effect of acceptors on the segregation of donors in niobium-doped barium titanate positive temperature coefficient resistors. Journal of the American Ceramic Society, 83(10), 2463-2472.

Zaanen, J., \& Sawatzky, G. A. 1990. Systematics in band-gaps and optical-spectra of 3d transition-metal Compounds. Journal of Solid State Chemistry, 88(1), 8-27.

Zaanen, J., Sawatzky, G. A., \& Allen, J. W. 1985a. Band-gaps and electronic-structure of transition-metal compounds. Physical Review Letters, 55(4), 418-421.

Zaanen, J., Sawatzky, G. A., Fink, J., Speier, W., \& Fuggle, J. C. 1985b. L2,3 AbsorptionSpectra of the Lighter $3 d$ Transition-Metals. Physical Review B, 32(8), 4905-4913.

Zhao, G. M., Smolyaninova, V., Prellier, W., \& Keller, H. 2000. Electrical transport in the ferromagnetic state of manganites: Small-polaron metallic conduction at low temperatures. Physical Review Letters, 84(26), 6086-6089. 